\title{
Copyright
}

by

Abdulwahab Abdullatif Bukhari

2011 
The Thesis Committee for Abdulwahab Abdullatif Bukhari Certifies that this is the approved version of the following thesis:

\section{Optimization of Production Allocation under Price Uncertainty: Relating Price Model Assumptions to Decisions}

\section{APPROVED BY \\ SUPERVISING COMMITTEE:}

Supervisor:

Christopher J. Jablonowski

Leon S. Lasdon

James S. Dyer 


\title{
Optimization of Production Allocation under Price Uncertainty: Relating Price Model Assumptions to Decisions
}

by

Abdulwahab Abdullatif Bukhari, B.S.

\author{
Thesis \\ Presented to the Faculty of the Graduate School of \\ The University of Texas at Austin \\ in Partial Fulfillment \\ of the Requirements \\ for the Degree of
}

Master of Science in Engineering

The University of Texas at Austin

August 2011 


\section{Dedication}

To my (sweetheart) daughter Lamar, parents, and wife. 


\section{Acknowledgements}

I am very thankful to Saudi ARAMCO for their financial support. That support gave me the opportunity to pursue my graduate studies in one of the top universities in Petroleum Engineering, The University of Texas at Austin, perform research and increase my knowledge of petroleum economic and decision and risk analysis in preparation for my upcoming challenges in the oil industry.

I would like to offer my sincere thanks to my advisor, Dr. Christopher Jablonowski, for his support and guidance over the last two years. His support in difficult times, and his knowledge and encouragement resulted in the final product of this study.

I am deeply grateful to my mentor at Saudi ARAMCO, Knut Olsen, for his unconditional support and guidance. His motivation and advice encouraged me to aim for the best university in petroleum engineering. Knut has been a great teacher for me over the last three years.

Special gratitude is also extended to Dr. Marco Dias, Adjunct Professor in the Industrial Engineering department at the PUC-RIO and Senior Petroleum Engineer at Petrobras, for his expert guidance and help in the building of the stochastic price forecast models. I would like to offer my sincere thanks to Dr. Leon Lasdon, professor at the McCombs School of Business, for offering courses on optimization theories and decision support modeling, and for his advice and recommendations during my study. Many thanks are also due to Dr. Ghislaine De Regge, professor in the Civil Engineering department, for her time and effort reviewing and correcting the thesis. My heartfelt 
appreciation also goes out to Dr. Jim Dyer, professor at the McCombs School of Business, for his advice and recommendations.

Finally, I would like to acknowledge the support and prayers of my parents, Abdullatif and Saadiah; my wife, Alaa; and my brother and sisters, Mousa, Riham, Saja, and Maryam. I would like to thank my beloved daughter Lamar for growing the happiness inside my heart.

June 23, 2011 


\title{
Abstract \\ Optimization of Production Allocation under Price Uncertainty: Relating Price Model Assumptions to Decisions
}

\author{
Abdulwahab Abdullatif Bukhari, M.S.E. \\ The University of Texas at Austin, 2011 \\ Supervisor: Christopher J. Jablonowski
}

\begin{abstract}
Allocating production volumes across a portfolio of producing assets is a complex optimization problem. Each producing asset possesses different technical attributes (e.g. crude type), facility constraints, and costs. In addition, there are corporate objectives and constraints (e.g. contract delivery requirements). While complex, such a problem can be specified and solved using conventional deterministic optimization methods. However, there is often uncertainty in many of the inputs, and in these cases the appropriate approach is neither obvious nor straightforward. One of the major uncertainties in the oil and gas industry is the commodity price assumption(s). This paper investigates this problem in three major sections: (1) We specify an integrated stochastic optimization model that solves for the optimal production allocation for a portfolio of producing assets when there is uncertainty in commodity prices, (2) We then compare the solutions that vii
\end{abstract}


result when different price models are used, and (3) We perform a value of information analysis to estimate the value of more accurate price models. The results show that the optimum production allocation is a function of the price model assumptions. However, the differences between models are minor, and thus the value of choosing the "correct" price model, or similarly of estimating a more accurate model, is small. This work falls in the emerging research area of decision-oriented assessments of information value. 


\section{Table of Contents}

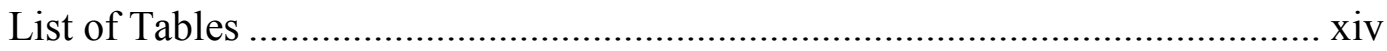

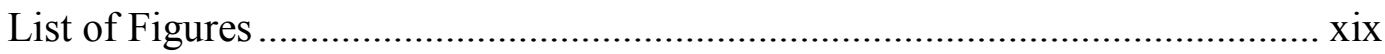

CHAPTER 1: INTRODUCTION 1

Chapter 2: Crude ANd Pricing General Information 4

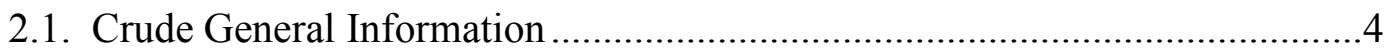

2.2. Estimating the Properties of Crude Price Distribution .....................................

2.2.1. Mean Price and Standard Deviation Estimation ................................... 8

2.2.1.1. Estimation of Mean Price..........................................................9

2.2.1.2. Estimation of Standard Deviation...........................................10

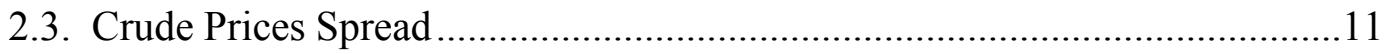

CHAPTER 3: RISK ANALYSIS WORKFLOW

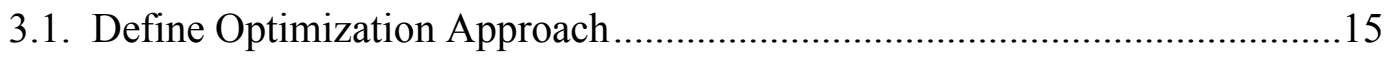

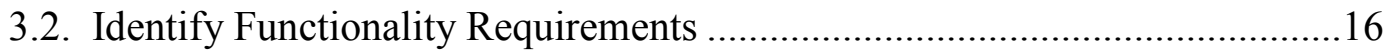

3.3. Select Price Forecasting Approach ………………...................................... 16

3.4. Initiate Production Rate Allocation ..................................................................17

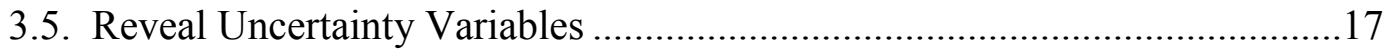

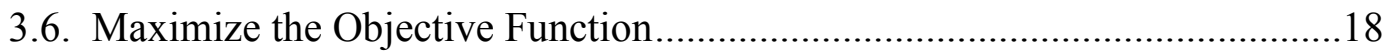

3.7. Generate Probability Density Function for the Objective Function ...............19

3.8. Solve for the Optimum Production Allocation .................................................19

Chapter 4: Price Models

4.1. Collecting Historical Price Data .....................................................................23

4.2. Programming Price Forecasting Models.....................................................24

4.2.1. Crude Oil Price Modeling Parameters .................................................24 
4.2.1.1. Crude Mean Price ....................................................................25

4.2.1.2. The Rate of Crude Oil Price increases ......................................27

4.2.1.3. The Growth Rate of the Standard Deviation............................27

4.2.1.4. Crude Oil Price Volatility $(\sigma)$.................................................28

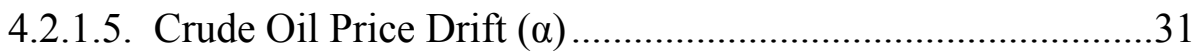

4.2.1.6. Long-run Equilibrium Price $\left(\mathrm{P}^{*}\right)$ and Price Reversion Rate $(\eta) 32$

4.2.1.7. Jump Size $(\phi)$ and Frequency $(\lambda)$..........................................34

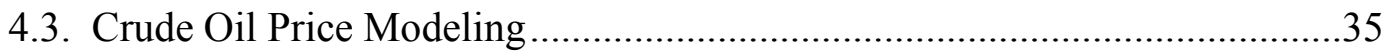

4.3.1. Deterministic Forecasting Models .......................................................35

4.3.1.1. The Flat Price Model ..............................................................35

4.3.1.2. The Floating Price Model ......................................................36

4.3.2. Probabilistic Forecasting Models......................................................38

4.3.2.1. The Independent Lognormal Distribution (ILD) ....................38

4.3.2.2. The Geometric Brownian Motion (GBM) ..............................41

4.3.2.2.1. The GBM Model, Background ....................................41

4.3.2.2.2. The GBM Formulation ................................................44

4.3.2.3. Mean Reversion Model (MRM) ............................................46

4.3.2.3.1. Mean Reversion Model, Background ..........................46

4.3.2.3.2. Mean Reversion Model Formulation ...........................47

4.3.2.4. Mean Reversion with Jumps Model (MRJM) .........................49

4.3.2.4.1. Mean Reversion with Jumps Model, Background .....49

4.3.2.4.2. Mean Reversion with Jumps Model Formulation......52

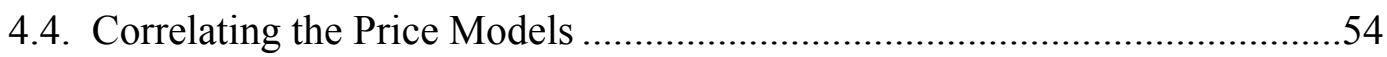

CHAPTER 5: INTEgRATED OPTIMIZATION MODEL 60

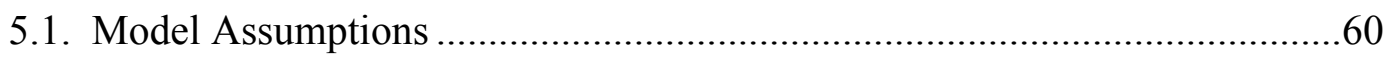

5.1.1. Probabilistic Crude Oil Price Modeling Assumptions .........................60

5.1.2. Field Parameter Estimation Assumptions ............................................60

5.1.3. General Modeling Assumptions ..........................................................61 
5.2. Integrated Model Flowchart. 62

5.2.1. Integrated Model Flowchart Description (Optimization Process) .....64 5.2.1.1. Choosing Crude Price Forecasting Process (User Involved) 64 5.2.1.2. Sampling Crude Oil Price Process ...........................................65

5.2.1.3. Input Parameters and Data ...................................................66

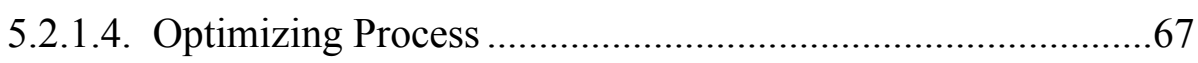

5.3. Optimization Problem Formulation .............................................................68

5.3.1. Optimization Problem Formulation for the Deterministic Price Forecast

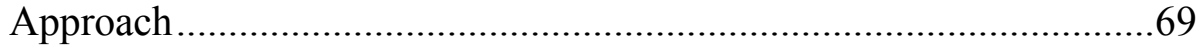

5.3.1.1. Definition of Modeling Parameters .........................................70

5.3.1.2. Modeling Decision Variables ................................................71

5.3.1.3. Optimization Model Calculations ..............................................71

5.3.1.4. Optimization Problem Formulation .........................................72

5.3.2. Optimization Problem Formulation for the Probabilistic Price Forecast Approach .....................................................................................73

5.3.2.1. Definition of Modeling Parameters .........................................74

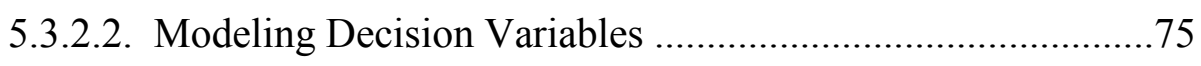

5.3.2.3. Optimization Model Calculations ………………………........76

5.3.2.4. Optimization Problem Formulation .........................................76

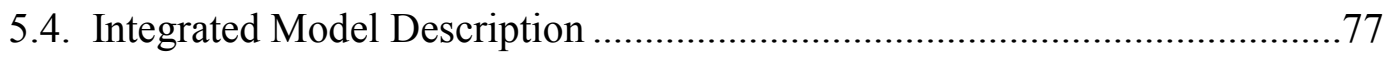

5.4.1. Integrated Model Modules .............................................................78

5.4.1.1. Integrated Model Parameters Module ………………….........78

5.4.1.2. Cash Flow Calculations Module ............................................. 83

5.4.1.2.1. Crude Oil Price Forecasting Sub-Module...................83

5.4.1.2.2. Oil Production Sub-Module.........................................83

5.4.1.2.3. Deductions Sub-Module ..............................................84

5.4.1.2.4. Tax Payments Sub-Module...........................................85

5.4.1.2.5. Non-Tax Items Sub-Module ……………………..........85

5.4.1.2.6. Net Cash Flow and NPV Calculations Sub-Module...85

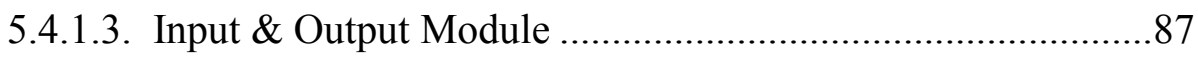


5.4.1.3.1. Users' Input Sub-Module..............................................87

5.4.1.3.2. Objective Function Sub-Module..................................91

5.4.1.3.3. Decision Variables Sub-Module ...................................92

5.4.1.3.4. Constraints Sub-Module ...............................................93

ChAPTER 6: RESUltS AND Discussion 94

6.1. The Deterministic Price Models ...................................................................94

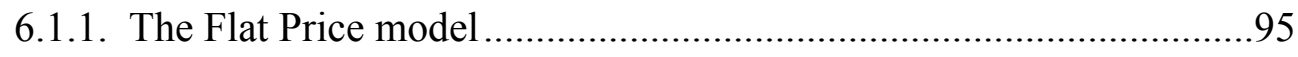

6.1.1.1. The Optimum Decision of the Flat Price Model.....................96

6.1.1.2. The Sensitivity Analysis of the Flat Price Model ...................99

6.1.2. The Floating Price Model ................................................................107

6.1.2.1. The Optimum Decision of the Floating Price Model...........108

6.1.2.2. The Sensitivity Analysis of the Floating Price Model ..........111

6.2. The Probabilistic Price Models …………………....................................118

6.2.1. The Independent Log-normal Distribution Price Model (ILD) .......118

6.2.1.1. The Optimum Decision of the ILD Forecast Model .............119

6.2.1.2. The Sensitivity Analysis of the ILD Forecast Model ..........122

6.2.2. The Geometric Brownian Motion (GBM) .....................................128

6.2.2.1. The Optimum Decision of the Geometric Brownian Motion

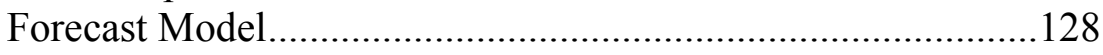

6.2.2.2. The Sensitivity Analysis of the Geometric Brownian Motion (GBM) (.........................................................................130

6.2.3. The Mean Reversion Model (MRM) ..............................................134

6.2.3.1. The Optimum Decision of the Mean Reversion Model (MRM)

6.2.3.2. The Sensitivity Analysis of the Mean Reversion Model (MRM) .137

6.2.4. The Mean Reversion with Jumps Model (MRJM) ...........................140

6.2.4.1. The Optimum Decision of the Mean Reversion with Jumps

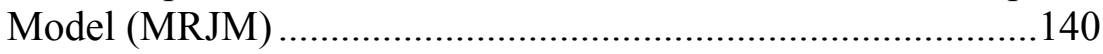

6.2.4.2. The Sensitivity Analysis of the Mean Reversion with Jumps Model (MRJM) ......................................................................142 


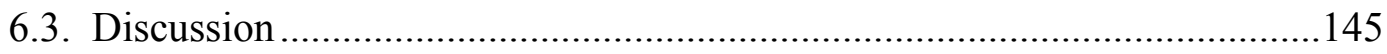

Chapter 7: The Value of an Accurate Price Correlation Model (VOI ANALYSIS) 150

7.1. The VOI Analysis on Heavy-Medium Price Correlation Model ..................150

7.2. The VOI Analysis on Heavy-Light Price Correlation Model .......................154

7.3. The VOI Analysis on Heavy-XLight Price Correlation Model ....................157

7.4. The VOI Analysis on Heavy-XXLight Price Correlation Model .................159

CHAPTER 8: CONCLUSIONS AND RECOMMENDATIONS FOR FUTURE WORK 162

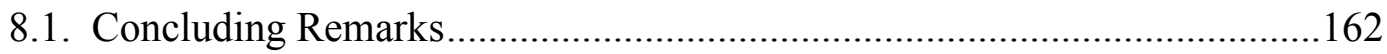

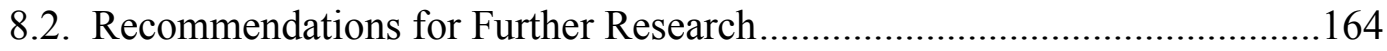

8.2.1. Recommendations Related to Price Correlation .................................164

8.2.2. Recommendations Related to Optimization Process ..........................165

8.2.3. Recommendations Related to Technical Process .............................166

$\begin{array}{ll}\text { REFERENCES } & 167\end{array}$

VITA 169 


\section{List of Tables}

Table 2.1: Crude Grade Classification ................................................................

Table 2.2: Crudes Probability Distribution Properties ...........................................

Table 2.3: Properties of the Probability Distributions of the Available Crude Oil

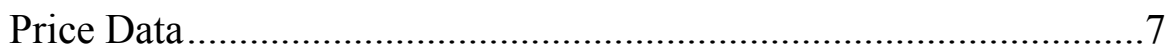

Table 2.4: Crude Mean Price and Standard Deviation Calculations for the Missing Crudes 11

Table 2.5: The Properties of the Probability Distribution for the Crude Oil Prices

Table 2.6: The Properties of Probability Distribution for the Spread of the Crude

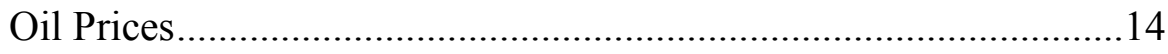

Table 4.1: The Classifications of the Crudes .....................................................23

Table 4.2: Probability Distribution Properties of the Crudes...............................24

Table 4.3: Annual Crude Prices (2004-2010) ……………………………….....26

Table 4.4: The Light Crude Volatility Calculations Using Annual Historical Data 30

Table 4.5: Volatility Values for Different Stochastic Processes ..........................31

Table 4.6: The Light Crude Drift Calculations Using Annual Historical Data .31

Table 4.7: The Heavy Crude Regression Analysis for $\mathrm{P}^{*}$ and $\eta$ Estimation ......33

Table 4.8: Regression Analysis (AR(1)) Parameters ...........................................34

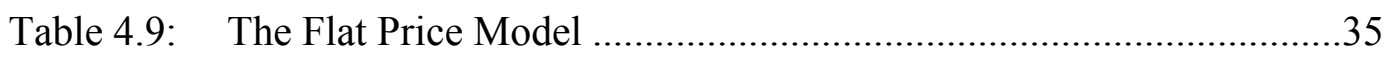

Table 4.10: Initial Crude Prices and Annual Growth Rates Used in the Floating Price

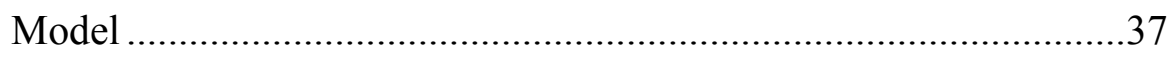

Table 4.11: The Floating Price Model ............................................................... 
Table 4.12: Crude Mean Prices and Initial Standard Deviations

Table 4.13: The Growth Rate of the Heavy Price Standard Deviation, and the Heavy Crude Minimum Price 40

Table 4.14: The Heavy Crude Standard Deviation ............................................40

Table 4.15: The ILD Price Forecast Model ...................................................40

Table 4.16: The Heavy Crude GBM Modeling Parameters................................44

Table 4.17: Standard Normal Distribution........................................................45

Table 4.18: The GBM Forecasting Model ......................................................45

Table 4.19: The GBM Correlated Price Forecasts for the Medium, Light, X-Light,

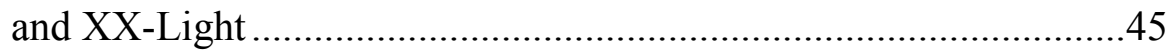

Table 4.20: The Heavy Crude MRM Forecasting Parameters .............................48

Table 4.21: Standard Normal Distribution......................................................48

Table 4.22: The Heavy Crude Mean Reversion Model ......................................49

Table 4.23: Correlated Mean Reversion Models for the Medium, Light, X-Light, and XX-Light Crudes ...............................................................49

Table 4.24: Mean Reversion with Jumps Modeling Weekly Parameters ............53

Table 4.25: Jumps Parameters......................................................................5

Table 4.26: Poisson Process ...........................................................................54

Table 4.27: Mean Reversion with Jumps Model Forecasts ...............................54

Table 4.28: Annual Forecasted Crude Prices Using Mean Reversion with Jumps54

Table 4.29: Crude Price Correlation Factors....................................................55

Table 4.30: Annual Historical Prices for the Light, Medium and Heavy Crudes 57

Table 4.31: Crude Price Correlation Parameters.................................................58

Table 4.32: Standard Deviation of the Residual ..............................................59

Table 4.33: Regression Residual by Crude Grades..........................................59 
Table 5.1: Field Parameter Estimation Assumptions......................................61

Table 5.2: Basic Parameters of the Fields .....................................................67

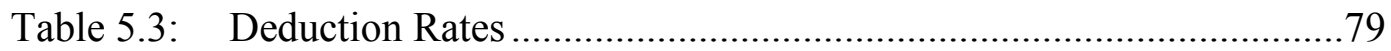

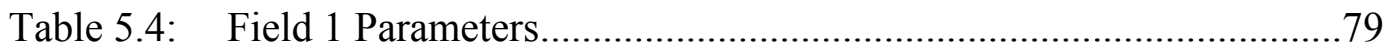

Table 5.5: Field Parameter Assumptions ...................................................... 82

Table 5.6: Field Parameters......................................................................... 82

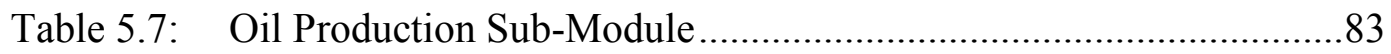

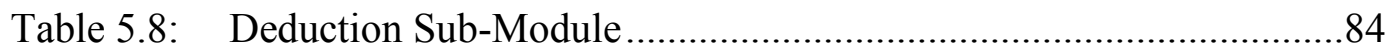

Table 5.9: Input Sub-Module for the Flat Price Forecasting Model .................87

Table 5.10: Input Sub-Module for the Floating Price Forecasting Model ...........88

Table 5.11: Input Sub-Module for the Independent Log-normal Distribution Price Forecasting Model .............................................................. 88

Table 5.12: Input Sub-Module for the Geometric Brownian Motion Price

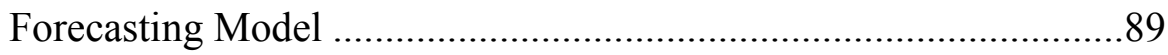

Table 5.13: Input Sub-Module for the Mean Reversion Price Forecasting Mode190

Table 5.14: Input Sub-Module for the Mean Reversion with Jumps Price Forecasting Model 90

Table 5.15: Field Production Rate Allocation that Maximizes the Objective Function for Different Price Forecasting Models .......................................992

Table 6.1: $\quad$ Flat Price Values ............................................................................95

Table 6.2: $\quad$ Optimum Allocation Decision for Flat Price Model........................98

Table 6.3: $\quad$ Optimum Allocation Decision by Crude Grades for Flat Price Mode199

Table 6.4: Total NPV Change per \$1 Increase in Prices.................................101

Table 6.5: The Optimization Sensitivity Analysis of the XXLight Crude Price 102

Table 6.6: XLight Crude Price Optimization Sensitivity ................................ 103 xvi 
Table 6.7: $\quad$ Light Crude Price Optimization Sensitivity...................................104

Table 6.8: Medium Crude Price Optimization Sensitivity …..........................105

Table 6.9: Heavy Crude Price Optimization Sensitivity....................................106

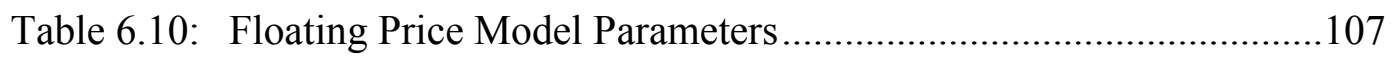

Table 6.11: Optimum Allocation for Floating Price Model.............................110

Table 6.12: Optimum Allocation by Crude Grade for Floating Price Model ....110

Table 6.13: XXLight Price Growth Rate Optimization Sensitivity ...................113

Table 6.14: XLight Price Growth Rate Optimization Sensitivity ......................114

Table 6.15: Light Price Growth Rate Optimization Sensitivity ........................115

Table 6.16: Medium Price Growth Rate Optimization Sensitivity ....................116

Table 6.17: Heavy Price Growth Rate Optimization Sensitivity .......................118

Table 6.18: The Optimum Allocation for ILD Price Forecast Model .................120

Table 6.19: The Optimum Allocation by Crude Grade for the ILD Price Forecast

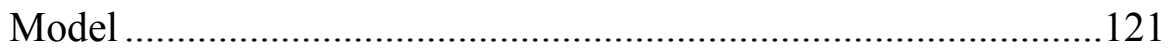

Table 6.20: Optimization Sensitivity Analysis of the Medium-Heavy Price Correlation Factor . 125

Table 6.21: Optimization Sensitivity Analysis of the Light-Heavy Price Correlation Factor

Table 6.22: Optimization Sensitivity Analysis of the XLight-Heavy Price Correlation Factor .

Table 6.23: Optimization Sensitivity Analysis of the XXLight-Heavy Price Correlation 127

Table 6.23: The GMB Optimum Allocation Decision......................................129

Table 6.24: The GBM Optimum Allocation Decision by Crude Grade ............129

Table 6.25: The MRM Optimum Allocation Decision ....................................135 xvii 
Table 6.26: The MRM Optimum Allocation Decision by Crude Grade.............136

Table 6.27: MRJM Optimum Allocation Decision..........................................141

Table 6.28: MRJM Optimum Allocation Decision by Crude Grade .................141

Table 6.29: Optimum Allocation Decision for Different Price Forecast Models 148

Table 6.30: Total NPV, Mean of the Total NPV, and Standard Deviation of Total NPV for Different Price Forecast Models ..................................149

Table 7.1: The Optimum Production Allocation Decisions for the Base and Alternate Cases of Heavy-Medium Price Correlation Factor .........153

Table 7.2: The Optimum Production Allocation Decisions for the Base and Alternate Cases of Heavy-Light Price Correlation Factor ..............156

Table 7.3: The Optimum Production Allocation Decisions for the Base and Alternate Cases of Heavy-XLight Price Correlation Factor ...........158

Table 7.4: The Optimum Production Allocation Decisions for the Base and Alternate Cases of Heavy-XXLight Price Correlation Factor ........161 


\section{List of Figures}

Figure 2.1: The Historical Data of Crude Oil Prices ................................................

Figure 2.2: The Probability Distribution of the Light Crude..................................6

Figure2.3: The Probability Distribution of the Medium Crude.............................6

Figure 2.4: The Probability Distribution of the Heavy Crude ………………….....

Figure 2.5: The Historical Data of the Price Spreads for Light-Medium, and Medium-Heavy Crudes .....................................................................12

Figure 2.6: The Spread Probability Distribution for the Light and Medium Crude

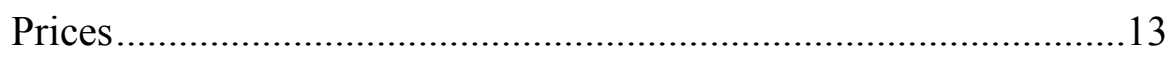

Figure 2.7: The Spread Probability Distribution for the Medium and Heavy Crude

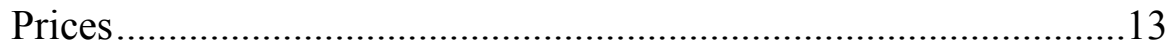

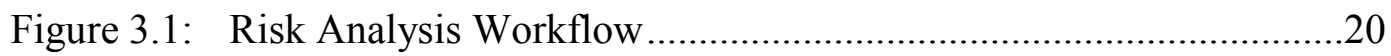

Figure 3.2: Hypothetical Price Forecast ……………....................................20

Figure 3.3: Production Allocation in Response to Figure 3.2 Price Forecast, which reveals the anticipatory error

Figure 3.4: Optimum Production Allocation as a Single Value Eliminating the

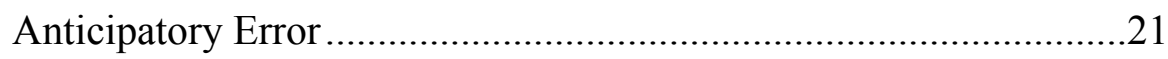

Figure 4.1: The Historical Data of Crude Oil Price...............................................23

Figure 4.2: Different Long Run Equilibrium Crude Prices 1987-2012 _..............25

Figure 4.3: $\quad P^{*}$ and $\eta$ Estimation Using Regression Analysis (AR(1)) .................34

Figure 4.4: The Flat Price Model …………………….....................................36

Figure 4.5: The Floating Price Model ..............................................................

Figure 4.6: Independent Lognormal Distribution Forecast Model.......................41

Figure 4.7: Arithmetic Brownian Motion Samples (Dyer, 2011) ........................42 
Figure 4.8: Geometric Brownian Motion Samples (Dyer, 2011)

Figure 4.9: Log-normal Diffusion Process of the GBM, Source: www.pucrio.br/marco.ind/stochast.html .43

Figure 4.10: The GBM Forecasting Model 45

Figure 4.11: Mean Reversion Sample Path. 47

Figure 4.12: Brent Crude Monthly Prices (1970-2000), Source: www.pucrio.br/marco.ind/rev-jump.html .50

Figure 4.13: The Heavy and the Medium Crude Prices Correlation Using 1989-2010 Weekly Historical Data .56

Figure 4.14: The Heavy and Medium Price Correlation Using 1989-2010 Annual Historical Data .58

Figure 4.15: The Heavy and Light Crude Price Correlation Using 1989-2010 Annual historical Data 58

Figure 5.1: Optimization Process .............................................................63

Figure 5.2: Probability Density Function of Total NPV ................................64

Figure 5.3: The ILD Price Model ..............................................................66

Figure 5.4: Mean Reversion Model .........................................................66

Figure 5.5: Cash Flow Diagram of Development Field Projects ......................86

Figure 5.6: Cash Flow Diagram of Producing Field Project .............................87

Figure 6.2: Optimum Production Allocation Decision for the Flat Price Model 99

Figure 6.3: Total NPV Tornado Chart by Varying Parameters $\pm 10 \%$..............100

Figure 6.4: Price Impact on Total NPV within each Crude Grade....................100

Figure 6.5: Floating Price Model.............................................................. 108

Figure 6.6: Optimum Production Allocation Decision for Floating Price model111

Figure 6.7: Total NPV Tornado Chart by Varying Parameters $\pm 10 \%$..............112 $\mathrm{XX}$ 
Figure 6.8: The Impact of Price Growth Rates on the Total NPV ...................112

Figure 6.9: The ILD Price Forecast Model (200 iterations) ............................119

Figure 6.10: Optimum Production Allocation for the ILD Price Forecast Model121

Figure 6.11: Total NPV Probability Density Function Resulted from the ILD Price

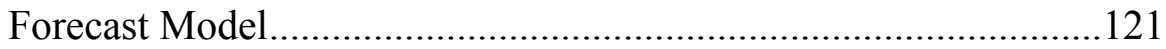

Figure 6.12: Total NPV Tornado Chart by Varying Parameters $\pm 10 \%$..............123

Figure 6.13: Sensitivity Analysis of Mean of Total NPV by Changing the Initial Heavy Crude Price 123

Figure 6.14: Sensitivity Analysis of Mean of Total NPV by Changing the Initial Heavy Crude Standard Deviation. 124

Figure 6.14: Sensitivity Analysis of Mean of Total NPV by Changing the Standard Deviation Growth Factor 124

Figure 6.15: The Optimum Allocation Decision the GBM...............................130

Figure 6.16: Total NPV Distribution of the GBM Model ................................130

Figure 6.17: Total NPV Tornado Chart by Varying Parameters $\pm 10 \%$..............131

Figure 6.18: Sensitivity Analysis of the Mean of Total NPV to the Drift of the Heavy Crude Price. 132

Figure 6.19: The GBM Price Model with “0” Price Drift (50 Iterations) ...........132

Figure 6.20: The GBM Price Model with “0.3” Price Drift (50 Iterations) ........132 Figure 6.21: Sensitivity Analysis of the Mean of Total NPV to the Volatility of the Heavy Crude Price..... 133

Figure 6.22: The GBM Price Model with “0.2” Price Volatility (50 Iterations).134 Figure 6.23: The GBM Price Model with “0.3” Price Volatility (50 Iterations).134

Figure 6.24: The MRM Optimum Allocation Decision ...................................136

Figure 6.25: Total NPV Distribution of the MRM Price Forecast Model ..........136 $\mathrm{xxi}$ 
Figure 6.26: Total NPV Tornado Chart by Varying Parameters $\pm 10 \%$

Figure 6.27: Sensitivity Analysis of the Mean of Total NPV to the Reversion Speed of the Heavy Price 138

Figure 6.28: The MRM with 5 Reversion Speed 138

Figure 6.29: The MRM with "0.1” Reversion Speed 139

Figure 6.30: Sensitivity Analysis of the Mean of Total NPV to the Long-run Mean price of the Heavy Crude 139

Figure 6.31: Sensitivity Analysis of the Mean of Total NPV to the Volatility of the Heavy Price 140

Figure 6.32: MRJM Optimum Allocation Decision.........................................142

Figure 6.33: MRJM Total NPV Probability Density Function ..........................142

Figure 6.34: Total NPV Tornado Chart by Varying Parameters $\pm 10 \%$ 143

Figure 6.35: Sensitivity Analysis of the Mean of Total NPV to the Long-run Mean Price of the Heavy Crude 144

Figure 6.36: Sensitivity of Mean of Total NPV to Jump Frequency 144

Figure 6.37: Sensitivity of Mean of Total NPV to Jump Size 145

Figure 6.38: Sensitivity of Total NPV to the mean of squared jump size 145

Figure 6.39: Optimum Production Allocation Decisions for Different Price Forecasting Models 148

Figure 7.1: Decision Tree to Evaluate the Value of Mitigating the Uncertainty in the Heavy-Medium Price Correlation Factor 153

Figure 7.2: Value of an Accurate Heavy-Medium Correlation Model as a Function of Correlation Factor 154

Figure 7.3: Decision Tree to Evaluate the Value of Mitigating the Uncertainty in the Heavy-Light Price Correlation Factor 155 xxii 
Figure 7.4: Value of an Accurate Heavy-Light Correlation Model as a Function of Correlation Factor 156

Figure 7.5: Decision Tree to Evaluate the Value of Mitigating the Uncertainty in the Heavy-XLight Price Correlation Factor. 158

Figure 7.6: Value of an Accurate Heavy-XLight Correlation Model as a Function of Correlation Factor 159

Figure 7.7: Decision Tree to Evaluate the Value of Mitigating the Uncertainty in the Heavy-XXLight Price Correlation Factor..... 160

Figure 7.8: Value of an Accurate Heavy-XXLight Correlation Model as a Function of Correlation Factor. 161 


\section{CHAPTER 1: INTRODUCTION}

Oil companies, especially international oil companies (IOC), produce at maximum potential to recover expenses, pay off loans and develop new fields. This strategy reveals the importance of "Time Value of Money". On the other hand, national oil companies (NOCs), especially major producer companies, are subject to additional influences. The fact that NOCs are governmental companies plays a major role in deciding production strategy; political factors intervene in these decisions. Mega producer national oil companies reserve excess production for emergencies, political events and other governmental needs. Despite the fact that a NOC is a governmental company, a main goal is to maximize profit. Consequently, the price of crude is a vital factor in production allocation decisions. The impact of price multiplies in the case of NOC producing different crude grades, especially when the price differentials vary. Campbell et al. (2001) and Olsen et al. (2005) find that the price of crude is the most significant parameter in E\&P project valuation.

Since 2004 , crude prices have been extremely volatile. This volatility has affected decision making in the oil industry (capital investment, production optimization, etc.), and encouraged the oil industry to invest heavily in decision making research. This change in orientation is a result of the impact of crude price volatility on mega oil projects that cost billions of dollars. Allocating production volumes across a portfolio of producing assets is a complex optimization problem and a critical decision. Specifically, each producing asset possesses different technical attributes (e.g. crude type), facility constraints, and costs. In addition to these field-level specifications, there are corporate objectives and constraints (e.g. delivery contracts). While complex, such a problem can be defined and solved using conventional deterministic optimization methods. However, 
the problem is complicated by the presence of uncertainty in many of the inputs. One of the major uncertainties is the commodity price assumption(s).

Crude price forecasting is an estimation of future crude prices to make an informed investment decisions today (MacAskie, 2007). Oil companies are investing in crude price modeling research and development believing that the more sophisticated the forecast, the better the results and the higher the profit. Assuming that Future Prices are the market's best estimate of future crude prices, Stephan (2006) proposed an improved Mean Reversion process where crude prices revert to Future Prices. Stephan stated that "for a realistic E\&P project valuation it is critical to use sophisticated methods for the estimation of the future oil price". In this proposed process, crude prices follow a predefined path instead of a mean equilibrium price. Faya et al. (2007) implemented an improved Mean Reversion process to forecast crude prices in a portfolio optimizing model where limited funds are assigned among exploration and development projects; this study includes reservoir uncertainties. This enhancement of the price forecasting model involved adding a growth rate factor to the mean equilibrium price. Crude prices reverted to increasing mean price equilibrium. Hahn and Dyer (2005) proposed a new method for modeling Mean Reversion processes that enables straightforward binomial tree and lattice-based approaches to valuation. The proposed process is a computationally efficient model. The computational load is reduced by implementing a discrete approximation of the underlying stochastic process.

Sophisticated price forecasting models have been developed in the last few decades, but few studies were devoted to model comparisons. Metcalf and Hasset (1995) concluded that the cumulative investment is unaffected by the use of the more sophisticated Mean Reversion Model in contrast to the simpler Geometric Brownian Motion. Although much research has been done on building and enhancing complex 
price models, rather less attention has been paid to relating the price modeling assumptions (model type and the respective parameters) to the decisions that result from their use (capital investment, production optimization, etc.). No one appears to have asked the basic question, "Do any of these models provide an incremental value for decision-making?" Here, we address this question by developing an integrated stochastic optimization model that simulates decision-maker behavior. Using this model, we compare and contrast the various production allocation decisions that result from different price models. Simple price models are investigated and compared to several popular advanced price models with the goal of understanding the impact of price model assumptions (both the model type and its parameters) on decision-making.

Our results show that the optimum production allocation is a function of the price model assumptions. However, the differences between models are minor, and thus the value of choosing the "correct" price model, or of estimating a more accurate model, is negligible for this specific decision.

The outline of this thesis is as follows: Chapter 2 presents general information on the crude grades and prices involved in the study including background on crude classifications, historical data on prices and crude price distribution properties. Chapter 3 discusses the risk analysis workflow, which is implemented in the study. This chapter covers step by step the workflow used in the study. Chapter 4 presents the deterministic and probabilistic price forecasting models; moreover, the chapter presents the correlation models to correlate the price of different crudes. Chapter 5 discusses the modeling and formulation of the integrated optimization model. This chapter covers the model objectives, assumptions, description, and formulations. Chapter 6 presents the results, analysis, and the discussion. Chapter 7 lists the conclusions of the study and list recommendations for future studies. 


\section{CHAPTER 2: CRUDE AND PRICING GENERAL INFORMATION}

\subsection{Crude General Information}

The research studies 23 fields that produce 5 different crude grades classified by API gravity as illustrated in Table 2.1 below:

Table 2.1: Crude Grade Classification

\begin{tabular}{|lcc|}
\hline \multicolumn{1}{|c}{ Crude Type } & Gravity $\left({ }^{\circ}\right.$ API) & Producing Fields \\
\hline XX-Light Grade & $50-52$ & 3 \\
X-Light Grade & 39.5 & 4 \\
Light Grade & 34 & 8 \\
Medium Grade & 31 & 4 \\
Heavy Grade & 27 & 4 \\
\hline \hline \multicolumn{2}{|c|}{ Total } \\
\hline
\end{tabular}

The Light crude fields dominate the total production, which can be seen from the number of producing fields within each crude grade in Table 2.1.

Crude oil prices historical data are obtained for only three crude grades (Heavy, Medium, and Light) from the U.S. Energy Information Administration (www.eia.deo.gov). The weekly crude prices obtained covered the period between 1978 and 2010 as shown in Figure 2.1.

Using the EXCEL functions Average and STDEV, the mean and standard deviation of the three available crudes were calculated. These properties are essential for forecasting crude prices probabilistically. The properties of the two missing crudes were estimated by assuming that these properties increases at fix rate per 1 API increase. Table 2.2 lists these probability distribution properties. 
Table 2.2: $\quad$ Crudes Probability Distribution Properties

\begin{tabular}{|lccccccc|}
\hline Crude Type & Gravity ( ${ }^{\circ}$ API) & Distribution Type & Mean (\$\$) & Standard Deviation (\$) & Min (\$) & Max (\$) & Remarks \\
\hline XX-Light & 52 & Lognormal & 44.06 & 88.94 & 10.56 & 148.00 & Assumed \\
X-Light & 50 & Lognormal & 42.69 & 63.02 & 10.00 & 141.00 & Assumed \\
Light & 34 & Lognormal & 34.41 & 44.89 & 9.65 & 136.02 & Historical Data \\
Medium & 31 & Lognormal & 33.36 & 42.95 & 9.25 & 131.77 & Historical Data \\
Heavy & 27 & Lognormal & 32.63 & 42.31 & 8.50 & 128.72 & Historical Datata \\
\hline
\end{tabular}

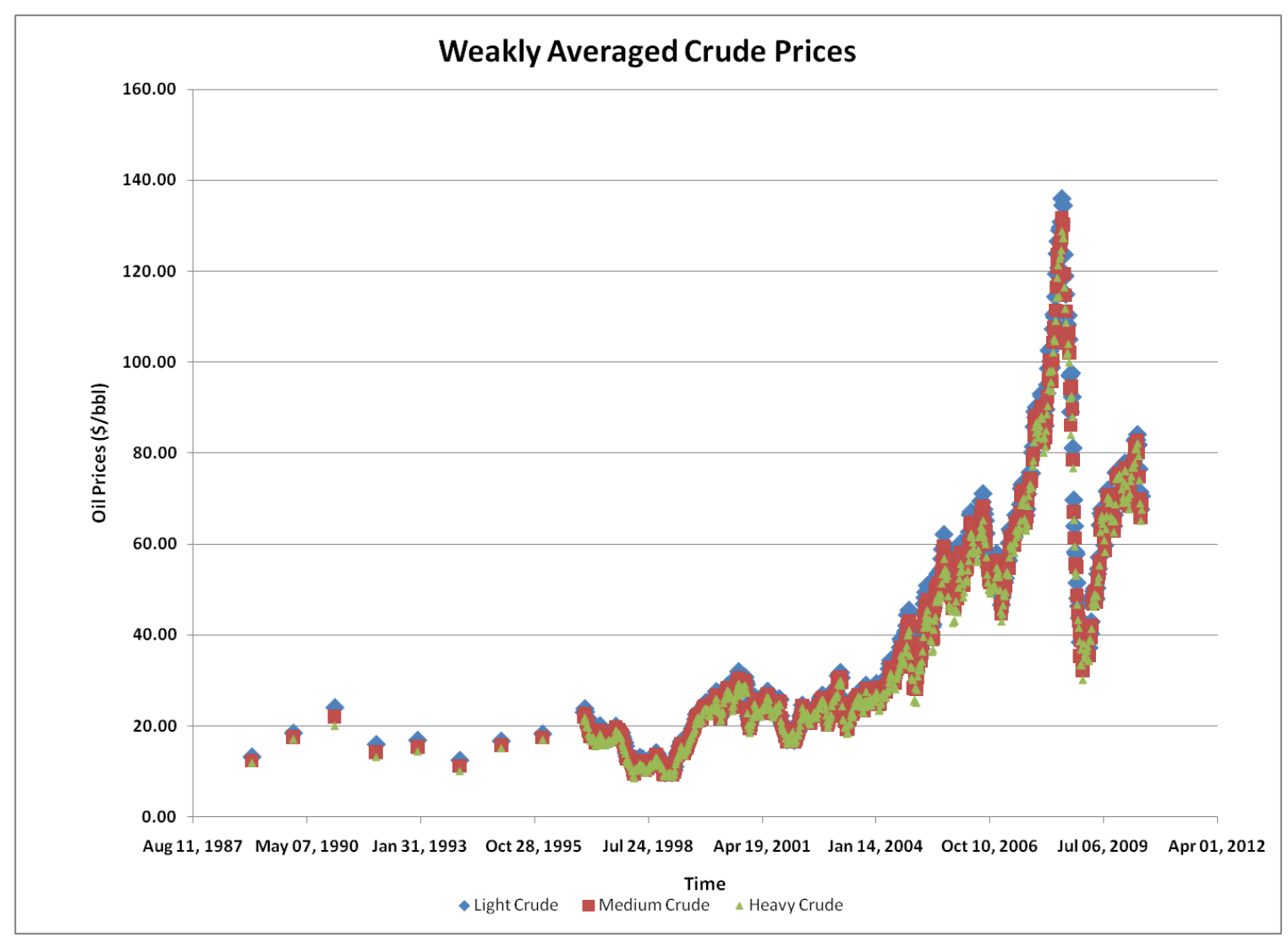

Figure 2.1: The Historical Data of Crude Oil Prices

Figures 2.2, 2.3, and 2.4 show the historical price data fitted as a probability distribution for the Light, Medium, and Heavy crudes, respectively. The three crude grades fit a lognormal distribution with the properties shown in Table 2.3. 


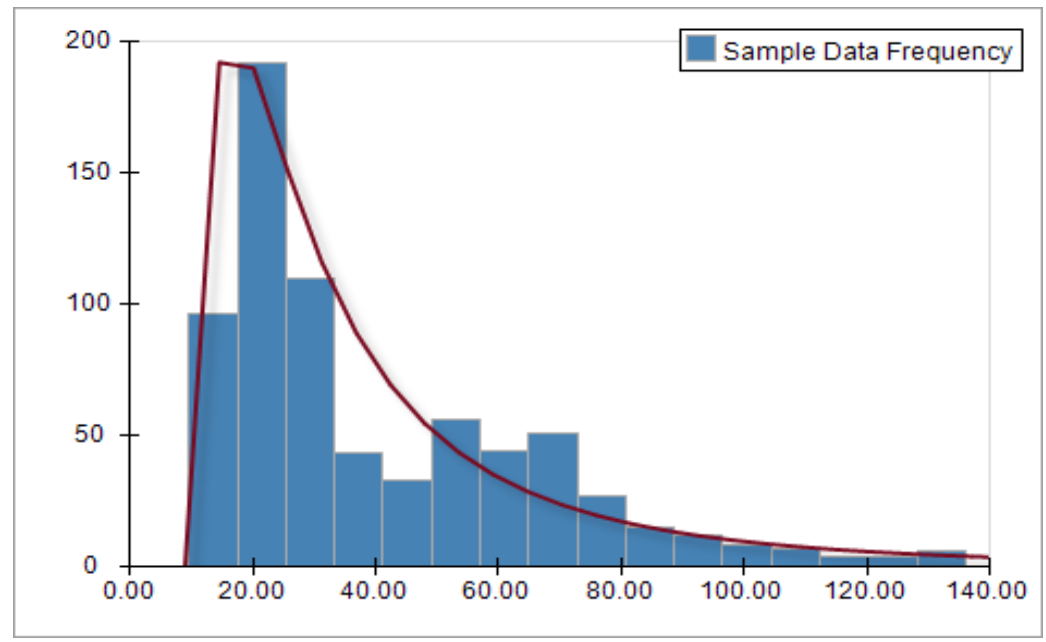

Figure 2.2: The Probability Distribution of the Light Crude

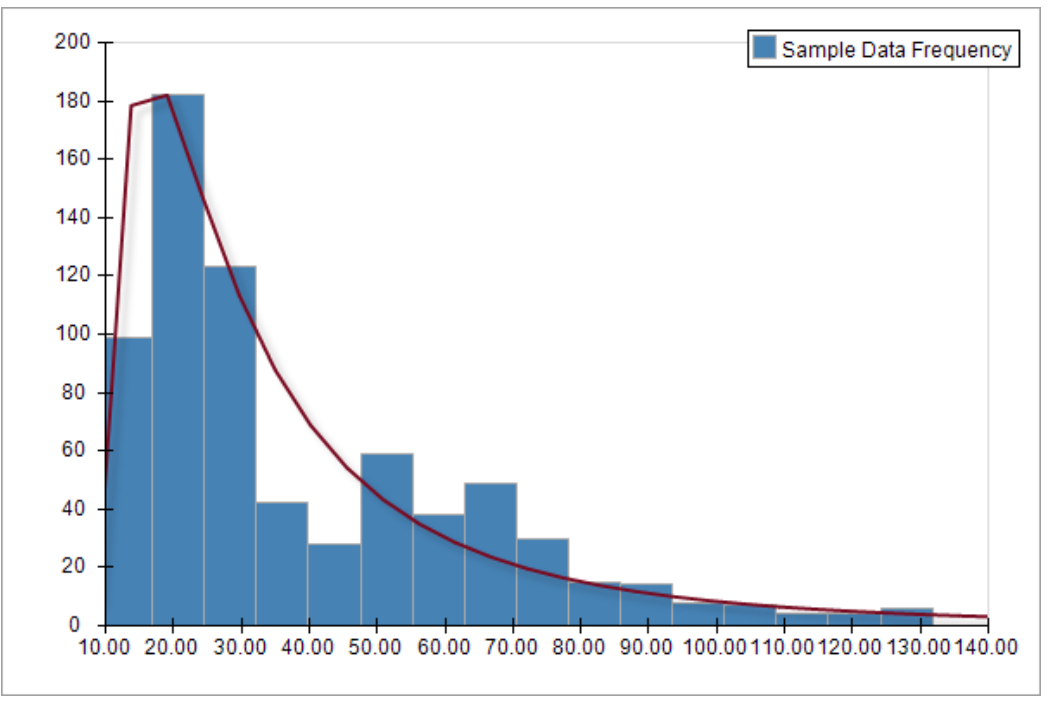

Figure2.3: The Probability Distribution of the Medium Crude 


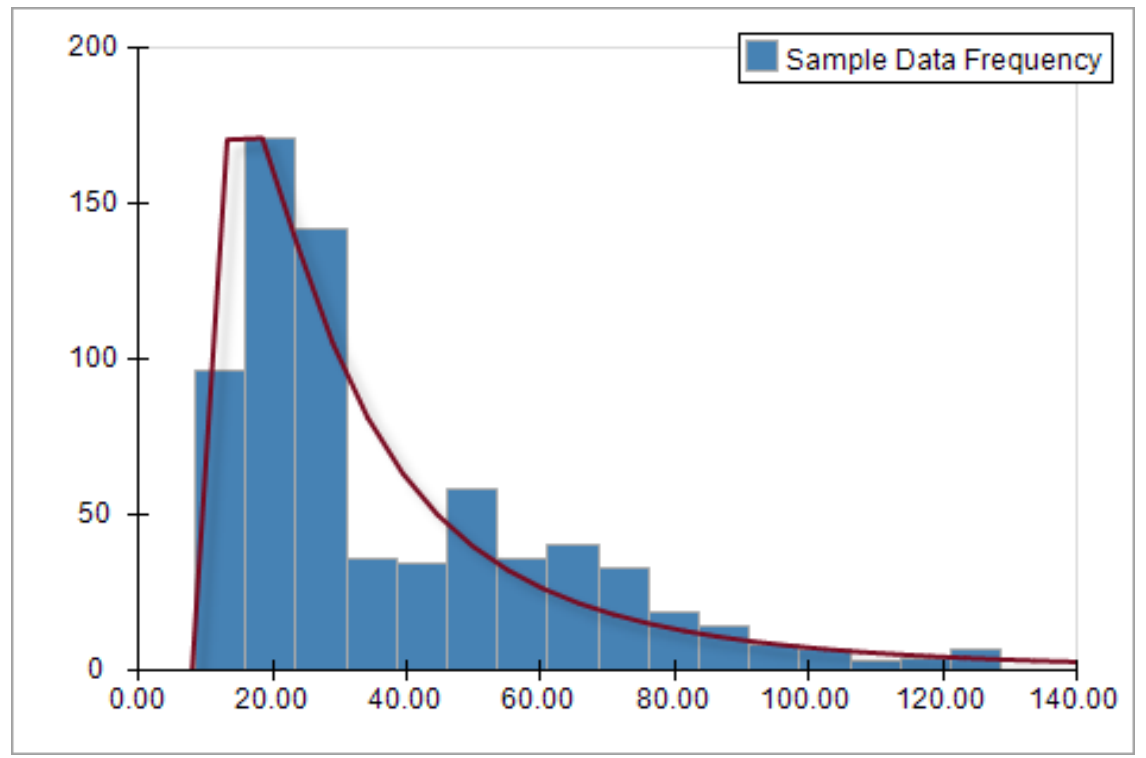

Figure 2.4: The Probability Distribution of the Heavy Crude

Table 2.3: Properties of the Probability Distributions of the Available Crude Oil Price Data

\begin{tabular}{|llcccc|}
\hline \multicolumn{1}{|c}{ Crude Type } & Distribution Type & Mean & Standard Deviation & Min & Max \\
& & & & & \\
\hline Light & Lognormal & 34.41 & 44.89 & 9.65 & 136.02 \\
Medium & Lognormal & 33.36 & 42.95 & 9.25 & 131.77 \\
Heavy & Lognormal & 32.63 & 42.31 & 8.5 & 128.72 \\
\hline
\end{tabular}

\subsection{Estimating the Properties of Crude Price Distribution}

As mentioned earlier, crude price historical data were obtained for three crude grades: Heavy, Medium, and Light. The data were fitted through probability distribution shown in Figures 2.2, 2.3, and 2.4 with properties in Table 2.3. The distribution properties are essential in forecasting crude price using stochastic processes. This section illustrates the procedure of estimating these properties for the two missing crudes (XLight \& XX-Light). 


\subsubsection{Mean Price and Standard Deviation Estimation}

The mean and standard deviation for the two missing crudes were estimated using the data of the available crudes. Crude prices increases as crude grade increases (lighter crude or higher API gravity). In other words, crude oil prices and the API gravity of the crude are correlated. Consequently, the distribution properties of the missing crudes are estimated by determining the incremental value of the mean price and standard deviation and add them to the ones of the lower grade.

As mentioned above, the crude grades are classified based on their API gravity. Since the API gravity are known for all the crudes, and the distribution properties are known for three crudes, we estimated the incremental value for the distribution properties for 1 API increase in the crude gravity, for all the missing crudes. Then, these incremental values are added to the properties of the known crudes to obtain the properties of the unknown crudes; keep in mind that the incremental values are multiplied by the API differential of the two crudes before adding it to the properties of the known crudes. As shown in Table-2.4, the additional value of the mean price, resulted from 1 API increase, increases as the crude grade increases. In other words, any additional API is more valuable or has greater impact on the distribution properties than the previous API. For example, as crude goes from Heavy to Medium, the mean price increases by $\$ 0.18$ per 1 API increment and it increases by $\$ 0.35$ as the crude goes from Medium to Light. Similarly, the standard deviation of price distribution increases as the crude becomes lighter. The estimation approach of the mean price and standard deviation for the unknown crudes (X-Light and XX-Light) assumes that as the crude grade gets lighter, the mean price and standard deviation increase at higher rate per API increase. To calculate the incremental values of mean price and standard deviation per API increase as crude goes from Light to X-Light and from X-Light to XX-Light, it is assumed that the 
incremental rates of mean price and standard deviation are fixed per 1 API increase. Mean price and standard deviation incremental rate per one API increase are calculated from the available data as 0.17 and 0.4 respectively.

\subsubsection{Estimation of Mean Price}

First, the rate of increase in mean price per 1 API increase is calculated as crude grade goes from Heavy to Medium and from Medium to Light as illustrated in Equation 2.1; $\Delta$ Mean Price Increment is the difference of the two rates of increase (Equation 2.2). The rates of increase in mean price per 1 API increase, for the missing crudes, are calculated by adding the $\Delta$ Mean Price Increment (calculated from the available crudes) to the rate of increase in mean price of the heavier crude (Equation 2.3). Equation 2.4 shows the final step to calculate the mean price of the missing crudes, adding the rate of increase in mean price to the mean price of the heavier crude (Table 2.5).

$$
\begin{aligned}
& M P I(c, " c+1 ")=\frac{(\mu P(c)-\mu P(c+1)}{(G V(c)-G V(c+1)} \\
& \triangle M P I=M P I(L-M)-M P I(M-H) \\
& M P I(M C)=M P I(M C+1)+\Delta M P I \\
& \boldsymbol{\mu P}(M C)=M P(M C-1)+[M P I(M C) *(G V(M C)-G V(M C-1)]
\end{aligned}
$$

Where:

$\mathrm{C}=$ Crude Grade Number, $\mathrm{c}=1,2 \ldots \mathrm{C}$ (Illustrated in model formulation section)

$\operatorname{MPI}(\mathbf{c}, " \mathbf{c}+\mathbf{1} ")=$ Mean Price Increment per 1 API increase of crude c and heavier crude $\mathrm{c}+1$

MPI(MC) = Mean Price Increment per 1 API increase of the missing crude

MPI $(\mathbf{M C}+\mathbf{1})=$ Mean Price Increment of heavier crude than missing crude

$\Delta \mathbf{M P I}=$ Difference of Mean Price Increment

MPI (L-M) = Mean Price Increment between medium and light crudes 
MPI (M-H) = Mean Price Increment between medium and heavy crudes.

$\boldsymbol{\mu P}(\mathbf{M C})=$ Mean Price of missing crude.

$\mathbf{G V}(\mathbf{M C})=$ API Gravity of missing crude

\subsubsection{Estimation of Standard Deviation}

First, the rate of increase in the standard deviation of price per 1 API increase is calculated as crude grade goes from Heavy to Medium and from Medium to Light as illustrated in Equation 2.5; $\Delta$ Standard Deviation Increment is the difference of the two rates of increase (Equation 2.6). The rates of increase in standard deviation per 1 API increase, for the missing crudes, are calculated by adding the $\Delta$ standard deviation Increment (calculated from the available crudes) to the rate of increase in standard deviation of the heavier crude (Equation 2.7). Equation 2.8 shows the final step to calculate the standard deviation of the missing crudes, adding the rate of increase in standard deviation to the standard deviation of the heavier crude (Table 2.5).

$$
\begin{aligned}
& S D I(c, " c+1 ")=\frac{(S D(c)-S D(c+1)}{(G V(c)-G V(c+1)} \\
& \triangle S D I=S D I(L-M)-S D I(M-H) \\
& S D I(M C)=S D I(M C+1)+\Delta S D I \\
& S D(M C)=S D(M C-1)+[S D I(M C) *(G V(M C)-G V(M C-1)]
\end{aligned}
$$

Where:

$\mathrm{C}=$ Crude Grade Number, $\mathrm{c}=1,2 \ldots \mathrm{C}$ (Illustrated in model formulation section)

SDI $(c, " c+1 ")=$ Standard Deviation Increment per 1 API increase of crude "c" and heavier crude "c+1"

$\mathrm{SDI}(\mathrm{MC})=$ Standard Deviation Increment per 1 API increase of the missing crude $\mathrm{SDI}(\mathrm{MC}+1)=$ Standard Deviation Increment of heavier crude than missing crude $\Delta \mathrm{SDI}=$ Difference of Standard Deviation Increment 
SDI $(\mathrm{L}-\mathrm{M})=$ Standard Deviation Increment between medium and light crudes

SDI $(\mathrm{M}-\mathrm{H})=$ Standard Deviation Increment between medium and heavy crudes

$\mathrm{SD}(\mathrm{MC})=$ Standard Deviation of missing crude

GV $(\mathrm{MC})=$ API Gravity of missing crude

Table 2.4: Crude Mean Price and Standard Deviation Calculations for the Missing Crudes

\begin{tabular}{|lccccccc|}
\hline Crude Type & Gravity ('API) & Distribution Type & $\begin{array}{c}\text { Mean Increment } \\
\text { Per API (\$\$/API) }\end{array}$ & $\begin{array}{c}\text { Standard Deviation } \\
\text { Increment Per API } \\
\text { (\$\$/API) }\end{array}$ & $\begin{array}{c}\text { Mean Increment Standard Deviation } \\
\text { Per API } \\
\text { Difference }\end{array}$ & $\begin{array}{c}\text { Increment Per API } \\
\text { Difference }\end{array}$ & Remarks \\
\hline XX-Light & 52 & Lognormal & 0.68 & 1.62 & 0.17 & 0.49 & Assumed \\
X-Light & 50 & Lognormal & 0.52 & 1.13 & 0.17 & 0.49 & Assumed \\
Light & 34 & Lognormal & 0.35 & 0.65 & 0.17 & 0.49 & Calculated \\
Medium & 31 & Lognormal & 0.18 & 0.16 & - & - & Calculated \\
Heavy & 27 & Lognormal & - & - & - & - & Calculated \\
\hline
\end{tabular}

Table 2.5: The Properties of the Probability Distribution for the Crude Oil Prices

\begin{tabular}{|lccccccc|}
\hline Crude Type & Gravity ( ${ }^{\circ}$ API) & Distribution Type & Mean (\$) & Standard Deviation (\$) & Min (\$) & Max (\$) & Remarks \\
\hline XX-Light & 52 & Lognormal & 44.06 & 88.94 & 10.56 & 148.00 & Assumed \\
X-Light & 50 & Lognormal & 42.69 & 63.02 & 10.00 & 141.00 & Assumed \\
Light & 34 & Lognormal & 34.41 & 44.89 & 9.65 & 136.02 & Historical Data \\
Medium & 31 & Lognormal & 33.36 & 42.95 & 9.25 & 131.77 & Historical Data \\
Heavy & 27 & Lognormal & 32.63 & 42.31 & 8.50 & 128.72 & Historical Data \\
\hline
\end{tabular}

\subsection{Crude Prices Spread}

Figure 2.5 presents the historical spread (1987-2011) of the available crude grades; the presented spreads are for Light-Medium, and Medium-Heavy crudes. The blue curve is the difference of the crude prices of the Light and Medium while the red curve is the difference for Medium and Heavy crudes. Note the spreads of the Light and the Medium crudes are greater than or equal to the ones of the Medium and Heavy. Moreover, the spread is a random variable and is not proper to forecast prices for one crude grade and use fixed spread to correlate the prices for the remaining grades. A suggested forecasting approach, which is not implemented in this study, is to forecast the 
price for a crude grade; then, use the price spread probability distributions (Figures 2.6 and 2.7) to correlate the prices of the remaining crudes. Table 2.6 lists the properties of the probability distribution for the spread of crude oil prices.

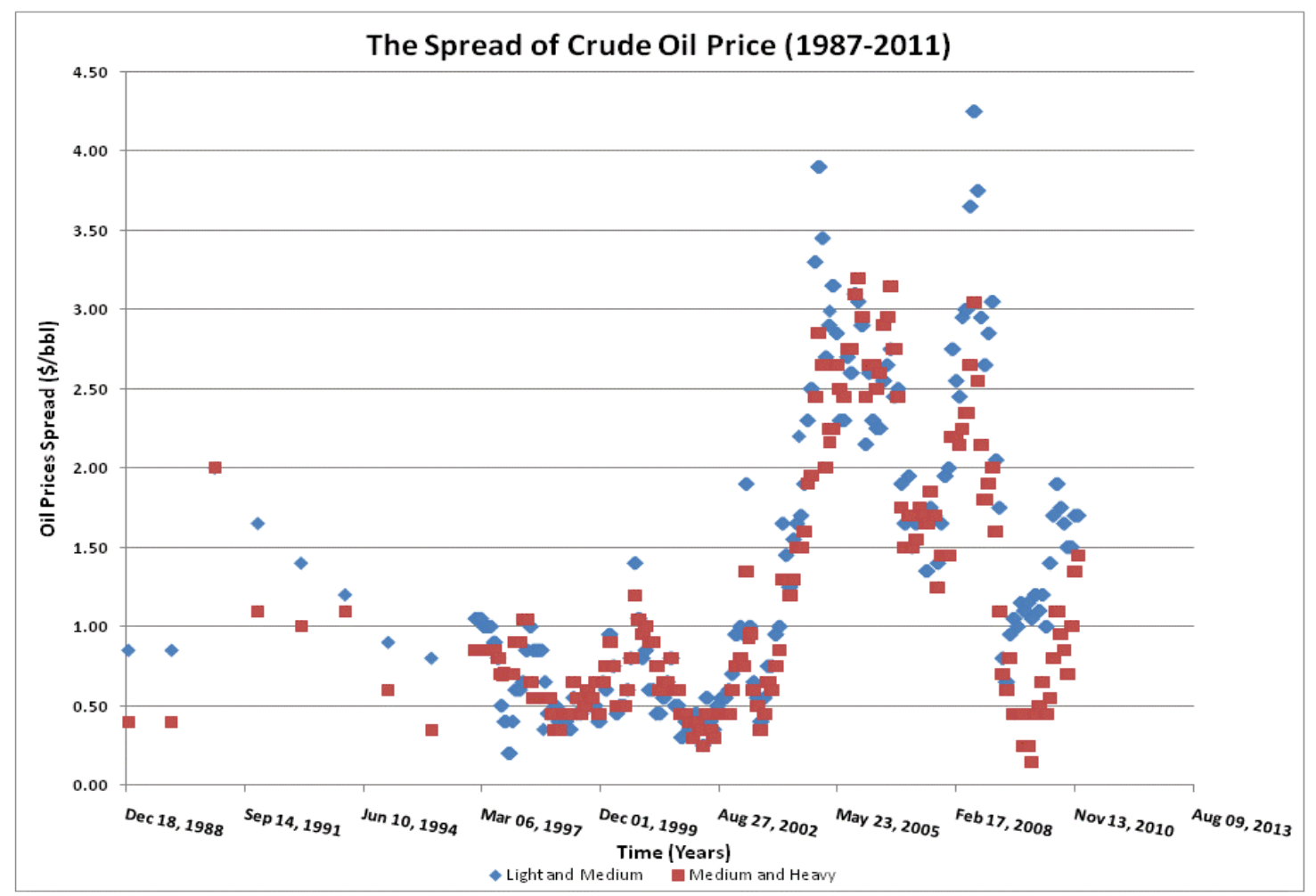

Figure 2.5: The Historical Data of the Price Spreads for Light-Medium, and MediumHeavy Crudes 


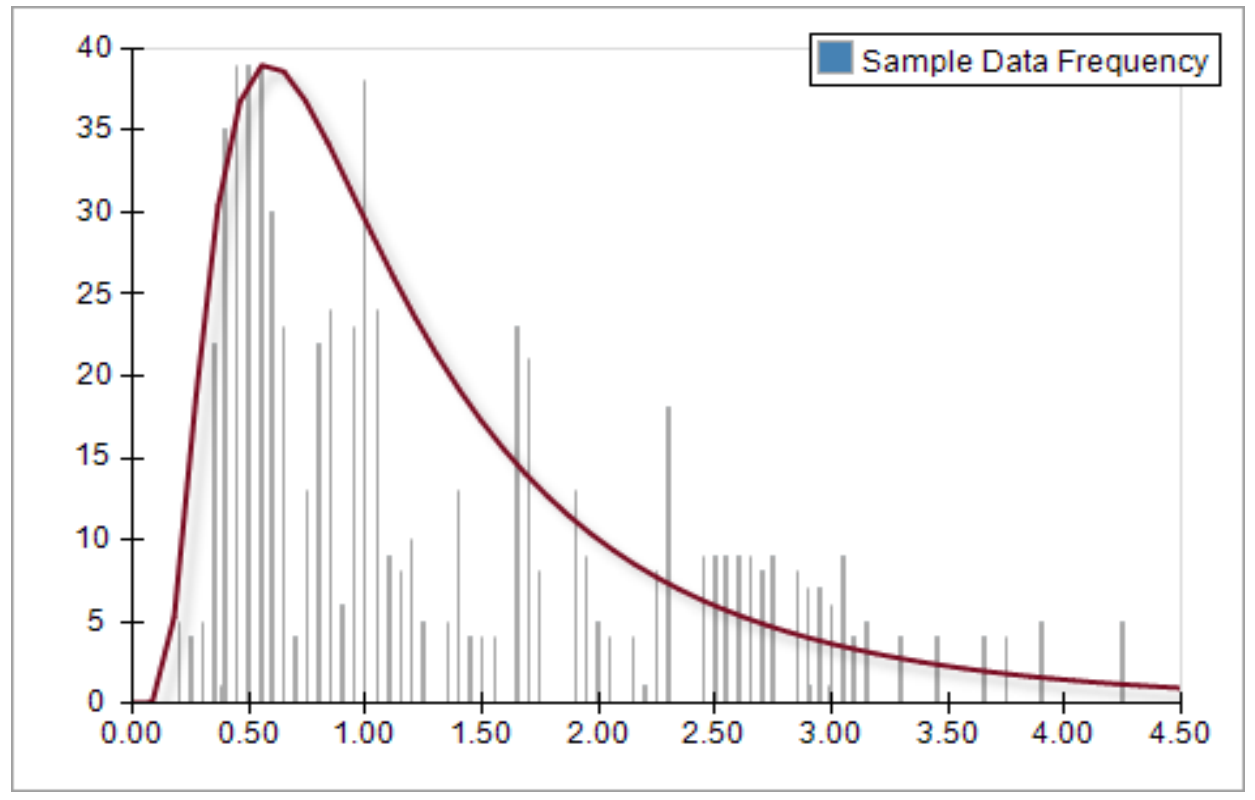

Figure 2.6: The Spread Probability Distribution for the Light and Medium Crude Prices

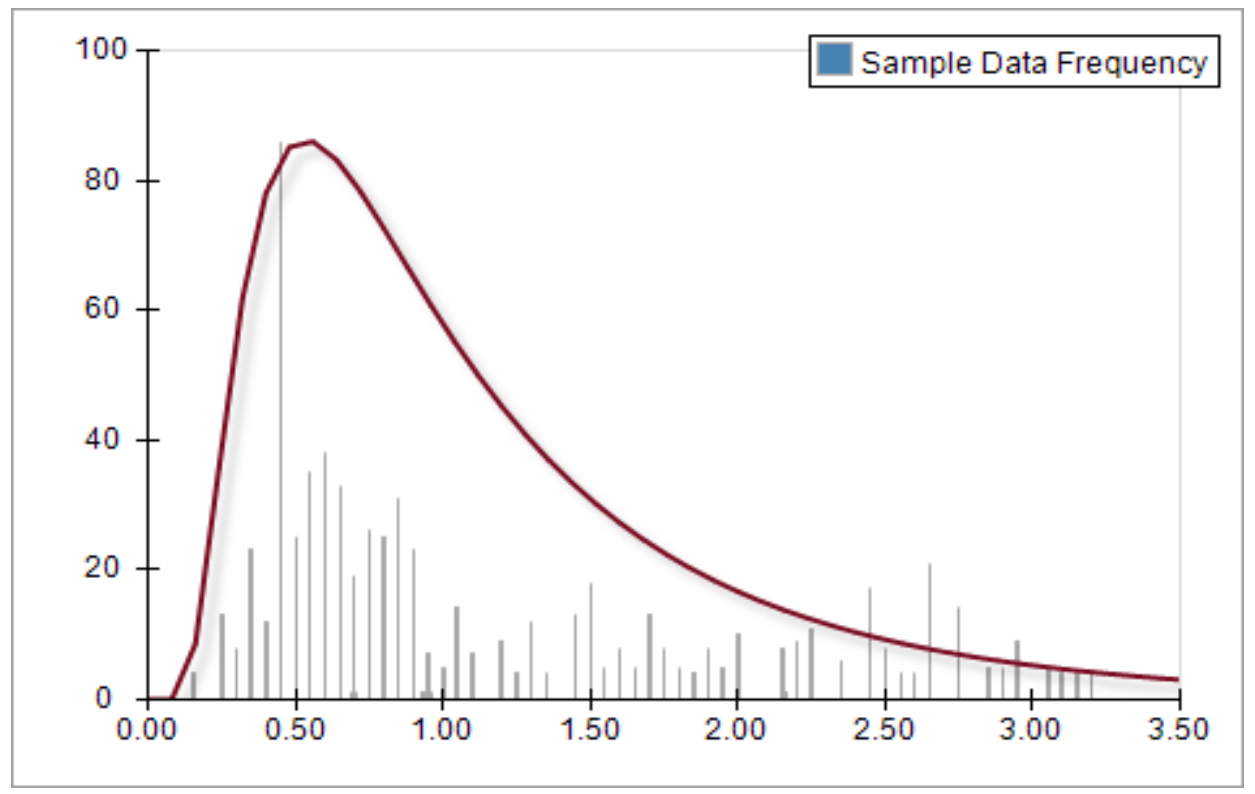

Figure 2.7: The Spread Probability Distribution for the Medium and Heavy Crude Prices 
Table 2.6: The Properties of Probability Distribution for the Spread of the Crude Oil Prices

\begin{tabular}{|lcccccc|}
\hline \multicolumn{1}{|c}{ Crude Type } & Distribution Type & Mean (\$̦) & Standard Deviation (\$) & Min (\$) & Max (\$) & Remarks \\
\hline "XX-Light" - "X-Light" & - & - & - & 0.37 & 6.35 & Estimated \\
"X-Light" - "Light" & - & - & - & 0.28 & 5.30 & Estimated \\
"Light" - "Medium" & Inverse Gaussian & 1.36 & 1.0767 & 0.20 & 4.25 & Actual Data \\
"Medium" - "Heavy" & Inverse Gaussian & 1.19 & 0.9196 & 0.15 & 3.20 & Actual Data \\
\hline
\end{tabular}




\section{CHAPTER 3: RISK ANALYSIS WORKFLOW}

The objective of the study is to identify the value added by sophisticated crude oil price models to the optimization problem defined in the introduction. This chapter describes the risk analysis workflow, which is used to achieve the objective. A similar approach to the analysis workflow was used to assess facility optimization and "value of information" problems for oil and gas producing fields by Jablonowski et al. (2008), Purwar (2008), Ettehad et al. (2009) and Hosgor (2009).

Risk analysis involves 8 steps, which are discussed in detail, and summarized in Figure 3.1.

\subsection{Define Optimization Approach}

There are two conventional optimization approaches to specifying gas storage facility design problems: asset-based and requirement based (Ettehad, 2009, and Mc Vay and Spivey, 2001). These two approaches are applicable for our optimization problem. The asset-based approach assumes that the price of oil is known, and then maximizes a performance attribute (for example, net present value) by modifying the fields' production rates. On the other hand, the requirement-based approach, which is implemented in this study, has a functionality requirement. In the approach we calculate the fields' production rate allocation that maximizes total NPV while meeting the functionality requirement, "total daily production rate". Ettehad (2009) implemented the requirement-based approach to optimize gas storage facility design under uncertainty.

In practice, a company's total production rate (functionality requirement) is determined by executive management. The optimization model design gives management the flexibility to assign the total production rate, and then calculates the production rate 
allocation among the various crude fields and thus maximizes the NPV while meeting the total target rate.

\subsection{Identify Functionality Requirements}

Functionality requirement is a constraint that must be satisfied by the optimization model for the model to be functional. In this study, functionality requirement was defined as the total target production rate. This functionality requirement represents the executive management's commitment to provide the agreed upon amount of crude in a timely manner. Functionality requirements can be defined as deterministic or probabilistic values. Ettehad (2009) used the probabilistic functionality requirement in a gas storage facility design optimization process to model demand more realistically because he used injection and production rates within the gas storage as functionality requirements. However, in this study, the functionality requirement was used as a deterministic single value specified by executive management. This decision reflects management's role in oil companies where the amount of crude oil to be sold and delivered is known and decided months in advance. In other words, most oil companies know the exact amount of oil to be produced on daily basis.

\subsection{Select Price Forecasting Approach}

The objective of the research is to compare and contrast the impact of deterministic and probabilistic price modeling approaches for production allocation decision making. After total target rate (functionality requirement) is determined for the integrated model, the user must choose the price forecasting approach and model. Our

study investigates 6 price forecasting models under the two forecasting approaches. Price forecasting approaches and models are discussed in detail in the Price Modeling chapter. 


\subsection{Initiate Production Rate Allocation}

Production rate allocation values are the decision variables of the optimization problem. Any optimization problem, especially a non-linear programming problem, requires initial values for the decision variables (The "Non-linear Programming" section in the Technical Background chapter discusses the functionality of the optimization algorithm in detail). As a result, the user must specify initial production allocation values for the model to function.

In practice, fields are developed and production facilities are constructed before crude prices are known. Production facility design (capacity) is a critical decision because it is a long-term decision and cannot be easily modified. Moreover, crude price contracts are sold a few months in advance, which means that crude production is determined months in advance of the delivery date. These facts impose a constraint on our optimization process since field production rates are assigned months before the price of oil is known. As a result, the constraints of the optimization problem must be defined before the revelation of the uncertainty variables (crude prices).

\subsection{Reveal Uncertainty Variables}

During the optimization process, the revelation of a single iteration of the uncertain variables (crude oil prices) occurs at the beginning of the process. In other words, the prices of crudes are revealed to the optimizer before the optimizer calculates the optimum decision variables. However, in reality, prices are revealed after production rates are assigned, which imposes uncertainty and risk to the problem. This sequence of events leads to anticipatory error as described by Ettehad (2009). Although our study followed the same sequence of events in the optimization process of Ettehad (2009), our integrated model was programmed in such a way that the anticipatory error did not affect 
the results. The anticipatory error was eliminated by designing field production rates (decision variables), so that they do not respond to the sampled, changing crude oil prices. Production rate is a fixed value for each field throughout the life of the project. In other words, the optimizer assigns a single value for each field throughout the life of the project. By implementing this strategy, the optimizer cannot respond to the changes in the crude oil price within one price iteration. For example, if an iteration of crude oil prices is as shown in Figure 3.2, the typical production allocation by the optimizer should be as expressed in Figure 3.3. From Figures 3.2 and 3.3, it is clear that the optimizer is aware of the price forecast, which is used to assign the optimum rate. In practice, crude oil prices are revealed after the field production rates are assigned. As a result, Figures 3.2 and 3.3 involve the anticipatory error, which is known as error of perfect information because the optimizer solves the problem with perfect knowledge of future prices. On the other hand, if the optimizer in our study implements the crude oil price in Figure 3.2, the optimum production rate would be a single value as shown in Figure 3.4 eliminating the anticipatory error.

\subsection{Maximize the Objective Function}

An optimization problem consists of 3 parts: objective function, decision variables, and constraints. The optimization algorithm changes decision variables to maximize the objective function while satisfying the constraints. Objective function is a performance attribute, which is used to assess the performance of a process. Two types of objective function were used in the study. Total NPV was used as objective function in the deterministic price forecasting approach. Total NPV is the sum of the NPVs of all fields. NPV is the most dominant performance attribute used in oil and gas projects planning. On the other hand, the total NPV in a probabilistic price forecasting approach is 
a probability density function PDF (probability distribution); the total NPV is not a single value that can be maximized. As recommended by Powell and Baker (2010), the objective function should be used as the mean of the total NPV PDF. To summarize, total NPV and mean of total NPV PDF are objective functions for deterministic and probabilistic price forecasting approaches, respectively.

\subsection{Generate Probability Density Function for the Objective Function}

During the optimization process in the probabilistic approach, the integrated optimization model retrieves price samples from the probability density function for each year and calculates the total NPV. This sampling process is repeated 1000 times to generate the probability density function for the total NPV.

\subsection{Solve for the Optimum Production Allocation}

The probability density function of the total NPV calculated in the previous step is for one set of decision variables. In this step, we set the integrated model to change the decision variables and solve to obtain total NPVPDF with maximum mean for the probabilistic approach, and total NPV for the deterministic approach. 


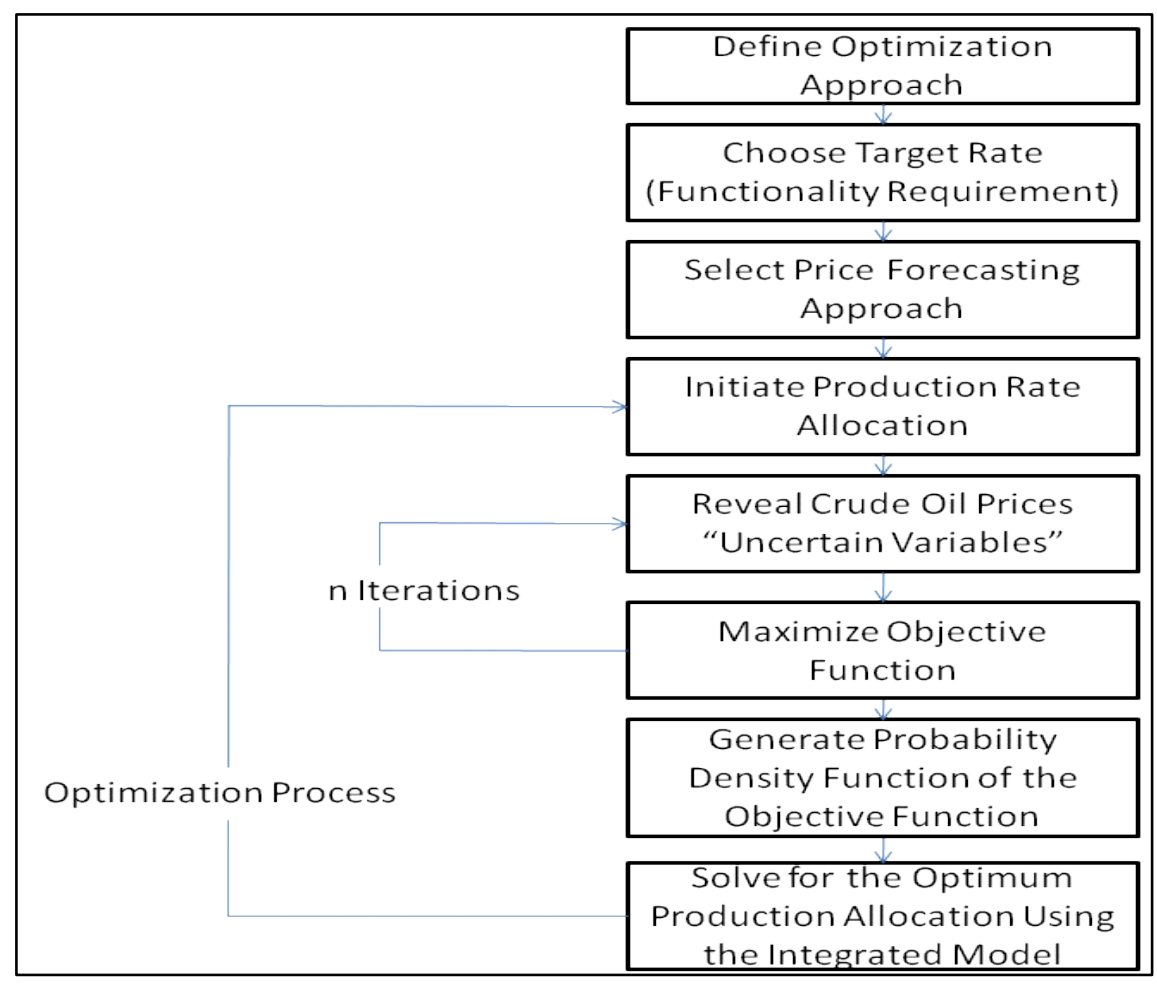

Figure 3.1: Risk Analysis Workflow

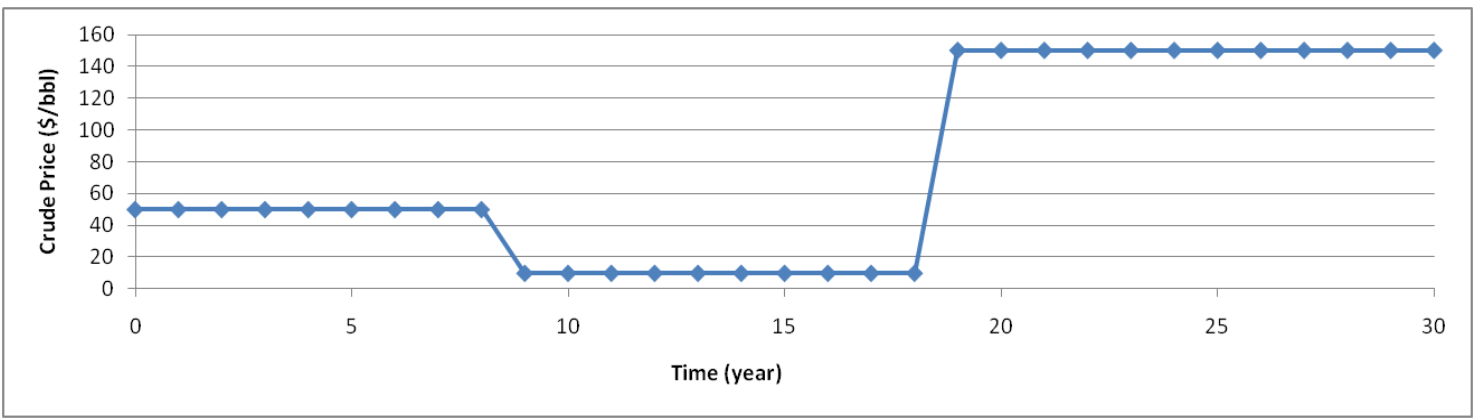

Figure 3.2: Hypothetical Price Forecast 


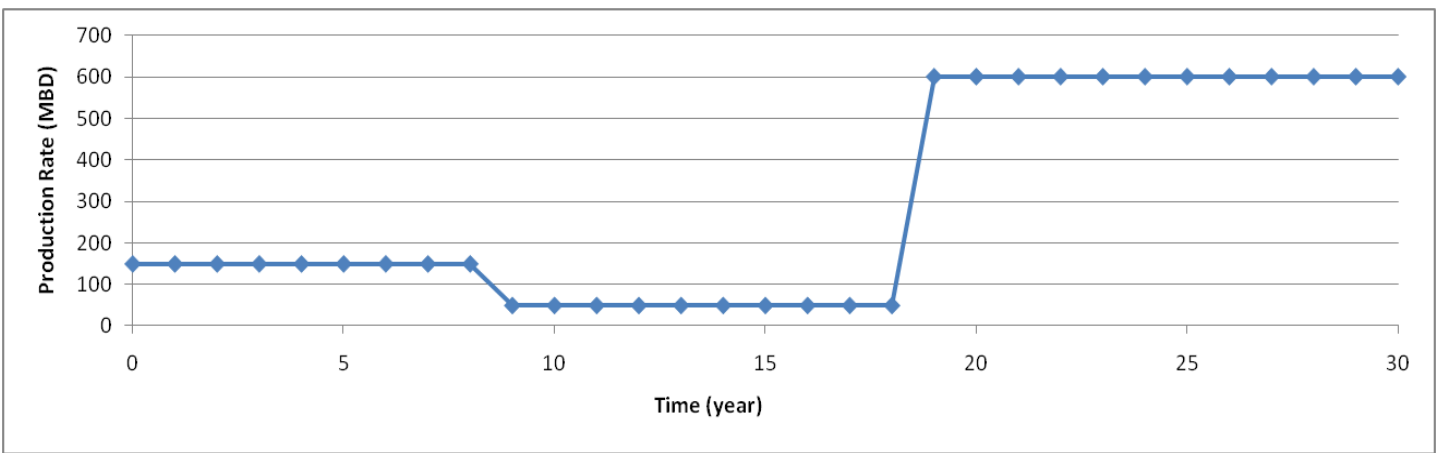

Figure 3.3: Production Allocation in Response to Figure 3.2 Price Forecast, which reveals the anticipatory error

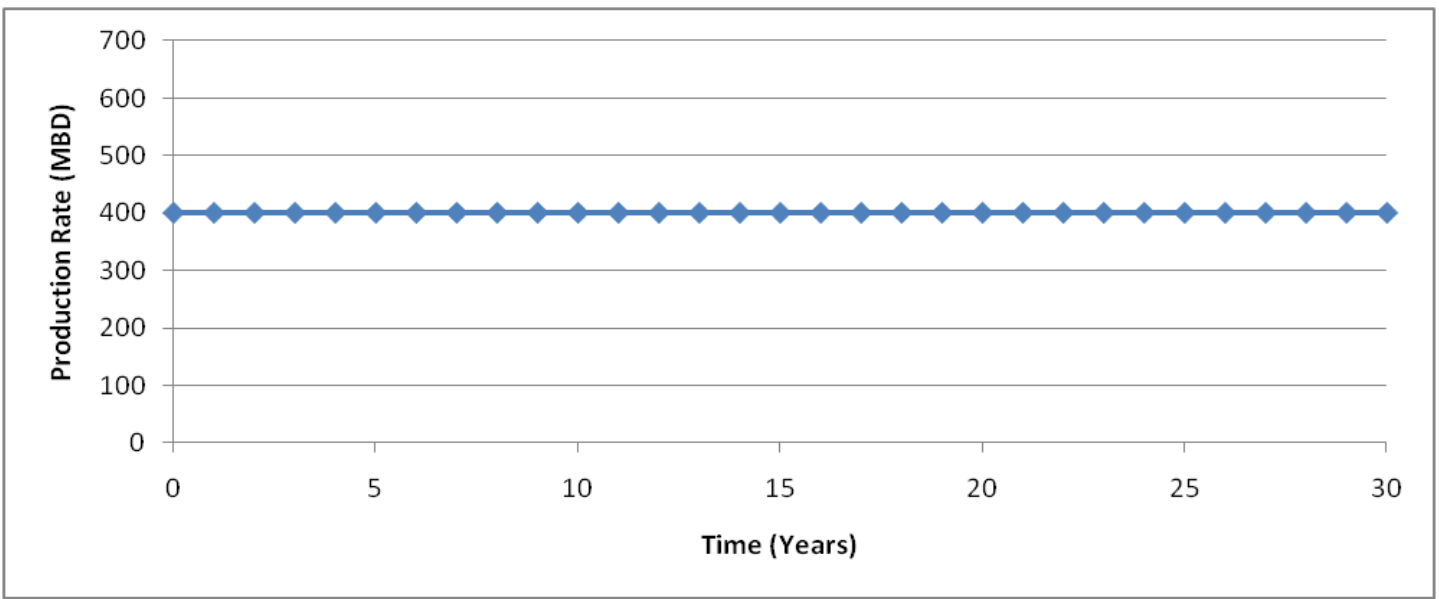

Figure 3.4: Optimum Production Allocation as a Single Value Eliminating the Anticipatory Error 


\section{CHAPTER 4: PRICE MODELS}

Crude oil prices had been stable at $\$ 3$ a barrel for 100 years from the birth of the oil industry in 1859 as illustrated in Figure 4.1. In 1973, oil prices jumped from $\$ 3$ to $\$$ 12 a barrel, for the first time, due to the Arab oil embargo. Then, crude oil prices had been stable or rising at stable rate for 10 years. In 1983, OPEC reduced oil prices, for the first time, from \$ 34 to \$29. In 1990, Gulf War raised the oil prices for only 6 months; then, the prices fell back. Toward the end of 2008, oil prices fell from around $\$ 140$ to $\$$ 70 within days. In summary, oil prices had been stable for the first 100 years of the oil industry then the cycle was reduced to 10 years, to 6 months and now the oil prices are volatile. Volatility increases the uncertainty involved in crude oil price forecast. Volatility and uncertainty have a significant impact on oil project decisions. In this chapter, crude oil prices are forecasted utilizing deterministic and probabilistic approaches. The deterministic approach is represented by Flat Price model and Floating

Price model, while the probabilistic approach is represented by Independent Lognormal Distribution (ILD), and stochastic processes (Geometric Brownian Motion (GBM), Mean Reversion Model (MRM), and Mean Reversion with Jumps (MRJM)). After building the crude oil price forecasting models, the price of different crudes are correlated. This chapter starts by estimating and calculating the needed parameters for crude oil price forecasting models. 


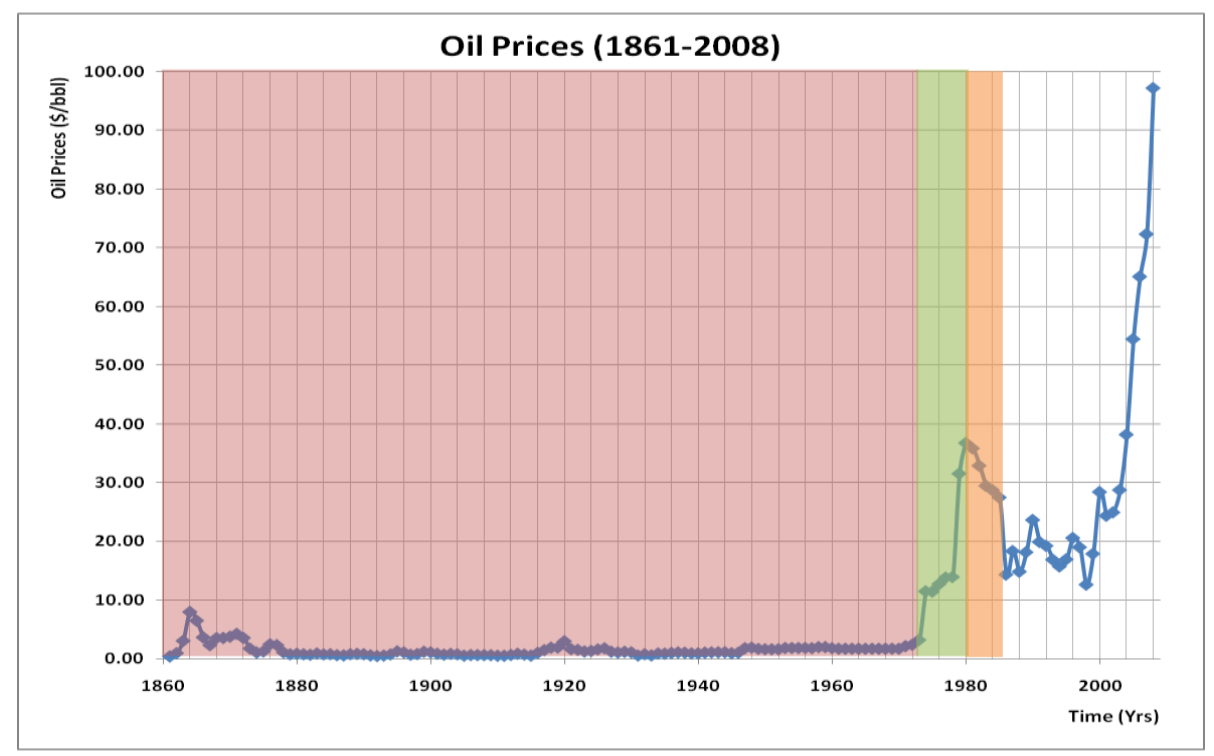

Figure 4.1: The Historical Data of Crude Oil Price

\subsection{Collecting Historical Price Data}

The research studies 23 fields that produce five different crude grades classified by API gravity as illustrated in Table 4.1 below.

Table 4.1: The Classifications of the Crudes

\begin{tabular}{|lcc|}
\hline \multicolumn{1}{|c}{ Crude Type } & Gravity ( ${ }^{\circ}$ API) & Producing Fields \\
\hline XX-Light Grade & $50-52$ & 3 \\
X-Light Grade & 39.5 & 4 \\
Light Grade & 34 & 8 \\
Medium Grade & 31 & 4 \\
Heavy Grade & 27 & 4 \\
\hline \hline \multicolumn{2}{|c|}{ Total } & 23 \\
\hline
\end{tabular}

The Light crude fields dominate the total production, which can be seen from the number of producing fields within each crude grade in Table 4.1. 
Crude price historical data is obtained for three crude grades (Heavy, Medium and Light) from the U.S. Energy Information Administration (www.eia.deo.gov). The weekly crude prices obtained covered the period between 1978 and 2010.

Using EXCEL functions Average and STDEV, the mean and standard deviation of the three available crude data sets were calculated. These properties are essential in forecasting crude prices probabilistically. The properties of the two missing crudes were calculated by assuming that these properties increase at a fixed rate per 1 API increase. Table 4.2 lists these probability distribution properties.

Table 4.2: $\quad$ Probability Distribution Properties of the Crudes

\begin{tabular}{|lccccccc|}
\hline \multicolumn{1}{|c}{ Crude Type } & Gravity ( ${ }^{\circ}$ API) & Distribution Type & Mean (\$) & Standard Deviation (\$) & Min (\$) & Max (\$) \\
\hline XX-Light & & & & & & & Remarks \\
X-Light & 52 & Lognormal & 44.06 & 88.94 & 10.56 & 148.00 \\
Light & 50 & Lognormal & 42.69 & 63.02 & 10.00 & 141.00 & Assumed \\
Medium & 34 & Lognormal & 34.41 & 44.89 & 9.65 & 136.02 & Historical Data \\
Heavy & 31 & Lognormal & 33.36 & 42.95 & 9.25 & 131.77 & Historical Data \\
\hline
\end{tabular}

\subsection{Programming Price Forecasting Models}

In this section, crude prices are forecasted using deterministic and probabilistic approaches. The deterministic approach is represented by the Flat Price and the Floating Price, while the probabilistic approach is represented by the Independent Lognormal Distribution and stochastic processes including the Geometric Brownian Motion, the Mean Reversion and the Mean Reversion with Jumps. The section starts by estimating and calculating the needed parameters for crude price forecasting whether deterministically or probabilistically.

\subsubsection{Crude Oil Price Modeling Parameters}

Price modeling processes, deterministic and probabilistic, require price modeling parameters. These parameters are critical in order to model "mean price equivalent" 
models. Crude price historical data is used to estimate the following parameters: crude mean price, annual price growth rate, standard deviation growth factor, price volatility, price drift, long run equilibrium price, price reversion rate, and price jump frequency and size.

\subsubsection{Crude Mean Price}

Mean crude price is a parameter that is critical to ensure that different crude price models are mean equivalent. Xu (2010) suggested that the history of mean crude price can be divided into three periods (Figure 4.2). Between 1987 and 2000, the mean price was around \$20; between 2000 and 2004, the mean price was around \$25; and from 2004 to the present, the mean price is around $\$ 80$. Xu (2010) suggested that $\$ 80$ per barrel is the new crude oil mean price or the long-run equilibrium price. As a result, historical data between 2004 and 2010 is acquired for crude mean price estimation.

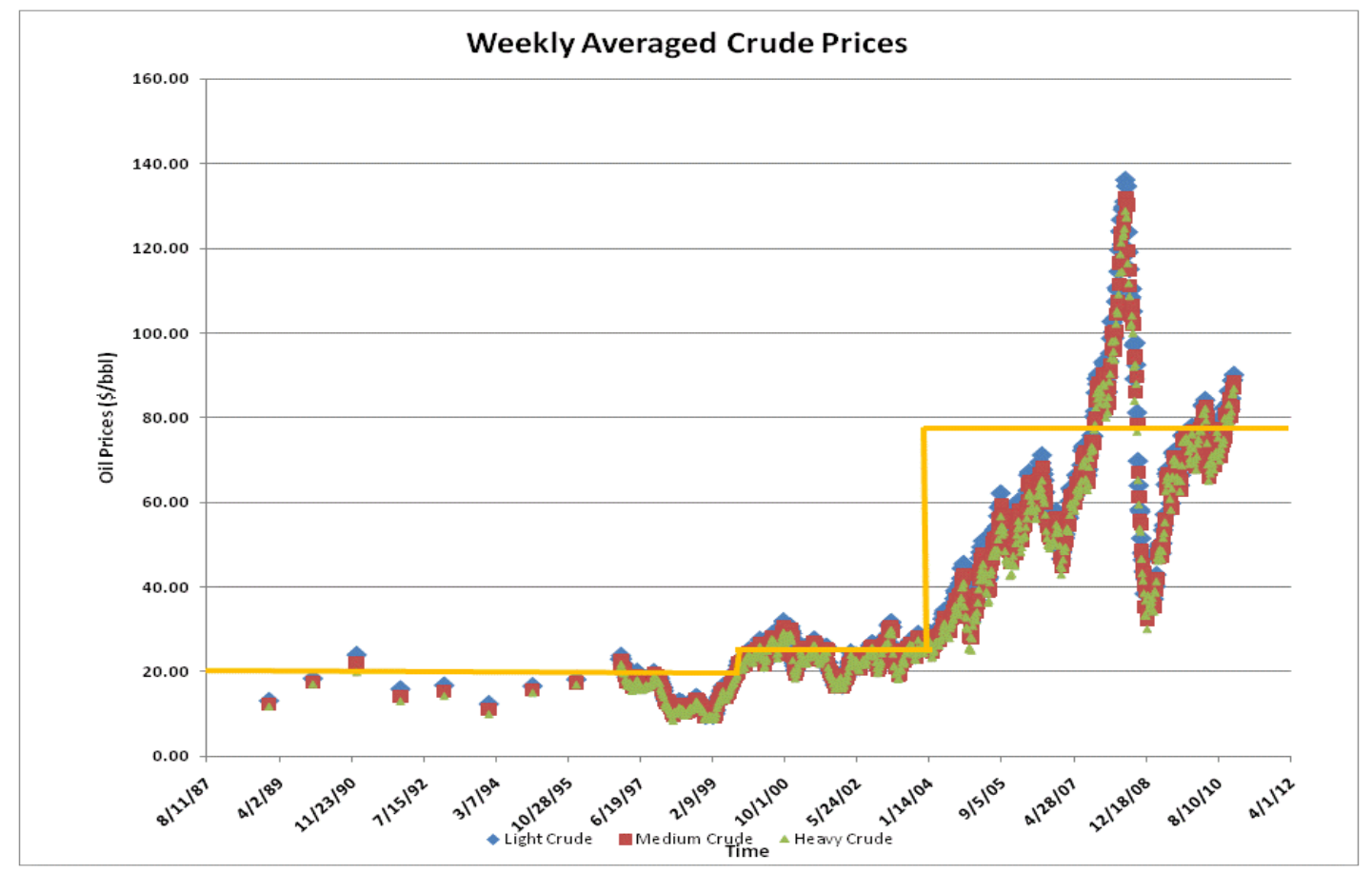

Figure 4.2: Different Long Run Equilibrium Crude Prices 1987-2012 
Price modeling parameters must be estimated using annual historical data because "annual" production rates and prices are used in the oil industry for economic evaluation and planning purposes. The acquired weekly crude price data was converted into annual data (Table 4.3), by deleting the additional data points. Then, the EXCEL functions, AVERAGE and STDEV, are used to estimate the mean and the standard deviation of the prices of the three available crudes, respectively. The mean prices and standard deviations for the two missing crudes were estimated using the method mentioned above, which assumes that mean and standard deviation increase at a fixed rate per 1 API increase in the crude grade.

Table 4.3: Annual Crude Prices (2004-2010)

\begin{tabular}{|cccc|}
\hline Date & $\begin{array}{c}\text { Light Crude Price } \\
\text { (\$ per Barrel) }\end{array}$ & $\begin{array}{c}\text { Medium Crude Price } \\
\text { (\$ per Barrel) }\end{array}$ & $\begin{array}{c}\text { Heavy Crude Price } \\
\text { (\$ per Barrel) }\end{array}$ \\
Jan 03, 2003 & 27.39 & 26.44 & 25.69 \\
Jan 02, 2004 & 27.08 & 26.13 & 25.38 \\
Jan 07, 2005 & 34.05 & 30.60 & 27.95 \\
Jan 06, 2006 & 55.01 & 52.86 & 50.41 \\
Jan 05, 2007 & 53.48 & 51.53 & 49.83 \\
Jan 04, 2008 & 93.02 & 90.27 & 88.07 \\
Jan 02, 2009 & 35.21 & 32.16 & 30.16 \\
Jan 01, 2010 & 75.21 & 74.01 & 73.56 \\
Dec 24, 2010 & 90.03 & 88.33 & 86.88 \\
\hline \hline Mean & 54.50 & 52.48 & 50.88 \\
S.D. & 26.11 & 26.08 & 26.06 \\
Min & 27.08 & 26.13 & 25.38 \\
Max & 93.02 & 90.27 & 88.07 \\
\hline
\end{tabular}




\subsubsection{The Rate of Crude Oil Price increases}

The assumption of a fixed crude price growth rate (Floating Price model) is a simple approach that is widely implemented in the oil industry. Price growth rate is calculated from historical annual price values between 2004 and 2010 as follows:

$$
\begin{aligned}
& F V=P V *(1+r)^{t} \\
& r=\left(\frac{F V}{P V}\right)^{\frac{1}{t}}-1
\end{aligned}
$$

Where

FV: Future value of crude price

PV: Present value of crude price

r: Annual price growth rate

t: Time in years

Equation 4.1 is the conventional formula for calculating future crude oil prices from current prices using growth rate $r$. Equation 4.2, derived from Equation 4.1, was used to calculate the annual price growth rate (r) between 2004 and 2010. The annual price growth rates used in the study are the average of the annual price growth rate for each crude grade. The average annual price growth factors are $0.25,0.23$ and 0.22 for the Light, Medium, and Heavy crudes, respectively.

\subsubsection{The Growth Rate of the Standard Deviation}

The growth rate of the standard deviation of the crude oil prices was used in the Independent LognNormal Distribution model (ILD) to reveal the increasing volatility and risk with time. To reflect the abnormal volatility of crude prices in the last seven years, historical crude price data between 2004 and 2010 were used in the calculations. Faya (2006) implemented Equation 4.3 to calculate the future standard deviation of crude oil

prices from the current ones. Rearranging Equation 4.3, Equation 4.4 calculates the 
annual standard deviation growth factor. The calculation of the standard deviation growth rate is conducted in three steps. First, the standard deviations of every two consecutive annual prices in the historical data are calculated using the STDEV function in EXCEL. Then, using Equation 4.4, the annual growth rates of the standard deviations are calculated for every two entries of standard deviation. The last step is to average the standard deviation annual growth rates calculated; the average annual standard deviation growth rates used in the study are 4.79, 4.60, and 4.39 for the Light, Medium, and Heavy crudes respectively.

$$
\begin{aligned}
& S D(t)=S D(0)+G F * t \\
& G F=\left|\frac{S D(t)-S D(0)}{t}\right|
\end{aligned}
$$

Where

$\mathrm{SD}(\mathrm{t})$ : crude price standard deviation for a given year " $\mathrm{t}$ "

$\mathrm{SD}(0)$ : crude price standard deviation for the present year

GF: standard deviation growth factor

$\mathrm{t}$ : time in years

\subsubsection{Crude Oil Price Volatility (б)}

Volatility is the standard deviation of logarithm returns, and is the term of uncertainty in stochastic/probabilistic price models, which are Geometric Brownian Motion, Mean Reversion and Mean Reversion with Jumps models. Table 4.4 shows the volatility calculations for the Light crude price data from annual historical data between 2004 and 2010. Annual crude oil prices are listed in the second column. In the third the fourth column, the natural logs of crude prices of the current period and previous period are listed, respectively. The volatility is the standard deviation of all the entries in the fifth column, which is the difference between the third and fourth columns entries, as 
described by Equation 4.5. The volatility is similarly calculated for the other crude grades. However, different sets of crude historical data are used to calculate volatility for different stochastic processes. For example, annual price data between 2004 and 2010 is used to calculate volatility for the Mean Reversion model. Weekly price data between 1978 and 2010 is used to calculate volatility for the Geometric Brownian Motion model, then the weekly value is converted to annual value by multiplying weekly volatility by $\sqrt{52}$. For the Mean Reversion with Jumps model, weekly price data between 2004 and 2010 is used to calculate the volatility since the Mean Reversion with Jumps model forecasts weekly prices as illustrated in the Mean Reversion with Jumps modeling section. Table 4.5 summarizes the volatility values used in each stochastic process and the price data set used to calculate each value.

The discrepancy in the volatility values in Table 4.5 is due to the nature of each stochastic process. The GBM is a diffusion of a lognormal distribution as illustrated in the GBM modeling section which means the higher the volatility, the higher the range of possible prices. More accurate and reasonable volatility is required for this type of process to obtain a reasonable range of crude oil price forecasts. That is the reason for using all the available price data (1987 to 2010 weekly data) to calculate the volatility of the GBM model. Since the data used to calculate the GBM volatility is a weekly data set, the value is converted to annual data by multiplying the value by $\sqrt{52}$. For the mean reversion model (MRM), the forecasting process assumes that the crude oil price reverts to a long run equilibrium price with a given volatility, so the price range does not expand with time. As a result, it is critical to incorporate the high crude price volatility of the last seven years. The MRM volatility is calculated based on 2004 to 2010 annual price data. Moreover, 2004 is the beginning of the new mean crude price as suggested by $\mathrm{Xu}(2010)$. Unlike the GBM and the MRM, the accuracy of the Mean Reversion with Jumps model 
depends on the size of time step $(\Delta t)$ used, which is discussed in detail in the MRM with Jump modeling section. The smaller the time step, the more accurate the model is. Consequently, weekly (small time step) crude price forecasting is implemented in the MRM with Jumps model. Using weekly forecasts reduced the time step from one year to $7 / 365$ or 0.019178 year. At the same time, the MRM with Jumps follows the basic concept of reverting to long price equilibrium, so the high volatility of crude oil prices should be incorporated into the modeling process, which can be obtained from crude historical data between 2004 and 2010. The MRM with Jumps Volatility is calculated from weekly data between 2004 and 2010.

$$
\sigma=\text { Standard Deviation }[\ln P(t)-\ln P(t-1)], \forall t
$$

Table 4.4: The Light Crude Volatility Calculations Using Annual Historical Data

\begin{tabular}{|lcccc|}
\hline Time (Yrs) & $\begin{array}{c}\text { Light Crude } \\
\text { Price }\end{array}$ & Ln[P(t)] & Ln[P(t-1)] & Ln[P(t)]-Ln[P(t-1)] \\
& & & & \\
Jan 03, 2003 & 27.39 & & & \\
Jan 02, 2004 & 27.08 & 3.30 & 3.31 & -0.01 \\
Jan 07, 2005 & 34.05 & 3.53 & 3.30 & 0.23 \\
Jan 06, 2006 & 55.01 & 4.01 & 3.53 & 0.48 \\
Jan 05, 2007 & 53.48 & 3.98 & 4.01 & -0.03 \\
Jan 04, 2008 & 93.02 & 4.53 & 3.98 & 0.55 \\
Jan 02, 2009 & 35.21 & 3.56 & 4.53 & -0.97 \\
Jan 01, 2010 & 75.21 & 4.32 & 3.56 & 0.76 \\
Dec 24, 2010 & 90.03 & 4.50 & 4.32 & 0.18 \\
\hline $\boldsymbol{\sigma}$ & \multicolumn{5}{c}{$\mathbf{0 . 5 3}$} \\
\hline
\end{tabular}


Table 4.5: Volatility Values for Different Stochastic Processes

Stochastic Process Volatility $(\sigma)$ Geometric Brownian Motion

Mean Reversion

Mean Reversion with Jumps
$42.26 \%$

$59.37 \%$

$4.90 \%$
Data Set Used

(1978-2010) Weekly Converted to Annual

(2004-2010) Annual

(2004-2010) Weekly

\subsubsection{Crude Oil Price Drift ( $\alpha)$}

As mentioned earlier, the Geometric Brownian Motion model (GBM) is a diffusion of lognormal distribution with time. Drift is the trend or the linear growth rate of the diffusion process. Table 4.6 shows the drift calculations of the Light crude from annual historical data between 2004 and 2010. The setup of Table 4.6 is similar to Table 4.4; the only difference is the two entries at the bottom of Table 4.6, $\alpha-\frac{1}{2} \sigma^{2}$ and $\alpha$. The first term $\alpha-\frac{1}{2} \sigma^{2}$, is the average of logarithm returns, which can be calculated using Equation 4.6. $\alpha-\frac{1}{2} \sigma^{2}$ is the average of all the entries in the fifth column in Table-6. After calculating the first term $\alpha-\frac{1}{2} \sigma^{2}$, the drift is calculated by adding $\frac{1}{2} \sigma^{2}$ to that term as shown in Equation-4.7. The drift values used in the study are 0.289, 0.311, and 0.329 for the Light, Medium, and Heavy crudes, respectively.

Table 4.6: The Light Crude Drift Calculations Using Annual Historical Data 


\begin{tabular}{|c|c|c|c|c|}
\hline \multirow[b]{2}{*}{ Time (Yrs) } & \multicolumn{4}{|c|}{ Light Crude } \\
\hline & $\begin{array}{l}\text { Light Crude } \\
\text { Price }(\$ / \mathbf{b b l})\end{array}$ & $\operatorname{Ln}[P(t)]$ & $\operatorname{Ln}[P(t-1)]$ & $\operatorname{Ln}[P(t)]-\operatorname{Ln}[P(t-1)]$ \\
\hline Jan 03, 2003 & 27.39 & & & \\
\hline Jan 02, 2004 & 27.08 & 3.30 & 3.31 & -0.01 \\
\hline Jan 07, 2005 & 34.05 & 3.53 & 3.30 & 0.23 \\
\hline Jan 06, 2006 & 55.01 & 4.01 & 3.53 & 0.48 \\
\hline Jan 05, 2007 & 53.48 & 3.98 & 4.01 & -0.03 \\
\hline Jan 04, 2008 & 93.02 & 4.53 & 3.98 & 0.55 \\
\hline Jan 02, 2009 & 35.21 & 3.56 & 4.53 & -0.97 \\
\hline Jan 01, 2010 & 75.21 & 4.32 & 3.56 & 0.76 \\
\hline \multirow[t]{3}{*}{ Dec 24, 2010} & 90.03 & 4.50 & 4.32 & 0.18 \\
\hline & & $\left.1 / 2 \sigma^{2}\right)$ & & 0.149 \\
\hline & & & & 0.289 \\
\hline
\end{tabular}

$$
\begin{aligned}
& \left(\alpha-\frac{1}{2} \sigma^{2}\right)=\text { Average }[\ln P(t)-\ln P(t-1)], \forall t \\
& \alpha=\left(\alpha-\frac{1}{2} \sigma^{2}\right)+\left(\frac{1}{2} \sigma^{2}\right)
\end{aligned}
$$

\subsubsection{Long-run Equilibrium Price $\left(P^{*}\right)$ and Price Reversion Rate ( $\eta$ )}

Long-run equilibrium price and price reversion rate are estimated from the same regression analysis, which means estimating one of them results in the other. In other words, the calculations of the two parameters are compliments; hence they are combined in one section.

The long equilibrium price $\left(\mathrm{P}^{*}\right)$ is the mean price that prices tend to revert to over time. Reversion rate $(\eta)$ is the speed at which prices revert to the long-run mean price. These two parameters are needed for the GBM, the MRM and the MRM with Jumps models. The two parameters are estimated from annual historical price data between 2004 and 2010, as suggested by $\mathrm{Xu}$ (2010), for the GBM and the MRM price models. On the other hand, weekly price data between 2004 and 2010 are used to estimate $\mathrm{P}^{*}$ and $\eta$ for the MRM with Jumps model because the model forecasts weekly prices.

The two parameters are estimated using the first-order autoregressive process, AR(1), in discrete time as suggested by Dixit and Pindyck (1994). The AR(1) process is 
expressed in Equation 4.8. Substituting equations 4.9 and 4.10 into 4.8 results in Equation 4.11, which is a linear regression with slope $\mathrm{b}$, intersection $a$, and residual $\varepsilon_{\mathrm{t}}$. In regression analysis, the residual is a normal distribution with mean zero and standard deviation equal to the standard error $\left(\sigma_{\mathrm{E}}\right)$ of the regression data. The residual $\left(\varepsilon_{\mathrm{t}}\right)$ is the difference between the actual and simulated data or the error term.

Table 4.7 shows regression analysis data used in $\mathrm{P}^{*}$ and $\eta$ estimation. The fourth column $(\mathrm{P}(\mathrm{t})-\mathrm{P}(\mathrm{t}-1))$ is the dependent variable and the third column $(\mathrm{P}(\mathrm{t}-1))$ represents the independent variable of the regression analysis. Figure 4.3 is the regression analysis plot with regression parameters, shown in Table 4.8 , which are used to estimate $\mathrm{P}^{*}$ and $\eta$ for the Heavy crude. Using equations 4.14 and $4.15, \mathrm{P}^{*}$ and $\eta$ are estimated with values 56.23 and 1.50 , respectively.

$$
\begin{aligned}
& P_{(t)}-P_{(t-1)}=P^{*} *\left(1-e^{-\eta}\right)+\left(e^{-\eta}-1\right) * P_{(t-1)}+\varepsilon_{t} \\
& P^{*}\left(1-e^{-\eta}\right)=a \\
& \left(e^{-\eta}-1\right)=b \\
& P_{(t)}-P_{(t-1)}=a+b * P_{(t-1)}+\varepsilon_{t} \\
& \varepsilon_{t}=N\left(0, \sigma_{c}\right) \\
& \sigma_{E}=\text { Standard Error } \\
& P^{*}=-\frac{a}{b} \\
& \eta=-\ln (1+b)
\end{aligned}
$$

Table 4.7: The Heavy Crude Regression Analysis for $P^{*}$ and $\eta$ Estimation 


\begin{tabular}{|c|c|c|c|}
\hline \multicolumn{4}{|c|}{ Historical Data (Light) } \\
\hline Date & $\mathbf{P}(\mathbf{t})$ & $P(t-1)$ & $P(t)-P(t-1)$ \\
\hline & & X-Variable & Y-Variable \\
\hline Jan 02, 2004 & 25.38 & 25.69 & -0.31 \\
\hline Jan 07, 2005 & 27.95 & 25.38 & 2.57 \\
\hline Jan 06, 2006 & 50.41 & 27.95 & 22.46 \\
\hline Jan 05, 2007 & 49.83 & 50.41 & -0.58 \\
\hline Jan 04, 2008 & 88.07 & 49.83 & 38.24 \\
\hline Jan 02, 2009 & 30.16 & 88.07 & -57.91 \\
\hline Jan 01, 2010 & 73.56 & 30.16 & 43.40 \\
\hline Dec 24, 2010 & 86.88 & 73.56 & 13.32 \\
\hline
\end{tabular}

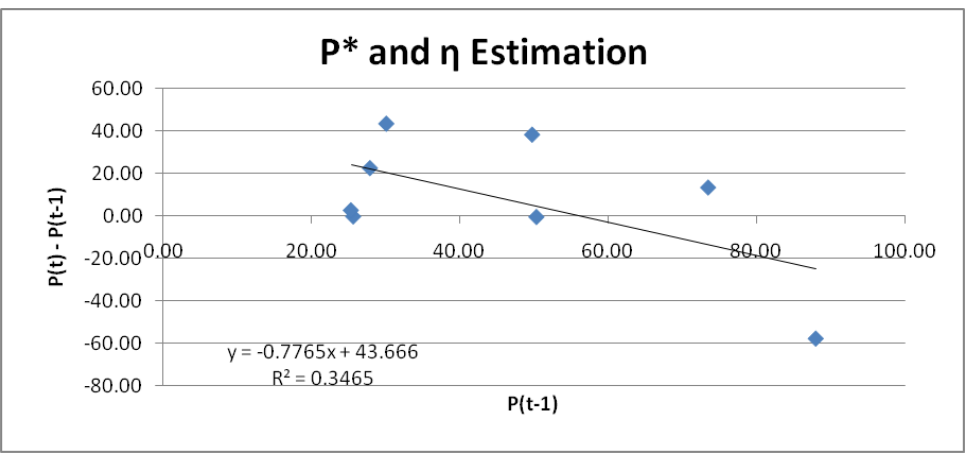

Figure 4.3: $\quad \mathrm{P}^{*}$ and $\eta$ Estimation Using Regression Analysis (AR(1))

Table 4.8: $\quad$ Regression Analysis (AR(1)) Parameters

\begin{tabular}{|lrl|}
\hline \multicolumn{3}{|c|}{ Regression Parameters } \\
a & 43.67 & Y-Intercept \\
$\mathbf{b}$ & -0.78 & Slope \\
$\boldsymbol{\sigma}_{\boldsymbol{\varepsilon}}$ & 27.45 & Standard Error \\
\hline
\end{tabular}

\subsubsection{Jump Size $(\phi)$ and Frequency $(\lambda)$}

The Mean Reversion with Jumps model correlates price changes to new arrivals. Jumps represent the arrival of abnormal political or environmental information, which has a significant impact on crude prices (Dias, 2010a). The impact severity of information arrival on crude prices is represented by jump size $(\varphi)$, and the frequency of arrival of that type of information is represented by jump frequency $(\lambda)$. The greater the information's impact on prices, the greater the jump size to model the information arrival. Jump frequency is the arrival rate of abnormal information (jumps); for example, $\lambda=$ 
0.25 , means that one abnormal information (jump) arrives every four time periods. Price jump size and frequency are only needed for the MRM with Jumps price model. Moreover, an interesting property of jumps is that they are independent of historical data since jumps depend on abnormal information arrival. As a result, it is critical to estimate these two parameters, and it is better to choose reasonable parameters than to calculate non-reasonable ones from historical data as suggested by Dias (2010a). The values of $\left(\frac{1}{8}\right)$ and $\ln (2)$ for jump frequency and size respectively are suggested by Dr. Dias and are used for this study.

\subsection{Crude Oil Price Modeling}

\subsubsection{Deterministic Forecasting Models}

The deterministic approach, which assumes crude forecasting parameters are known and fixed, is a simple and widely implemented approach. In other words, this approach neglects crude price uncertainty. The deterministic approach is incorporated into the study by programming two price forecasting models, the Flat Price Model and the Floating Price Model. This section describes in detail the procedure for programming these models.

\subsubsection{The Flat Price Model}

The Flat Price is the simplest price forecasting model. The model assumes a single value for each crude grade throughout the life of the project. The long-run mean price $\left(\mathrm{P}^{*}\right)$, which is calculated for stochastic processes, is the crude price used for this model. Table 4.9 and Figure 4.4 show the Flat Price model.

Table 4.9: The Flat Price Model 


\begin{tabular}{|lcc|}
\hline \multicolumn{1}{|c}{ Crude } & Mean Price (\$) & Remarks \\
Heavy & 56.23 & Calculated \\
Medium & 57.99 & Calculated \\
Light & 60.36 & Calculated \\
X-Light & 62.16 & Assumed \\
XX-Light & 64.14 & Assumed \\
\hline
\end{tabular}

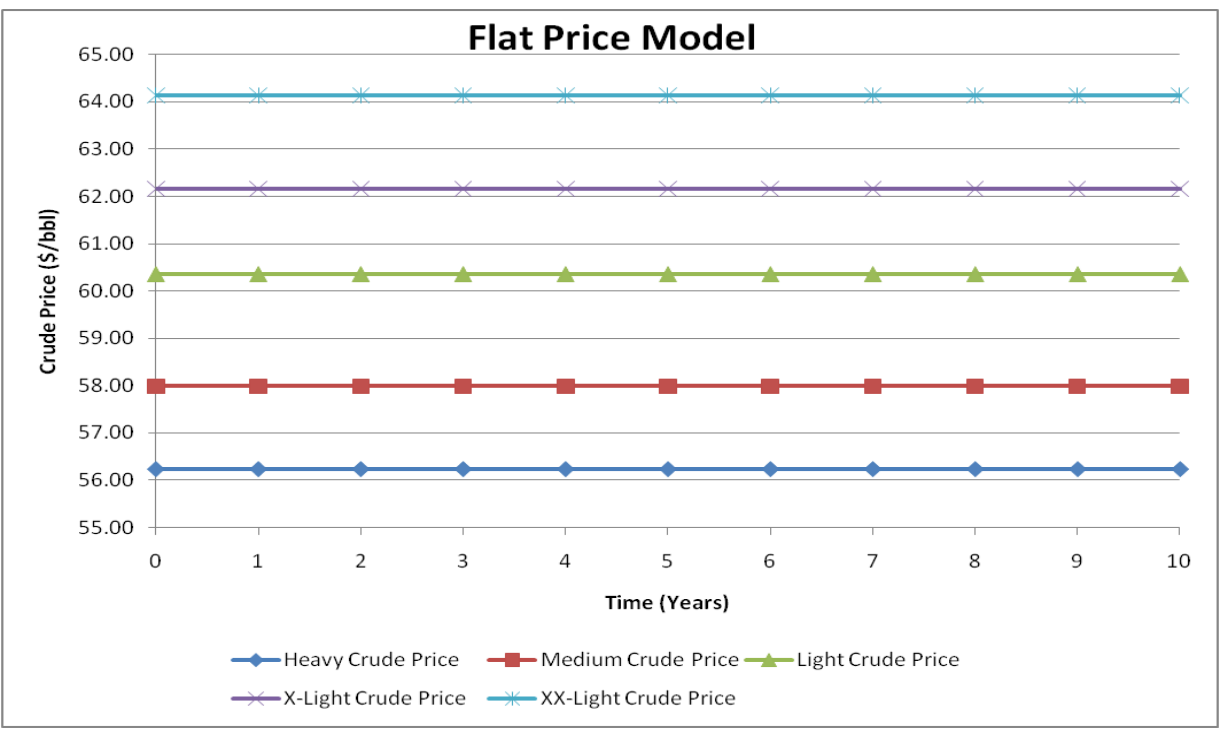

Figure 4.4: The Flat Price Model

\subsubsection{The Floating Price Model}

A fixed growth rate model is widely used in the oil industry. This type of model assumes that crude prices are "inelastic" which means that crude prices grow with time neglecting the effect of demand. The model starts with a given crude price for each crude grade. Then, imposing the annual crude price growth rate, calculated in the parameter section, crude prices are forecasted; Equation 4.16 is used to forecast future prices. The initial prices used in the study are the long-run mean prices $\left(\mathrm{P}^{*}\right)$, so that forecast models are mean equivalent. Table 4.10 shows the initial prices and growth rates for each crude grade used in this model. Table 4.11 and Figure 4.5 show the price forecasts of the Floating Price model. 


$$
F V=P V *(1+r)^{t}
$$

Table 4.10: Initial Crude Prices and Annual Growth Rates Used in the Floating Price Model

\begin{tabular}{|lccl|}
\hline \multicolumn{1}{|c}{ Crude } & Mean Price (\$) & Growth Rate & Remarks \\
Heavy & 56.23 & 0.22 & Calculated \\
Medium & 57.99 & 0.23 & Calculated \\
Light & 60.36 & 0.25 & Calculated \\
X-Light & 62.16 & 0.27 & Assumed \\
XX-Light & 64.14 & 0.30 & Assumed \\
\hline
\end{tabular}

Table 4.11: The Floating Price Model

\begin{tabular}{|lccccccccccc|}
\hline Time & $\mathbf{0}$ & $\mathbf{1}$ & $\mathbf{2}$ & $\mathbf{3}$ & $\mathbf{4}$ & $\mathbf{5}$ & $\mathbf{6}$ & $\mathbf{7}$ & $\mathbf{8}$ & $\mathbf{9}$ & $\mathbf{1 0}$ \\
Heavy Crude Price & 56.23 & 68.68 & 83.88 & 102.44 & 125.10 & 152.79 & 186.59 & 227.89 & 278.31 & 339.90 & 415.11 \\
Medium Crude Price & 57.99 & 71.51 & 88.19 & 108.76 & 134.13 & 165.41 & 203.99 & 251.57 & 310.24 & 382.60 & 471.84 \\
Light Crude Price & 60.36 & 75.24 & 93.78 & 116.90 & 145.71 & 181.62 & 226.39 & 282.19 & 351.75 & 438.45 & 546.52 \\
X-Light Crude Price & 62.16 & 78.94 & 100.25 & 127.32 & 161.70 & 205.36 & 260.81 & 331.23 & 420.66 & 534.23 & 678.48 \\
\hline XX-Light Crude Price & 64.14 & 83.38 & 108.39 & 140.91 & 183.18 & 238.14 & 309.58 & 402.45 & 523.19 & 680.14 & 884.19 \\
\hline
\end{tabular}

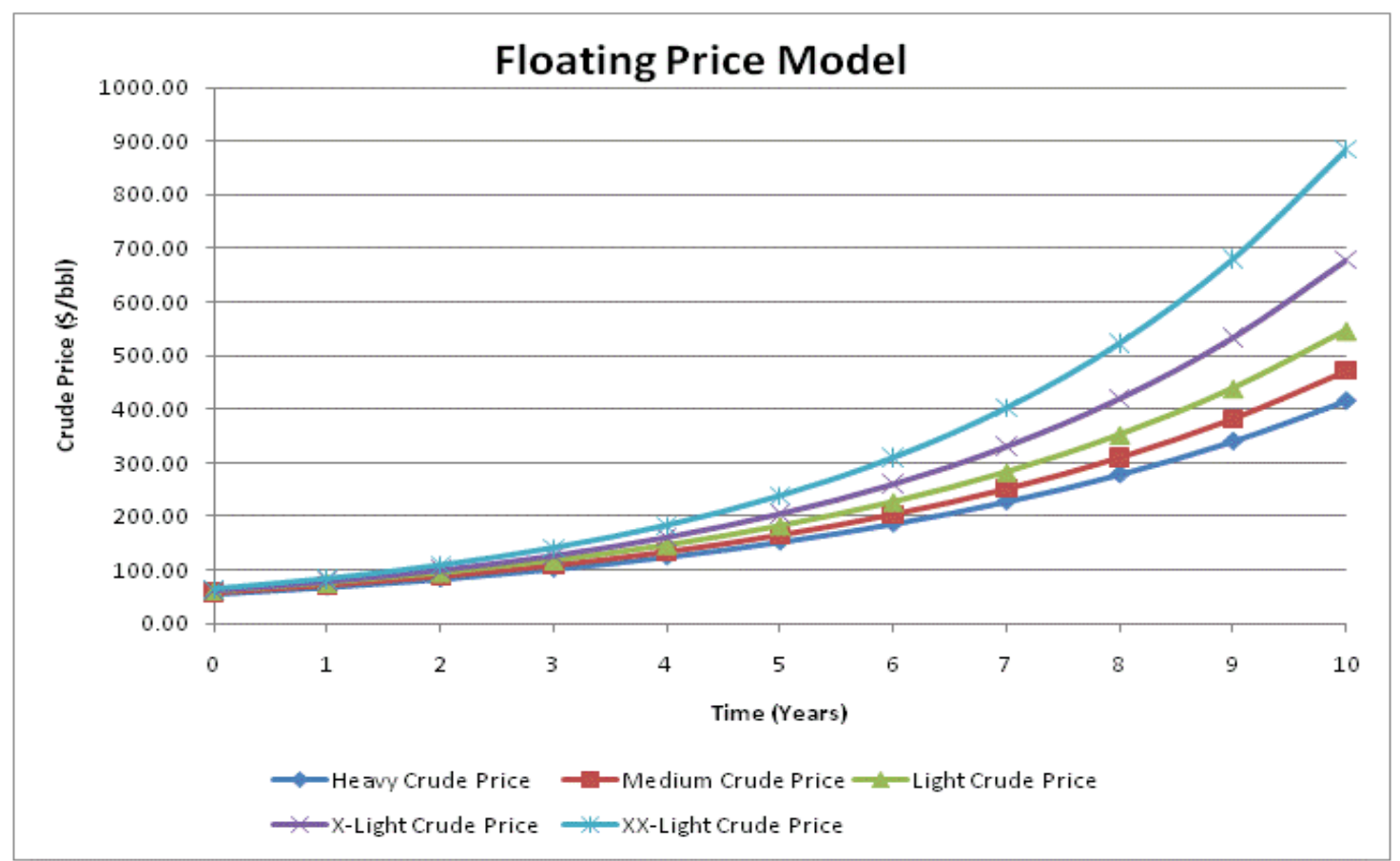

Figure 4.5: The Floating Price Model 


\subsubsection{Probabilistic Forecasting Models}

Crude price volatility encouraged the oil industry to conduct more research in the area of Risk and Decision Analysis. Price modeling might affect decision making during project planning stages. In this section, four commonly used stochastic forecasting processes are introduced, and then modeling processes are discussed. The four price forecasting processes are Independent Lognormal Distribution (ILD), Geometric Brownian Motion (GBM), Mean Reversion Model (MRM) and Mean Reversion with Jumps (MRM with Jumps). Risk Solver Platform (RSP), Microsoft EXCEL add-in, is used in the forecasting process. RSP is a powerful tool that performs optimization (linear, non-linear programming), simulation, and regression at the same time. Moreover, RSP generates probability distribution by specifying distribution properties including distribution type, mean and standard deviation. RSP is a reliable and fast solving algorithm which facilitates the achievement the study's objectives. In other words, RSP facilitates fast solving and generating results of many parameter realizations within a specific price forecasting model; as a result, more and more scenarios can be generated and solved to compare, contrast and evaluate the impact of the price forecasting models' parameters on the allocation decision. Moreover, the speed of the RSP is effective in sensitivity analysis of the price modeling parameters.

\subsubsection{The Independent Lognormal Distribution (ILD)}

The ILD model is the simplest form of a price forecasting stochastic process. This process assumes that crude prices are log normally distributed during each time period, and that this distribution is independent of time. We modified the ILD process to include the increasing volatility with time effect in the price modeling process. The assumption that crude price volatility increases with time is imposed on the ILD model by relating the standard deviation of the lognormal distribution with time. In summary, the ILD 
model is a lognormal distribution model with a fixed mean price and an increasing standard deviation with time.

Long-run mean prices $\left(\mathrm{P}^{*}\right)$ are used as the mean prices for the lognormal price distribution to ensure mean-equivalent forecasting models. Table-12 shows the mean prices and initial standard deviations used in the ILD model; the two parameters are estimated from 2004-2010 annual historical data. The initial standard deviation is the standard deviation used in the first year of price forecasting. Table-13 shows the standard deviation growth factor, estimated from 2004-2010 annual historical data, and the minimum Heavy crude price. The Heavy crude minimum price is a floor price that the Heavy crude price cannot drop below. We believe that the Heavy crude price cannot fall below $\$ 40$ with the new mean price around $\$ 80$. Future standard deviation values were calculated using Equation 4.17. Using Equation 4.17, the Heavy crude standard deviation values were calculated; the Heavy standard deviations for the first five periods are presented in Table 4.14. The Heavy crude prices are forecasted as shown in Table-15 using Equation 4.18. Equation-Z.18 states that the Heavy crude price at given time $t$ is the maximum value between the lognormal distribution, with known mean price and standard deviation at $\mathrm{t}$, and the Heavy crude minimum price. Figure 4.6 shows the Heavy crude forecasted prices; the figure shows the increasing volatility (standard deviation) with time. The other crude prices are forecasted using a correlation technique where the Medium, the Light, the X-Light and the XX-Light crudes are correlated to the Heavy crude prices. The correlation process is discussed in the Crude Price Correlation section. 
Table 4.12: Crude Mean Prices and Initial Standard Deviations

\begin{tabular}{|lcc|}
\hline Crude & Mean Price (\$) & Initial S.D. \\
Heavy & 56.23 & 26.06 \\
Medium & 57.99 & 26.08 \\
Light & 60.36 & 26.11 \\
X-Light & 62.16 & 26.33 \\
XX-Light & 64.14 & 26.64 \\
\hline
\end{tabular}

Table 4.13: The Growth Rate of the Heavy Price Standard Deviation, and the Heavy Crude Minimum Price

\begin{tabular}{|lc|}
\hline S.D. GF & 4.39 \\
P(min), heavy & 40.00 \\
\hline
\end{tabular}

Table 4.14: The Heavy Crude Standard Deviation

\begin{tabular}{|lcccccc|}
\hline Time (Years) & 0 & 1 & 2 & 3 & 4 & 5 \\
Heavy S.D. & 26.06 & 30.45 & 34.85 & 39.24 & 43.63 & 48.02 \\
\hline
\end{tabular}

$S D(t)=S D(0)+G F * t$

Where

$\mathrm{SD}(\mathrm{t})$ : crude price standard deviation at given year $\mathrm{t}$

$\mathrm{SD}(0)$ : crude price standard deviation for the present year

GF: standard deviation growth factor

$\mathrm{t}$ : time in years

$P(t)_{\text {Heavy }}=\operatorname{MAX}\left[\right.$ PsiLognormal $($ MeanPrice, $\left.S D(t)), P(M I N)_{\text {Heavy }}\right]$

Table 4.15: The ILD Price Forecast Model

\begin{tabular}{|lllllll|}
\hline Heavy Crude Price & 50.87 & 134.30 & 43.62 & 53.35 & 47.82 & 115.80 \\
Medium Crude Price & 51.30 & 136.80 & 45.55 & 54.53 & 49.38 & 117.88 \\
Light Crude Price & 52.88 & 139.36 & 45.66 & 54.74 & 51.93 & 120.64 \\
X-Light Crude Price & 55.19 & 141.95 & 47.73 & 61.36 & 50.08 & 123.96 \\
XX-Light Crude Price & 56.73 & 145.81 & 49.42 & 61.28 & 54.56 & 133.65 \\
\hline
\end{tabular}




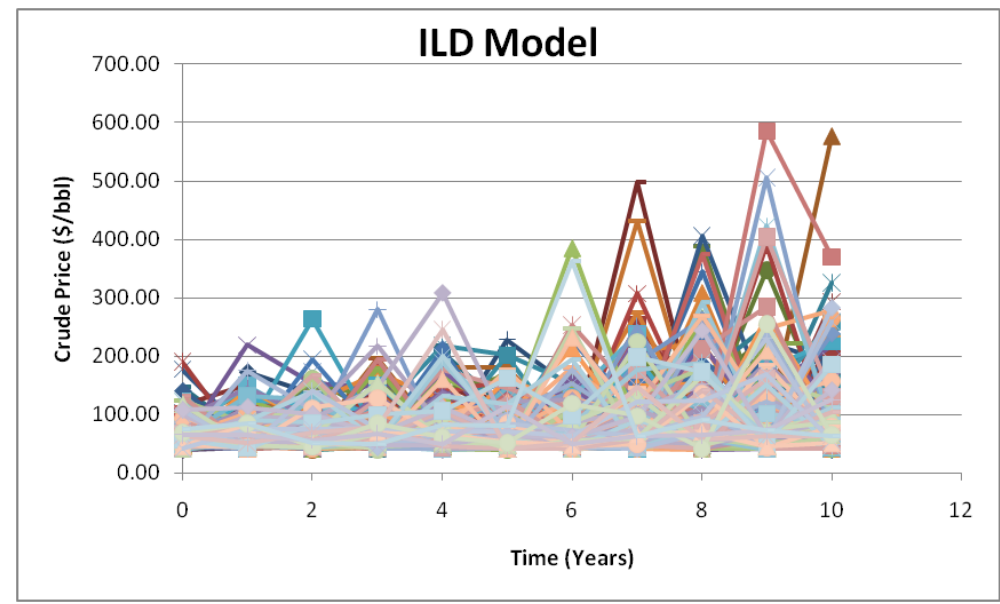

Figure 4.6: Independent Lognormal Distribution Forecast Model

\subsubsection{The Geometric Brownian Motion (GBM)}

\subsection{The GBM Model, Background}

Brownian motion, known as the Wiener process, is a special version of the Markov process. Brownian motion is a stochastic process with zero mean and variance of one per time period. The process was first introduced by Robert Brown in 1827 to describe the motion of small particles subject to a large number of small random collisions in physics. The Wiener process is stationary; it does not incorporate growth rate with time (drift) (Dyer, 2011). By adding a growth rate to the Wiener process, Arithmetic Brownian Motion (ABM) is obtained. In other words, ABM is a combination of a linear growth rate (drift) and normally distributed random growth (Dyer, 2011). Equation 4.19 and Figure 4.7 show the ABM formula and sample, respectively. A more appropriate process to forecast price is known as the Geometric Brownian Motion (GBM) where drift and proportional volatility are constant. The GBM is a combination of proportional growth rate (drift) and a normally distributed random proportional growth. Equation 4.20 and Figure 4.8 show the GBM formula in continuous form and the GBM 
sample, respectively. The Geometric Brownian Motion is a log-normal diffusion process, where variance grows with time as shown in Figure 4.9 (Dias, 2010b).

$$
\begin{aligned}
& P(t+1)=P(t)+\alpha * d t+\sigma * d z \\
& d P=\alpha * P * d t+\sigma * P * d z
\end{aligned}
$$

Where

$$
\begin{aligned}
& \mathrm{dz}(\text { Wiener increment })=\varepsilon \mathrm{dt}^{1 / 2} \\
& \varepsilon=\text { Standard Normal }=\mathrm{N}(0,1) \\
& \alpha=\text { Drift or trend } \\
& \sigma=\text { Volatility of } \mathrm{P}
\end{aligned}
$$

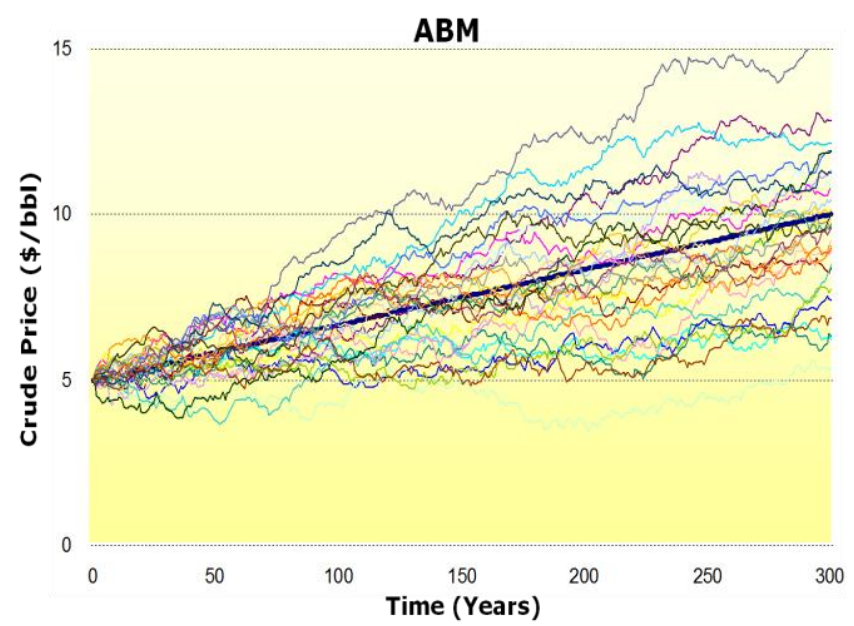

Figure 4.7: Arithmetic Brownian Motion Samples (Dyer, 2011) 


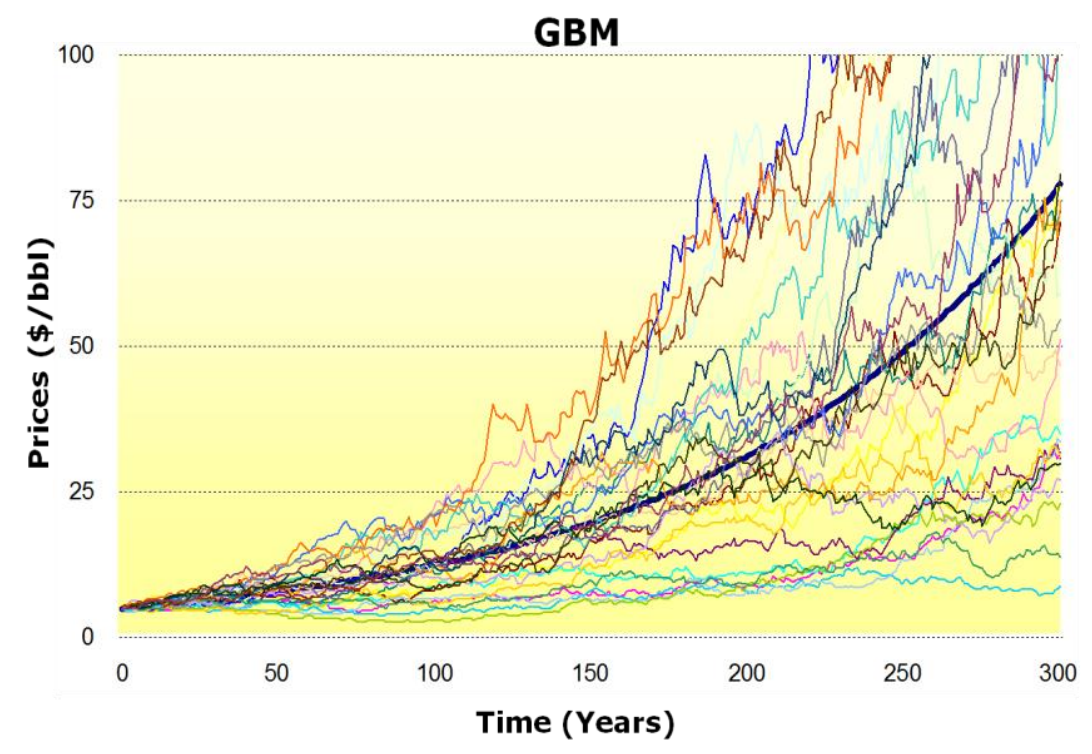

Figure 4.8: Geometric Brownian Motion Samples (Dyer, 2011)

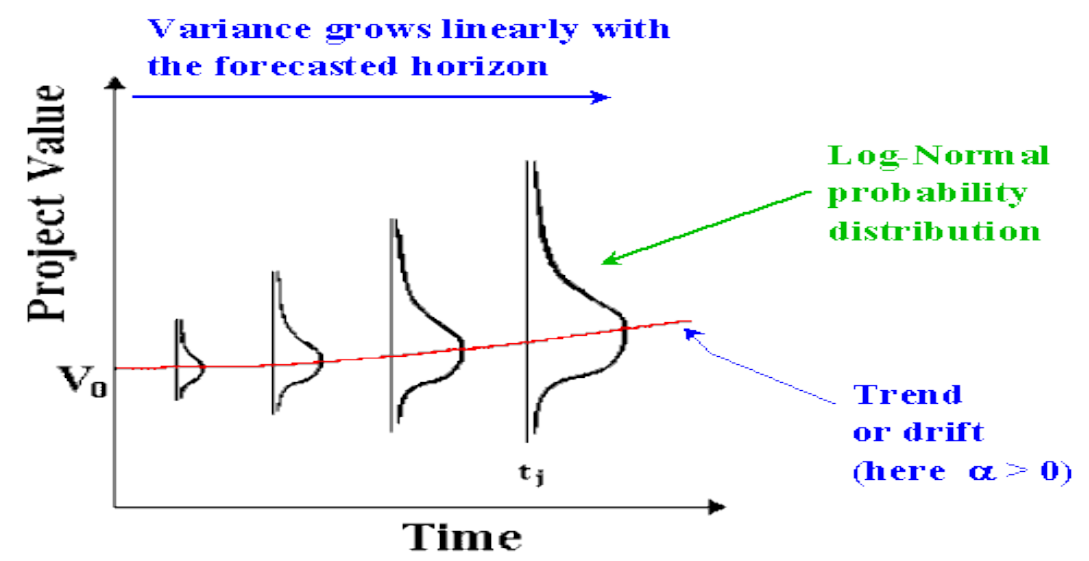

Figure 4.9: Log-normal Diffusion Process of the GBM, Source: www.pucrio.br/marco.ind/stochast.html

Although the GBM is not the most accurate model of stochastic processes, it is the most commonly used due to its practicality and simplicity. In Equation 4.20, which is the GBM general equation, the first term of the right side term of the equation represents the trend or growth rate term, and the second term represents the uncertainty involved or variation term (Dias, 2010b). Substituting $\mathrm{p}=\mathrm{Ln}(\mathrm{P})$ in Equation 4.20, and using Ito's 
Lemma, the result is Equation 4.21, which works with the logarithm of price instead of price (Dixit, 1993). Working with logarithm of prices has 3 advantages. First, it is easier to work with the normal distribution of logarithm of price rather than the log-normal distribution of price. Second, it is simpler to model the GBM using Monte Carlo simulation using logarithm of prices. Third, it is easier to estimate drift and volatility since the two parameters are independent of price. Equation 4.22 is the discrete equation to forecast crude prices.

$$
\begin{aligned}
& d p=d(\ln P)=\left(\alpha-\frac{1}{2} \sigma^{2}\right) d t+\sigma d z \\
& P(t)=P(t-1) * e^{\left(\alpha-\frac{1}{2} \sigma^{2}\right) * \Delta t+\sigma * N(0,1) \sqrt{\Delta t}}
\end{aligned}
$$

\subsection{The GBM Formulation}

Table 4.16 lists the Heavy crude GBM modeling parameters, initial crude price, the minimum Heavy crude price, drift, long-run mean equilibrium and volatility; these parameters were estimated using weekly historical data (1978-2010), and then converted to annual data, so they are applicable for the study. Table 4.17 shows a sample of standard normal distribution $(\mathrm{N}(0,1))$. Substituting the parameters from Tables 4.16 and 4.17 into Equation 4.22 and using time increment of one year, the Heavy crude prices are forecasted as shown in Table 4.18 and Figure 4.10. Table 4.19 shows the correlated prices for the four remaining crudes.

Table 4.16: The Heavy Crude GBM Modeling Parameters

\begin{tabular}{lcl} 
& \multicolumn{2}{c}{ Heavy Crude } \\
$P(0)$ & 56.23 & Initial Price \\
Pmin & 40.00 & Minimum Price \\
$\alpha$ & 0.23 & Drift \\
$P^{*}$ & 56.23 & Long run average Price \\
$\sigma$ & 0.42 & Volatility
\end{tabular}


Table 4.17: Standard Normal Distribution

\begin{tabular}{|lcccccc|}
\hline Time & 0 & 1 & 2 & 3 & 4 & 5 \\
$\varepsilon_{\mathrm{t}}$ & 0.0000 & -0.1992 & 0.2107 & 0.5661 & 1.3907 & -1.4403 \\
Mean & 0 & 0 & 0 & 0 & 0 & 0 \\
S.D. & 1.00 & 1.00 & 1.00 & 1.00 & 1.00 & 1.00 \\
\hline
\end{tabular}

Table 4.18: The GBM Forecasting Model

\begin{tabular}{|lcccccc|}
\hline Year & 0 & 1 & 2 & 3 & 4 & 5 \\
Oil Prices & 56.23 & 59.45 & 74.73 & 109.16 & 225.95 & 141.37 \\
\hline
\end{tabular}

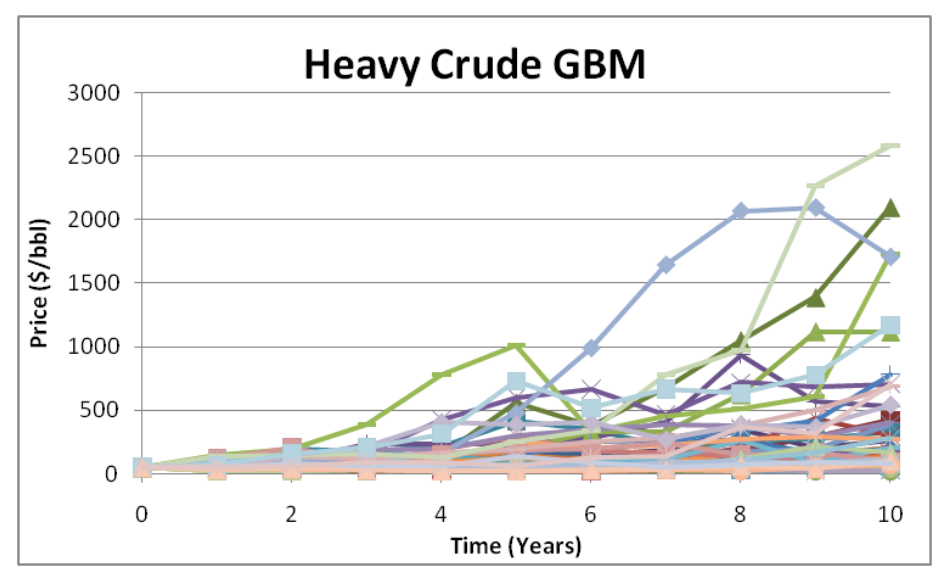

Figure 4.10: The GBM Forecasting Model

Table 4.19: The GBM Correlated Price Forecasts for the Medium, Light, X-Light, and XX-Light

\begin{tabular}{|lcccccc|}
\hline Year & 0 & 1 & 2 & 3 & 4 & 5 \\
Medium Crude Price & 57.86 & 105.27 & 123.09 & 217.70 & 264.34 & 460.54 \\
Light Crude Price & 59.14 & 42.01 & 43.77 & 45.24 & 80.75 & 123.22 \\
X-Light Crude Price & 62.50 & 62.11 & 94.17 & 145.62 & 376.16 & 556.06 \\
XX-Light Crude Price & 60.21 & 77.83 & 130.59 & 116.12 & 159.20 & 109.84 \\
\hline
\end{tabular}




\subsubsection{Mean Reversion Model (MRM)}

\subsection{Mean Reversion Model, Background}

It is believed that crude prices are correlated with crude marginal cost of production. Consequently, the Mean Reversion Model is a more logical forecasting approach than Geometric Brownian Motion. The MRM model allows the response of supply to price changes. For example, producers increase production in response to higher prices to boost their profit, and decrease production in the case of low prices to avoid losses; these decisions force crude oil prices to revert to a long- run equilibrium price. Figure 4.11 shows a sample path of the MRM; how price reverts to a long-run equilibrium value with time. There are different types of Mean Reversion model; the basic and most common model is known as the Ornstein-Uhlenbeck model. Equation 4.23 shows the Ornstein-Uhlenbeck formula. In this formula, the drift is controlled by (P*-P) term. As a result, the drift is positive if the current price is lower than the long-run equilibrium price $\left(\mathrm{P}^{*}\right)$ and negative if the current price is higher than the long-run equilibrium price $\left(\mathrm{P}^{*}\right)$. In other words, the volatile prices are pulled toward the long-run mean price (Dias, 2010c). Moreover, the greater the difference between the current price and long-run equilibrium price, the greater the tendency of the price to revert to the longrun equilibrium price. The MRM is different from the GBM in the drift term, as described earlier, and volatility. Unlike the GBM, the volatility in the MRM does not grow proportionally with time; instead, it grows initially then stabilizes at a specific value. Volatility is bounded in the case of the MRM (Dias, 2010c).

Working with the logarithm of prices is preferable, similar to the GBM, for Monte Carlo simulation and parameter estimation. As mentioned in the GBM section, the logarithm of price has a normal distribution, which is easier to deal with than log-normal distribution (Dias, 2010c). Moreover, parameter estimation is independent of crude price 
when working with the logarithm of prices. Substituting $x=\ln (P)$ in Equation 4.23, results in Equation 4.24, which is the MRM formula for the logarithm of prices (Dias, 2010c).

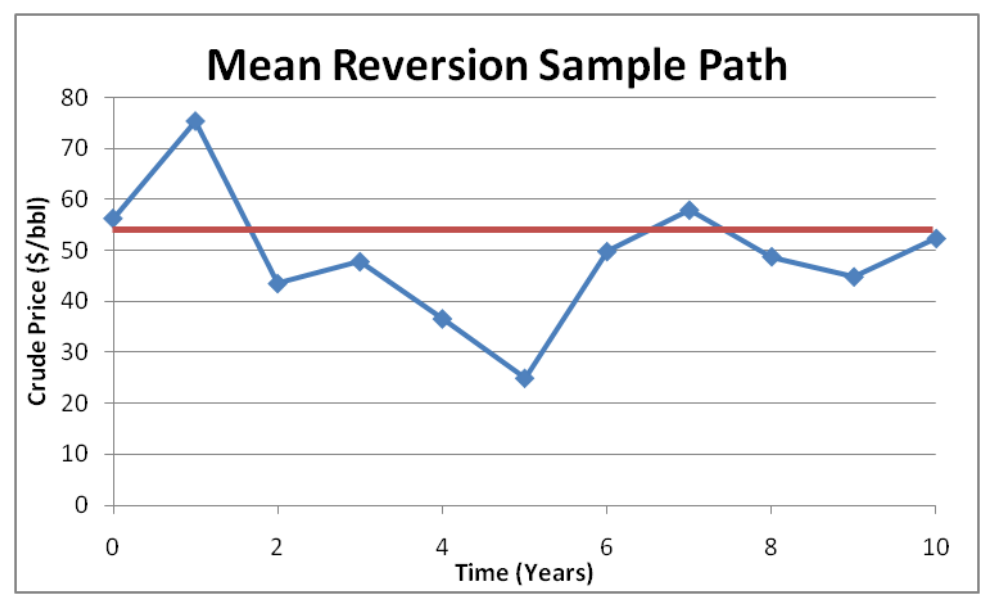

Figure 4.11: Mean Reversion Sample Path

$d P=\eta * P\left(P^{*}-P\right) d t+\sigma * P * d z$

Where

$\mathrm{P} *$ is the long-run equilibrium prices

$\eta$ is reversion speed

$\mathrm{dz}($ Wiener increment $)=\varepsilon \mathrm{dt}^{1 / 2}$

$\varepsilon=$ Standard Normal $=\mathrm{N}(0,1)$

$\sigma=$ Volatility of $\mathrm{P}$

$d x=\eta\left(x^{*}-x\right) d t+\sigma d z$

Where

$\mathrm{x}=\ln (\mathrm{P})$

\subsection{Mean Reversion Model Formulation}

The Mean Reversion Model implemented is the Ornstein-Uhlenbeck type and works with the logarithm of price (x (t)). Equation 4.25 is the main forecasting formula, 
which is function of the logarithm of price at a given time $(\mathrm{x}(\mathrm{t}))$ and variance of the logarithm of price at the same time period $(\operatorname{Var}[\mathrm{x}(\mathrm{t})])$. The logarithm of price $\mathrm{x}(\mathrm{t})$ and its variance $\operatorname{Var}[\mathrm{x}(\mathrm{t})]$ are calculated for every time period using equations 4.27 and 4.28 , respectively. Substituting the parameters from Table 4.20, standard normal distribution $(\mathrm{N}(0,1))$ from Table 4.21, and the calculated $\mathrm{x}(\mathrm{t})$ and $\operatorname{Var}[\mathrm{x}(\mathrm{t})]$ in Equation 4.25, crude prices are forecasted as shown in Table 4.22. Although the study covers for ten years, the calculations shown here are for the first five years for illustration. Similar to the GBM, a minimum price for the Heavy crude is set at $\$ 40.00$; the MAX function is to implement the floor price of $\$ 40.00$. Table 4.23 shows price forecasts for the remaining four crudes using the correlations calculated in Crude Prices Correlation chapter.

$$
\begin{aligned}
P_{t} & =e^{\left\{x_{t}-\frac{1}{2} \operatorname{Var}\left[x_{t}\right]\right\}} \\
x^{*} & =\ln \left(P^{*}\right) \\
x_{t} & =x_{t-1} * e^{[-\eta * \Delta t]}+x^{*} *\left(1-e^{[-\eta * \Delta t]}\right)+\sigma * \sqrt{\left(1-e^{[-2 \eta * \Delta t]}\right) /(2 \eta)} * \\
N(0,1) & \operatorname{Var}\left[x_{t}\right]=\left(1-e^{[-2 \eta * t]}\right) * \frac{\sigma^{2}}{2 \eta}
\end{aligned}
$$

Table 4.20: The Heavy Crude MRM Forecasting Parameters

\begin{tabular}{lcl} 
& \multicolumn{2}{c}{ Heavy Crude } \\
Po & 56.23 & Initial Price \\
Pmin & 40.00 & Minimum Price \\
$\eta$ & 1.50 & Reversion Speed \\
$\mathbf{P}^{*}$ & 56.23 & Long-run Average Price \\
Ln(P*) & 4.03 & \\
$\boldsymbol{\sigma}$ & 0.59 & Volatility
\end{tabular}

Table 4.21: Standard Normal Distribution 


\begin{tabular}{|lcccccc|}
\hline Time & 0 & 1 & 2 & 3 & 4 & 5 \\
$\varepsilon_{\mathrm{t}}$ & 0.0000 & -0.1992 & 0.2107 & 0.5661 & 1.3907 & -1.4403 \\
Mean & 0 & 0 & 0 & 0 & 0 & 0 \\
S.D. & 1.00 & 1.00 & 1.00 & 1.00 & 1.00 & 1.00 \\
\hline
\end{tabular}

Table 4.22: The Heavy Crude Mean Reversion Model

\begin{tabular}{|lcccccc|}
\hline Year & 0 & 1 & 2 & 3 & 4 & 5 \\
$\mathbf{X}(\mathbf{t})=$ "In(P)" & 4.03 & 3.28 & 3.53 & 4.11 & 3.92 & 3.59 \\
$\operatorname{Var}[\mathbf{X}(\mathbf{t})]$ & 0.00 & 0.11 & 0.12 & 0.12 & 0.12 & 0.12 \\
Oil Prices "P" & 56.23 & 40.00 & 40.00 & 57.52 & 47.35 & 40.00 \\
\hline
\end{tabular}

Table 4.23: Correlated Mean Reversion Models for the Medium, Light, X-Light, and XX-Light Crudes

\begin{tabular}{|lllllll|}
\hline Year & 0 & 1 & 2 & 3 & 4 & 5 \\
Medium Crude Price & 57.58 & 68.32 & 88.61 & 41.66 & 51.76 & 72.13 \\
Light Crude Price & 56.40 & 51.07 & 94.93 & 87.00 & 70.83 & 116.66 \\
X-Light Crude Price & 59.15 & 63.68 & 71.11 & 61.25 & 58.18 & 44.47 \\
XX-Light Crude Price & 65.65 & 47.37 & 59.57 & 78.81 & 78.36 & 77.70 \\
\hline
\end{tabular}

\subsubsection{Mean Reversion with Jumps Model (MRJM)}

\subsection{Mean Reversion with Jumps Model, Background}

Mean Reversion with Jumps relates price forecast to information arrival. Based on the model, there are two types of information, normal and abnormal information. Normal information (daily news) causes the smooth changes in crude oil prices and the abnormal information (political events, wars, etc.) causes jumps in prices. With the current political and natural events, the Mean Reversion with Jumps Model is the most logical approach to forecasting crude prices. The MRJM models the smooth changing in crude prices with the Mean Reversion Model, and the price jumps with the Poisson process (Jumps). Figure 4.12 shows Brent crude monthly prices between 1970 and 2000; the reversion trend and jumps in prices are clear. The Poisson process is a statistical process where the time intervals between successive jumps are exponentially distributed, which means that the 
time intervals of successive jumps are independently and identically distributed (Dias, 2010a).

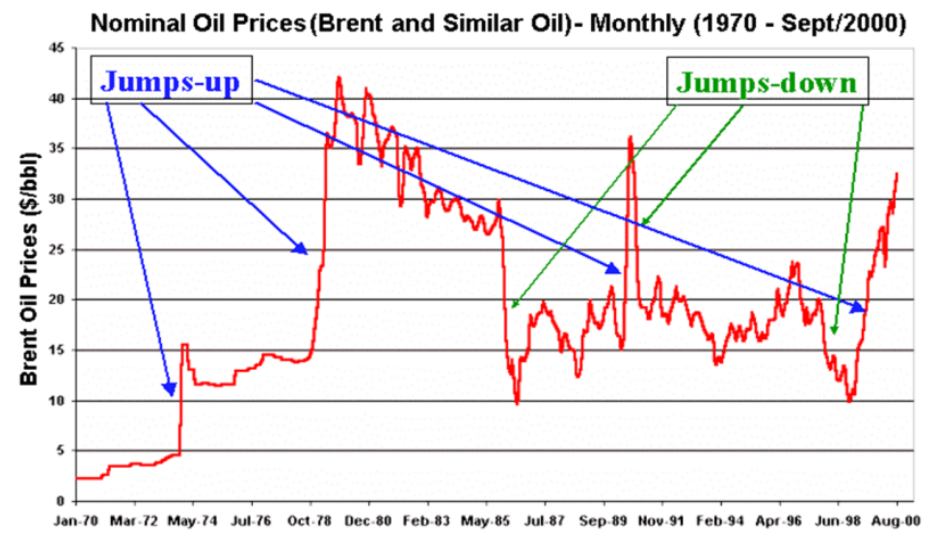

Figure 4.12: Brent Crude Monthly Prices (1970-2000), Source: $\underline{w w w . p u c-}$ rio.br/marco.ind/rev-jump.html

Equation 4.29 is the general form of the Mean Reversion with Jumps Model for crude prices. A new term in the equation (dq), which is not used in the GBM or the MRM formulas, represents Poisson distribution. This distribution is responsible for the jumps in the model; dq is zero all the time (no Jumps), but with frequency of $\lambda$ a 1 is assigned to the term causing a jump in price forecasts. The probability of dq to acquire value of 1 is $\lambda^{*} \Delta \mathrm{t}$. The presence of $\Delta \mathrm{t}$ in the probability term stresses on the significance of assigning a proper small $\Delta \mathrm{t}$ to result in a reasonable small probability. Small probability is favored to prevent the occurrence of more than one jump in the same time period. A distinct characteristic of MRJM is that the Poisson term (dq) and the Wiener increment term (dz) are independent of each other; in other words, the price jumps are independent of the smooth mean reversion changes in crude prices. This characteristic is perfect for the MRJM, since abnormal news arrival is independent of the normal news arrival. Another important MRJM parameter is the jump size $(\phi)$; the jump size can be a discrete number 
or random variable. The jump size determines the direction of the jumps by its sign; if the jump size is positive, the price changes are up and vice versa (Dias, 2010a).

$$
d P=\eta * P *\left(P^{*}-P\right) d t+\sigma * P * d z+P * d q
$$

Where

$\mathrm{dq}$ is the Poisson term, which represents the jumps

$\mathrm{P}^{*}$ is the long-run equilibrium prices

$\eta$ is reversion speed

$\mathrm{dz}($ Wiener increment $)=\varepsilon \mathrm{dt}^{1 / 2}$

$\varepsilon=$ Standard Normal $=\mathrm{N}(0,1)$

$\sigma=$ Volatility of $\mathrm{P}$

The Mean Reversion with Jumps model obeys economical and statistical logics through the mean reversion part of the model, and through the jumps, respectively. The economical logic appears in the reversion process, which allows the supply response to price fluctuations. The statistical logic appears in the flat long tail of the price probability density function; the long flat tail is resulted from the jumps. On the other hand, it is difficult to estimate the MRJM parameters. Similar to the GBM and the MRM, it is preferable to work with the logarithm of crude prices, since it facilitates the simulation process (Dias, 2010a).

Equation 4.30 is the MRJM formula for the logarithm of crude prices; the equation states that there are reversion forces pulling the logarithm of prices (x) toward the long-run mean level $(\ln (\mathrm{x}))$, and there is a jumping force with a magnitude of $\varphi$ and a frequency of $\lambda$. Unlike the GBM and the MRM, variable $x(t)$ is not normally distributed due to the fact that jumps add a flatter tail to the normal distribution. (Dias, 2010d)

$$
d x=d[\ln (P)]=\eta *\left[x^{*}-x\right] * d t+\sigma * d z+\Phi * d q
$$

Where 
$\mathrm{X}^{*}=\ln \left(\mathrm{P}^{*}\right)$

$\Phi$ is the jump size

Choosing an appropriate $\Delta \mathrm{t}$ is critical for the MRJM. Although the mean reversion part of the model is exact which allows the use of large $\Delta \mathrm{t}$, using large $\Delta \mathrm{t}$ is problematic for the jump part of the model. Using large $\Delta t$ increases the probability of two jumps to arrive at the same period. Equation 4.31 presents the probability of $n$ jumps to arrive at the same period with a jump frequency of $\lambda$. Substituting $(n=2, \lambda=1$, and $t=$ 1 year) in Equation 4.31, the probability of two jumps occurring at the same period is $18.4 \%$, which is not negligible. On the other hand, the probability of two jumps occurring in the same period reduces sharply to $0.02 \%$, which is negligible, by using ( $\mathrm{n}=2, \lambda$ $=0.25$, and $\mathrm{t}=0.08$ year $(1 \mathrm{month}))$. The smaller the time period, the more accurate the forecasting is (Dias, 2010d).

$$
\text { Probability }=\frac{e^{-\lambda t}}{n !} *(\lambda t)^{n}
$$

\subsection{Mean Reversion with Jumps Model Formulation}

The Heavy crude prices are modeled using the MRJM, and then the remaining crude prices are correlated by the correlations calculated in the crude price correlation section. To ensure the high accuracy of the model, weekly time steps ( 0.02 year) are used to forecast ten years of crude prices. Table 4.24 lists the general parameters required for the MRJM. Volatility and reversion speed are estimated using 2004-2010 weekly historical data. However, to maintain the MRJM model "mean equivalent" to the GBM and the MRM, the long-run mean price is estimated from 2004-2010 annual historical data then converted to weekly data. Table 4.25 shows jump parameters. As advised by Dr. Marco Dias, it is better to use reasonable parameters for jumps rather than estimate them from poor data. Dr. Dias suggested $0.125,0.693$, and 0.523 for $\lambda$, $\phi$ and $E\left[\phi^{2}\right]$ 
respectively, as shown in Table 4.25 (Dias, 2010d). The Poisson process is modeled in Table 4.26; Equations 4.35 and 4.36 are used to calculate JumpSizeUp(t) and JumpSizeDown(t) respectively. The two equations are the multiplication of Poisson distributions for the specific time period with frequency of $\lambda^{*} \Delta t$ and jump size whether up or down. The jump size, the last row in Table 4.26, is the sum of the "JumpSizeUp(t)" and "JumpSizeDown(t)". Table 4.27 is a sample of the price forecast model. In the forecast process, the logarithm of prices $(\mathrm{x}(\mathrm{t}))$ and variance of the logarithm of prices $(\operatorname{Var}[\mathrm{x}(\mathrm{t})])$ are calculated from Equations 4.34 and 4.37, respectively. Substituting the values of $\mathrm{x}(\mathrm{t})$ and $\operatorname{Var}[\mathrm{x}(\mathrm{t})]$ into Equation 4.32, crude prices are forecasted as shown in Table 4.27. To use the weekly forecasted crude prices in the study, which is built to handle annual crude prices, weekly prices under one year are averaged as shown in Table 4.28 to represent the average price for that year. Moreover, the prices of the remaining crudes are correlated to the forecasted Heavy crude prices (shown in Table 4.28) using the correlation process explained in Crude Prices Correlations chapter (Dias, 2010d).

$$
\begin{gathered}
P_{t}=e^{\left\{x_{t}-\frac{1}{2} \operatorname{Var}\left[x_{t}\right]\right\}} \\
x^{*}=\ln \left(P^{*}\right) \\
x_{t}=x_{t-1} * e^{[-\eta * \Delta t]}+x^{*} *\left(1-e^{[-\eta * \Delta t]}\right)+\sigma * \sqrt{\left(1-e^{[-2 \eta * \Delta t]}\right) /(2 \eta)} * \\
N(0,1)+\operatorname{JumpSizeUp}_{(t)}+\operatorname{JumpSizeDown}(t) \\
\operatorname{JumpSizeUp}_{(t)}=\operatorname{Poisson}(\lambda * \Delta t) * \Phi_{U p} \\
\operatorname{JumpSizeDown}_{(t)}=\operatorname{PoisSon}(\lambda * \Delta t) * \Phi_{\mathrm{Down}} \\
\operatorname{Var}\left[x_{t}\right]=\left(1-e^{[-2 \eta * t]}\right) * \frac{\left(\sigma^{2}+\left(\lambda_{u p}+\lambda_{\text {Down }}\right) * E\left[\Phi^{2}\right]\right)}{2 \eta}
\end{gathered}
$$

Table 4.24: Mean Reversion with Jumps Modeling Weekly Parameters 


\begin{tabular}{|lcl|}
\hline \multicolumn{1}{|c}{ Parameter } & Value & \multicolumn{1}{c|}{ Unit } \\
$\mathbf{P}(\mathrm{t}=\mathbf{0})$ & 56.23 & $\$ / \mathrm{bbl}$ \\
$\Delta \mathrm{t}$ & 0.02 & years \\
Time Horizon & 10.00 & years \\
Volatility $\sigma$ & 0.05 & \\
Long-Run Mean Price $\left(\mathbf{P}^{\star}\right)$ & 56.23 & $\$ / \mathrm{bbl}$ \\
Ln(P) & 4.03 & $\$ / \mathrm{bbl}$ \\
Reversion Speed $\eta$ & 0.01 & \\
\hline
\end{tabular}

Table 4.25: Jumps Parameters

\begin{tabular}{|ccl|}
\hline & \multicolumn{2}{c|}{ Jump Parameters } \\
$\lambda$ & 0.125 & Jump Frequency \\
$\lambda^{*} \Delta \mathrm{t}$ & 0.002 & Jump Probability \\
$\phi$ & 0.693 & Jump Size Up \\
$\phi$ & -0.693 & Jump Size Down \\
$\mathrm{E}\left[\phi^{2}\right]=$ & 0.523 & Expected Value of Jump Size \\
\hline
\end{tabular}

Table 4.26: Poisson Process

\begin{tabular}{|c|c|c|c|c|c|c|c|c|c|c|}
\hline Time (Years) & 0 & 0.02 & 0.04 & 0.06 & 0.08 & 0.10 & 0.12 & 0.13 & 0.15 & 0.17 \\
\hline JumpSizeUp(t) & & 0.00 & 0.00 & 0.00 & 0.00 & 0.69 & 0.00 & 0.00 & 0.00 & 0.00 \\
\hline JumpSizeDown(t) & & 0.00 & 0.00 & 0.00 & 0.00 & 0.00 & 0.00 & 0.00 & 0.00 & 0.00 \\
\hline Jump Size (Poisson) & & 0.00 & 0.00 & 0.00 & 0.00 & 0.69 & 0.00 & 0.00 & 0.00 & 0.00 \\
\hline
\end{tabular}

Table 4.27: Mean Reversion with Jumps Model Forecasts

\begin{tabular}{|lcccccccccc|}
\hline Time (Years) & $\mathbf{0}$ & 0.02 & 0.04 & 0.06 & 0.08 & 0.10 & 0.12 & 0.13 & 0.15 & 0.17 \\
\cline { 2 - 9 } $\mathbf{P}(\mathbf{t})$ & $\mathbf{5 6 . 2 3}$ & 56.54 & 56.24 & 56.28 & 56.37 & 56.33 & 56.42 & 28.13 & 27.94 & 28.12 \\
$\mathrm{~N}(\mathbf{0}, \mathbf{1})$ & & 0.99 & -0.61 & 0.26 & 0.41 & 0.08 & 0.40 & -0.22 & -0.83 & 1.10 \\
$\mathbf{X}(\mathbf{t})$ Poisson & $\mathbf{4 . 0 3}$ & 4.04 & 4.03 & 4.03 & 4.04 & 4.04 & 4.04 & 3.35 & 3.34 & 3.35 \\
\hline
\end{tabular}

Table 4.28: Annual Forecasted Crude Prices Using Mean Reversion with Jumps

\begin{tabular}{|lccccccccccc|}
\hline & \multicolumn{8}{c|}{ Annual Average Correlation } \\
Time (Years) & 0 & 1 & 2 & 3 & 4 & 5 & 6 & 7 & 8 & 9 \\
$\mathbf{P}(\mathrm{t})_{\text {Heavy }}$ & 56.23 & 40.48 & 49.21 & 48.63 & 45.46 & 44.14 & 30.86 & 22.14 & 21.68 & 19.67 & 9.86 \\
$\mathbf{P}(\mathrm{t})_{\text {Medium }}$ & 58.20 & 41.63 & 51.45 & 49.70 & 47.01 & 45.65 & 32.09 & 23.82 & 23.68 & 21.04 & 10.90 \\
$\mathbf{P}(\mathrm{t})_{\text {Light }}$ & 60.18 & 43.31 & 51.97 & 51.09 & 47.80 & 48.34 & 34.11 & 24.57 & 24.95 & 21.89 & 12.79 \\
$\mathbf{P}(\mathrm{t})_{\text {X-Light }}$ & 59.71 & 45.97 & 52.92 & 51.89 & 53.11 & 51.78 & 35.26 & 27.87 & 25.24 & 20.09 & 10.16 \\
$\mathbf{P}(\mathrm{t})_{\text {XX-Light }}$ & 60.59 & 50.14 & 52.47 & 52.62 & 51.00 & 48.46 & 33.39 & 23.47 & 32.22 & 23.13 & 7.54 \\
\hline
\end{tabular}

\subsection{Correlating the Price Models}

Typically, decision analysis studies within the oil industry deal with prices of one crude type. Unlike most of the decision analysis studies within the oil industry, our study 
involves five different crude grades and prices. Although all parameters required to build the various price forecasting models are estimated for all the five crude grades, it is not feasible to model the prices of each crude grade independently. Modeling different crude grade prices independently results in unrealistic forecasts since one set of prices can jump upward and at the same time another set can jump downward, which is impossible. Moreover, historical crude prices show high correlation factors, as shown in Table 4.29, between the Heavy-Medium, and the Heavy-Light crudes. Figure 4.13 is a regression graph of the Medium and the Heavy crude prices; the value of square of error term $\left(\mathrm{R}^{2}\right)$ at 0.9993 shows the strong correlation between the two sets of prices. These historical facts emphasize the significance of correlating the crude prices instead of modeling each one independently. Crude prices are correlated based on 1989-2010 historical crude prices. Regression analysis is used to correlate the crude prices; regression parameters and standard deviation are estimated for the data to be correlated. The standard deviation of the correlated data is needed to estimate the residual, which is the error or the difference between the actual data and simulated data. Equation 4.38 shows the typical regression formula; $\mathrm{Y}$ is the dependent variable, $\mathrm{X}$ is the independent variable, $a$ is the slope, $b$ is the y-axis intercept, and $\varepsilon$ is the residual or the error term. In this study, the Heavy crude price is the independent variable. In other words, all different crude prices are calculated based on the Heavy crude price. In order to correlate the different crude prices to those of the Heavy crude, a, b, and $\varepsilon$ must be estimated.

$$
Y=a * X+b+\varepsilon
$$

Table 4.29: Crude Price Correlation Factors

\begin{tabular}{|lc|}
\hline & Correlation Factor \\
Heavy-Medium & 0.9996 \\
Heavy-Light & 0.9984 \\
\hline
\end{tabular}




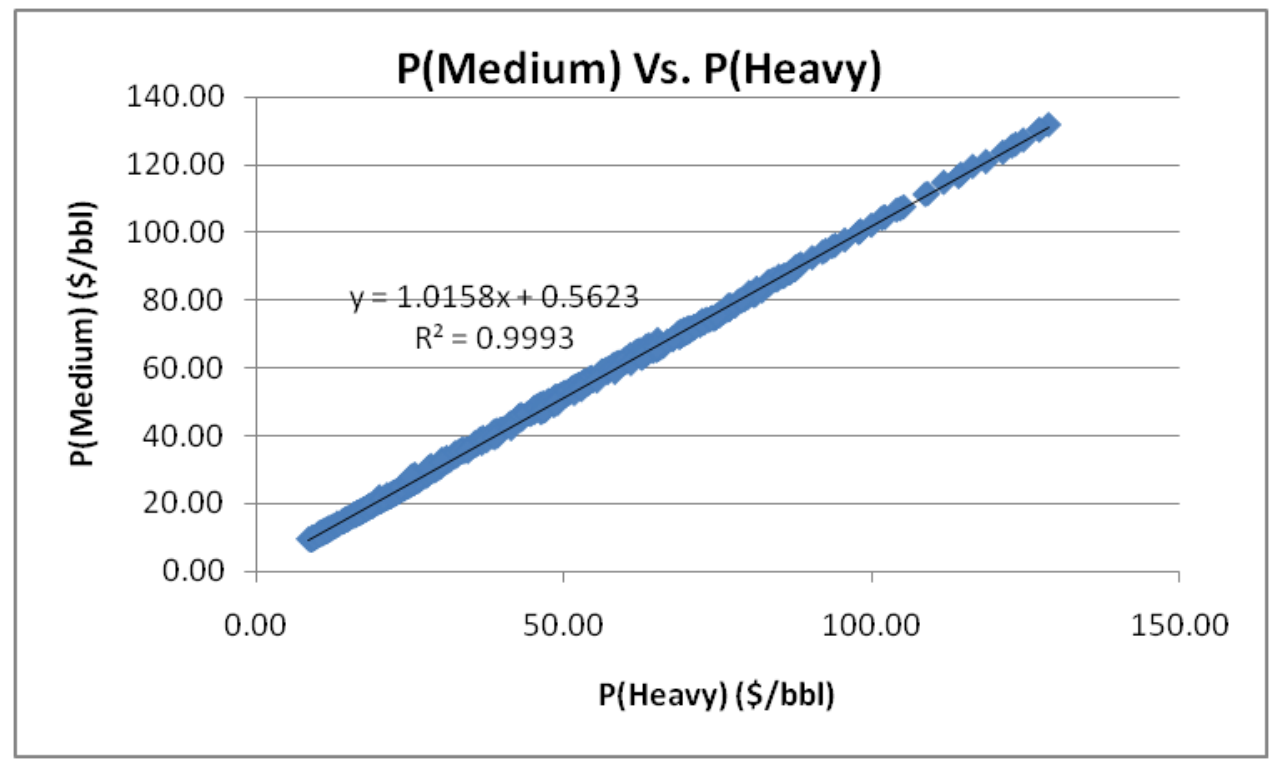

Figure 4.13: The Heavy and the Medium Crude Prices Correlation Using 1989-2010 Weekly Historical Data

Using historical data from Table 4.30, the Medium and the Light crude prices are plotted as a function of the Heavy crude prices as shown in Figure 4.14 and Figure 4.15. From these two figures, linear lines are fitted through the data with equations shown in the figures. Then, the regression parameters, slope of the line and y-intercept, are calculated and recorded in Table 4.31. Using the STEYX function in EXCEL, standard errors (standard deviations) are calculated for every two correlated crude prices as shown in Table 4.32. Table 4.33 shows the residual between the listed and the Heavy crude grade; the residual is a normal distribution with a mean of zero and standard deviation of the standard error calculated in Table 4.32.

The regression parameters (slope, intercept and standard error) for the two missing crudes are calculated by adding the differential value of a parameter between the known crudes to the preceding crude. For example, the slope value (a) for the X-light crude is calculated by adding the difference of slope values between the Medium and the 
Heavy crudes $(1.03-1.01=\mathbf{0 . 0 2})$ to the slope value of the Light crude, so $1.03+0.02=$ 1.05 .

Table 4.30: Annual Historical Prices for the Light, Medium and Heavy Crudes

\begin{tabular}{|c|c|c|c|}
\hline Date & $\begin{array}{l}\text { Light Crude Price } \\
\text { (\$/bbl) }\end{array}$ & $\begin{array}{l}\text { Medium Crude Price } \\
\qquad(\$ / b b l)\end{array}$ & $\begin{array}{c}\text { Heavy Crude Price } \\
(\$ / \mathrm{bbl})\end{array}$ \\
\hline Jan 06, 1989 & 13.15 & 12.30 & 11.90 \\
\hline Jan 05, 1990 & 18.40 & 17.55 & 17.15 \\
\hline Jan 04, 1991 & 24.00 & 22.00 & 20.00 \\
\hline Jan 03, 1992 & 15.90 & 14.25 & 13.15 \\
\hline Jan 01, 1993 & 16.80 & 15.40 & 14.40 \\
\hline Jan 07, 1994 & 12.40 & 11.20 & 10.10 \\
\hline Jan 06, 1995 & 16.63 & 15.73 & 15.13 \\
\hline Jan 05, 1996 & 18.20 & 17.40 & 17.05 \\
\hline Jan 03, 1997 & 22.98 & 21.93 & 21.08 \\
\hline Jan 02, 1998 & 15.50 & 14.90 & 14.00 \\
\hline Jan 01, 1999 & 10.03 & 9.63 & 9.28 \\
\hline Jan 07, 2000 & 23.45 & 22.85 & 22.10 \\
\hline Jan 05, 2001 & 20.90 & 20.30 & 19.40 \\
\hline Jan 04, 2002 & 18.90 & 18.55 & 18.15 \\
\hline Jan 03, 2003 & 27.39 & 26.44 & 25.69 \\
\hline Jan 02, 2004 & 27.08 & 26.13 & 25.38 \\
\hline Jan 07, 2005 & 34.05 & 30.60 & 27.95 \\
\hline Jan 06, 2006 & 55.01 & 52.86 & 50.41 \\
\hline Jan 05, 2007 & 53.48 & 51.53 & 49.83 \\
\hline Jan 04, 2008 & 93.02 & 90.27 & 88.07 \\
\hline Jan 02, 2009 & 35.21 & 32.16 & 30.16 \\
\hline Jan 01, 2010 & 75.21 & 74.01 & 73.56 \\
\hline Dec 24, 2010 & 90.03 & 88.33 & 86.88 \\
\hline
\end{tabular}




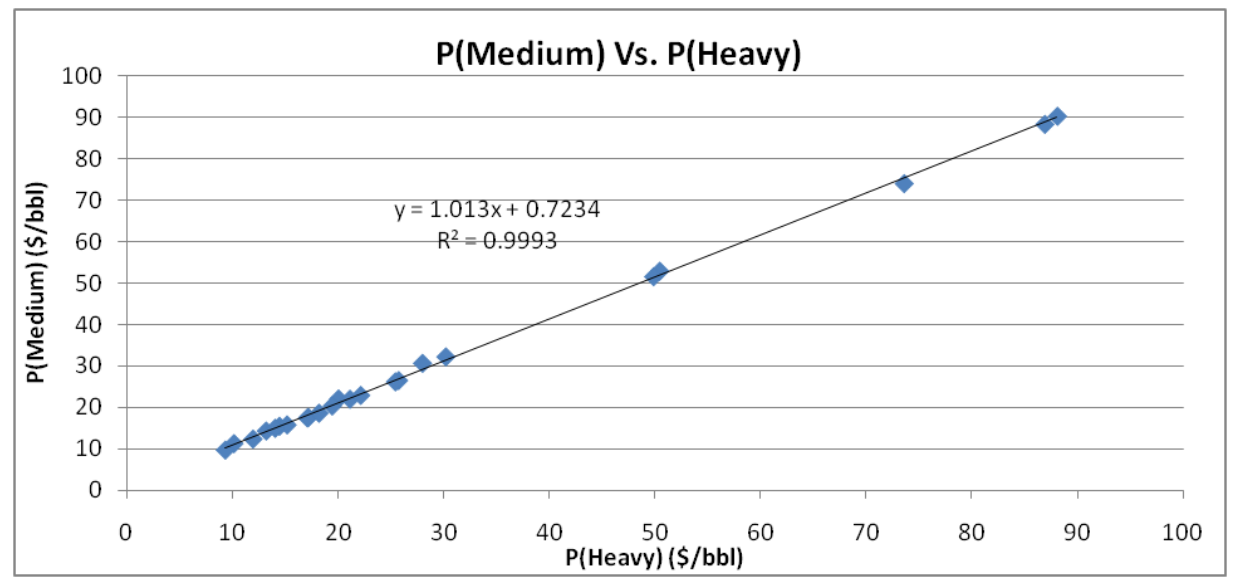

Figure 4.14: The Heavy and Medium Price Correlation Using 1989-2010 Annual Historical Data

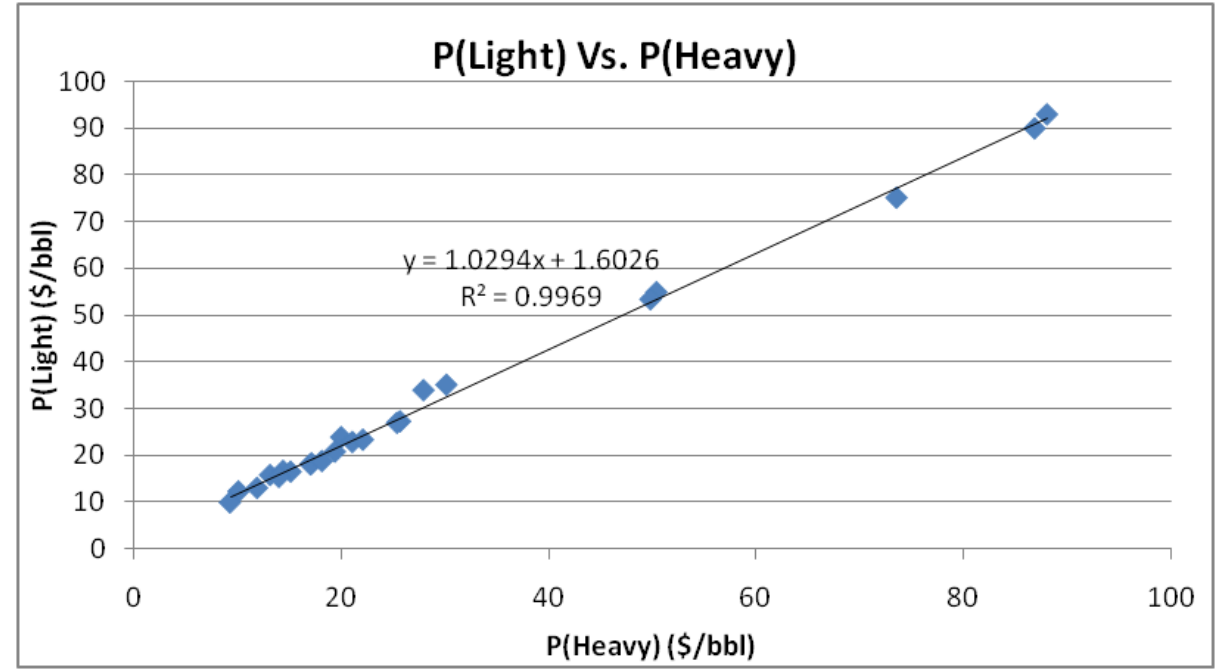

Figure 4.15: The Heavy and Light Crude Price Correlation Using 1989-2010 Annual historical Data

Table 4.31: Crude Price Correlation Parameters

\begin{tabular}{|lcc|}
\hline & a (Slope) & b (Intersection) \\
Medium-Heavy & 1.01 & 0.72 \\
Light-Heavy & 1.03 & 1.60 \\
X-light - Heavy & 1.05 & 2.48 \\
XX-Light - Heavy & 1.08 & 4.24 \\
\hline
\end{tabular}


Table 4.32: Standard Deviation of the Residual

\begin{tabular}{|lr|}
\hline \multicolumn{2}{|c|}{ Standard Error } \\
Medium-Heavy & 0.663 \\
Light-Heavy & 1.398 \\
X-light - Heavy & 2.133 \\
XX-Light - Heavy & 3.602 \\
\hline
\end{tabular}

Table 4.33: Regression Residual by Crude Grades

\begin{tabular}{|c|c|c|c|c|c|c|c|c|c|c|c|}
\hline Time (Years) & 0 & 1 & 2 & 3 & 4 & 5 & 6 & 7 & 8 & 9 & 10 \\
\hline$\varepsilon_{\mathrm{t}}($ Medium $)$ & 0.39 & 0.02 & -0.06 & 0.49 & -0.41 & -0.05 & -0.55 & 0.44 & -1.39 & -1.04 & 0.38 \\
\hline$\varepsilon_{\mathrm{t}}($ Light $)$ & 0.14 & 0.70 & -1.30 & -2.21 & 0.07 & -0.98 & 1.03 & 1.30 & 2.14 & -0.48 & -1.80 \\
\hline$\varepsilon_{t}(X$-Light $)$ & -0.51 & 1.11 & 0.37 & -0.24 & -0.98 & -1.76 & -0.79 & 1.46 & 2.13 & -1.00 & -4.00 \\
\hline
\end{tabular}




\section{CHAPTER 5: INTEGRATED OPTIMIZATION MODEL}

The integrated optimization model is the heart of the study. The integrated model connects different processes to convert the numbers within all the process into meaningful results. This section describes the programming process and the functionality of the integrated optimization model. The topics covered here are objective of the model, assumptions, model flowchart, problem formulation, and model description.

\subsection{Model Assumptions}

Assumptions have been imposed to the study to facilitate the execution and functionality of the models while ensuring the integrity of the results. Here, these assumptions are classified and declared.

\subsubsection{Probabilistic Crude Oil Price Modeling Assumptions}

- One thousand iterations are sufficient to minimize the error in the simulation process in the probabilistic price forecasting approach.

- $\quad$ Crude oil prices are independently and log-normally distributed each year in the Independent Log-normal Distribution price forecasting process.

\subsubsection{Field PARAmeter Estimation Assumptions}

Field parameters were estimated based on data ranges. These parameter ranges, shown in Table 5.1, were generated by industry experts. Fields parameters were estimated because of the sensitivity involved in revealing actual field data (e.g., operating costs, reserves, etc). Implementation of these assumptions and estimation of the parameters are described in the parameter section. 
Table 5.1: $\quad$ Field Parameter Estimation Assumptions

\begin{tabular}{|c|c|c|c|c|c|c|}
\hline Crude Grade & $\begin{array}{c}\text { Reserves Range } \\
\text { MMBBI }\end{array}$ & $\begin{array}{c}\text { Depletion Rate Range } \\
\qquad \%\end{array}$ & $\begin{array}{c}\text { Min Operating Rate } \\
\text { (\% of Depletion) }\end{array}$ & $\begin{array}{c}\text { CAPEX } \\
\text { M\$s/bbl Required }\end{array}$ & $\begin{array}{l}\text { Fixed OPEX } \\
\text { ( } \% \text { of CAPEX) }\end{array}$ & $\begin{array}{c}\text { Variable OPEX } \\
(\$ / \mathrm{bbl})\end{array}$ \\
\hline XX-Light & $100-5,000$ & $2-8$ & 30 & $5,000-10,000$ & 5 & $0.3-1.0$ \\
\hline X-Light & $200-10,000$ & $2-6$ & 30 & $6,000-12,000$ & 5 & $0.5-1.2$ \\
\hline Light & $500-15,000$ & $2-4$ & 30 & $7,000-14,000$ & 5 & $0.7-1.4$ \\
\hline Medium & $1,000-20,000$ & $1-3$ & 30 & $10,000-20,000$ & 5 & $1.0-1.7$ \\
\hline Heavy & $2,000-25,000$ & $1-2$ & 30 & $15,000-25,000$ & 5 & $1.2-2.0$ \\
\hline
\end{tabular}

\subsubsection{General Modeling Assumptions}

- Variable operating costs (OPEX) are independent of crude prices. Although this assumption conflicts with reality, it reduces the computational burden of the optimization model programming.

- Fields are allowed to produce at their maximum potential (no spare capacity).

- Depletion rate is calculated based on the original reserve not the remaining reserve. In other words, the fields are depleted based on their original reserves.

- $\quad$ The Heavy crude fields have large reserves compared to the Lighter crude because the Light crude grades have been produced over time due to their relatively low production costs and higher selling price.

- $\quad$ Each field produces from only one reservoir.

- $\quad$ All fields must be producing at all times (no option to shut down a field). This assumption reflects the fact that the production facility should be producing to ensure a healthy facility and pipelines. Moreover, this assumption reduces the computational burden of the optimization model programming.

- $\quad$ Fields are existing and producing (no development cases). 
- $\quad$ Field production potentials are maintained throughout the life of the fields by drilling and work-over.

- $\quad$ Drilling and work-over costs are included in the OPEX variable costs.

- $\quad$ Operating company is owned by a government.

○ No tax payments

- No royalty payments

$\circ \quad$ No financing nor interest payments

\subsection{Integrated Model Flowchart}

Figure 5.1 summarizes the processes of the integrated model. The process starts by identifying the crude oil pricing forecast approach (deterministic or probabilistic).

In the deterministic approach there are two price forecasting processes. The Flat Price model assigns a single price value for each crude grade throughout the life of the project. The Floating Price model starts with initial crude prices which grow at a constant annual rate. After the prices are forecasted, parameters are input into the model, and total NPV is calculated; an optimizing algorithm calculates the production rate allocation that maximizes the total net present value (NPV).

In the probabilistic approach, there are four price forecasting stochastic processes: Independent Lognormal Distribution, Mean Reverting, Mean Reverting with Jumps and Geometric Brownian Motion, which are discussed in the price modeling chapter. After crude prices are forecasted and parameters are fed into the model, total NPV is calculated and plotted as a probability distribution due to the fact that crude prices are a probability distribution. Powell and Baker (2010) recommended maximizing the mean of the distribution of the objective function in the case of "optimization under-uncertainty" as 
illustrated in Figure 5.2. The final result of the model is the production rate allocation that maximizes the objective function (total NPV or mean of total NPV).

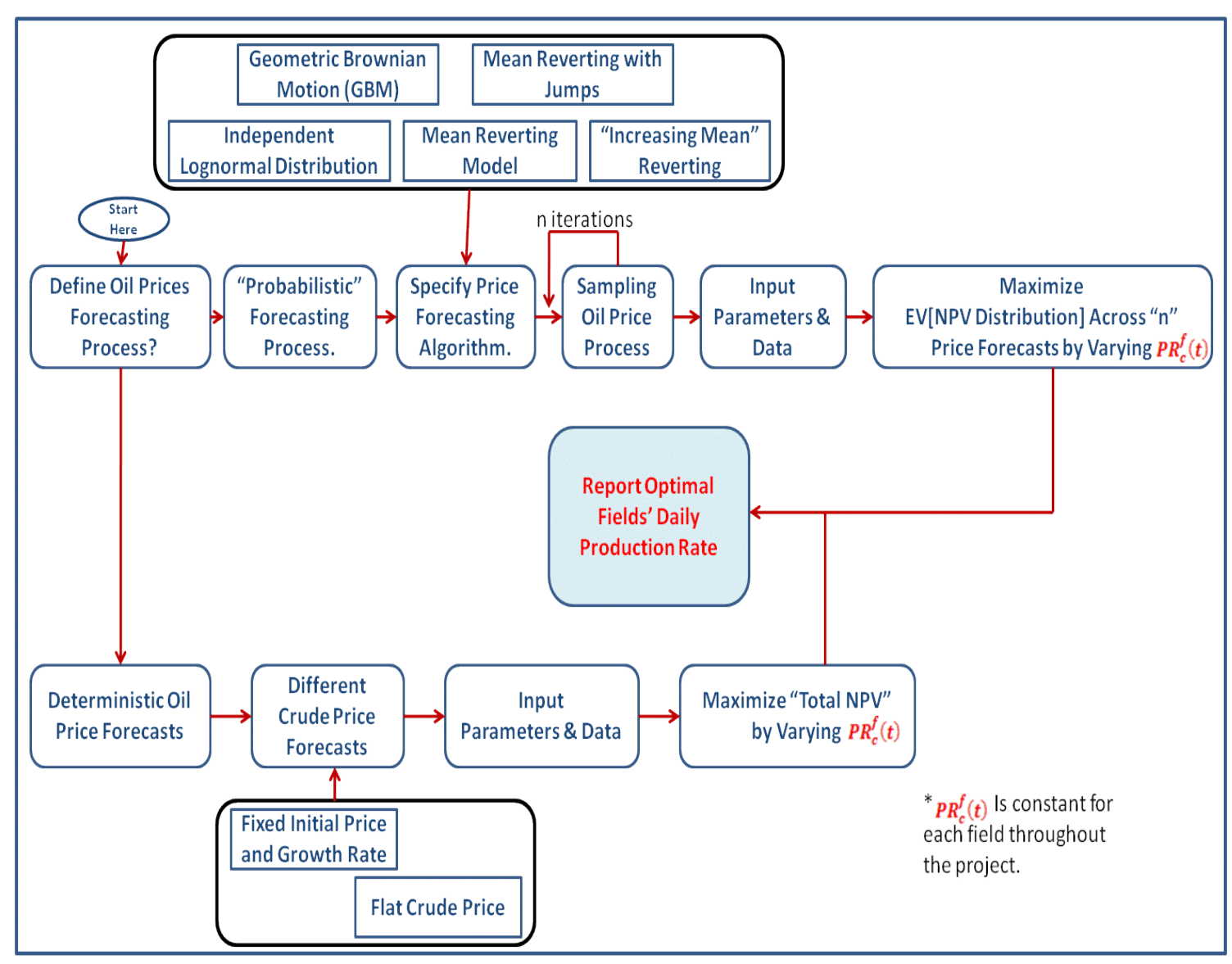

Figure 5.1: Optimization Process 


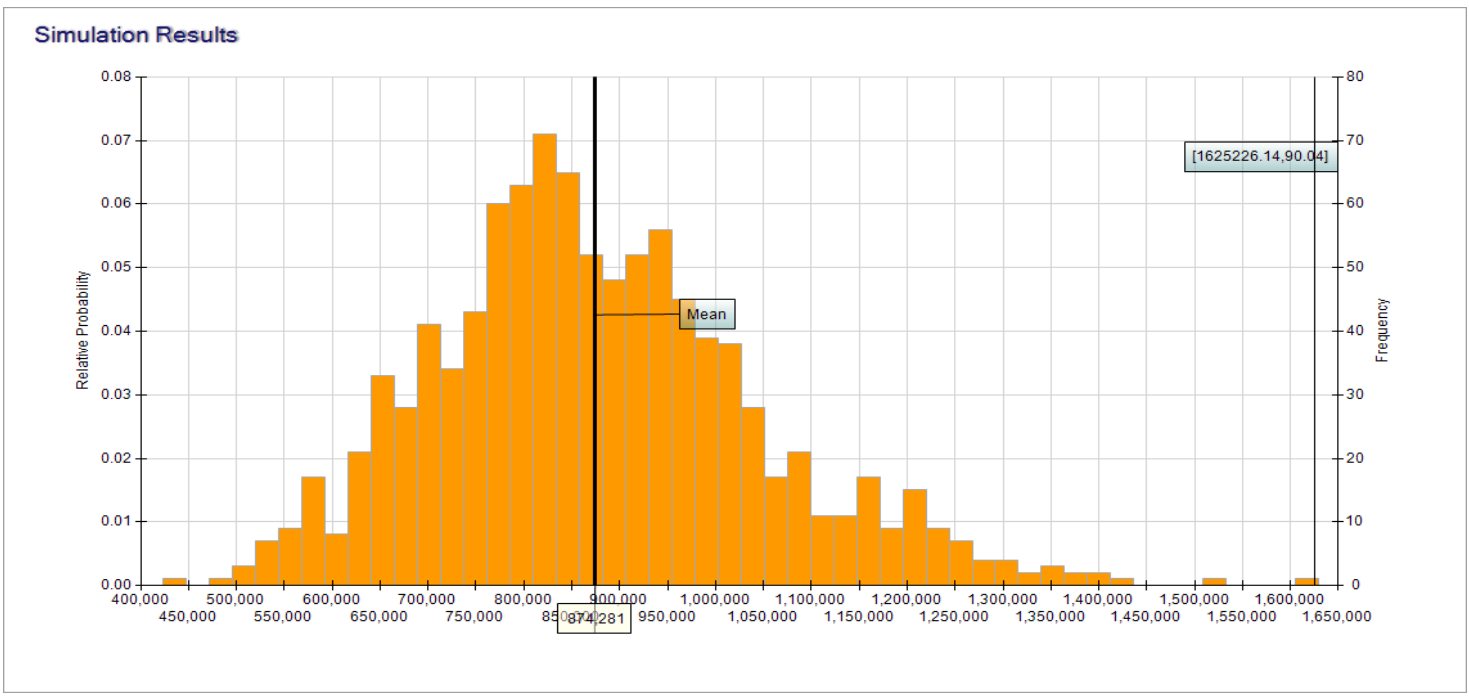

Figure 5.2: Probability Density Function of Total NPV

\subsubsection{Integrated MOdel FlowChart Description (OPTIMization Process)}

\subsubsection{Choosing Crude Price Forecasting Process (User Involved)}

The first step in the optimization process is to choose the price forecasting approach and to specify the price forecasting model under that approach. This section lists these models (descriptions are provided in the "Price Modeling" Chapter).

1. Deterministic Approach

a. The Flat Price Model where oil price is a fixed number for the life of the project.

b. The Floating Price Model where initial crude prices grow at a fixed annual growth rate.

2. Probabilistic Approach

a. Independent Lognormal Distribution: oil price is a lognormal distribution of a constant mean. Standard deviation is formulated to be monotonic with time to 
account for time as risk factor. Figure 5.21 shows 100 iterations of the ILD model prices.

b. Mean Reversion Model: Oil prices are variables that revert to an equilibrium price with time. This model is widely used in oil price forecasting processes. Figure 5.22 shows 50 iterations of the MRM prices.

c. Mean Reverting with Jumps: this process accounts of political and dramatic events by spikes or jumps in the price distribution; the spikes can be upward or downward.

d. Geometric Brownian Motion: The process combines a mean reversion model with Poisson distribution and is recommended by industry experts because it is practical and simple.

\subsubsection{Sampling Crude Oil Price Process}

This step is applicable for the probabilistic forecasting models. A simulation algorithm populates crude prices " $\mathrm{n}$ " iterations for the life of the project using a stochastic process. Each year has "n" price samples drawn from the probability distribution as shown in Figures 5.3 and 5.4 for independent lognormal and mean reverting processes, respectively. 


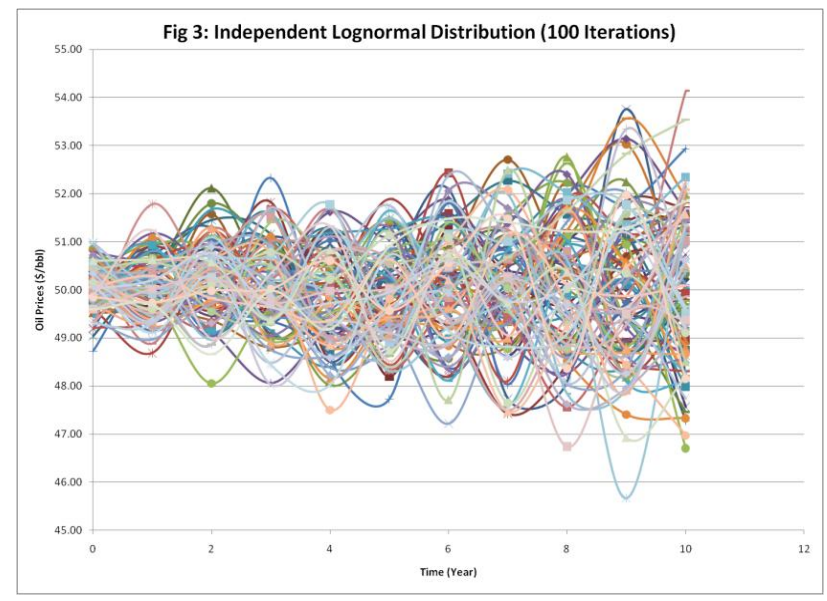

Figure 5.3: The ILD Price Model

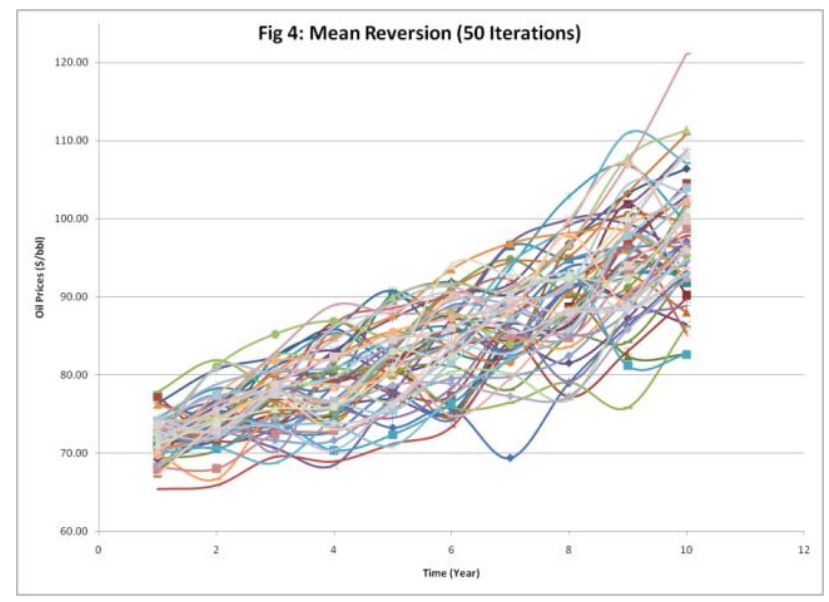

Figure 5.4: Mean Reversion Model

\subsubsection{Input Parameters and Data}

The basic parameters, which are required for field model calculations, are summarized in Table 5.2. (See the Parameters Description section for a description of the estimation of these parameters, and the Integrated Model Description section for a description of the implantation of these parameters). 
Table 5.2: $\quad$ Basic Parameters of the Fields

\begin{tabular}{|c|c|c|c|c|c|c|c|}
\hline Field & $\begin{array}{l}\text { Reserve } \\
\text { (Billion bbl) }\end{array}$ & $\begin{array}{l}\text { Depletion } \\
\text { Rate (\%) } \\
\end{array}$ & $\begin{array}{l}\text { Potential } \\
\text { (MBD) }\end{array}$ & $\begin{array}{l}\text { Minimum Operating } \\
\text { Rate (MBD) }\end{array}$ & $\begin{array}{l}\text { CAPEX } \\
\text { (Million \$) } \\
\end{array}$ & $\begin{array}{l}\text { Fixed OPEX } \\
\text { (Million \$) }\end{array}$ & $\begin{array}{c}\text { Variable OPEX } \\
(\$ / \mathrm{bbl})\end{array}$ \\
\hline 1 & 0.8 & $7 \%$ & 153 & 46 & 767 & 38.36 & 0.95 \\
\hline 2 & 4.0 & $3 \%$ & 329 & 99 & 2,630 & 131.51 & 0.95 \\
\hline 3 & 1.5 & $5 \%$ & 205 & 62 & 1,336 & 66.78 & 0.95 \\
\hline XX-Light Fields & 6.3 & & 688 & 206 & 4,733 & 236.64 & 0.95 \\
\hline 4 & 10.0 & $3.0 \%$ & 822 & 247 & 9,041 & 452.05 & 1.00 \\
\hline 5 & 5.0 & $4.0 \%$ & 548 & 164 & 4,384 & 219.18 & 1.20 \\
\hline 6 & 2.0 & $5.0 \%$ & 274 & 82 & 1,918 & 95.89 & 1.10 \\
\hline 7 & 2.5 & $5.5 \%$ & 377 & 113 & 2,637 & 131.85 & 1.20 \\
\hline Extra Light Fields & 19.5 & & 2,021 & 606 & 17,979 & 898.97 & 1.13 \\
\hline 8 & 1.0 & $4.0 \%$ & 110 & 33 & 767 & 38.36 & 1.30 \\
\hline 9 & 3.0 & $3.0 \%$ & 247 & 74 & 1,973 & 98.63 & 1.25 \\
\hline 10 & 5.0 & $2.5 \%$ & 342 & 103 & 2,911 & 145.55 & 1.40 \\
\hline 11 & 10.0 & $2.0 \%$ & 548 & 164 & 6,575 & 328.77 & 0.80 \\
\hline 12 & 15.0 & $2.0 \%$ & 822 & 247 & 11,507 & 575.34 & 0.70 \\
\hline 13 & 8.0 & $2.0 \%$ & 438 & 132 & 4,822 & 241.10 & 0.90 \\
\hline 14 & 4.0 & $4.0 \%$ & 438 & 132 & 3,507 & 175.34 & 1.20 \\
\hline 15 & 5.0 & $3.0 \%$ & 411 & 123 & 3,288 & 164.38 & 1.30 \\
\hline Light Fields & 51.0 & & 3,356 & 1,007 & 35,349 & $1,767.47$ & 1.11 \\
\hline 16 & 6.0 & $2.5 \%$ & 411 & 123 & 4,932 & 246.58 & 1.70 \\
\hline 17 & 15.0 & $2.5 \%$ & 1,027 & 308 & 17,466 & 873.29 & 1.50 \\
\hline 18 & 10.0 & $3.0 \%$ & 822 & 247 & 10,685 & 534.25 & 1.50 \\
\hline 19 & 10.0 & $3.0 \%$ & 822 & 247 & 10,685 & 534.25 & 1.40 \\
\hline Medium Fields & 41.0 & & 3,082 & 925 & 43,767 & $2,188.36$ & 1.53 \\
\hline 20 & 25.0 & $1.0 \%$ & 6885 & 205 & 17,123 & 856.16 & 1.50 \\
\hline 21 & 18.0 & $1.5 \%$ & 740 & 222 & 14,795 & 739.73 & 1.60 \\
\hline 22 & 12.0 & $2.0 \%$ & 658 & 197 & 11,836 & 591.78 & 2.00 \\
\hline 23 & 10.0 & $2.0 \%$ & 548 & 164 & 9,315 & 465.75 & 2.00 \\
\hline Heavy Fields & 65.0 & & 2,630 & 789 & 53,068 & $2,653.42$ & 1.78 \\
\hline
\end{tabular}

\subsubsection{Optimizing Process}

In the deterministic price forecasting approach, the calculated total NPV is a single value because each parameter is a single value and there is no probability distribution involved. As a result, the objective function of the optimization problem is the total NPV. The fact that total NPV is a single value simplifies the optimizing process. In the deterministic price forecasting approaches, the optimizing model maximizes the total NPV by modifying the fields' production rates allocation while meeting the imposed constraints. The optimization model calculates the production rate for each field, so that the total NPV is maximized. The optimization model was programmed to eliminate the 
effect of anticipatory error by assigning a single production rate for each field throughout the life of the field. In other words, a single production rate value is assigned to each field by the model to maximize the total NPV. Avoiding the anticipatory error is discussed in the Risk Analysis Workflow chapter in the Reveal Uncertain Variable section. (See Anticipatory Error section in the Technical Background chapter for a detailed description of the error).

On the other hand, the objective function (total NPV) is a probability density function in the case of probabilistic price forecasting because crude oil prices are a probability distribution. This adds to the complication of the optimization problem compared to the deterministic price modeling approach. Conventionally, the objective function is set to be the mean of the probability distribution of the performance attribute in the case of optimization under uncertainty, as suggested by Powell and Baker (2010). In the study, the mean of the total NPV is maximized by properly allocate (calculate) the production rates among the fields while meeting the constraints of the fields and facilities. The anticipatory error is eliminated by using the same technique as in the deterministic approach; moreover, the simulation error is minimized by using 1000 iterations in the Monte Carlo simulation process.

\subsection{Optimization Problem Formulation}

Each price forecasting approach (probabilistic or deterministic) requires a problem formulation resulting in two problem formulations. The main difference between the two formulations is the objective function; which is the sum of all the fields' NPVs in the case of the deterministic forecasting approach, and the mean of the distribution of the sum of all field's NPVs in the case of the probabilistic forecasting approach. Total NPV 
is a probability distribution because crude oil price forecasts are probability distributions in the probabilistic forecasting approach.

\subsubsection{Optimization Problem Formulation for the Deterministic Price FORECAST APPROACH}

In the deterministic approach, fields' production rates are re-allocated to maximize the "total NPV" while satisfying the model's constraints. There are two constraints imposed on fields' production rates; fields' maximum potential (upper bound), and facility minimum operating rate (lower bound). For example, production facility must maintain a minimum daily production rate order to operate economically. As a normal practice of reservoir engineers, studies to determine the maximum field's production rate to ensure sufficient sweeping and maintain reservoir pressure which is the

driving force for oil production. In the study, fields' depletion rates are used as the maximum allowed production rates. Depletion rates are function of reservoir studies (reservoir engineers' decision) and crude oil demand. The process of estimating fields' depletion rate values is discussed in model description section; the average depletion rate used in the study is $2 \%$.

In simple words, the model maximizes the total NPV of the company by reallocating fields' production rate subject to the followings:

- $\quad$ Field production rate has to be greater than facility minimum operating rate.

- $\quad$ Field production rate has to be less the field's potential.

- $\quad$ Total field production rate has to meet the daily target rate (functionality requirement) that is assigned by management. 


\subsubsection{Definition of Modeling Parameters}

Parameters used in the integrated optimization model are abbreviated in the formulation process section to simplify the equations. Here, these abbreviations are listed and defined.

$\mathrm{F}=$ Number of fields, $\mathrm{f}=1,2 \ldots 5$.

$C=$ Number of Crude Types, $c=1,2 \ldots C$ which defined as follows:

Classification of Crude Types

$\mathrm{C}=1(\mathrm{XX}-$ Light $)$

$\mathrm{C}=2$ (X-Light)

$\mathrm{C}=3$ (Light)

$\mathrm{C}=4$ (Medium)

$A R_{c}^{f}(t)=$ Annual Revenue for Field $f$ and Crude $c$ at time $t$

$P_{c}^{f}(t)=$ Oil Prices for Field $f$ and Crude $c$ at time $t$

$T=$ Number of Time Periods in the Study, $t=1,2 \ldots T$

$\operatorname{PT}_{c}^{f}(t)=$ Production Potential for Field $f$ and Crude $c$ at time $t$

$R_{c}^{f}=$ Original Reserve for Field $f$ and Crude $c$

$M R_{c}^{f}(t)=$ Minimum Operating Rate for Field $f$ and crude $c$ at tiem $t$

$\operatorname{CAP}_{c}^{f}(t)=$ Total Initial Capital Costs for Field $f$ and Crude $c$ at time $t$

FOP $_{c}^{f}(t)=$ Fixed Operating Costs for Field $f$ and Crude $c$ at time $t$

$\operatorname{VOC}_{c}^{f}(t)=$ Variable Operating Costs for Field $f$ and Crude $c$ at time $t$

$\operatorname{VOR}_{c}^{f}(t)=$ Variable Operating Rate for Field $f$ and Crude $c$ at time $t$

$\operatorname{TAX}(t)=$ Tax Rate at time $t$

$I=$ Discount Rate (To account for Time Value of Money.)

GR = Oil Pirce Annual Growth Rate

$\operatorname{TDR}(t)=$ Total Daily Desired Production Rate at Time $t$ 


\subsubsection{Modeling Decision Variables}

Decision variables are parameters that management modifies to maximize profit. In other words, decision variables are the parameters that management chooses to maximize profit. The model calculates the proper decision variables which are the fields' production rates to maximize the objective function (mean of total NPV distribution). Decision variable is defined as below in the formulations and calculations.

$$
\boldsymbol{P R}_{\boldsymbol{c}}^{f}(\boldsymbol{t})=\text { Production Rate for Field } f \text { and Crude } c \text { at time } t \text { (Constant). }
$$

\subsubsection{Optimization Model Calculations}

This section discusses the calculations used throughout the economicoptimization model. In the calculation part of the model, terms were dropped out of the calculation process due to the fact that the oil company is government owned. This impacts tax payments, royalties, net proceeds, interest payments, and principal payments. Moreover, the salvage term was dropped due to its negligible effect on the total NPV.

Equation 5.1 describes the calculation of the total NPV, which is the sum, over time and fields, of the discounted annual revenue minus fixed operating costs, variable operating costs, and capital costs. Equation 5.2 represents the annual revenue of a specific field at a specific time, which is the multiplication of annual production and crude oil price. Equation 5.3 is a deterministic price forecasting equation, which is used in the Floating Price forecasting model; the equation is used to convert the current price to future value using an annual price growth rate. The variable operating cost (OPEX) is calculated using Equation 5.4, for a given time period.

$$
\begin{gathered}
\text { NPV }=\text { Net Present Value } \\
\text { Total NPV }= \\
\sum_{t=0}^{t=T} \sum_{f=1}^{f=F} \sum_{c=1}^{c=C}\left[\left(A R_{c}^{f}(t)-F O P_{c}^{f}(t)-\operatorname{VOC}_{c}^{f}(t)-C A P_{c}^{f}(t)\right) /(1+I)^{t}\right]
\end{gathered}
$$

Where 


$$
\begin{aligned}
& A R_{c}^{f}(t)=\boldsymbol{P} \boldsymbol{R}_{c}^{f}(\boldsymbol{t}) * P_{c}^{f}(t) * 365 \\
& P_{c}^{f}(t)=P_{c}^{f}(t=0) *(1+G R)^{t} \\
& \operatorname{VOC}_{c}^{f}(t)=\boldsymbol{P} \boldsymbol{R}_{c}^{f}(\boldsymbol{t}) * \operatorname{VOR}_{c}^{f}(t)
\end{aligned}
$$

\subsubsection{Optimization Problem Formulation}

The optimization problem formulation consists of three parts: objective function, decision variables, and constraints. Optimization problems can be stated as follows: maximize the objective function by changing the decision variables subject to modeling constraints. This section presents the optimization statement of the optimization process that uses the deterministic price forecasting approach.

Maximize Total NPV

By Changing $\boldsymbol{P R}_{c}^{f}(\boldsymbol{t}) \forall f, c, t$

\section{Subject to}

$$
\begin{aligned}
& \boldsymbol{P} \boldsymbol{R}_{c}^{f}(\boldsymbol{t}) \leq P T_{c}^{f}(t) \forall f, c, t \\
& \boldsymbol{P} \boldsymbol{R}_{c}^{f}(\boldsymbol{t}) \geq M R_{c}^{f}(t) \forall f, c, t \\
& \sum_{\boldsymbol{f}=\mathbf{1}}^{\boldsymbol{f = F}} \sum_{\boldsymbol{c}=\boldsymbol{1}}^{\boldsymbol{c}=\boldsymbol{C}} \boldsymbol{P} \boldsymbol{R}_{\boldsymbol{c}}^{f}(\boldsymbol{t})=T D R(t) \forall t
\end{aligned}
$$

In summary, the optimization statement, using the deterministic price forecasting approach, is to maximize the total NPV by modifying the field production rates, subject to the following constraints:

1. Field production rate must be lower than the maximum field production potential.

2. Field production rate must be higher than the minimum facility operating level.

3. The company's total production rate must meet the target rate assigned by the management. 
During the optimization process, the optimization algorithm solves a distinct optimization problem (knapsack problem). The Knapsack problem is a combinatorial optimization problem. To illustrate the Knapsack problem, consider selecting items (each with given weight and value) from a set of given items so that the total value of the selected items are maximized while maintain the total weight of the items lower than the specified constraint. This Knapsack problem is solved in our optimization problem; the powerful algorithm, needed to solve a Knapsack problem, assigns all the fields at maximum potential (upper limit) starting from the highest value field. Then, the algorithm reduces the rates from the least value fields until the total target rate $\operatorname{TDR}(\mathrm{t})$ is met while the total NPV is maximized.

\subsubsection{Optimization Problem Formulation for the Probabilistic Price FORECAST APPROACH}

To account for the dynamics and volatility of crude oil prices, which impact the decision making process, stochastic processes were implemented to forecast the prices for the 5 crude grades over 10 years. Monte Carlo Simulation is used to program these stochastic processes. The optimization problem formulation for the probabilistic price forecasting approach is discussed here. The Independent Log-normal Distribution (ILD) price forecasting model is used in this section for illustration purposes.

In the probabilistic forecasting approach, field production rates are re-allocated to maximize the mean of total NPV distribution while satisfying the model's constraints. There are two constraints imposed on field production rates; field maximum potentials (upper bound), and facility minimum operating rate (lower bound). For example, a production facility must maintain a minimum daily production rate in order to operate economically. As a normal practice, reservoir engineers perform studies to determine the 
maximum allowable field production rate while ensuring sufficient oil sweep in the reservoir and maintaining reservoir pressure, which is the driving force for oil production. In the study, field depletion rates are used as the maximum allowed production rates to include the reservoir engineers' requirements in the study. Depletion rate is a function of reservoir studies (decisions of reservoir engineers) and crude oil demand. The process of estimating field depletion rate values is discussed in the Model Description section; the average depletion rate used in the study is $2 \%$.

Put simply, the model maximizes the mean of the total NPV distribution of the company by re-allocating fields' production rate subject to the followings:

- $\quad$ Field production rate has to be greater than facility minimum operating rate.

- $\quad$ Field production rate has to be less the field's potential.

- $\quad$ Total field production rate has to meet the daily target rate (functionality requirement) that is assigned by management.

\subsubsection{Definition of Modeling Parameters}

Parameters used in the integrated optimization model are abbreviated in the formulation process section to simplify the equations. Here, these abbreviations are listed and defined.

$$
\begin{aligned}
& \text { NPV }=\text { Net Present Value } \\
& \begin{aligned}
\mathrm{F}=\text { Number of fields, } \mathrm{f}=1,2 \ldots 5 . \\
\mathrm{C}=\text { Number of Crude Type, } \mathrm{c}=1,2 \ldots \mathrm{C} \text { which defined as follow: } \\
\text { Crude Types Classification } \\
\mathrm{c}=1 \text { (XX- Light) } \\
\mathrm{c}=2 \text { (Extra Light) }
\end{aligned}
\end{aligned}
$$




$$
\begin{aligned}
& \mathrm{c}=3 \text { (Light) } \\
& \mathrm{c}=4 \text { (Medium) } \\
& \mathrm{c}=5 \text { (Heavy) } \\
& \mathrm{AR}_{\mathrm{i}, \mathrm{c}}^{\mathrm{f}}(\mathrm{t})=\text { Iteration } \mathrm{i} \text { of Annual Revenue for Field } \mathrm{f} \text { and crude } \mathrm{c} \text { at time } \mathrm{t} \text {. } \\
& \mathrm{T}=\text { Number of Time Periods in the Study, } \mathrm{t}=1,2 \ldots \mathrm{T} \\
& \mathrm{PT}_{\mathrm{c}}^{\mathrm{f}}(\mathrm{t})=\text { Production Potential for Field } \mathrm{f} \text { and Crude } \mathrm{c} \text { at time } \mathrm{t} \text {. } \\
& \mathrm{R}_{\mathrm{c}}^{\mathrm{f}}=\text { Original Reserve for Field } \mathrm{f} \text { and Crude } \mathrm{c} \text {. } \\
& \mathrm{MR}_{\mathrm{c}}^{\mathrm{f}}(\mathrm{t})=\text { Minimum Operating Rate for Field } \mathrm{f} \text { and crude } \mathrm{c} \text { at tiem } \mathrm{t} \\
& \operatorname{CAP}_{\mathrm{c}}^{\mathrm{f}}(\mathrm{t})=\text { Total Initial Capital Costs for Field } \mathrm{f} \text { and Crude } \mathrm{c} \text { at time } \mathrm{t} \text {. } \\
& \text { FOP }_{c}^{f}(t)=\text { Fixed Operating Costs for Field } f \text { and Crude } c \text { at time } t \text {. } \\
& \operatorname{VOC}_{\mathrm{c}}^{\mathrm{f}}(\mathrm{t})=\text { Variable Operating Costs for Field } \mathrm{f} \text { and Crude } \mathrm{c} \text { at time } \mathrm{t} \text {. } \\
& \operatorname{VOR}_{\mathrm{c}}^{\mathrm{f}}(\mathrm{t})=\text { Variable Operating Rate for Field } \mathrm{f} \text { and Crude } \mathrm{c} \text { at time } \mathrm{t} \text {. } \\
& \operatorname{TAX}(\mathrm{t})=\text { Tax Rate at time } \mathrm{t} \text {. } \\
& \mathrm{I}=\text { Discount Rate (To account for Time Value of Money. ) } \\
& \operatorname{TDR}(\mathrm{t})=\text { Total Daily Desired Production Rate at Time } \mathrm{t} \text {. }
\end{aligned}
$$

\subsubsection{Modeling Decision Variables}

Decision variables are parameters that managements modify to maximize profit. In other words, decision variables are the parameters that managements choose to maximize profit. The model calculates the proper decision variables which are the field production rates to maximize the objective function (mean of total NPV distribution). Decision variable is defined as below in the formulations and calculations.

$$
\boldsymbol{P R}_{\boldsymbol{c}}^{f}(\boldsymbol{t})=\text { Production Rate for Field } f \text { and Crude } c \text { at time } t \text { (Constant). }
$$




\subsubsection{Optimization Model Calculations}

This section discusses the calculations used throughout the economicoptimization model. In the calculation part of the model, terms were dropped from the calculation process because of the assumption that the company is government owned; these terms include tax payments, royalties, net proceeds, interest payments, and principal payments. Moreover, the salvage term was dropped due to its negligible effect on the total NPV.

Equation 5.8 describes the calculation of the total NPV, which is the sum, over time and fields, of the discounted annual revenue minus the discounted total cost. The total cost consists of fixed operating costs, variable operating costs, and capital costs. Equation 5.9 represents the annual revenue of a specific field at a specific time, which is the multiplication of annual production and crude oil price. The variable operating cost (OPEX) is calculated using Equation 5.10, for a given time period.

$$
\begin{gathered}
\text { NPV }=\text { Net Present Value. } \\
\text { Total NPV }= \\
\sum_{\mathrm{t}=0}^{\mathrm{t}=\mathrm{T}} \sum_{\mathrm{f}=1}^{\mathrm{f}=\mathrm{F}} \sum_{\mathrm{c}=1}^{\mathrm{c}=\mathrm{C}}\left[\left(\mathrm{AR}_{\mathrm{c}}^{\mathrm{f}}(\mathrm{t})-\mathrm{FOP}_{\mathrm{c}}^{\mathrm{f}}(\mathrm{t})-\operatorname{VOC}_{\mathrm{c}}^{\mathrm{f}}(\mathrm{t})-\mathrm{CAP}_{\mathrm{c}}^{\mathrm{f}}(\mathrm{t})\right) /(1+\mathrm{I})^{\mathrm{t}}\right]
\end{gathered}
$$

Where

$$
\begin{aligned}
& \operatorname{AR}_{\mathrm{c}}^{\mathrm{f}}(\mathrm{t})=P \boldsymbol{R}_{c}^{f}(\mathrm{t}) * \mathrm{P}_{\mathrm{c}}^{\mathrm{f}}(\mathrm{t}) * 365 \\
& \operatorname{VOC}_{\mathrm{c}}^{\mathrm{f}}(\mathrm{t})=P R_{c}^{f}(t) * \operatorname{VOR}_{\mathrm{c}}^{\mathrm{f}}(\mathrm{t})
\end{aligned}
$$

\subsubsection{Optimization Problem Formulation}

The optimization problem formulation consists of three parts: objective function, decision variables, and constraints. Optimization problems are defined as follow: to maximize the objective function by changing the decision variables subject to modeling constraints. This section presents the optimization statement of the optimization process that uses the probabilistic price forecasting approach. 
Maximize $\quad$ E[total NPV]

By Changing $P R_{c}^{f}(t) \forall \mathrm{f}, \mathrm{c}, \mathrm{t}$

Subject to

$$
\begin{aligned}
& P R_{c}^{f}(t) \leq \mathrm{PT}_{\mathrm{c}}^{\mathrm{f}}(\mathrm{t}) \forall \mathrm{f}, \mathrm{c}, \mathrm{t} \\
& P \boldsymbol{R}_{c}^{f}(\mathrm{t}) \geq \mathrm{MR}_{\mathrm{c}}^{\mathrm{f}}(\mathrm{t}) \forall \mathrm{f}, \mathrm{c}, \mathrm{t} \\
& \sum_{\mathrm{f}=1}^{\mathrm{f}=\mathrm{F}} \sum_{\mathrm{c}=1}^{\mathrm{c}=\mathrm{C}} \boldsymbol{P} \boldsymbol{R}_{c}^{f}(\mathrm{t})=\mathrm{TDR}(\mathrm{t}) \forall \mathrm{t}
\end{aligned}
$$

In summary, the optimization statement for the probabilistic price forecasting approach is to maximize the mean of total NPV distribution by modifying field production rates, subject to the following constraints:

1. Field production rate must be lower the maximum field production potential.

2. Field production rate must be higher than the minimum facility operating rate.

3. The company's total production rate must meet the target rate assigned by the management.

Similar to the deterministic pricing approach, the Knapsack problem is solved during the optimization process in the probabilistic forecasting approach. All the fields are assigned at the maximum potential (upper limit) starting from the highest value field by the greedy algorithm. Then, the algorithm reduces the rates from the least value fields until the total target rate $\operatorname{TDR}(\mathrm{t})$ is met while the mean of the total NPV distribution is maximized.

\subsection{Integrated Model Description}

Optimization models, simulation models, and stochastic process models have been built and integrated using the Risk Solver Platform (RSP) (EXCEL add-in 
software). RSP is the main tool used in the study for optimization, simulation and stochastic processes. RSP is fast solving software, which handles optimization, simulation, probability distribution generation, etc. This section describes the economics model, the optimization model, and the simulation model for both deterministic and probabilistic approaches. Crude price models are discussed in the Price Modeling section.

Using the Risk Solver Platform, the optimization algorithm was embedded into the economic evaluation spreadsheet to maximize the total NPV or mean of total NPV. The integrated model (Excel spreadsheet) was divided into three modules, parameters, calculations, and input \& output.

\subsubsection{InTEgrated Model Modules}

The parameters module was placed in a separate sheet named Parameter. The calculations module was divided by the number of fields so that each field's calculations were placed in a separate sheet. The last module Input \& Output, where most of the interactions take place, was placed in a separate sheet. Powell and Baker (2010) recommended separating modules in the process of building decision support models to enhance the clarity and reduce the complexity of the whole model.

\subsubsection{Integrated Model Parameters Module}

The Parameters module is divided into two sections. The first section, deduction rates, is shown in Table 5.3. The table reveals the assumption that the company is government owned, so there are no tax or royalty payments. On the other hand, it is assumed that the company imposes a $15 \%$ discount rate on the economic evaluation of oil projects. Discount rate is used in the discounting process, which moves cash flow from future time periods back to the present time, so cash flow at different periods can be

compared (Jablonowski, 2009). Without discounting, cash flow at different periods of 
time cannot be compared because of time value of money (Jablonowski, 2009), which states that a dollar today is more valuable than a dollar sometime in the future (Hartman, 2007). The difference in the dollar value is due to the value of an investment; for example, the money can be invested in a bank account with a specific rate of return rather than the oil projects (Jablonowski, 2009 and Hartman, 2007). In addition to the time value of money (investment value), discounting incorporates the risk of finding commercially producible hydrocarbon. In short, the discounting process is a decision criterion that an oil project must at least return the discount rate value to be acceptable (Jablonowski, 2009).

Table 5.3: $\quad$ Deduction Rates

\begin{tabular}{|ll|}
\hline Tax rate & $0 \%$ \\
Discount Rate & $15 \%$ \\
Royalty & $0 \%$ \\
\hline
\end{tabular}

The second part of the parameter module is the field parameters, which are necessary for cash flow calculations. These include crude type, reserve, depletion rate, maximum production potential, facility minimum operating rate, capital expenditure (CAPEX), and fixed and variable operating expenditure (OPEX). Table 5.4 lists Field 1 parameters as an example.

Table 5.4: $\quad$ Field 1 Parameters 


\begin{tabular}{|lc|}
\hline Field & 1 \\
Crude Type & $\mathrm{XXI}$ \\
Reserves (Billion bbl) & 0.8 \\
Maximum Allowed Depletion Rate (\%) & $7.0 \%$ \\
Production Potential (MBD) & 153 \\
Facility Minimum Rate (MBD) & 46 \\
CAPEX (Million \$) & 0 \\
OPEX, Fixed Cost (Million \$/Yr) & 38 \\
OPEX, Variable Cost (\$/bbI) & 0.95 \\
Decline rate of oil production & $0 \%$ \\
Life of Capital (Yrs) & 10 \\
\hline
\end{tabular}

The estimation processes of these parameters are significantly impacted by the assumptions used. Table 5.5 lists these assumptions, which were specified by industry experts. The table reveals the fact that most of the parameters are functions of crude grade; reserves range is the range of reserves from which the field reserves are specified. The reserve ranges are classified by crude grade; this classification reveals an increasing trend in the reserve as the crude grade decreases. The increasing oil reserves as the crude grade decreases reflects the fact that most of the Light crude, easy oil, has been produced and there is more of the Heavy crude in the ground. Lighter crudes are cheaper to produce and sell at higher prices, so it is preferred to produce them at higher rates than heavier crudes, which are more expensive to produce and treat, and are sold at lower prices. The reserve estimation was randomly specified within the range of the crude grade that the field belongs to; for example, the reserves of the three XXL fields were randomly specified to be between 0.1 and 5 billion barrels of oil as shown in Table 5.38 .

Depletion rate is the rate, out of original reserve, at which an oil reserve is being depleted annually. In practice, management makes the decision on the value of the depletion rate. This management strategy is dictated by demand, reservoir property studies, and politics. Reservoir studies are conducted to determine the maximum allowable production rate for a specific field while maintaining efficient oil sweep. Political events and demand changes within a specific crude grade play a major role in 
deciding the production rate of a specific crude grade and/or specific field. In the study, the depletion rate estimation process was dictated by crude grade and reserve size; large Heavy crude reservoirs are depleted at lower rates than small lighter reservoirs. Lighter crude reserves are smaller and in high demand and high supply volumes. As mentioned earlier, lighter crude has been produced over time due to its relatively low production costs and high sales prices; this fact leads to the current low Light crude reserves. The high demand for the Light crude comes from the fact that the Light crude holds higher energy than the Heavy, while the higher supply is due to its low production costs and higher sales price. The production potential is the maximum production rate a field can produce, which is the annual depletion rate converted to the daily rate. Production potential is the upper bound of the field production rate in the optimization model.

Minimum operating rate is the lower bound in the optimization model which is assumed to be $30 \%$ of the production potential. Minimum operating rate is the minimum rate that should be maintained by the facility to assure economical and safe operation.

Capital expenditure (CAPEX) is applicable for development or incremental project. Similar to depletion rate, CAPEX estimation was dictated by crude grade and reserve size. "Fixed Operating Expenditure (Fixed OPEX)" was assumed to be 5\% of CAPEX. "Variable Operating Expenditure (Variable OPEX)" estimation was mainly dictated by crude grade; lower variable OPEX was imposed on lighter crudes due to it lower production costs. Fixed OPEX cost is applicable whenever the field is on production regardless of the rate. On the other hand, variable OPEX cost is a function of production rate, so it's applicable only if the field is producing. It is assumed that a fixed production rates are maintained throughout the life of the project; the costs to maintain fixed production rate are incorporated in variable OPEX costs. Table 5.6 lists the fields' 
parameters required for calculations. Price forecasting parameters are presented and discussed in price modeling section.

Table 5.5: Field Parameter Assumptions

\begin{tabular}{|lcccccc|}
\hline Crude Grade & $\begin{array}{c}\text { Reserves Range } \\
\text { MMBBI }\end{array}$ & $\begin{array}{c}\text { Depletion Rate Range } \\
\text { \% }\end{array}$ & $\begin{array}{c}\text { Min Operating Rate } \\
\text { (\% of Depletion) }\end{array}$ & $\begin{array}{c}\text { CAPEX } \\
\text { M\$̦/bbl Required }\end{array}$ & $\begin{array}{c}\text { Fixed OPEX } \\
\text { (\% of CAPEX) }\end{array}$ & $\begin{array}{c}\text { Variable OPEX } \\
\text { (\$⿳/口bl) }\end{array}$ \\
\hline \hline XX-Light & $100-5,000$ & $2-8$ & 30 & $5,000-10,000$ & 5 & $0.3-1.0$ \\
X-Light & $200-10,000$ & $2-6$ & 30 & $6,000-12,000$ & 5 & $0.5-1.2$ \\
Light & $500-15,000$ & $2-4$ & 30 & $7,000-14,000$ & 5 & $0.7-1.4$ \\
Medium & $1,000-20,000$ & $1-3$ & 30 & $10,000-20,000$ & 5 & $1.0-1.7$ \\
Heavy & $2,000-25,000$ & $1-2$ & 30 & $15,000-25,000$ & 5 & $1.2-2.0$ \\
\hline
\end{tabular}

Table 5.6: Field Parameters

\begin{tabular}{|c|c|c|c|c|c|c|c|}
\hline Field & $\begin{array}{c}\text { Reserve } \\
\text { (Billion bbl) }\end{array}$ & $\begin{array}{l}\text { Depletion } \\
\text { Rate (\%) }\end{array}$ & $\begin{array}{l}\text { Potential } \\
\text { (MBD) }\end{array}$ & $\begin{array}{l}\text { Minimum Operating } \\
\text { Rate (MBD) }\end{array}$ & $\begin{array}{c}\text { CAPEX } \\
\text { (Million \$) }\end{array}$ & $\begin{array}{l}\text { Fixed OPEX } \\
\text { (Million \$) }\end{array}$ & $\begin{array}{c}\text { Variable OPEX } \\
\text { (\$/bbl) }\end{array}$ \\
\hline 1 & 0.8 & $7 \%$ & 153 & 46 & 767 & 38.36 & 0.95 \\
\hline 2 & 4.0 & $3 \%$ & 329 & 99 & 2,630 & 131.51 & 0.95 \\
\hline 3 & 1.5 & $5 \%$ & 205 & 62 & 1,336 & 66.78 & 0.95 \\
\hline XX-Light Fields & 6.3 & & 688 & 206 & 4,733 & 236.64 & 0.95 \\
\hline 4 & 10.0 & $3.0 \%$ & 822 & 247 & 9,041 & 452.05 & 1.00 \\
\hline 5 & 5.0 & $4.0 \%$ & 548 & 164 & 4,384 & 219.18 & 1.20 \\
\hline 6 & 2.0 & $5.0 \%$ & 274 & 82 & 1,918 & 95.89 & 1.10 \\
\hline 7 & 2.5 & $5.5 \%$ & 377 & 113 & 2,637 & 131.85 & 1.20 \\
\hline Extra Light Fields & 19.5 & & 2,021 & 606 & 17,979 & 898.97 & 1.13 \\
\hline 8 & 1.0 & $4.0 \%$ & 110 & 33 & 767 & 38.36 & 1.30 \\
\hline 9 & 3.0 & $3.0 \%$ & 247 & 74 & 1,973 & 98.63 & 1.25 \\
\hline 10 & 5.0 & $2.5 \%$ & 342 & 103 & 2,911 & 145.55 & 1.40 \\
\hline 11 & 10.0 & $2.0 \%$ & 548 & 164 & 6,575 & 328.77 & 0.80 \\
\hline 12 & 15.0 & $2.0 \%$ & 822 & 247 & 11,507 & 575.34 & 0.70 \\
\hline 13 & 8.0 & $2.0 \%$ & 438 & 132 & 4,822 & 241.10 & 0.90 \\
\hline 14 & 4.0 & $4.0 \%$ & 438 & 132 & 3,507 & 175.34 & 1.20 \\
\hline 15 & 5.0 & $3.0 \%$ & 411 & 123 & 3,288 & 164.38 & 1.30 \\
\hline Light Fields & 51.0 & & 3,356 & 1,007 & 35,349 & $1,767.47$ & 1.11 \\
\hline 16 & 6.0 & $2.5 \%$ & 411 & 123 & 4,932 & 246.58 & 1.70 \\
\hline 17 & 15.0 & $2.5 \%$ & 1,027 & 308 & 17,466 & 873.29 & 1.50 \\
\hline 18 & 10.0 & $3.0 \%$ & 822 & 247 & 10,685 & 534.25 & 1.50 \\
\hline 19 & 10.0 & $3.0 \%$ & 822 & 247 & 10,685 & 534.25 & 1.40 \\
\hline Medium Fields & 41.0 & & 3,082 & 925 & 43,767 & $2,188.36$ & 1.53 \\
\hline 20 & 25.0 & $1.0 \%$ & 685 & 205 & 17,123 & 856.16 & 1.50 \\
\hline 21 & 18.0 & $1.5 \%$ & 740 & 222 & 14,795 & 739.73 & 1.60 \\
\hline 22 & 12.0 & $2.0 \%$ & 658 & 197 & 11,836 & 591.78 & 2.00 \\
\hline 23 & 10.0 & $2.0 \%$ & 548 & 164 & 9,315 & 465.75 & 2.00 \\
\hline Heavy Fields & 65.0 & & 2,630 & 789 & 53,068 & $2,653.42$ & 1.78 \\
\hline
\end{tabular}




\subsubsection{Cash Flow Calculations Module}

A field cash flow calculation involves complex calculations. To reduce the complexity of this module, each field's cash flow calculations were performed in a separate sheet. Each field's calculation sheet is divided into six sub-modules which are oil prices, production, deductions, taxes, non-taxes items and net cash flow \& NPV calculations. This section discusses these sub-modules.

\subsection{Crude Oil Price Forecasting Sub-Module}

Prices were forecasted in this study using deterministic and probabilistic approaches. This sub-modules presents the price forecasting models for both approaches. Due to the importance and complexity of these price forecasting models, they are discussed in detail in the Crude Oil Price Modeling section.

\subsection{Oil Production Sub-Module}

Production sub-module calculates field daily and yearly oil production, yearly revenues, cumulative production, and remaining reserves for each year.

Table 5.7 shows a sample of oil production sub-module. Field daily average productions, which are the decision variables for the optimization process, are supplied to the production sub-module by the optimizer. Yearly production is calculated using Equation 5.14. Yearly production and crude price generate field yearly revenue. Yearly cumulative production and remaining reserve are tracked to prevent cases of overproduction.

$$
\text { Annual Production }=\text { Daily Production } * 365
$$

Table 5.7: $\quad$ Oil Production Sub-Module 


\begin{tabular}{|c|c|c|c|c|c|c|}
\hline Time & Yrs & 0 & 1 & 2 & 3 & 4 \\
\hline \multicolumn{7}{|l|}{ Production } \\
\hline Average Production & $\mathrm{mbbl} / \mathrm{day}$ & & & 548 & 548 & 548 \\
\hline Year End Production Rate & $\mathrm{mbbl} / \mathrm{day}$ & & & & & \\
\hline Yearly Produciton & $(\mathrm{mbbl})$ & & & 200,000 & 200,000 & 200,000 \\
\hline Royality & (mbbl) & 0 & 0 & 0 & 0 & 0 \\
\hline Net Production & (mbbl) & & & 200,000 & 200,000 & 200,000 \\
\hline Net Revenues & \$ million & 0 & 0 & 16,730 & 16,897 & 17,066 \\
\hline Cummulative Production & $\mathrm{mbbl}$ & & & 200,000 & 400,000 & 600,000 \\
\hline Remaining Reserves & $\mathrm{mmbbl}$ & & & 600 & 400 & 200 \\
\hline
\end{tabular}

\subsection{Deductions Sub-Module}

Deductions are the negative cash flows in the cash flow model; these negative cash flow items are tax deductible. In other words, these negative cash flow items are subtracted before applying the tax to the remaining amount. Deductions include fixed and variable operating costs, depreciation, depletion and intangible drilling costs. Although the study neglects tax payments, the model was designed to handle taxes in the deductions module. Fixed operating cost is a fixed cost to operate the field that will be deducted once the field is in production regardless of the rate. On the other hand, variable operating cost is a multiple of the production rate and is a function of reservoir properties, logistics and market conditions. Depreciation and depletion are tax deductible items that do not affect the calculations. The concept behind depreciation is that the facilities are wearing out and decreasing in value, so the oil company receives a tax discount equivalent to the reduction in the facilities' values. Intangible drilling costs include intangible drilling fluid (mud) cost, rental equipment cost, insurance, etc. For simplicity, intangible drilling costs are incorporated into variable operating costs. Table 5.8 is a sample of a deductions sub-module.

Table 5.8: Deduction Sub-Module 


\begin{tabular}{|lllllllll|}
\hline Time (Yrs) & & & 0 & 1 & 2 & 3 & 4 & 5 \\
\hline Deductions & & & & & & & \\
\hline Production Taxes & & 0 & 0 & 0 & 0 & 0 & 0 \\
OPEX & Fixed & \$million & 0 & 0 & 38 & 38 & 38 & 38 \\
& Variable & \$million & 0 & & 53 & 53 & 53 & 53 \\
Intangilbe Drilling Costs & & & 0 & 0 & 0 & 0 & 0 & 0 \\
Depreciation 1 & Depreciable Value & \$million & 0 & 0 & 0 & 0 & 0 & 0 \\
& Dt & \$million & & & 0 & 0 & 0 & 0 \\
Depreciation 2 & Depreciable Value & \$million & & 0 & 0 & 0 & 0 & 0 \\
& Dt & \$million & & & 0 & 0 & 0 & 0 \\
Depletion & Cost Basis & \$million & & & 0 & 0 & 0 & 0 \\
Amortization & Dt & \$million & 0 & & 0 & 0 & 0 & 0 \\
Interest Payments & & & 0 & 0 & 0 & 0 & 0 & 0 \\
\hline
\end{tabular}

\subsection{Tax Payments Sub-Module}

This sub-module does not affect the calculations because of the assumption that the company is government owned and does not pay tax. This module was built to equip the integrated model with the capability of handling tax payments.

\subsection{Non-Tax Items Sub-Module}

Non-tax items are the positive and negative cash flows that do not impact tax payments. In other words, non tax items are not tax deductible, and include capital expenditures, salvage value, net proceeds and principal payments on a loan.

Capital expenditure (CAPEX) is the initial development cost. The study handles existing fields that do not incorporate CAPEX cost; CPEX does not impact the cash flow calculations. Salvage value was not considered in the model due to its negligible impact on the total NPV and final output. The company does not consider a financing option due to the assumption that it is a national oil company (NOC). As a result, net proceeds and principal payments are not considered in the calculations.

\subsection{Net Cash Flow and NPV Calculations Sub-Module}

This section handles net cash flow (NCF), discounted net cash flow (DNCF) and net present value (NPV) calculations. 
(NCF) was calculated by summing up positive and negative cash flows for each year. Then, NCF was discounted to the present value, using Equation 5.15, resulting in (DNCF). Figures 5.5 and 5.6 show cash flow diagrams for development and producing field projects, respectively. The final output of the calculation module is the NPV of each field, which is the sum of all the DNCF. The objective function in optimization model is the sum of all field NPVs in the deterministic price forecasting approach and the Expected Value of the sum of field NPVs in the probabilistic approach.

$$
\text { Present Value }=\frac{\text { Future Value }}{(1+\text { Discount Rate })^{\text {Time }}}
$$

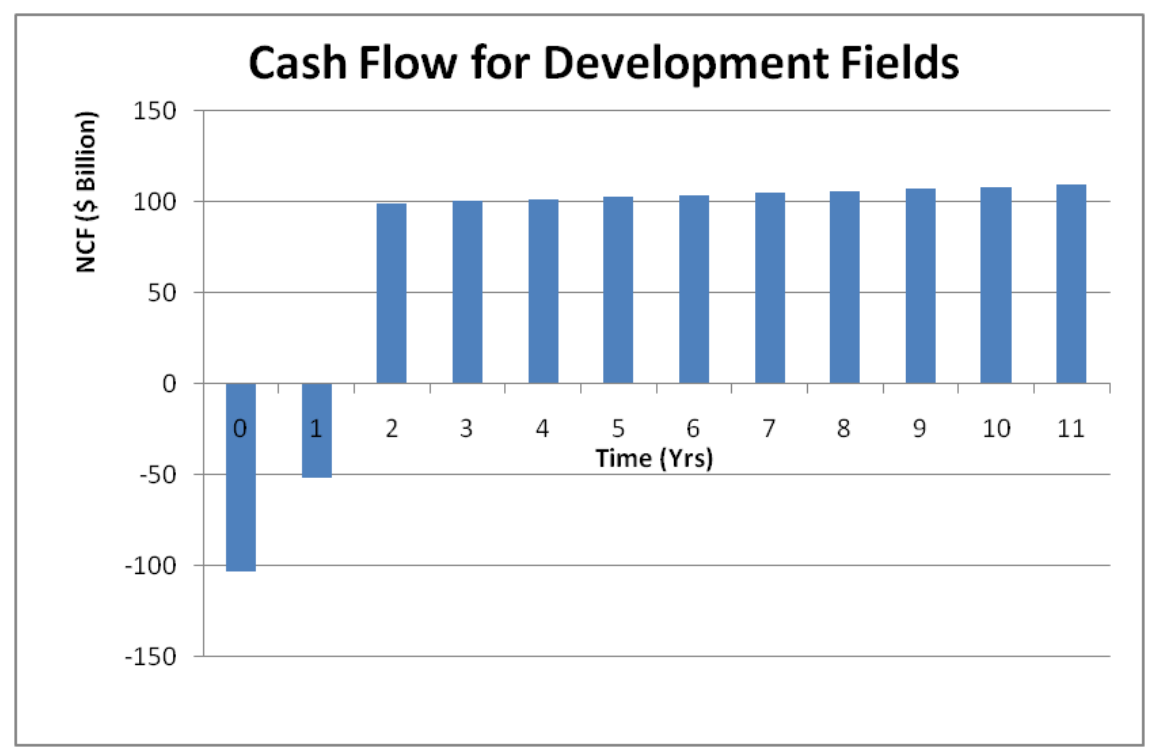

Figure 5.5: Cash Flow Diagram of Development Field Projects 


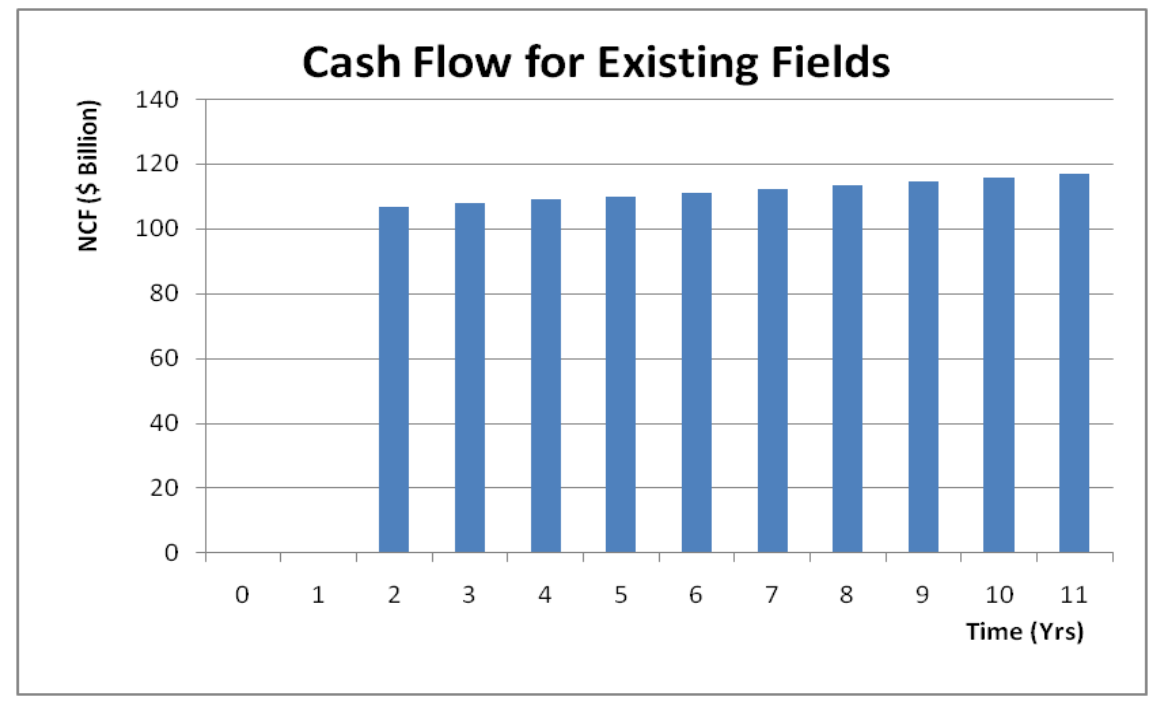

Figure 5.6: Cash Flow Diagram of Producing Field Project

\subsubsection{Input \& Output Module}

The input \& Output module is the last and most important module where most of the user-model interactions take place. This module includes users' input, final output of the integrated model, and optimization problem coding. The module is divided into four sub-modules: inputs, objective function, decision variables (output), and constraints submodules.

\subsection{Users' Input Sub-Module}

The input sub-module is different for each price forecasting model. This submodule consists of two parts; first, the company's total production target rate. The second part is the parameters required for the price forecasting model. The second part is where the differences between the price forecasting models occur. Tables 5.9- 5.14 present the input sub-module for the Flat Price model, Floating Price model, ILD model, GBM model, MRM model, and MRJM model, respectively.

Table 5.9: Input Sub-Module for the Flat Price Forecasting Model 


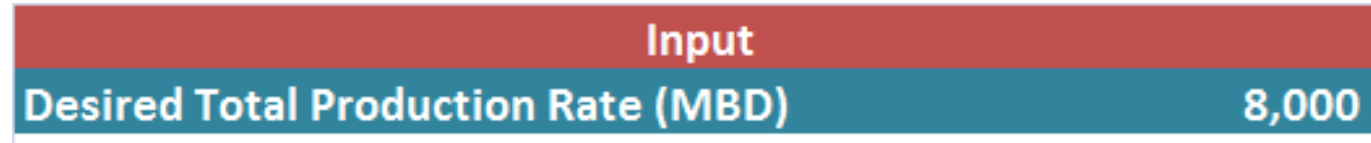

\begin{tabular}{lc|}
\multicolumn{2}{c}{ Initial Oil Prices } \\
\hline Crude Type & Initial Oil Prices (\$/bbl) \\
\hline XXL & 64.14 \\
XI & 62.16 \\
L & 60.36 \\
M & 57.99 \\
H & 56.23 \\
\hline
\end{tabular}

Table 5.10: Input Sub-Module for the Floating Price Forecasting Model

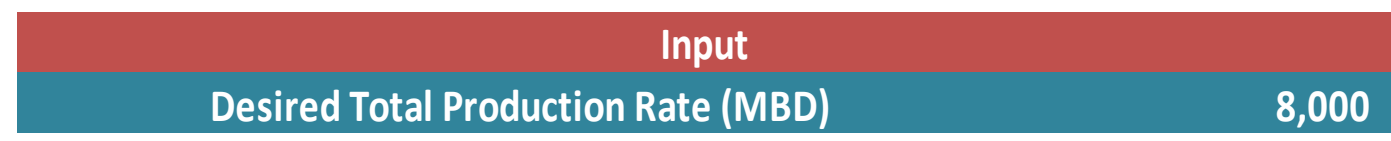

\begin{tabular}{|c|c|c|c|c|}
\hline \multicolumn{5}{|c|}{ Initial Oil Prices \& Growth Rate } \\
\hline \multicolumn{5}{|c|}{ Oil Prices at Time 0 and Growth Rate } \\
\hline & Crude Type & & $\$ / \mathrm{bbl})$ & Oil Prices Growth Rate (\%) \\
\hline$X X-L$ & & $\$$ & 44.06 & $1 \%$ \\
\hline$X-\mathrm{L}$ & & $\$$ & 42.69 & $1 \%$ \\
\hline$A L$ & & $\$$ & 34.41 & $1 \%$ \\
\hline AM & & $\$$ & 33.36 & $1 \%$ \\
\hline $\mathrm{AH}$ & & $\$$ & 32.63 & $1 \%$ \\
\hline
\end{tabular}

Table 5.11: Input Sub-Module for the Independent Log-normal Distribution Price Forecasting Model 

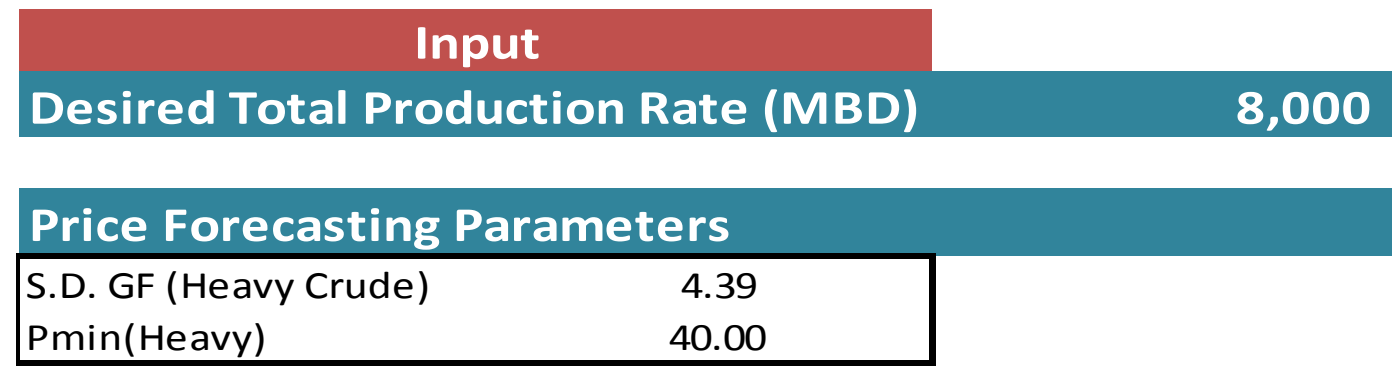

\begin{tabular}{|lcc|}
\hline \multicolumn{1}{c}{ Crude } & Mean Price (\$) & Initial S.D. \\
\cline { 2 - 3 } Heavy & 56.23 & 26.06 \\
Medium & 57.99 & 26.08 \\
Light & 60.36 & 26.11 \\
X-Light & 62.16 & 26.33 \\
XX-Light & 64.14 & 26.64 \\
\hline
\end{tabular}

Table 5.12: Input Sub-Module for the Geometric Brownian Motion Price Forecasting Model 


\section{Input \\ Desired Total Production Rate (MBD)}

\section{8,000}

\section{Crude Price Forecasting Parameters}

\begin{tabular}{|lcl|}
\hline \multicolumn{1}{|c|}{ Parameter } & Value & \multicolumn{1}{c|}{ Remarks } \\
\hline \hline$\alpha$ (Heavy Crude Drift) & 0.23 & Drift \\
$P^{*}$ (Heavy Crude Long-Run Mean Price) & 56.23 & Long run average Price \\
$\sigma$ (Heavy Volatility) & 0.42 & Volatility \\
Pmin(Heavy) & 40.00 & Annual (2004-2010) \\
\hline
\end{tabular}

\begin{tabular}{|c|c|c|}
\hline Crude Grade & Mean Price (\$) & Initial S.D. \\
\hline Heavy & 56.23 & 26.06 \\
\hline Medium & 57.99 & 26.08 \\
\hline Light & 60.36 & 26.11 \\
\hline X-Light & 62.16 & 26.33 \\
\hline XX-Light & 64.14 & 26.64 \\
\hline
\end{tabular}

Table 5.13: Input Sub-Module for the Mean Reversion Price Forecasting Model

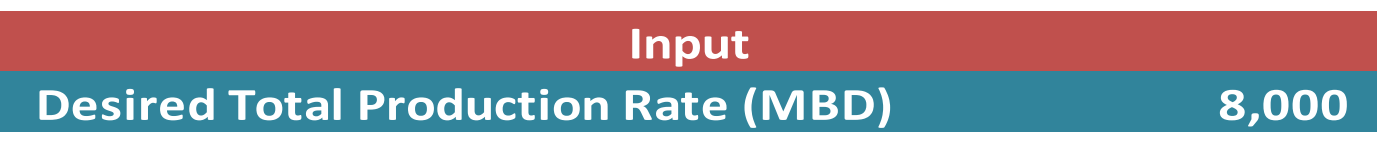

\begin{tabular}{|c|c|c|}
\hline \multicolumn{2}{|c|}{ Price Forecasting Parameters } & \\
\hline Heavy Crude & & \\
\hline$\eta($ Reversion Speed) & 1.50 & \\
\hline $\mathrm{P}^{*}$ (Long-Run Mean Price) & 56.23 & \\
\hline $\operatorname{Ln}\left(P^{*}\right)$ & 4.03 & \\
\hline$\sigma$ (Volatility) & 0.59 & \\
\hline Pmin(Heavy) & 40.00 & \\
\hline Crude & Mean Price $(\$)$ & Initial S.D. \\
\hline Heavy & 56.23 & 26.06 \\
\hline Medium & 57.99 & 26.08 \\
\hline Light & 60.36 & 26.11 \\
\hline X-Light & 62.16 & 26.33 \\
\hline XX-Light & 64.14 & 26.64 \\
\hline
\end{tabular}

Table 5.14: Input Sub-Module for the Mean Reversion with Jumps Price Forecasting Model 


\section{$\begin{array}{cc}\text { Input } & \\ \text { Desired Total Production Rate (MBD) } & \mathbf{8 , 0 0 0}\end{array}$}

\begin{tabular}{|c|c|}
\hline \multicolumn{2}{|c|}{ Price Forecasting $\mathrm{P}_{\mathrm{i}}$} \\
\hline \multicolumn{2}{|c|}{ Heavy Crude Parameters (Weekly) } \\
\hline n (Reversion Speed) & 0.01 \\
\hline P* (Long-Run Mean Price) & 56.23 \\
\hline $\ln \left(P^{*}\right)$ & 4.03 \\
\hline$\sigma$ (Volatility) & 0.05 \\
\hline$P_{\min }($ Heavy) & 40.00 \\
\hline$\Delta t$, (Years) & 0.02 \\
\hline Time Horizon, (Years) & 10.00 \\
\hline \multicolumn{2}{|c|}{ Initial Crude Prices } \\
\hline$P_{\text {Heavy }}(t=0)$ & 56.23 \\
\hline$P_{\text {Medium }}(t=0)$ & 57.99 \\
\hline$P_{\text {Light }}(t=0)$ & 60.36 \\
\hline$P_{X \text {-Light }}(t=0)$ & 62.16 \\
\hline$P_{X X-\text { Light }}(t=0)$ & 64.14 \\
\hline
\end{tabular}

\begin{tabular}{|lcl|}
\hline & Symbol & \multicolumn{2}{c|}{ Jump Parameters } \\
& Value & \multicolumn{1}{c|}{ Remarks } \\
\hline$\lambda$ & 0.13 & Jump Frequency \\
$\lambda^{*} \Delta t$ & 0.002 & Jump Probability \\
$\phi_{\text {Up }}$ & 0.69 & Jump Size Up \\
$\phi_{\text {Down }}$ & -0.69 & Jump Size Down \\
$\mathrm{E}\left[\phi^{2}\right]$ & 0.52 & Expected Value of Squared Jump Size \\
\hline
\end{tabular}

\subsection{Objective Function Sub-Module}

This sub-module is the first step in formulating the optimization model. The presence of the exponent term in the NPV equation orients the model toward nonlinearity. As a result, a non-linear solver was assigned to solve the optimization problem in the deterministic price forecasting approach. On the other hand, the Risk Solver Platform is capable of transforming a Monte Carlo simulation model into a deterministic 
equivalent and solving the optimization problem linearly. In the transformation process, the model is converted to a one with a huge number of variables to account for the uncertainty. Transforming the simulation model into a deterministic one and solving it linearly is a significant improvement of the model that reduces the computational time significantly. Any optimization problem has to answer three questions:

- What number to maximize/minimize (objective function)?

- What variable(s) to modify in order to achieve the objective function (decision variables)?

- What are the restrictions imposed on the decision variables (constraints)?

The objective function is the yardstick to measure the performance of a project or procedure. The most common yardstick for oil and gas projects is the net present value (NPV). The objective function was set to be the sum of all the field NPVs in the deterministic forecasting approach and the expected value of the total NPVs distribution in the probabilistic approach.

\subsection{Decision Variables Sub-Module}

Decision variables are the final output of the optimization model and are the parameters that management has control over. In other words, these variables lead to the answer of the study question presented in the introduction. The answer to the optimization problem is the set of decision variables that maximizes the total NPV (objective function). Table 15 shows a sample of optimum decision variables.

Table 5.15: Field Production Rate Allocation that Maximizes the Objective Function for Different Price Forecasting Models 


\begin{tabular}{|c|c|c|c|c|c|c|c|}
\hline Crude Grade & Field & Flat Price Model & $\begin{array}{c}\text { Floating Price } \\
\text { Model } \\
\end{array}$ & ILD Model & GBM Model & MRM Model & MRJM Model \\
\hline \multirow{3}{*}{ 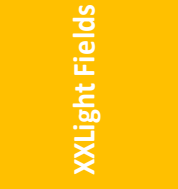 } & 1 & 153 & 153 & 153 & 153 & 153 & 153 \\
\hline & 2 & 329 & 329 & 329 & 329 & 329 & 329 \\
\hline & 3 & 205 & 205 & 205 & 205 & 205 & 205 \\
\hline \multirow{4}{*}{$\begin{array}{l}\frac{n}{0} \\
\frac{10}{14} \\
\frac{t}{00} \\
\frac{0}{x}\end{array}$} & 4 & 822 & 822 & 822 & 822 & 822 & 822 \\
\hline & 5 & 548 & 548 & 548 & 548 & 548 & 548 \\
\hline & 6 & 274 & 274 & 274 & 274 & 274 & 274 \\
\hline & 7 & 377 & 377 & 377 & 377 & 377 & 377 \\
\hline \multirow{8}{*}{ 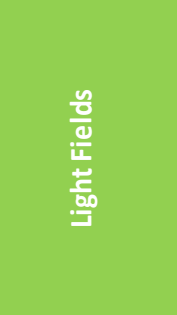 } & 8 & 33 & 110 & 33 & 110 & 33 & 33 \\
\hline & 9 & 247 & 247 & 247 & 247 & 247 & 247 \\
\hline & 10 & 342 & 342 & 342 & 342 & 342 & 342 \\
\hline & 11 & 548 & 548 & 548 & 548 & 548 & 548 \\
\hline & 12 & 822 & 822 & 822 & 822 & 822 & 822 \\
\hline & 13 & 438 & 438 & 438 & 438 & 438 & 438 \\
\hline & 14 & 438 & 438 & 438 & 438 & 438 & 438 \\
\hline & 15 & 411 & 411 & 411 & 411 & 411 & 411 \\
\hline \multirow{4}{*}{ 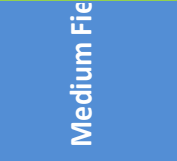 } & 16 & 123 & 123 & 123 & 123 & 123 & 123 \\
\hline & 17 & 308 & 308 & 308 & 308 & 308 & 308 \\
\hline & 18 & 247 & 247 & 247 & 247 & 247 & 247 \\
\hline & 19 & 545 & 468 & 545 & 468 & 545 & 545 \\
\hline \multirow{4}{*}{ 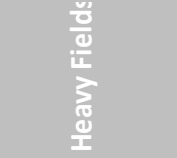 } & 20 & 205 & 205 & 205 & 205 & 205 & 205 \\
\hline & 21 & 222 & 222 & 222 & 222 & 222 & 222 \\
\hline & 22 & 197 & 197 & 197 & 197 & 197 & 197 \\
\hline & 23 & 164 & 164 & 164 & 164 & 164 & 164 \\
\hline
\end{tabular}

\subsection{Constraints Sub-Module}

Constraints are restrictions imposed on an optimization problem; these restrictions determine the range of the feasible solution of the problem. As mentioned above, there are 3 constraints for the optimization model in the study. First, field production rate must be less than or equal to the field production potential. Second, field production rate must be greater than or equal to the facility minimum operating rate. Third, total field production rate must meet the company's total-rate target. Field production potential is the maximum allowable field production rate while meeting reservoir engineers' requirements (meeting the demand, sufficient oil sweeping, maintain reservoir pressure). Facility minimum operating rate is the minimum rate that a facility must process to ensure safe and economical operation. 


\section{CHAPTER 6: RESULTS AND DISCUSSION}

Allocating production volumes across a portfolio of producing assets is a complex optimization problem. Each producing asset possesses different technical attributes (e.g. crude type), facility constraints, and costs; In addition to these field-level specifications, there are corporate objectives and constraints (e.g. contract delivery requirements). Price modeling assumption(s) is one of the most uncertain impacting parameters on the optimum allocation decision. This uncertainty adds to the complexity of the problem. Models necessary to implement the integrated "production allocation" stochastic optimization models have been defined: workflow designs to define the scope of analysis, field level engineering-economic evaluation models to calculate NPV values, stochastic processes to generate different sophisticated price models, regression analysis to correlate prices of different crude grades, Monte Carlo simulations to incorporate uncertainty, and optimization model to find the optimum set of decision variables. These components defined the workflow to determine the optimum production allocation, to investigate the impact of different price model assumption(s) on the production allocation decisions, and mitigate price uncertainties. In this section, the optimum allocation decisions resulted from different price model assumption(s) are presented and discussed. A sensitivity analysis is carried out in this section to determine the impact of price model assumption(s) on the total NPV.

\subsection{The Deterministic Price Models}

The deterministic approach is widely implemented in price forecasting within the oil industry due to its practicality and simplicity. This approach neglects price uncertainties, which may affect critical decisions. Here, the results of implanting the two 
deterministic price models are presented and analyzed (The two price models are discussed in the Price Modeling chapter).

\subsubsection{The Flat Price MOdel}

The Flat Price model is the simplest price model and assumes a fixed value for each crude grade throughout the life of the project. For this study, these price values are calculated as shown in the Price Modeling chapter using annual historical data between 2004 and 2010 as suggested by Xu (2010); Table 6.1 and Figure 6.1 show the Flat Price model.

Table 6.1: $\quad$ Flat Price Values

\begin{tabular}{|lcc|}
\hline \multicolumn{1}{|c}{ Crude } & Mean Price (\$) & Remarks \\
Heavy & 56.23 & Calculated \\
Medium & 57.99 & Calculated \\
Light & 60.36 & Calculated \\
X-Light & 62.16 & Assumed \\
XX-Light & 64.14 & Assumed \\
\hline
\end{tabular}




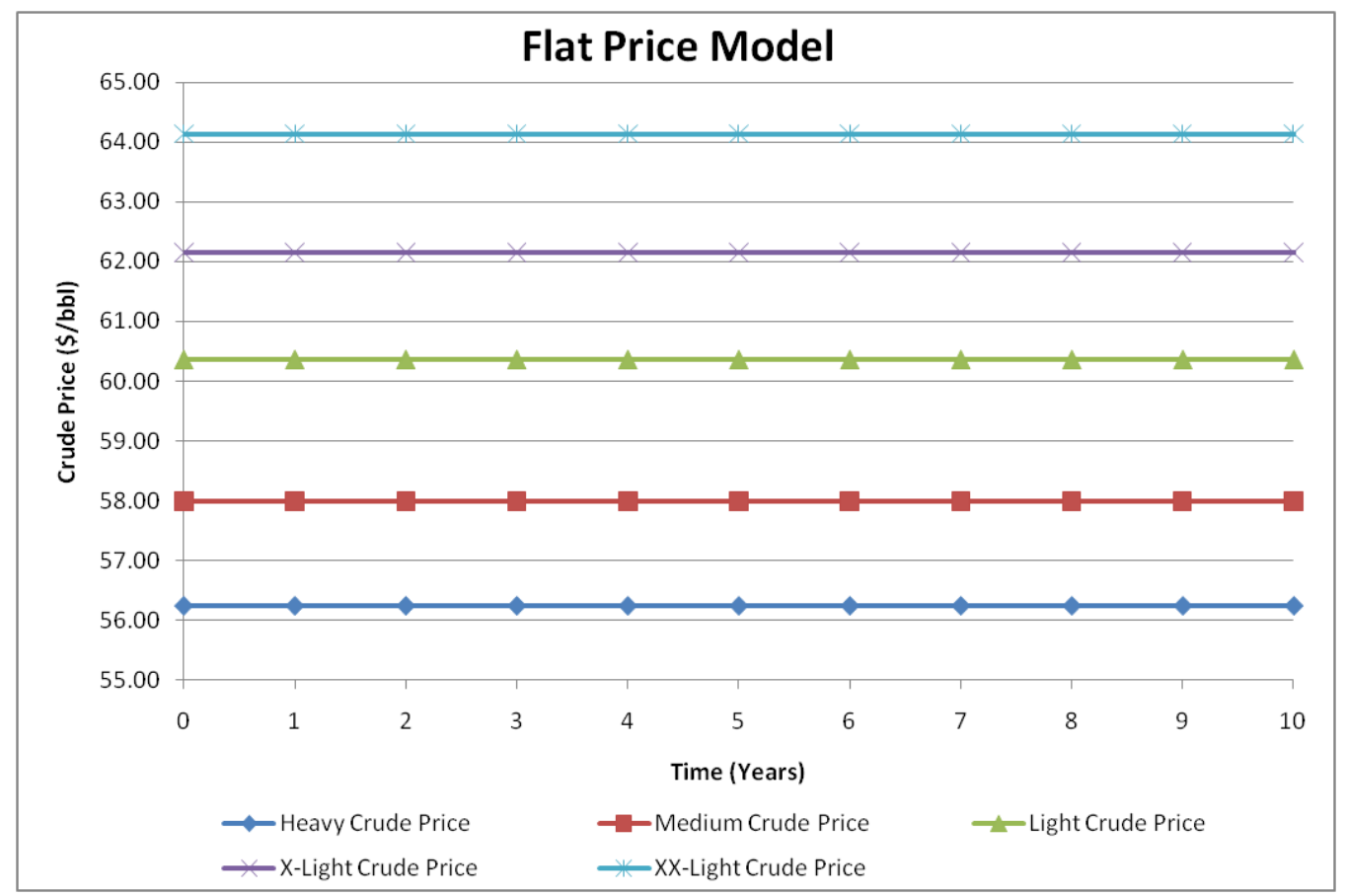

Figure 6.1: Flat Price Model

\subsubsection{The Optimum Decision of the Flat Price Model}

Table 6.2 shows the optimum production allocation decision when using the Flat Price model with total NPV of MM\$ 720,306. The optimizer places the XXLight, XLight, and Light crude fields at maximum potential rates except for Field 8, which is at the minimum operating rate. The Medium and Heavy crude fields are placed at the minimum operating rates except for Field 19, which is placed in the middle between its maximum and minimum rates. It is clear that the optimizer develops a workflow (workflow) to determine the optimum decisions. First, the optimizer fills all the fields with minimum operating rates to satisfy the lower bound constraint. Then, the algorithm places the XXLight and XLight fields at the maximum rates due to their high profitability. In the optimization process, the algorithm is deciding the production rate for each field to meet the corporate total target rate. After placing the XXLight and XLight 
fields at the maximum rates, the optimizer produces more from the higher value fields until the total target rate is met. The Light fields are placed at maximum rates except for Field 8; instead, the optimizer placed Field 19, a Medium crude field, in the middle between the maximum potential and minimum operating rates. This means that although Field 19 produces lower grade crude than Field 8, the production of Field 19 is more profitable. Lastly, the remaining Medium and Heavy fields are placed on minimum operating rates. Higher sale prices and lower production costs are among the reasons for the optimizer's optimum workflow. Lighter crude is less expensive to produce because of its lower viscosity and hence higher mobility within the reservoirs and lower reservoir depletion requirements. The Light grade is the dominant grade within the optimum production allocation as shown in Figure 6.3. Table 6.3 shows the optimum production allocation by crude grade, which reflects the available resources, which can be realized from the relatively small XXLight production rate. In other words, the XXLight crude fields are depleted due to the fact that the XXLight is the easy oil and has been produced over time. 
Table 6.2: Optimum Allocation Decision for Flat Price Model

\begin{tabular}{|c|c|c|c|}
\hline Crude Grade & Field & $\begin{array}{l}\text { Optimum } \\
\text { Production Rate } \\
\text { (MBD) }\end{array}$ & $\begin{array}{l}\text { Production } \\
\text { Rate Status }\end{array}$ \\
\hline$\frac{\frac{n}{0}}{\frac{0}{11}}$ & 1 & 153 & Upper Bound \\
\hline$\frac{\vec{t}}{6}$ & 2 & 329 & Upper Bound \\
\hline$\frac{x}{x}$ & 3 & 205 & Upper Bound \\
\hline \multirow{4}{*}{$\begin{array}{l}\frac{y}{0} \\
\frac{0}{4} \\
\frac{t}{60} \\
\frac{0}{x}\end{array}$} & 4 & 822 & Upper Bound \\
\hline & 5 & 548 & Upper Bound \\
\hline & 6 & 274 & Upper Bound \\
\hline & 7 & 377 & Upper Bound \\
\hline \multirow{8}{*}{ 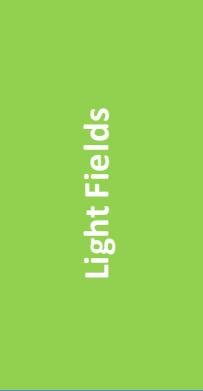 } & 8 & 33 & Lower_Bound \\
\hline & 9 & 247 & Upper Bound \\
\hline & 10 & 342 & Upper Bound \\
\hline & 11 & 548 & Upper Bound \\
\hline & 12 & 822 & Upper Bound \\
\hline & 13 & 438 & Upper Bound \\
\hline & 14 & 438 & Upper Bound \\
\hline & 15 & 411 & Upper Bound \\
\hline \multirow{4}{*}{ 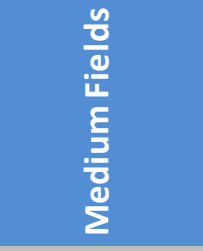 } & 16 & 123 & Lower_Bound \\
\hline & 17 & 308 & Lower_Bound \\
\hline & 18 & 247 & Lower_Bound \\
\hline & 19 & 545 & \\
\hline \multirow{4}{*}{ 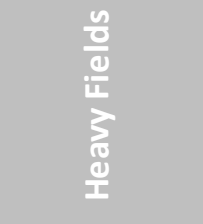 } & 20 & 205 & Lower_Bound \\
\hline & 21 & 222 & Lower_Bound \\
\hline & 22 & 197 & Lower_Bound \\
\hline & 23 & 164 & Lower_Bound \\
\hline
\end{tabular}


Table 6.3: $\quad$ Optimum Allocation Decision by Crude Grades for Flat Price Model

\begin{tabular}{|lc|}
\hline Crude Type & $\begin{array}{c}\text { Optimal Production } \\
\text { Rate (MBD) }\end{array}$ \\
$X X L$ & 688 \\
$X I$ & 2,021 \\
$A L$ & 3,279 \\
AM & 1,223 \\
AH & 789 \\
sum & 8,000 \\
\hline
\end{tabular}

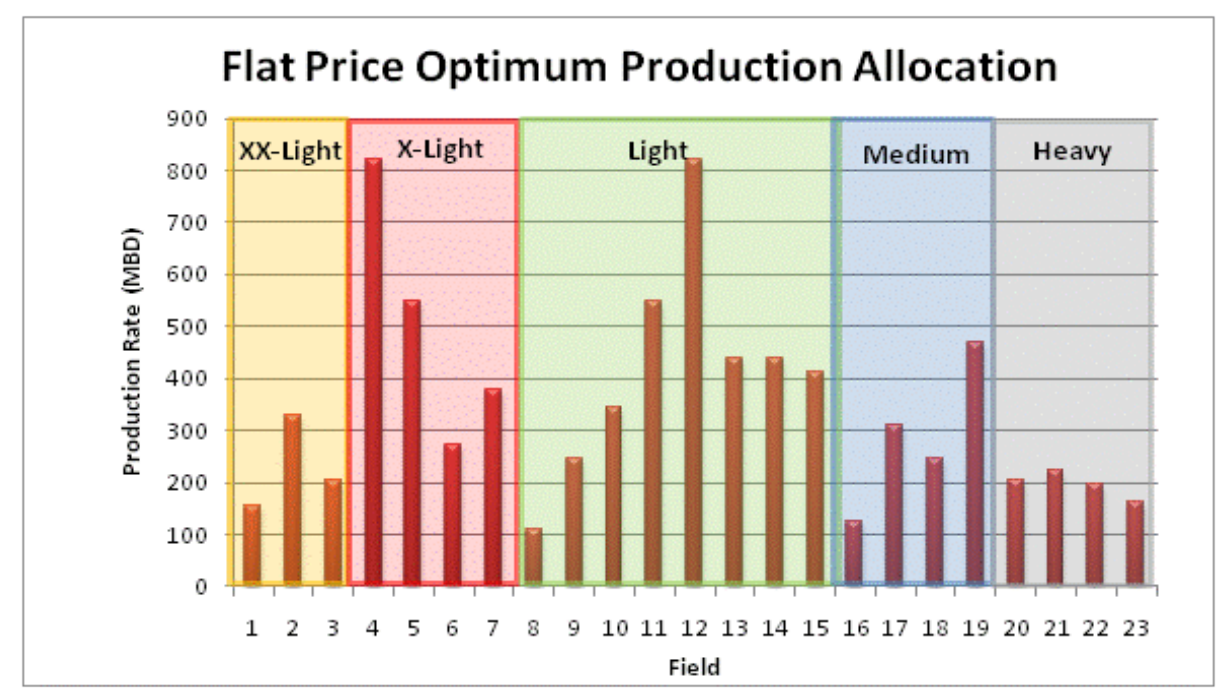

Figure 6.2: Optimum Production Allocation Decision for the Flat Price Model

\subsubsection{The Sensitivity Analysis of the Flat Price Model}

This section discusses the sensitivity of the total NPV to several price modeling parameters and how these parameters affect the optimum decisions. Figure 6.3 is the tornado chart for the total NPV in which the parameters are varied by $\pm 10 \%$. In other words, the chart shows how the total NPV is impacted by a $10 \%$ change in each parameter individually. As shown in the figure, Crude oil prices significantly impact the total NPV. Table 6.4 shows how the total NPV changes by a $\$ 1$ increase in the price of each crude; the impact shows how a crude grade is involved in the optimum allocation decision. The values from Table 6.4 are the slope of the lines in Figure 6.4; the steeper 
the line, the more significant the impact on the total NPV. These sensitivities reveal the importance of the price modeling assumptions on the total NPV calculated for the projects regardless of their impact on the optimum allocation decisions.

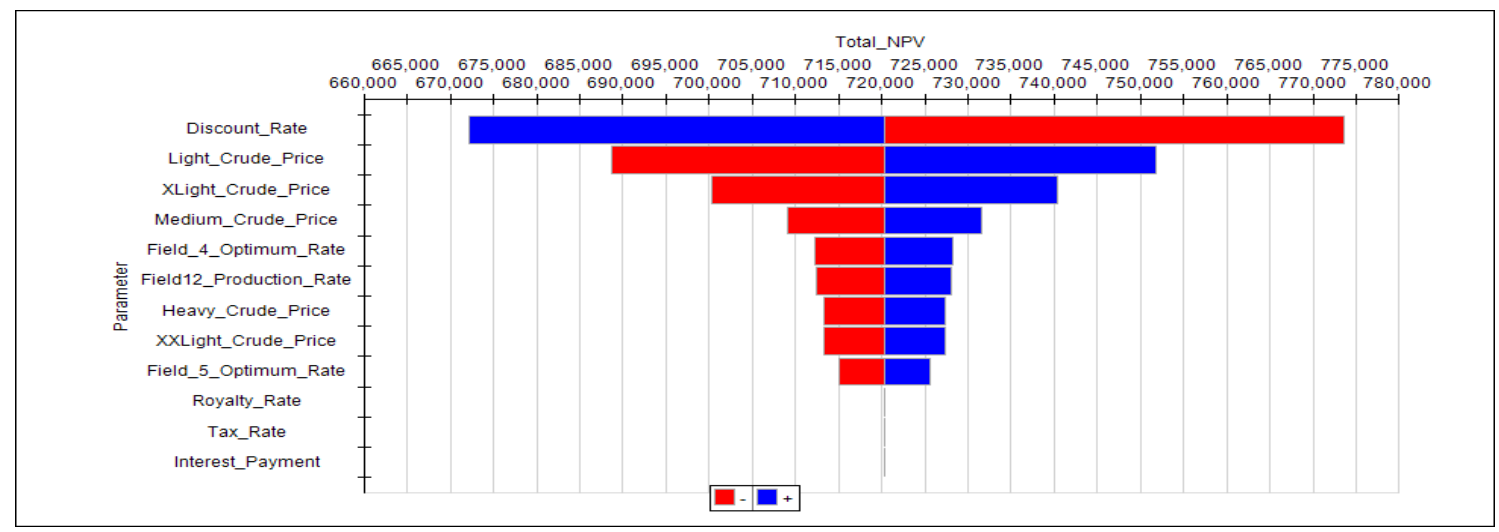

Figure 6.3: Total NPV Tornado Chart by Varying Parameters $\pm 10 \%$

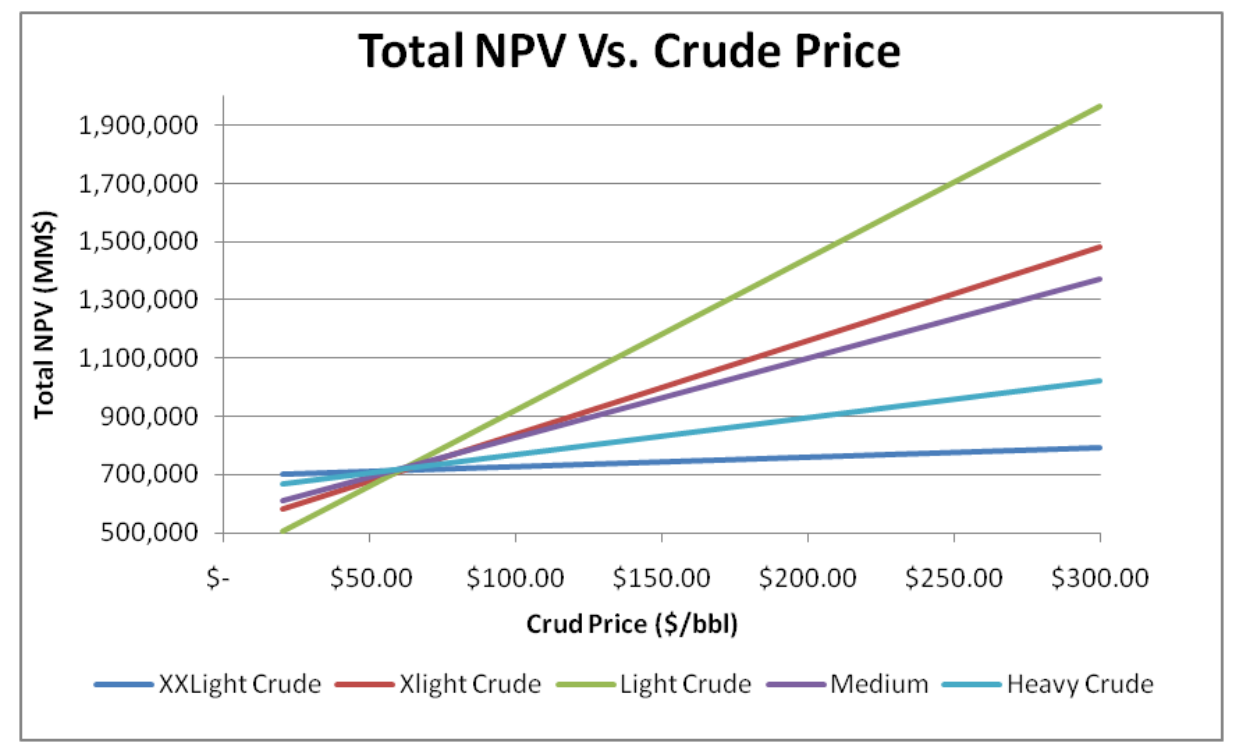

Figure 6.4: Price Impact on Total NPV within each Crude Grade 
Table 6.4: Total NPV Change per \$1 Increase in Prices

\begin{tabular}{|lc|}
\hline Crude Grade & $\begin{array}{c}\text { Total NPV Change per } \\
\text { \$1 Increase in Price } \\
\text { (MM\$) }\end{array}$ \\
XXLight & 329 \\
Xlighti & 3219 \\
Light & 5224 \\
Medium & 2715 \\
Heavy & 1257 \\
\hline
\end{tabular}

Table 6.5 shows the optimization sensitivity analysis of the XXLight crude prices, which is conducted by varying the price of the XXLight crude between $\$ 20$ and $\$ 300$ and observing the set of optimum allocation decisions. By varying the XXLight prices, deviations from the base case optimum decision can be observed in the XXLight fields and three of the Medium crude fields: Fields 17, 18 and 19. When the XXLight crude price is equal to or lower than $\$ 57.25$, the optimizer drops the XXLight fields to the minimum operating rates. This drop in total production rate is compensated by three of the Medium crude fields; the most profitable Medium crude field (Field 19) is placed at the maximum potential and the remaining $205 \mathrm{MBD}$ are compensated by Fields 17 and 18. When the XXLight price reaches $\$ 58.35$, the optimum allocation decision switches back to the base case. 
Table 6.5: The Optimization Sensitivity Analysis of the XXLight Crude Price

\begin{tabular}{|lcccccccc|}
\hline & \multicolumn{7}{c|}{ Production Rate } \\
\hline XXLight_Crude_Price & & Field 1 & Field 2 & Field 3 & Field 16 & Field 17 & Field 18 & Field 19 \\
\hline$\$$ & 20.00 & 46 & 99 & 62 & 123 & 411 & 349 & 822 \\
\hline$\$$ & 34.74 & 46 & 99 & 62 & 123 & 411 & 349 & 822 \\
\hline$\$$ & 57.25 & 46 & 99 & 62 & 123 & 411 & 349 & 822 \\
\hline$\$$ & $\mathbf{5 8 . 3 5}$ & 153 & 329 & 205 & 123 & 308 & 247 & 545 \\
\hline$\$$ & 78.95 & 153 & 329 & 205 & 123 & 308 & 247 & 545 \\
\hline$\$$ & 93.68 & 153 & 329 & 205 & 123 & 308 & 247 & 545 \\
\hline$\$$ & 108.42 & 153 & 329 & 205 & 123 & 308 & 247 & 545 \\
\hline$\$$ & 123.16 & 153 & 329 & 205 & 123 & 308 & 247 & 545 \\
\hline$\$$ & 137.89 & 153 & 329 & 205 & 123 & 308 & 247 & 545 \\
\hline$\$$ & 152.63 & 153 & 329 & 205 & 123 & 308 & 247 & 545 \\
\hline$\$$ & 167.37 & 153 & 329 & 205 & 123 & 308 & 247 & 545 \\
\hline$\$$ & 182.11 & 153 & 329 & 205 & 123 & 308 & 247 & 545 \\
\hline$\$$ & 196.84 & 153 & 329 & 205 & 123 & 308 & 247 & 545 \\
\hline$\$$ & 211.58 & 153 & 329 & 205 & 123 & 308 & 247 & 545 \\
\hline$\$$ & 226.32 & 153 & 329 & 205 & 123 & 308 & 247 & 545 \\
\hline$\$$ & 241.05 & 153 & 329 & 205 & 123 & 308 & 247 & 545 \\
\hline$\$$ & 255.79 & 153 & 329 & 205 & 123 & 308 & 247 & 545 \\
\hline$\$$ & 270.53 & 153 & 329 & 205 & 123 & 308 & 247 & 545 \\
\hline$\$$ & 285.26 & 153 & 329 & 205 & 123 & 308 & 247 & 545 \\
\hline$\$$ & 300.00 & 153 & 329 & 205 & 123 & 308 & 247 & 545 \\
\hline
\end{tabular}

Table 6.6 shows the optimization sensitivity analysis of the XLight crude prices, which is conducted by varying the price of the XLight crude between $\$ 20$ and $\$ 300$ and observing the set of optimum allocation decisions. By varying the XLight crude prices, deviations from the base case optimum decision can be observed in the XLight crude fields and three of the Medium crude fields: Fields 17, 18 and 19. When the XLight crude price is equal to or lower than $\$ 57.24$, the optimizer drops the XLight crude fields to the minimum operating rates. This drop in total production rate is compensated by three of the Medium crude fields; the most profitable Medium crude fields (Field 17 and 19) are placed at the maximum potential and the remaining 418 MBD are compensated by Field 18. When the XLight price reaches $\$ 58.35$, the optimum allocation decision switches back to the base case, which produces the XLight crude fields at maximum potential. 
Table 6.6: XLight Crude Price Optimization Sensitivity

\begin{tabular}{|lcccccccccc|}
\hline & & \multicolumn{7}{c|}{ Production Rate } & & \\
\hline XLight_Crude_Price & & Field 4 & Field 5 & Field 6 & Field 7 & Field 16 & Field 17 & Field 18 & Field 19 \\
\hline$\$$ & 20.00 & 247 & 164 & 82 & 113 & 123 & 1027 & 665 & 822 \\
\hline$\$$ & 34.74 & 247 & 164 & 82 & 113 & 123 & 1027 & 665 & 822 \\
\hline$\$$ & 57.24 & 247 & 164 & 82 & 113 & 123 & 1027 & 665 & 822 \\
\hline$\$$ & 57.94 & 822 & 548 & 274 & 377 & 123 & 308 & 247 & 545 \\
\hline$\$$ & 78.95 & 822 & 548 & 274 & 377 & 123 & 308 & 247 & 545 \\
\hline$\$$ & 93.68 & 822 & 548 & 274 & 377 & 123 & 308 & 247 & 545 \\
\hline$\$$ & 108.42 & 822 & 548 & 274 & 377 & 123 & 308 & 247 & 545 \\
\hline$\$$ & 123.16 & 822 & 548 & 274 & 377 & 123 & 308 & 247 & 545 \\
\hline$\$$ & 137.89 & 822 & 548 & 274 & 377 & 123 & 308 & 247 & 545 \\
\hline$\$$ & 152.63 & 822 & 548 & 274 & 377 & 123 & 308 & 247 & 545 \\
\hline$\$$ & 167.37 & 822 & 548 & 274 & 377 & 123 & 308 & 247 & 545 \\
\hline$\$$ & 182.11 & 822 & 548 & 274 & 377 & 123 & 308 & 247 & 545 \\
\hline$\$$ & 196.84 & 822 & 548 & 274 & 377 & 123 & 308 & 247 & 545 \\
\hline$\$$ & 211.58 & 822 & 548 & 274 & 377 & 123 & 308 & 247 & 545 \\
\hline$\$$ & 226.32 & 822 & 548 & 274 & 377 & 123 & 308 & 247 & 545 \\
\hline$\$$ & 241.05 & 822 & 548 & 274 & 377 & 123 & 308 & 247 & 545 \\
\hline$\$$ & 255.79 & 822 & 548 & 274 & 377 & 123 & 308 & 247 & 545 \\
\hline$\$$ & 270.53 & 822 & 548 & 274 & 377 & 123 & 308 & 247 & 545 \\
\hline$\$$ & 285.26 & 822 & 548 & 274 & 377 & 123 & 308 & 247 & 545 \\
\hline$\$$ & 300.00 & 822 & 548 & 274 & 377 & 123 & 308 & 247 & 545 \\
\hline & & & & & & & & &
\end{tabular}

Table 6.7 shows the optimization sensitivity analysis of the Light crude prices, which is conducted by varying the price of the Light crude between $\$ 40$ and $\$ 70$ and observing the set of optimum allocation decisions. By varying the Light crude prices, deviations from the base case optimum decision can be observed in the Light and Medium crude fields and Field 20, which is the most profitable field among the Heavy ones. When the Light crude price is equal to or lower than $\$ 54.48$, the optimizer drops the Light fields to the minimum operating rates. This huge drop in total production rate is compensated by the Medium crude fields and Field 20, which is Heavy crude. The Medium fields are placed at maximum potential; the remaining 414 MBD are compensated by the Heavy crude fields. Note that Field 20 is the most profitable field among the Heavy crude fields, but it is less profitable than all the Medium crude fields. At a Light price of $\$ 55.52$, the optimizer prefers Field 12 (Light crude) over the Field 20 
(Heavy crude), so the $414 \mathrm{MBD}$ is switched to Field 12. At a Light crude price of $\$ 57.59$, Fields 11, 12 and 14 (Light crude fields) are back to maximum potential rates; this additional rate is reduced from Fields 16, 17 and 18. At \$58.62 for the Light crude, production rates are switched from the Medium crude fields (17, 18 and 19) to the Light ones $(9,10,13$ and 15). Field 8, which is the least profitable Light crude field, becomes more profitable than Field 19 at $\$ 62.76$ for the Light crude; at that price, the optimum production allocation is back to the base case. The workflow used by the optimizer to determine the optimum allocation is obvious, and is discussed in the Discussion section.

Table 6.7: $\quad$ Light Crude Price Optimization Sensitivity

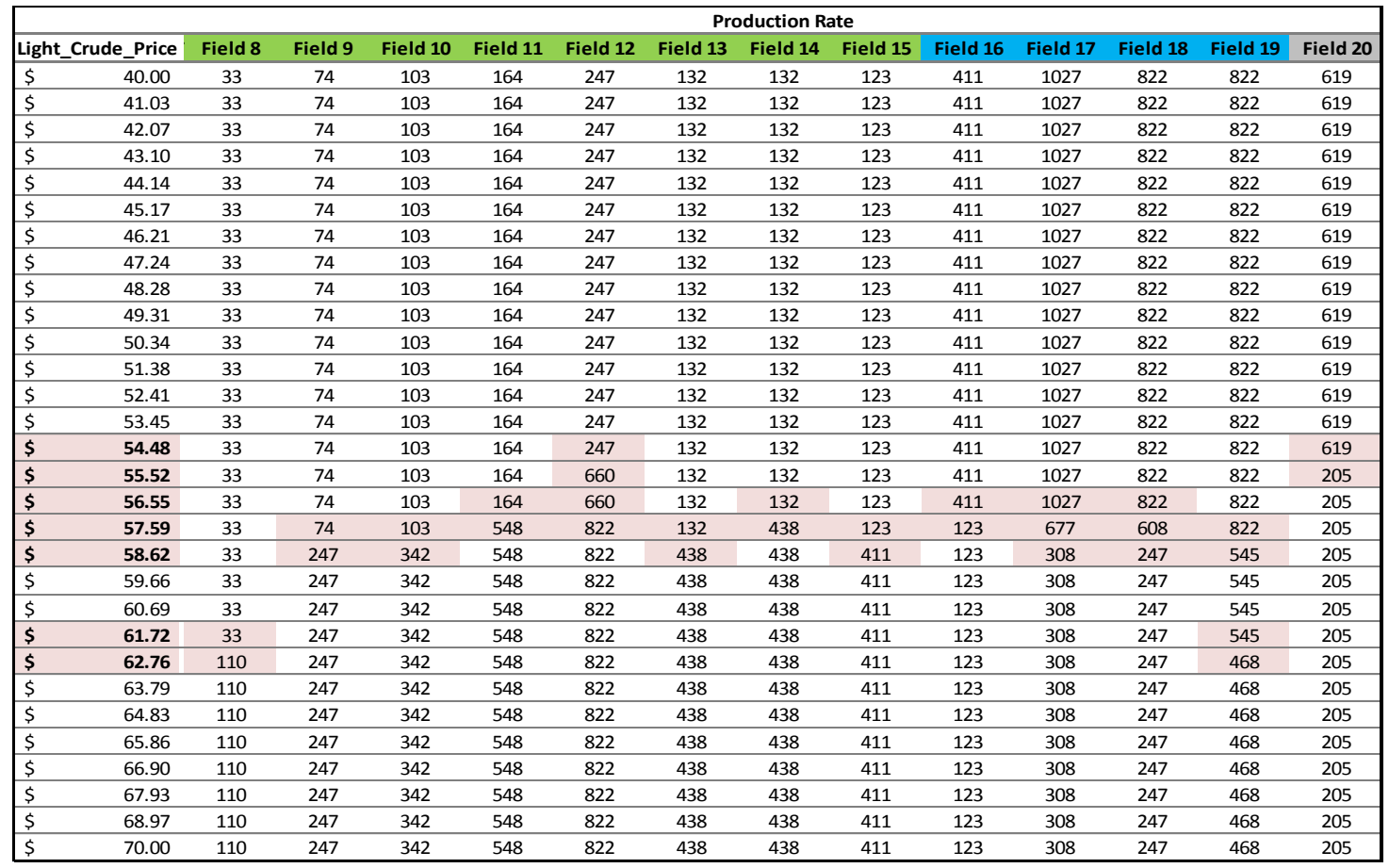

Table 6.8 shows the optimization sensitivity analysis of the Medium crude prices, which is conducted by varying the price between $\$ 40$ and $\$ 70$ and observing the set of optimum allocation decisions. The optimizer uses the same workflow as in the previous 
grades to achieve the optimum allocation decision. The critical Medium crude prices, where changes in the allocation decisions occur, are highlighted. Field 20 (Heavy) becomes more profitable than Field 19 (Medium crude) when the Medium crude price is lower than $\$ 56.55$. This shift in profitability results in a change in the optimum allocation decision as can be observed in Table 6.8. At $\$ 56.55$ for the Medium crude, the optimum allocation decision is the base case decision. When the Medium crude price reaches $\$ 61.72$, the Medium crude fields are more profitable than all the Light crude fields. As a result, the Medium crude fields dominate the production rate over the Light crude fields.

Table 6.8: $\quad$ Medium Crude Price Optimization Sensitivity

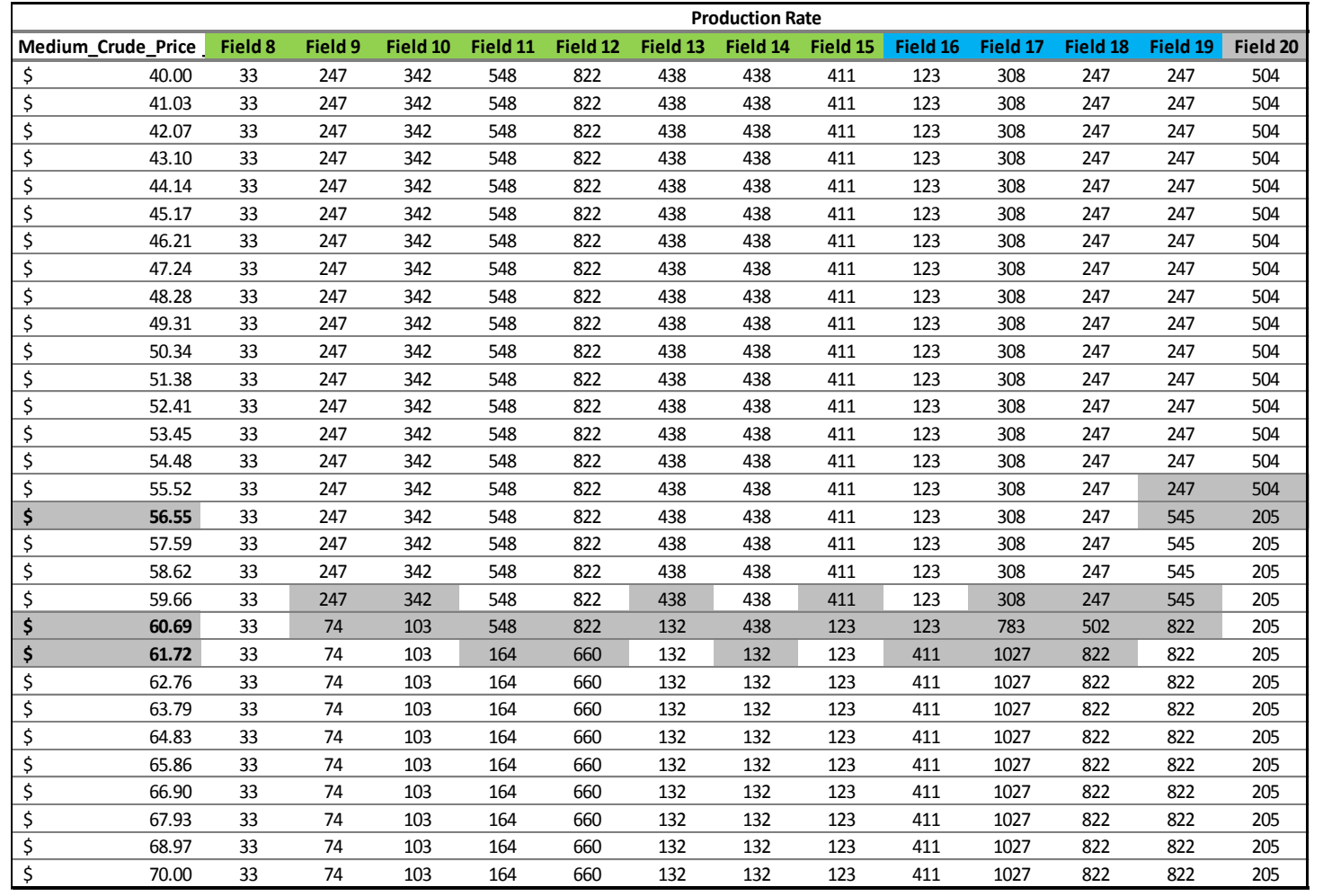


Table 6.9 shows the optimization sensitivity analysis of the Heavy crude prices, which is conducted by varying the price between $\$ 40$ and $\$ 70$ and observing the set of optimum allocation decisions. The optimizer uses the same workflow as in the previous grades to achieve the optimum allocation decision. The critical Heavy crude prices, where changes in the allocation decisions occur, are highlighted. As the Heavy crude price varies between $\$ 40.00$ and $\$ 57.59$, the optimum allocation decision is the base case. At a $\$ 58.62$ for the Heavy crude, Heavy crude fields take over the production of the Medium and Light crude fields as shown in Table 6.9. At a $\$ 61.72$ for the Heavy crude, all the Heavy crude fields are producing at maximum potential, which means that they are more profitable than the Medium and Light crude fields.

Table 6.9: Heavy Crude Price Optimization Sensitivity

\begin{tabular}{|c|c|c|c|c|c|c|c|c|c|c|c|c|c|c|}
\hline \multicolumn{2}{|c|}{ Heavy_Crude_Price } & \multicolumn{13}{|c|}{ Production Rate } \\
\hline \multicolumn{2}{|c|}{ Heavy_Crude_Price } & \multirow{2}{*}{$\begin{array}{r}\text { Field } 8 \\
33\end{array}$} & \multirow{2}{*}{$\begin{array}{r}\text { Field } 9 \\
247\end{array}$} & \multirow{2}{*}{$\begin{array}{r}\text { Field } 10 \\
342\end{array}$} & \multirow{2}{*}{$\begin{array}{r}\text { Field } 11 \\
548\end{array}$} & \multirow{2}{*}{$\begin{array}{r}\text { Field } 12 \\
822\end{array}$} & \multirow{2}{*}{$\begin{array}{r}\text { Field } 13 \\
438\end{array}$} & \multirow{2}{*}{$\begin{array}{r}\text { Field } 14 \\
438\end{array}$} & \multirow{2}{*}{$\begin{array}{r}\text { Field } 15 \\
411\end{array}$} & \multirow{2}{*}{$\begin{array}{r}\text { Field } 19 \\
545\end{array}$} & \multirow{2}{*}{$\begin{array}{r}\text { Field } 20 \\
205\end{array}$} & \multirow{2}{*}{$\begin{array}{r}\text { Field } 21 \\
222\end{array}$} & \multirow{2}{*}{$\begin{array}{r}\text { Field } 22 \\
197\end{array}$} & \multirow{2}{*}{$\begin{array}{r}\text { Field } 23 \\
164\end{array}$} \\
\hline$\$$ & 40.00 & & & & & & & & & & & & & \\
\hline$\$$ & 41.03 & 33 & 247 & 342 & 548 & 822 & 438 & 438 & 411 & 545 & 205 & 222 & 197 & 164 \\
\hline$\$$ & 43.10 & 33 & 247 & 342 & 548 & 822 & 438 & 438 & 411 & 545 & 205 & 222 & 197 & 164 \\
\hline$\$$ & 44.14 & 33 & 247 & 342 & 548 & 822 & 438 & 438 & 411 & 545 & 205 & 222 & 197 & 164 \\
\hline$\$$ & 45.17 & 33 & 247 & 342 & 548 & 822 & 438 & 438 & 411 & 545 & 205 & 222 & 197 & 164 \\
\hline$\$$ & 47.24 & 33 & 247 & 342 & 548 & 822 & 438 & 438 & 411 & 545 & 205 & 222 & 197 & 164 \\
\hline$\$$ & 48.28 & 33 & 247 & 342 & 548 & 822 & 438 & 438 & 411 & 545 & 205 & 222 & 197 & 164 \\
\hline$\$$ & 49.31 & 33 & 247 & 342 & 548 & 822 & 438 & 438 & 411 & 545 & 205 & 222 & 197 & 164 \\
\hline$\$$ & 50.34 & 33 & 247 & 342 & 548 & 822 & 438 & 438 & 411 & 545 & 205 & 222 & 197 & 164 \\
\hline$\$$ & 51.38 & 33 & 247 & 342 & 548 & 822 & 438 & 438 & 411 & 545 & 205 & 222 & 197 & 164 \\
\hline$\$$ & 52.41 & 33 & 247 & 342 & 548 & 822 & 438 & 438 & 411 & 545 & 205 & 222 & 197 & 164 \\
\hline$\$$ & 57.59 & 33 & 247 & 342 & 548 & 822 & 438 & 438 & 411 & 545 & 205 & 222 & 197 & 164 \\
\hline$\$$ & 58.62 & 33 & 247 & 342 & 548 & 822 & 438 & 438 & 411 & 247 & 504 & 222 & 197 & 164 \\
\hline$\$$ & 59.66 & 33 & 247 & 342 & 548 & 822 & 438 & 438 & 411 & 247 & 504 & 222 & 197 & 164 \\
\hline$\$$ & 60.69 & 33 & 247 & 103 & 548 & 822 & 438 & 438 & 123 & 247 & 685 & 568 & 197 & 164 \\
\hline$\$$ & 61.72 & 33 & 74 & 103 & 319 & 822 & 132 & 132 & 123 & 247 & 685 & 740 & 658 & 548 \\
\hline$\$$ & 62.76 & 33 & 74 & 103 & 319 & 822 & 132 & 132 & 123 & 247 & 685 & 740 & 658 & 548 \\
\hline$\$$ & 63.79 & 33 & 74 & 103 & 319 & 822 & 132 & 132 & 123 & 247 & 685 & 740 & 658 & 548 \\
\hline$\$$ & 64.83 & 33 & 74 & 103 & 319 & 822 & 132 & 132 & 123 & 247 & 685 & 740 & 658 & 548 \\
\hline$\$$ & 65.86 & 33 & 74 & 103 & 319 & 822 & 132 & 132 & 123 & 247 & 685 & 740 & 658 & 548 \\
\hline$\$$ & 66.90 & 33 & 74 & 103 & 319 & 822 & 132 & 132 & 123 & 247 & 685 & 740 & 658 & 548 \\
\hline$\$$ & 67.93 & 33 & 74 & 103 & 319 & 822 & 132 & 132 & 123 & 247 & 685 & 740 & 658 & 548 \\
\hline
\end{tabular}




\subsubsection{The Floating Price Model}

The Floating Price model is one of the commonly used crude oil price forecasting models in the oil industry. The model's popularity comes from the old belief that oil is an inelastic commodity. In economics, an inelastic commodity means that the commodity price does not respond to changes in the oil demand, so oil industry experts thought that crude oil prices should continue to increase regardless of the change in demand. An explanation for this theory is that there are limited and scarce crude oil resources.

This increasing price model requires an initial price and a rate of increase; Table 6.10 shows the initial prices and rate of price increases required for the model. As shown in the table, the parameters of three of the crudes are calculated from the historical data and the remaining ones are estimated from these calculated parameters as discussed in the chapter on Crude and Pricing. Figure 6.5 shows the Floating Price model.

Table 6.10: Floating Price Model Parameters

\begin{tabular}{|lccl|}
\hline \multicolumn{1}{c}{ Crude } & Mean Price (\$) & Growth Rate & Remarks \\
\cline { 2 - 4 } Heavy & 56.23 & 0.22 & Calculated \\
Medium & 57.99 & 0.23 & Calculated \\
Light & 60.36 & 0.25 & Calculated \\
X-Light & 62.16 & 0.27 & Assumed \\
XX-Light & 64.14 & 0.30 & Assumed \\
\hline
\end{tabular}




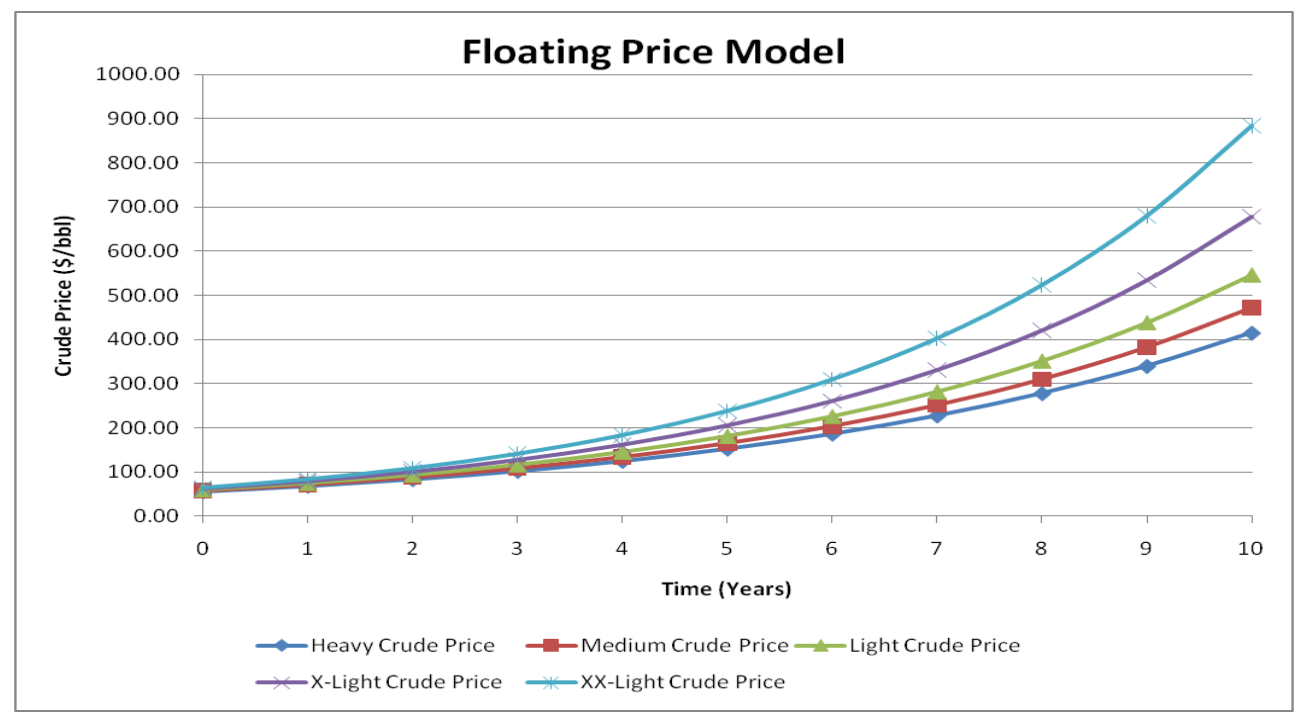

Figure 6.5: Floating Price Model

\subsubsection{The Optimum Decision of the Floating Price Model}

Table 6.11 shows the optimum production allocation decision when using the Floating Price model with total NPV of MM\$3,154,895. The table shows that the optimum decisions for the Floating and Flat price models are similar; the only difference is that the optimizer replaces 77 MBD from Field 19 (Medium crude) with Field 8 (Light crude) when using the Floating Price forecasting model. In other words, Field 8 is more valuable than Field 19 to the optimizer. Similar to the Flat Price case, it is clear that the optimizer develops a workflow (trend) to rank the fields and to determine the optimum decisions. First, the optimizer fills all the fields with minimum operating rates to satisfy the lower bound constraint. Then, the algorithm places the fields of the XXLight crude and XLight crude at maximum rates due to their high profitability. In the optimization process, the algorithm is deciding the production rate for each field to meet the corporate total target rate. After placing the fields of the XXLight crude and XLight crude at maximum rates, the optimizer produces more from the higher value fields until the corporate target is met. The Light crude fields are placed at maximum rates. Lastly, Field 
19 is assigned 468 MBD to meet the management requirement. Higher sale prices and lower production costs are among the reasons for the allocation workflow used by the optimizer. Lighter crude is less expensive to produce because of its lower viscosity and hence higher mobility within the reservoirs and lower reservoir depletion requirements. The Light grade is the dominant grade within the optimum production allocation as shown in Figure 6.6. Table 6.12 shows the optimum production allocation by crude grade, which reflects the available resources, which can be realized from the relatively small production rate of the XXLight crude. In other words, the XXLight crude fields are depleted due to the fact that the XXLight crude is the "easy oil" and has been produced over the last decades. 
Table 6.11: Optimum Allocation for Floating Price Model

\begin{tabular}{|c|c|c|c|c|c|}
\hline \multirow[b]{2}{*}{ Crude Grade } & \multicolumn{3}{|c|}{ "Floating Price" Optimum } & \multirow{2}{*}{$\begin{array}{c}\text { "Flat Price" Optimum } \\
\text { Production Rate }\end{array}$} & \multirow{2}{*}{$\begin{array}{c}\text { Difference } \\
\text { Between Current } \\
\text { and Flat Price Case }\end{array}$} \\
\hline & Field & $\begin{array}{l}\text { Production Rate } \\
\text { (MBD) }\end{array}$ & Production Rate Status & & \\
\hline \multirow{3}{*}{ 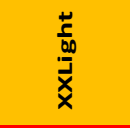 } & 1 & 153 & Upper Bound & 153 & 0 \\
\hline & 2 & 329 & Upper Bound & 329 & 0 \\
\hline & 3 & 205 & Upper Bound & 205 & 0 \\
\hline \multirow{4}{*}{ 妾 } & 4 & 822 & Upper Bound & 822 & 0 \\
\hline & 5 & 548 & Upper Bound & 548 & 0 \\
\hline & 6 & 274 & Upper Bound & 274 & 0 \\
\hline & 7 & 377 & Upper Bound & 377 & 0 \\
\hline \multirow{8}{*}{ 㠓 } & 8 & 110 & Upper Bound & 33 & 77 \\
\hline & 9 & 247 & Upper Bound & 247 & 0 \\
\hline & 10 & 342 & Upper Bound & 342 & 0 \\
\hline & 11 & 548 & Upper Bound & 548 & 0 \\
\hline & 12 & 822 & Upper Bound & 822 & 0 \\
\hline & 13 & 438 & Upper Bound & 438 & 0 \\
\hline & 14 & 438 & Upper Bound & 438 & 0 \\
\hline & 15 & 411 & Upper Bound & 411 & 0 \\
\hline \multirow{4}{*}{$\begin{array}{l}\xi \\
\frac{\xi}{3} \\
\frac{\partial}{0} \\
\sum \\
\Sigma\end{array}$} & 16 & 123 & Lower_Bound & 123 & 0 \\
\hline & 17 & 308 & Lower_Bound & 308 & 0 \\
\hline & 18 & 247 & Lower_Bound & 247 & 0 \\
\hline & 19 & 468 & & 545 & -77 \\
\hline \multirow{4}{*}{$\begin{array}{l}\sum_{0} \\
0 \\
I \\
I\end{array}$} & 20 & 205 & Lower_Bound & 205 & 0 \\
\hline & 21 & 222 & Lower_Bound & 222 & 0 \\
\hline & 22 & 197 & Lower_Bound & 197 & 0 \\
\hline & 23 & 164 & Lower_Bound & 164 & 0 \\
\hline
\end{tabular}

Table 6.12: Optimum Allocation by Crude Grade for Floating Price Model

Crude Grade Optimal Production Rate (MBD)
\begin{tabular}{|lc|} 
XXL & 688 \\
XI & 2,021 \\
AL & 3,356 \\
AM & 1,147 \\
AH & 789 \\
Total & 8,000 \\
\hline
\end{tabular}




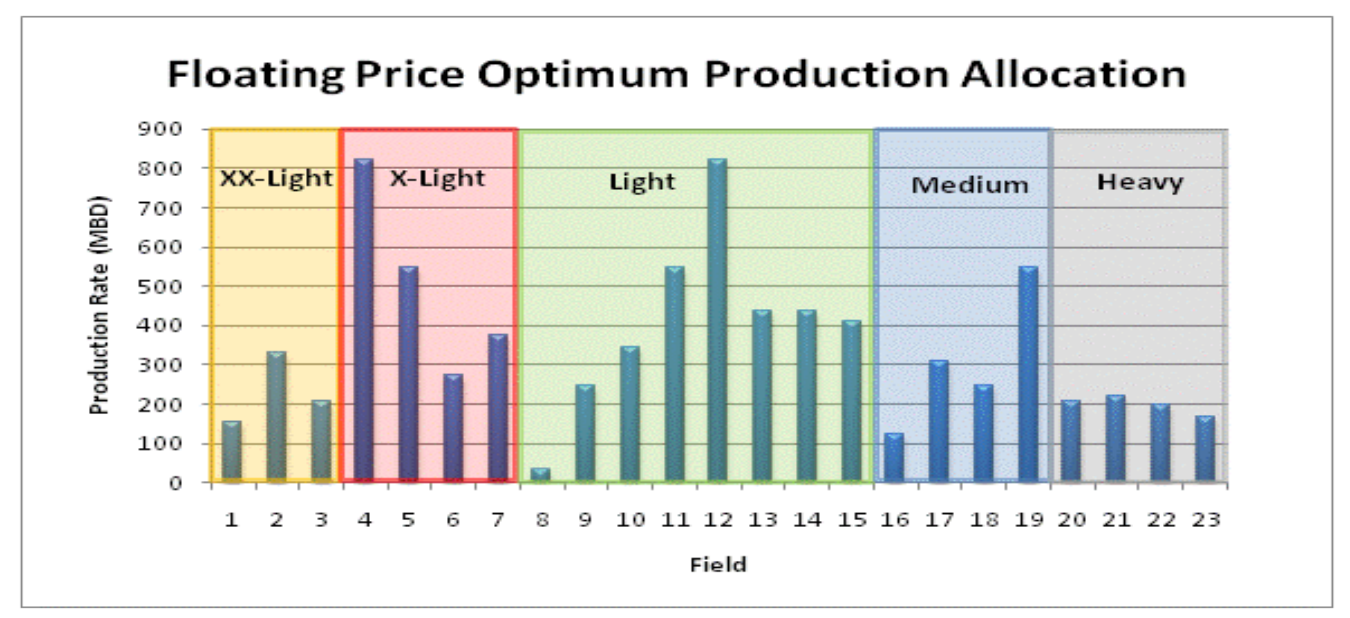

Figure 6.6: Optimum Production Allocation Decision for Floating Price model

\subsubsection{The Sensitivity Analysis of the Floating Price Model}

Here, we identify the most significant parameters for the total NPV calculations and show how these parameters affect the optimum allocation decision. Figure 6.7 is the tornado chart for the total NPV that is generated by varying the parameters by $\pm 10 \%$. Each parameter was analyzed individually to observe its affect on the total NPV. The tornado chart reveals the significance of the price modeling parameters on the value of the total NPV. Sensitivity analysis focuses on the rate of price increases because it is the most significant price modeling parameter for the total NPV. Figure 6.8 shows the total NPV responses to the changes in the rate of price increases for all crude grades. The figure implies that estimating the rate of price increases is critical for the Light and the XLight crudes. On the other hand, estimating the rate of price increases for the XXLight and the Heavy crudes is not as critical. Another characteristic of the figure is that the total NPV gain from a $1 \%$ increase in the rate of price increases is not constant since the curves are not linear. The additional NPV value resulted from a $1 \%$ increase in the rate of price increases is significantly larger at higher rates of increase. 


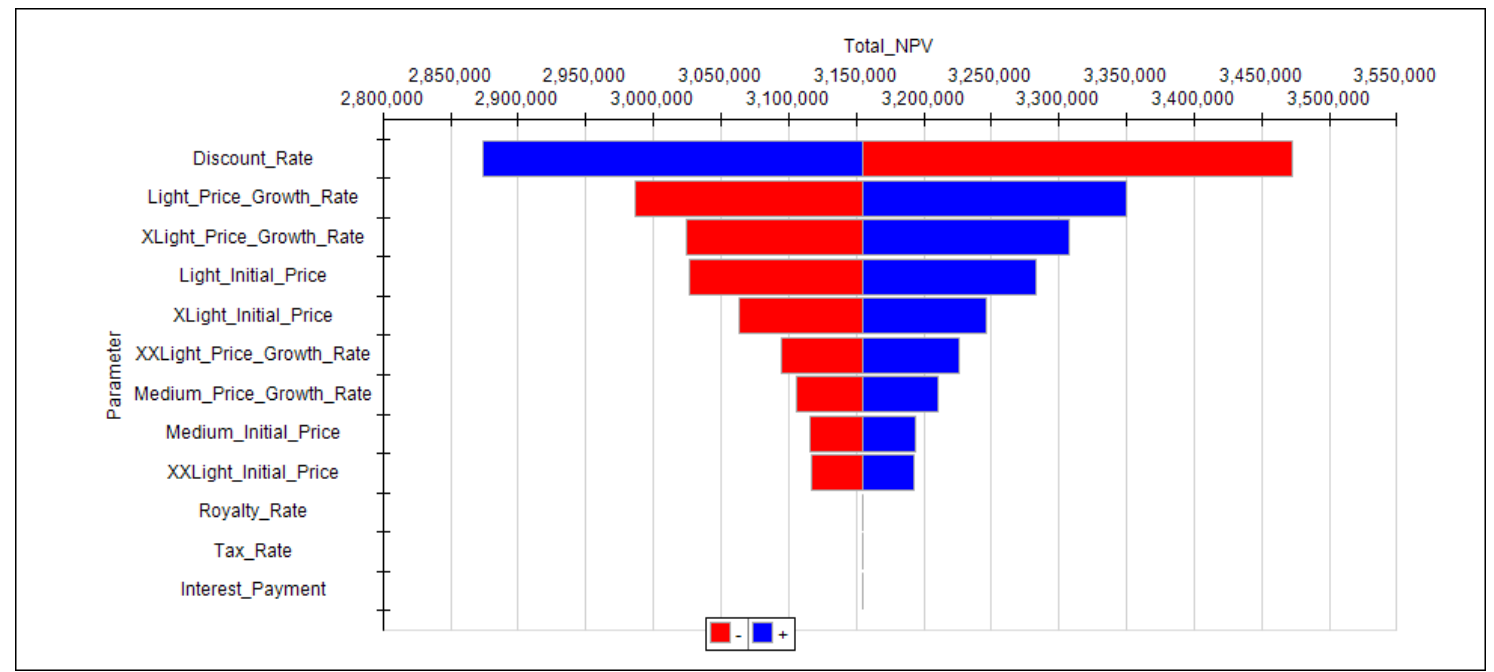

Figure 6.7: Total NPV Tornado Chart by Varying Parameters $\pm 10 \%$

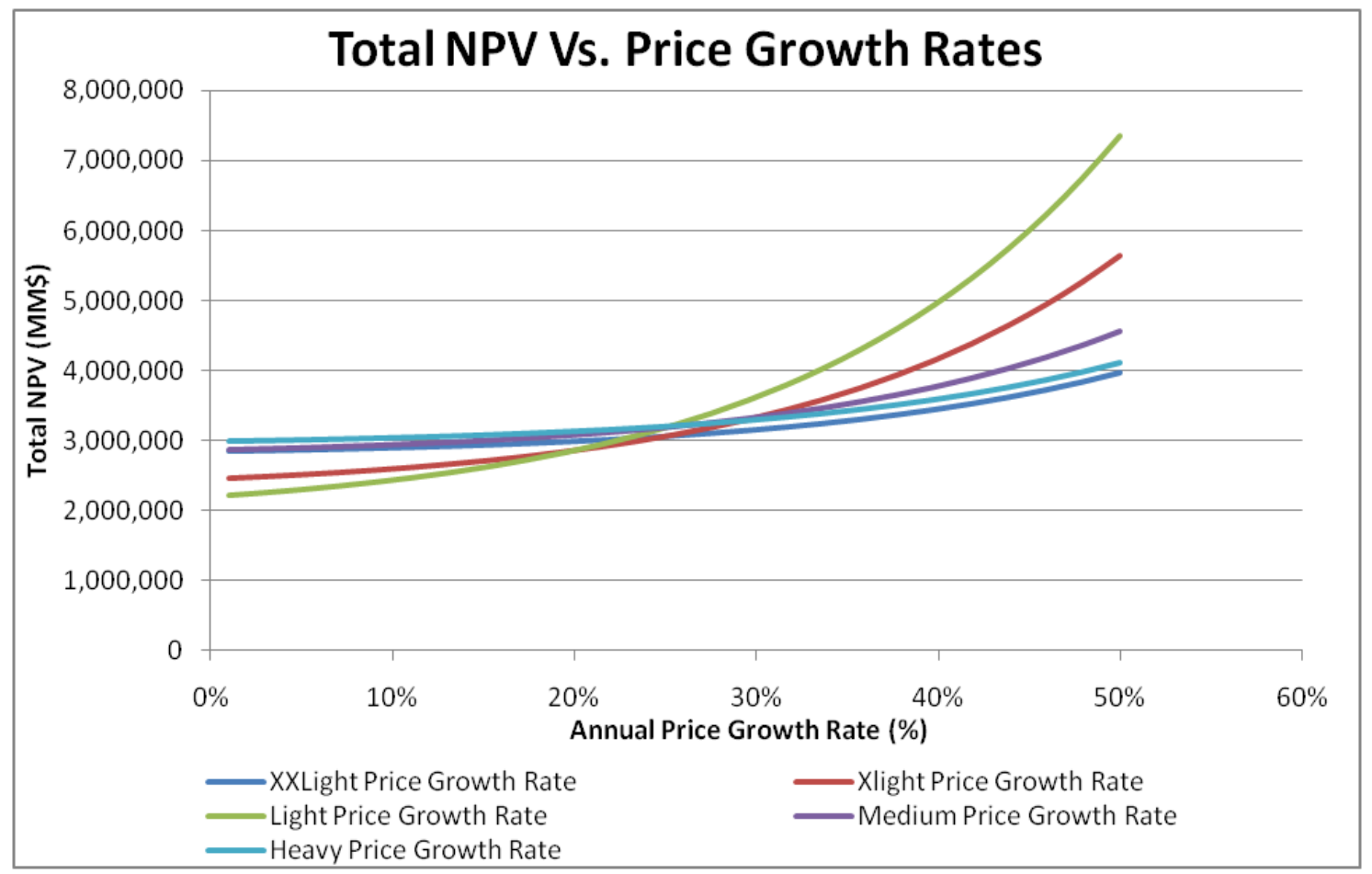

Figure 6.8: The Impact of Price Growth Rates on the Total NPV

Table 6.13 presents the sensitivity analysis of the rate of price increases for the XXLight crude. The analysis was conducted by varying the rate of price increases 
between $1 \%$ and $40 \%$, and observing the changes in the optimum allocation decision.

Starting with a $1 \%$ rate of increase, all the XXLight crude fields were placed on minimum operating rates; this production loss in the XXLight crude fields was compensated by the Medium crude fields (Fields 17 and 19). At 23\% growth rate, the optimum allocation decision returns to the base case.

Table 6.13: XXLight Price Growth Rate Optimization Sensitivity

\begin{tabular}{|c|c|c|c|c|c|c|c|c|}
\hline \multirow[b]{2}{*}{ XXLight_Price_Growth_Rate } & \multirow[b]{2}{*}{ Total_NPV } & \multicolumn{7}{|c|}{ Production Rate } \\
\hline & & Field 1 & Field 2 & Field 3 & Field 16 & Field 17 & Field 18 & Field 19 \\
\hline $1 \%$ & $2,960,754$ & 46 & 99 & 62 & 123 & 436 & 247 & 822 \\
\hline $2 \%$ & $2,962,435$ & 46 & 99 & 62 & 123 & 436 & 247 & 822 \\
\hline $4 \%$ & $2,964,254$ & 46 & 99 & 62 & 123 & 436 & 247 & 822 \\
\hline $5 \%$ & $2,966,220$ & 46 & 99 & 62 & 123 & 436 & 247 & 822 \\
\hline $6 \%$ & $2,968,347$ & 46 & 99 & 62 & 123 & 436 & 247 & 822 \\
\hline $8 \%$ & $2,970,648$ & 46 & 99 & 62 & 123 & 436 & 247 & 822 \\
\hline $9 \%$ & $2,973,138$ & 46 & 99 & 62 & 123 & 436 & 247 & 822 \\
\hline $10 \%$ & $2,975,833$ & 46 & 99 & 62 & 123 & 436 & 247 & 822 \\
\hline $12 \%$ & $2,978,749$ & 46 & 99 & 62 & 123 & 436 & 247 & 822 \\
\hline $13 \%$ & $2,981,906$ & 46 & 99 & 62 & 123 & 436 & 247 & 822 \\
\hline $14 \%$ & $2,985,323$ & 46 & 99 & 62 & 123 & 436 & 247 & 822 \\
\hline $16 \%$ & $2,989,021$ & 46 & 99 & 62 & 123 & 436 & 247 & 822 \\
\hline $17 \%$ & $2,993,024$ & 46 & 99 & 62 & 123 & 436 & 247 & 822 \\
\hline $18 \%$ & $2,997,356$ & 46 & 99 & 62 & 123 & 436 & 247 & 822 \\
\hline $20 \%$ & $3,002,044$ & 46 & 99 & 62 & 123 & 436 & 247 & 822 \\
\hline $21 \%$ & $3,007,118$ & 46 & 99 & 62 & 123 & 436 & 247 & 822 \\
\hline $23 \%$ & $3,022,159$ & 153 & 329 & 205 & 123 & 308 & 247 & 468 \\
\hline $24 \%$ & $3,041,957$ & 153 & 329 & 205 & 123 & 308 & 247 & 468 \\
\hline $25 \%$ & $3,063,373$ & 153 & 329 & 205 & 123 & 308 & 247 & 468 \\
\hline $27 \%$ & $3,086,536$ & 153 & 329 & 205 & 123 & 308 & 247 & 468 \\
\hline $28 \%$ & $3,111,583$ & 153 & 329 & 205 & 123 & 308 & 247 & 468 \\
\hline $29 \%$ & $3,138,663$ & 153 & 329 & 205 & 123 & 308 & 247 & 468 \\
\hline $31 \%$ & $3,167,934$ & 153 & 329 & 205 & 123 & 308 & 247 & 468 \\
\hline $32 \%$ & $3,199,566$ & 153 & 329 & 205 & 123 & 308 & 247 & 468 \\
\hline $33 \%$ & $3,233,742$ & 153 & 329 & 205 & 123 & 308 & 247 & 468 \\
\hline $35 \%$ & $3,270,657$ & 153 & 329 & 205 & 123 & 308 & 247 & 468 \\
\hline $36 \%$ & $3,310,521$ & 153 & 329 & 205 & 123 & 308 & 247 & 468 \\
\hline $37 \%$ & $3,353,556$ & 153 & 329 & 205 & 123 & 308 & 247 & 468 \\
\hline $39 \%$ & $3,400,003$ & 153 & 329 & 205 & 123 & 308 & 247 & 468 \\
\hline $40 \%$ & $3,450,117$ & 153 & 329 & 205 & 123 & 308 & 247 & 468 \\
\hline
\end{tabular}


The sensitivity analysis of the rate of increase in the price of the XLight is presented in Table 6.14 , and is conducted by varying the rate of increase between $1 \%$ and $40 \%$, and by observing the changes in the optimum allocation decision. Similar to the analysis of the rate of the XXLight price increases, all the XLight crude fields were placed at minimum operating rates when the rate of the XLight price increases is low. The production loss is compensated by all the Medium crude fields. At a 23\% increase in the price of the XLight crude, the optimum allocation decision is back to the base case. Note that the optimizer develops a workflow (procedure) to allocate the production rate, similar to the Flat Price model. The workflow shows that the XXLight crude fields are ranked first; followed by Xlight, Light, Medium, and lastly Heavy crude fields. In other words, the XXLight fields are placed on maximum potential before the other grade fields.

Table 6.14: XLight Price Growth Rate Optimization Sensitivity

\begin{tabular}{|c|c|c|c|c|c|c|c|c|c|}
\hline \multirow[b]{2}{*}{ XLight_Price_Growth Rate } & \multirow[b]{2}{*}{ Total NPV } & \multicolumn{8}{|c|}{ Production Rate } \\
\hline & & Field 4 & Field 5 & Field 6 & Field 7 & Field 16 & Field 17 & Field 18 & Field 19 \\
\hline $1 \%$ & $2,788,524$ & 247 & 164 & 82 & 113 & 411 & 958 & 370 & 822 \\
\hline $2 \%$ & $2,793,312$ & 247 & 164 & 82 & 113 & 411 & 958 & 370 & 822 \\
\hline $4 \%$ & $2,798,489$ & 247 & 164 & 82 & 113 & 411 & 958 & 370 & 822 \\
\hline $5 \%$ & $2,804,088$ & 247 & 164 & 82 & 113 & 411 & 958 & 370 & 822 \\
\hline $6 \%$ & $2,810,145$ & 247 & 164 & 82 & 113 & 411 & 958 & 370 & 822 \\
\hline $8 \%$ & $2,816,698$ & 247 & 164 & 82 & 113 & 411 & 958 & 370 & 822 \\
\hline $9 \%$ & $2,823,788$ & 247 & 164 & 82 & 113 & 411 & 958 & 370 & 822 \\
\hline $10 \%$ & $2,831,462$ & 247 & 164 & 82 & 113 & 411 & 958 & 370 & 822 \\
\hline $12 \%$ & $2,839,767$ & 247 & 164 & 82 & 113 & 411 & 958 & 370 & 822 \\
\hline $13 \%$ & $2,848,756$ & 247 & 164 & 82 & 113 & 411 & 958 & 370 & 822 \\
\hline $14 \%$ & $2,858,485$ & 247 & 164 & 82 & 113 & 411 & 958 & 370 & 822 \\
\hline $16 \%$ & $2,869,016$ & 247 & 164 & 82 & 113 & 411 & 958 & 370 & 822 \\
\hline $17 \%$ & $2,880,414$ & 247 & 164 & 82 & 113 & 411 & 958 & 370 & 822 \\
\hline $18 \%$ & $2,892,750$ & 247 & 164 & 82 & 113 & 411 & 958 & 370 & 822 \\
\hline $20 \%$ & $2,906,101$ & 247 & 164 & 82 & 113 & 411 & 958 & 370 & 822 \\
\hline $21 \%$ & $2,920,549$ & 247 & 164 & 82 & 113 & 411 & 958 & 370 & 822 \\
\hline $23 \%$ & $2,948,419$ & 822 & 548 & 274 & 377 & 123 & 308 & 247 & 468 \\
\hline $24 \%$ & $3,004,795$ & 822 & 548 & 274 & 377 & 123 & 308 & 247 & 468 \\
\hline $25 \%$ & $3,065,779$ & 822 & 548 & 274 & 377 & 123 & 308 & 247 & 468 \\
\hline $27 \%$ & $3,131,737$ & 822 & 548 & 274 & 377 & 123 & 308 & 247 & 468 \\
\hline $28 \%$ & $3,203,060$ & 822 & 548 & 274 & 377 & 123 & 308 & 247 & 468 \\
\hline $29 \%$ & $3,280,171$ & 822 & 548 & 274 & 377 & 123 & 308 & 247 & 468 \\
\hline $31 \%$ & $3,363,522$ & 822 & 548 & 274 & 377 & 123 & 308 & 247 & 468 \\
\hline $32 \%$ & $3,453,597$ & 822 & 548 & 274 & 377 & 123 & 308 & 247 & 468 \\
\hline $33 \%$ & $3,550,916$ & 822 & 548 & 274 & 377 & 123 & 308 & 247 & 468 \\
\hline $35 \%$ & $3,656,034$ & 822 & 548 & 274 & 377 & 123 & 308 & 247 & 468 \\
\hline $36 \%$ & $3,769,548$ & 822 & 548 & 274 & 377 & 123 & 308 & 247 & 468 \\
\hline $37 \%$ & $3,892,094$ & 822 & 548 & 274 & 377 & 123 & 308 & 247 & 468 \\
\hline $39 \%$ & $4,024,354$ & 822 & 548 & 274 & 377 & 123 & 308 & 247 & 468 \\
\hline $40 \%$ & $4,167,057$ & 822 & 548 & 274 & 377 & 123 & 308 & 247 & 468 \\
\hline
\end{tabular}


Table 6.15 presents the sensitivity analysis of the rate of increase in the Light crude price, which was conducted by varying the rate of increase between $1 \%$ and $40 \%$, and observing the changes in the optimum allocation decisions. At low rates of increase in the Light crude price, all the Light fields are placed on minimum operating rates. Since the Light crude fields represent the biggest portion of the portfolio, Field 20 (Heavy crude) in addition to all the Medium crude fields replaced the huge production loss of the Light crude fields. At a growth rate of $21 \%$ in the price of the Light, Field 12 becomes more profitable than Field 20. At a growth rate of $24 \%$ in the price of the Light, the optimum allocation decision is similar to the base case. The optimizer workflow (workflow), used to rank the fields, can be seen in the table above where Field 12 is the most profitable among the Light crude fields and Field 20 is the least profitable (among Light and Heavy crude fields) in the table. The production of Field 20 fell; this loss of production was compensated by Field 12.

Table 6.15: Light Price Growth Rate Optimization Sensitivity

\begin{tabular}{|c|c|c|c|c|c|c|c|c|c|c|c|c|c|c|}
\hline \multirow[b]{2}{*}{ Light_Price_Growth_Rate } & \multirow[b]{2}{*}{ Total_NPV } & \multicolumn{13}{|c|}{ Production Rate } \\
\hline & & Field 8 & Field 9 & Field 10 & Field 11 & Field 12 & Field 13 & Field 14 & Field 15 & Field 16 & Field 17 & Field 18 & Field 19 & Field 20 \\
\hline $1 \%$ & $2,759,540$ & 33 & 74 & 103 & 164 & 247 & 132 & 132 & 123 & 411 & 1027 & 822 & 822 & 619 \\
\hline $2 \%$ & $2,767,262$ & 33 & 74 & 103 & 164 & 247 & 132 & 132 & 123 & 411 & 1027 & 822 & 822 & 619 \\
\hline $4 \%$ & $2,775,612$ & 33 & 74 & 103 & 164 & 247 & 132 & 132 & 123 & 411 & 1027 & 822 & 822 & 619 \\
\hline $5 \%$ & $2,784,643$ & 33 & 74 & 103 & 164 & 247 & 132 & 132 & 123 & 411 & 1027 & 822 & 822 & 619 \\
\hline $6 \%$ & $2,794,412$ & 33 & 74 & 103 & 164 & 247 & 132 & 132 & 123 & 411 & 1027 & 822 & 822 & 619 \\
\hline $8 \%$ & $2,804,981$ & 33 & 74 & 103 & 164 & 247 & 132 & 132 & 123 & 411 & 1027 & 822 & 822 & 619 \\
\hline $9 \%$ & $2,816,418$ & 33 & 74 & 103 & 164 & 247 & 132 & 132 & 123 & 411 & 1027 & 822 & 822 & 619 \\
\hline $10 \%$ & $2,828,795$ & 33 & 74 & 103 & 164 & 247 & 132 & 132 & 123 & 411 & 1027 & 822 & 822 & 619 \\
\hline $12 \%$ & $2,842,190$ & 33 & 74 & 103 & 164 & 247 & 132 & 132 & 123 & 411 & 1027 & 822 & 822 & 619 \\
\hline $13 \%$ & $2,856,688$ & 33 & 74 & 103 & 164 & 247 & 132 & 132 & 123 & 411 & 1027 & 822 & 822 & 619 \\
\hline $14 \%$ & $2,872,381$ & 33 & 74 & 103 & 164 & 247 & 132 & 132 & 123 & 411 & 1027 & 822 & 822 & 619 \\
\hline $16 \%$ & $2,889,366$ & 33 & 74 & 103 & 164 & 247 & 132 & 132 & 123 & 411 & 1027 & 822 & 822 & 619 \\
\hline $17 \%$ & $2,907,750$ & 33 & 74 & 103 & 164 & 247 & 132 & 132 & 123 & 411 & 1027 & 822 & 822 & 619 \\
\hline $18 \%$ & $2,927,647$ & 33 & 74 & 103 & 164 & 247 & 132 & 132 & 123 & 411 & 1027 & 822 & 822 & 619 \\
\hline $20 \%$ & $2,949,181$ & 33 & 74 & 103 & 164 & 247 & 132 & 132 & 123 & 411 & 1027 & 822 & 822 & 619 \\
\hline $21 \%$ & $2,975,074$ & 33 & 74 & 103 & 164 & 660 & 132 & 132 & 123 & 411 & 1027 & 822 & 822 & 205 \\
\hline $23 \%$ & $3,010,648$ & 33 & 74 & 103 & 164 & 660 & 132 & 132 & 123 & 411 & 1027 & 822 & 822 & 205 \\
\hline $24 \%$ & $3,098,304$ & 110 & 247 & 342 & 548 & 822 & 438 & 438 & 411 & 123 & 308 & 247 & 468 & 205 \\
\hline $25 \%$ & $3,196,666$ & 110 & 247 & 342 & 548 & 822 & 438 & 438 & 411 & 123 & 308 & 247 & 468 & 205 \\
\hline $27 \%$ & $3,303,049$ & 110 & 247 & 342 & 548 & 822 & 438 & 438 & 411 & 123 & 308 & 247 & 468 & 205 \\
\hline $28 \%$ & $3,418,088$ & 110 & 247 & 342 & 548 & 822 & 438 & 438 & 411 & 123 & 308 & 247 & 468 & 205 \\
\hline $29 \%$ & $3,542,461$ & 110 & 247 & 342 & 548 & 822 & 438 & 438 & 411 & 123 & 308 & 247 & 468 & 205 \\
\hline $31 \%$ & $3,676,899$ & 110 & 247 & 342 & 548 & 822 & 438 & 438 & 411 & 123 & 308 & 247 & 468 & 205 \\
\hline $32 \%$ & $3,822,182$ & 110 & 247 & 342 & 548 & 822 & 438 & 438 & 411 & 123 & 308 & 247 & 468 & 205 \\
\hline $33 \%$ & $3,979,148$ & 110 & 247 & 342 & 548 & 822 & 438 & 438 & 411 & 123 & 308 & 247 & 468 & 205 \\
\hline $35 \%$ & $4,148,695$ & 110 & 247 & 342 & 548 & 822 & 438 & 438 & 411 & 123 & 308 & 247 & 468 & 205 \\
\hline $36 \%$ & $4,331,783$ & 110 & 247 & 342 & 548 & 822 & 438 & 438 & 411 & 123 & 308 & 247 & 468 & 205 \\
\hline $37 \%$ & $4,529,439$ & 110 & 247 & 342 & 548 & 822 & 438 & 438 & 411 & 123 & 308 & 247 & 468 & 205 \\
\hline $39 \%$ & $4,742,763$ & 110 & 247 & 342 & 548 & 822 & 438 & 438 & 411 & 123 & 308 & 247 & 468 & 205 \\
\hline $40 \%$ & $4,972,930$ & 110 & 247 & 342 & 548 & 822 & 438 & 438 & 411 & 123 & 308 & 247 & 468 & 205 \\
\hline
\end{tabular}


The sensitivity analysis of the rate of the Medium price increases is shown in Table 6.16 and is conducted by varying the rate of increase between $1 \%$ and $40 \%$, and observing the changes in the optimum allocation decisions. At low rates of the Light crude price increases, the Medium fields are placed on minimum operating rates. At a growth rate of $23 \%$ in the price of the Medium crude, which is used in the study, the excess production rate of Field 20 is assigned back to Field 19, and the optimum decision is the base case decision. When the rate of the Medium crude price increases is $27 \%$, all the Medium crude fields produce at maximum potential while the Light crude productions are restricted to meet the corporate total rate objective. Field 12 is the highest Light crude Field value since it is the only Light crude field that is producing above the minimum potential.

Table 6.16: Medium Price Growth Rate Optimization Sensitivity

\begin{tabular}{|c|c|c|c|c|c|c|c|c|c|c|c|c|c|c|}
\hline \multirow[b]{2}{*}{ Medium_Price_Growth_Rate } & \multirow[b]{2}{*}{ Total_NPV } & \multicolumn{13}{|c|}{ Production Rate } \\
\hline & & Field 8 & Field 9 & Field 10 & Field 11 & Field 12 & Field 13 & Field 14 & Field 15 & Field 16 & Field 17 & Field 18 & Field 19 & Field 20 \\
\hline $1 \%$ & $2,923,403$ & 110 & 247 & 342 & 548 & 822 & 438 & 438 & 411 & 123 & 308 & 247 & 247 & 427 \\
\hline $2 \%$ & $2,930,216$ & 110 & 247 & 342 & 548 & 822 & 438 & 438 & 411 & 123 & 308 & 247 & 247 & 427 \\
\hline $4 \%$ & $2,937,583$ & 110 & 247 & 342 & 548 & 822 & 438 & 438 & 411 & 123 & 308 & 247 & 247 & 427 \\
\hline $5 \%$ & $2,945,551$ & 110 & 247 & 342 & 548 & 822 & 438 & 438 & 411 & 123 & 308 & 247 & 247 & 427 \\
\hline $6 \%$ & $2,954,170$ & 110 & 247 & 342 & 548 & 822 & 438 & 438 & 411 & 123 & 308 & 247 & 247 & 427 \\
\hline $8 \%$ & $2,963,495$ & 110 & 247 & 342 & 548 & 822 & 438 & 438 & 411 & 123 & 308 & 247 & 247 & 427 \\
\hline $9 \%$ & $2,973,585$ & 110 & 247 & 342 & 548 & 822 & 438 & 438 & 411 & 123 & 308 & 247 & 247 & 427 \\
\hline $10 \%$ & $2,984,505$ & 110 & 247 & 342 & 548 & 822 & 438 & 438 & 411 & 123 & 308 & 247 & 247 & 427 \\
\hline $12 \%$ & $2,996,323$ & 110 & 247 & 342 & 548 & 822 & 438 & 438 & 411 & 123 & 308 & 247 & 247 & 427 \\
\hline $13 \%$ & $3,009,114$ & 110 & 247 & 342 & 548 & 822 & 438 & 438 & 411 & 123 & 308 & 247 & 247 & 427 \\
\hline $14 \%$ & $3,022,960$ & 110 & 247 & 342 & 548 & 822 & 438 & 438 & 411 & 123 & 308 & 247 & 247 & 427 \\
\hline $16 \%$ & $3,037,945$ & 110 & 247 & 342 & 548 & 822 & 438 & 438 & 411 & 123 & 308 & 247 & 247 & 427 \\
\hline $17 \%$ & $3,054,165$ & 110 & 247 & 342 & 548 & 822 & 438 & 438 & 411 & 123 & 308 & 247 & 247 & 427 \\
\hline $18 \%$ & $3,071,720$ & 110 & 247 & 342 & 548 & 822 & 438 & 438 & 411 & 123 & 308 & 247 & 247 & 427 \\
\hline $20 \%$ & $3,090,719$ & 110 & 247 & 342 & 548 & 822 & 438 & 438 & 411 & 123 & 308 & 247 & 247 & 427 \\
\hline $21 \%$ & $3,111,278$ & 110 & 247 & 342 & 548 & 822 & 438 & 438 & 411 & 123 & 308 & 247 & 247 & 427 \\
\hline $23 \%$ & $3,137,276$ & 110 & 247 & 342 & 548 & 822 & 438 & 438 & 411 & 123 & 308 & 247 & 468 & 205 \\
\hline $24 \%$ & $3,167,119$ & 110 & 247 & 342 & 548 & 822 & 438 & 438 & 411 & 123 & 308 & 247 & 468 & 205 \\
\hline $25 \%$ & $3,199,730$ & 33 & 247 & 342 & 548 & 822 & 438 & 438 & 411 & 123 & 308 & 247 & 545 & 205 \\
\hline $27 \%$ & $3,286,592$ & 33 & 74 & 103 & 164 & 660 & 132 & 132 & 123 & 411 & 1027 & 822 & 822 & 205 \\
\hline $28 \%$ & $3,388,088$ & 33 & 74 & 103 & 164 & 660 & 132 & 132 & 123 & 411 & 1027 & 822 & 822 & 205 \\
\hline $29 \%$ & $3,497,820$ & 33 & 74 & 103 & 164 & 660 & 132 & 132 & 123 & 411 & 1027 & 822 & 822 & 205 \\
\hline $31 \%$ & $3,616,431$ & 33 & 74 & 103 & 164 & 660 & 132 & 132 & 123 & 411 & 1027 & 822 & 822 & 205 \\
\hline $32 \%$ & $3,744,610$ & 33 & 74 & 103 & 164 & 660 & 132 & 132 & 123 & 411 & 1027 & 822 & 822 & 205 \\
\hline $33 \%$ & $3,883,098$ & 33 & 74 & 103 & 164 & 660 & 132 & 132 & 123 & 411 & 1027 & 822 & 822 & 205 \\
\hline $35 \%$ & $4,032,685$ & 33 & 74 & 103 & 164 & 660 & 132 & 132 & 123 & 411 & 1027 & 822 & 822 & 205 \\
\hline $36 \%$ & $4,194,219$ & 33 & 74 & 103 & 164 & 660 & 132 & 132 & 123 & 411 & 1027 & 822 & 822 & 205 \\
\hline $37 \%$ & $4,368,606$ & 33 & 74 & 103 & 164 & 660 & 132 & 132 & 123 & 411 & 1027 & 822 & 822 & 205 \\
\hline $39 \%$ & $4,556,817$ & 33 & 74 & 103 & 164 & 660 & 132 & 132 & 123 & 411 & 1027 & 822 & 822 & 205 \\
\hline $40 \%$ & $4,759,887$ & 33 & 74 & 103 & 164 & 660 & 132 & 132 & 123 & 411 & 1027 & 822 & 822 & 205 \\
\hline
\end{tabular}


The sensitivity analysis of the rate of the Heavy price increases is shown in Table 6.17 and is conducted by varying the rate of increase between $1 \%$ and $40 \%$, and observing the changes in the optimum allocation decisions. Initially, the production rates of all the Heavy crude fields are placed at minimum operating rates. At a growth rate of $24 \%$ in the price of Heavy crude, Field 20 (Heavy crude) is more profitable than Field 19 (Medium crude). These deviations in the allocation decision from the base case decision reveals the sensitivity of the optimum allocation decision to the rate of increase in the price of Heavy crude. As shown in the table, only $2 \%$ change in the Heavy price growth rate, from $22 \%$ to $24 \%$, caused major changes in the optimum allocation decision. At a rate of the Heavy price increases of $27 \%$, all the Heavy fields are placed at maximum rates. Similar to the previous crude grades, the workflow used by the optimizer to rank the field in the optimum allocation decision is clear in the table. Field 20 is the highest Heavy crude field because it is the first field, among the Heavy crude fields, whose production rate is increased. 
Table 6.17: Heavy Price Growth Rate Optimization Sensitivity

\begin{tabular}{|c|c|c|c|c|c|c|c|c|c|c|c|c|c|c|}
\hline \multirow[b]{2}{*}{ Medium_Price_Growth_Rate } & \multirow[b]{2}{*}{ Total_NPV } & \multicolumn{13}{|c|}{ Production Rate } \\
\hline & & Field 8 & Field 9 & Field 10 & Field 11 & Field 12 & Field 13 & Field 14 & Field 15 & Field 19 & Field 20 & Field 21 & Field 22 & Field 23 \\
\hline $1 \%$ & $2,986,481$ & 110 & 247 & 342 & 548 & 822 & 438 & 438 & 411 & 468 & 205 & 222 & 197 & 164 \\
\hline $2 \%$ & $2,992,119$ & 110 & 247 & 342 & 548 & 822 & 438 & 438 & 411 & 468 & 205 & 222 & 197 & 164 \\
\hline $4 \%$ & $2,998,216$ & 110 & 247 & 342 & 548 & 822 & 438 & 438 & 411 & 468 & 205 & 222 & 197 & 164 \\
\hline $5 \%$ & $3,004,810$ & 110 & 247 & 342 & 548 & 822 & 438 & 438 & 411 & 468 & 205 & 222 & 197 & 164 \\
\hline $6 \%$ & $3,011,943$ & 110 & 247 & 342 & 548 & 822 & 438 & 438 & 411 & 468 & 205 & 222 & 197 & 164 \\
\hline $8 \%$ & $3,019,660$ & 110 & 247 & 342 & 548 & 822 & 438 & 438 & 411 & 468 & 205 & 222 & 197 & 164 \\
\hline $9 \%$ & $3,028,010$ & 110 & 247 & 342 & 548 & 822 & 438 & 438 & 411 & 468 & 205 & 222 & 197 & 164 \\
\hline $10 \%$ & $3,037,047$ & 110 & 247 & 342 & 548 & 822 & 438 & 438 & 411 & 468 & 205 & 222 & 197 & 164 \\
\hline $12 \%$ & $3,046,827$ & 110 & 247 & 342 & 548 & 822 & 438 & 438 & 411 & 468 & 205 & 222 & 197 & 164 \\
\hline $13 \%$ & $3,057,413$ & 110 & 247 & 342 & 548 & 822 & 438 & 438 & 411 & 468 & 205 & 222 & 197 & 164 \\
\hline $14 \%$ & $3,068,870$ & 110 & 247 & 342 & 548 & 822 & 438 & 438 & 411 & 468 & 205 & 222 & 197 & 164 \\
\hline $16 \%$ & $3,081,272$ & 110 & 247 & 342 & 548 & 822 & 438 & 438 & 411 & 468 & 205 & 222 & 197 & 164 \\
\hline $17 \%$ & $3,094,695$ & 110 & 247 & 342 & 548 & 822 & 438 & 438 & 411 & 468 & 205 & 222 & 197 & 164 \\
\hline $18 \%$ & $3,109,222$ & 110 & 247 & 342 & 548 & 822 & 438 & 438 & 411 & 468 & 205 & 222 & 197 & 164 \\
\hline $20 \%$ & $3,124,945$ & 110 & 247 & 342 & 548 & 822 & 438 & 438 & 411 & 468 & 205 & 222 & 197 & 164 \\
\hline $21 \%$ & $3,141,959$ & 110 & 247 & 342 & 548 & 822 & 438 & 438 & 411 & 468 & 205 & 222 & 197 & 164 \\
\hline $23 \%$ & $3,160,369$ & 110 & 247 & 342 & 548 & 822 & 438 & 438 & 411 & 468 & 205 & 222 & 197 & 164 \\
\hline $24 \%$ & $3,180,266$ & 110 & 247 & 342 & 548 & 822 & 438 & 438 & 411 & 247 & 427 & 222 & 197 & 164 \\
\hline $25 \%$ & $3,207,871$ & 110 & 247 & 342 & 548 & 822 & 438 & 438 & 411 & 247 & 427 & 222 & 197 & 164 \\
\hline $27 \%$ & $3,260,970$ & 33 & 74 & 103 & 319 & 822 & 132 & 132 & 123 & 247 & 685 & 740 & 658 & 548 \\
\hline $28 \%$ & $3,344,964$ & 33 & 74 & 103 & 319 & 822 & 132 & 132 & 123 & 247 & 685 & 740 & 658 & 548 \\
\hline $29 \%$ & $3,435,773$ & 33 & 74 & 103 & 319 & 822 & 132 & 132 & 123 & 247 & 685 & 740 & 658 & 548 \\
\hline $31 \%$ & $3,533,931$ & 33 & 74 & 103 & 319 & 822 & 132 & 132 & 123 & 247 & 685 & 740 & 658 & 548 \\
\hline $32 \%$ & $3,640,007$ & 33 & 74 & 103 & 319 & 822 & 132 & 132 & 123 & 247 & 685 & 740 & 658 & 548 \\
\hline $33 \%$ & $3,754,614$ & 33 & 74 & 103 & 319 & 822 & 132 & 132 & 123 & 247 & 685 & 740 & 658 & 548 \\
\hline $35 \%$ & $3,878,406$ & 33 & 74 & 103 & 319 & 822 & 132 & 132 & 123 & 247 & 685 & 740 & 658 & 548 \\
\hline $36 \%$ & $4,012,085$ & 33 & 74 & 103 & 319 & 822 & 132 & 132 & 123 & 247 & 685 & 740 & 658 & 548 \\
\hline $37 \%$ & $4,156,401$ & 33 & 74 & 103 & 319 & 822 & 132 & 132 & 123 & 247 & 685 & 740 & 658 & 548 \\
\hline $39 \%$ & $4,312,156$ & 33 & 74 & 103 & 319 & 822 & 132 & 132 & 123 & 247 & 685 & 740 & 658 & 548 \\
\hline $40 \%$ & $4,480,209$ & 33 & 74 & 103 & 319 & 822 & 132 & 132 & 123 & 247 & 685 & 740 & 658 & 548 \\
\hline
\end{tabular}

\subsection{The Probabilistic Price Models}

The Probabilistic approach involves probability distribution and a stochastic process to account for price volatility in the decision making. In this section, the results of four commonly used stochastic processes are presented and analyzed. The four stochastic processes are Independent Log-normal Distribution (ILD), Geometric Brownian Motion (GBM), Mean Reversion Model (MRM), and Mean Reversion with Jumps (MRMJ). The modeling processes of these four models are discussed in the section on Price Modeling.

\subsubsection{The Independent Log-nORmal Distribution Price Model (ILD)}

The ILD is the simplest stochastic price forecast model; the prices are sampled from the log-normal distribution of each time period. The model was modified to account 
for the increasing volatility with time by imposing an increasing standard deviation of the log-normal distribution with time. The forecast formulation process is discussed in the section on Price Formulation. Figure 6.9 shows a 200-iteration sample of the ILD model forecast; the increasing volatility with time is clear.

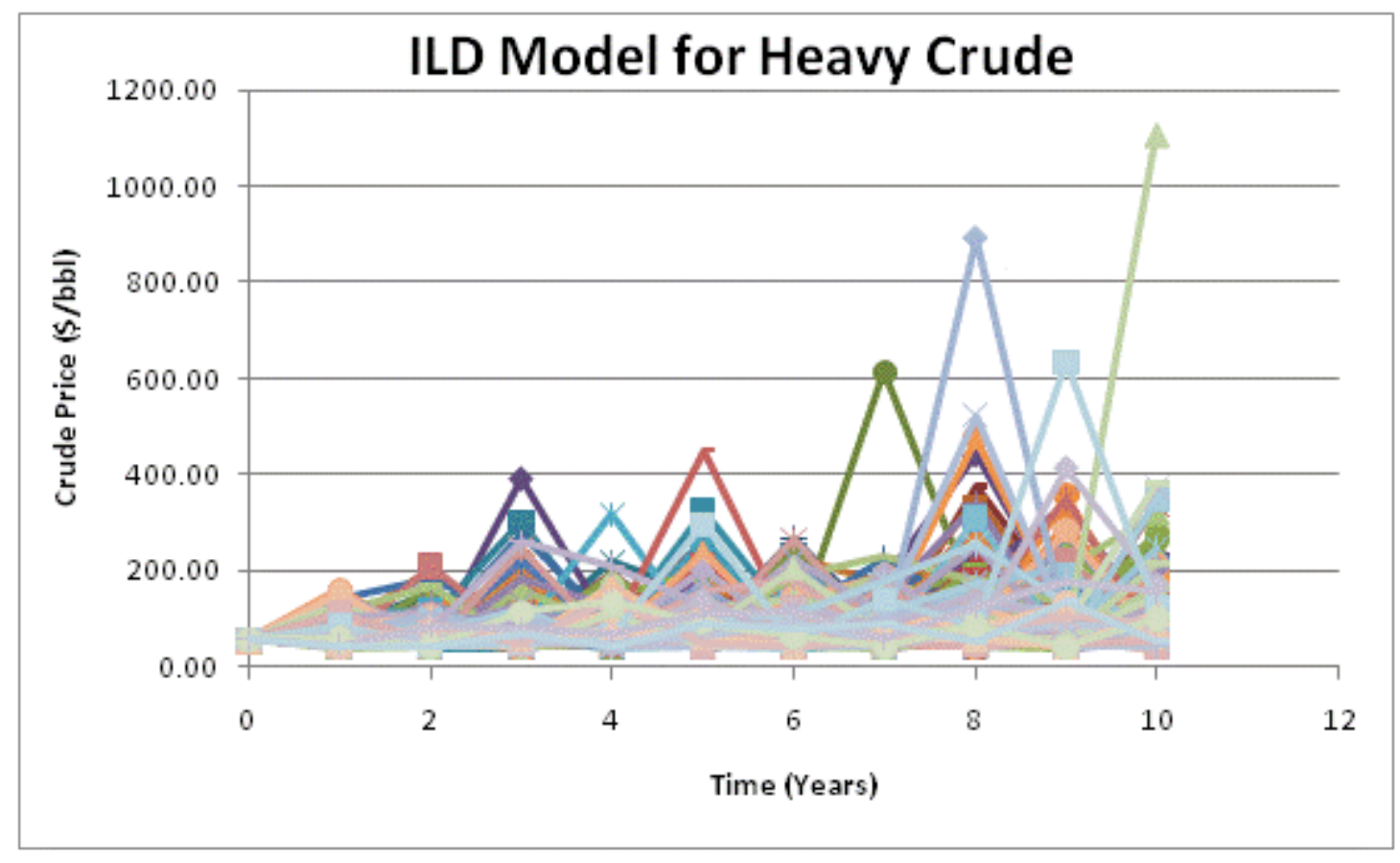

Figure 6.9: The ILD Price Forecast Model (200 iterations)

\subsubsection{The Optimum Decision of the ILD Forecast Model}

Table 6.18 and Figure 6.10 show the optimum production allocation decision when using the Floating Price model with a mean of total NPV of MM\$ 809,072 and a standard deviation of MM\$ 186,918 . The mean of the total NPV distribution is close to the total NPV (MM\$ 720,306) of the Flat Price model. The standard deviation reveals the high uncertainty, which can be seen in Figure 6.11 where the long tail shows the possibility of total NPV reaching MM\$2,000,000. In other words, the range of the total NPV is between MM\$500,000 and 2,000,000. Moreover, the optimum allocation 
decision is similar to that of the Flat Price model; this means that the ILD price model has a significant impact on the value of the total NPV, but not on the optimum production allocation decision. The optimizer uses a workflow (a workflow to rank the field production), similar to that used in the Flat Price model to allocate production rates among different fields and crudes while meeting the company's total target rate (functionality requirement). Table 6.19 shows the optimum production allocation by crude grade, which reflects the company's resources; the ratio of the resources can be realized from the relatively small XXLight production rate. In other words, the XXLight fields are depleted due to the fact that the XXLight crude is the "easy oil" and has been produced over the last decades.

Table 6.18: The Optimum Allocation for ILD Price Forecast Model

\begin{tabular}{|c|c|c|c|c|}
\hline Field & $\begin{array}{l}\text { Optimum } \\
\text { Production Rate } \\
\text { (MBD) }\end{array}$ & $\begin{array}{l}\text { Production } \\
\text { Rate Status }\end{array}$ & $\begin{array}{l}\text { "Flat Price" } \\
\text { Optimum } \\
\text { Production } \\
\text { Rate } \\
\end{array}$ & $\begin{array}{c}\text { Difference } \\
\text { Between } \\
\text { Current and } \\
\text { Flat Price Case } \\
\end{array}$ \\
\hline 1 & 153 & Upper Bound & 153 & 0 \\
\hline 2 & 329 & Upper Bound & 329 & 0 \\
\hline 3 & 205 & Upper Bound & 205 & 0 \\
\hline 4 & 822 & Upper Bound & 822 & 0 \\
\hline 5 & 548 & Upper Bound & 548 & 0 \\
\hline 6 & 274 & Upper Bound & 274 & 0 \\
\hline 7 & 377 & Upper Bound & 377 & 0 \\
\hline 8 & 33 & Lower_Bound & 33 & 0 \\
\hline 9 & 247 & Upper Bound & 247 & 0 \\
\hline 10 & 342 & Upper Bound & 342 & 0 \\
\hline 11 & 548 & Upper Bound & 548 & 0 \\
\hline 12 & 822 & Upper Bound & 822 & 0 \\
\hline 13 & 438 & Upper Bound & 438 & 0 \\
\hline 14 & 438 & Upper Bound & 438 & 0 \\
\hline 15 & 411 & Upper Bound & 411 & 0 \\
\hline 16 & 123 & Lower_Bound & 123 & 0 \\
\hline 17 & 308 & Lower_Bound & 308 & 0 \\
\hline 18 & 247 & Lower_Bound & 247 & 0 \\
\hline 19 & 545 & & 545 & 0 \\
\hline 20 & 205 & Lower_Bound & 205 & 0 \\
\hline 21 & 222 & Lower_Bound & 222 & 0 \\
\hline 22 & 197 & Lower_Bound & 197 & 0 \\
\hline 23 & 164 & Lower_Bound & 164 & 0 \\
\hline
\end{tabular}


Table 6.19: The Optimum Allocation by Crude Grade for the ILD Price Forecast Model

\begin{tabular}{|lc|}
\hline Crude Type & $\begin{array}{c}\text { Optimal Production } \\
\text { Rate (MBD) }\end{array}$ \\
\cline { 2 - 2 } XXL & 688 \\
XI & 2,021 \\
AL & 3,279 \\
AM & 1,223 \\
AH & 789 \\
sum & 8,000 \\
\hline
\end{tabular}

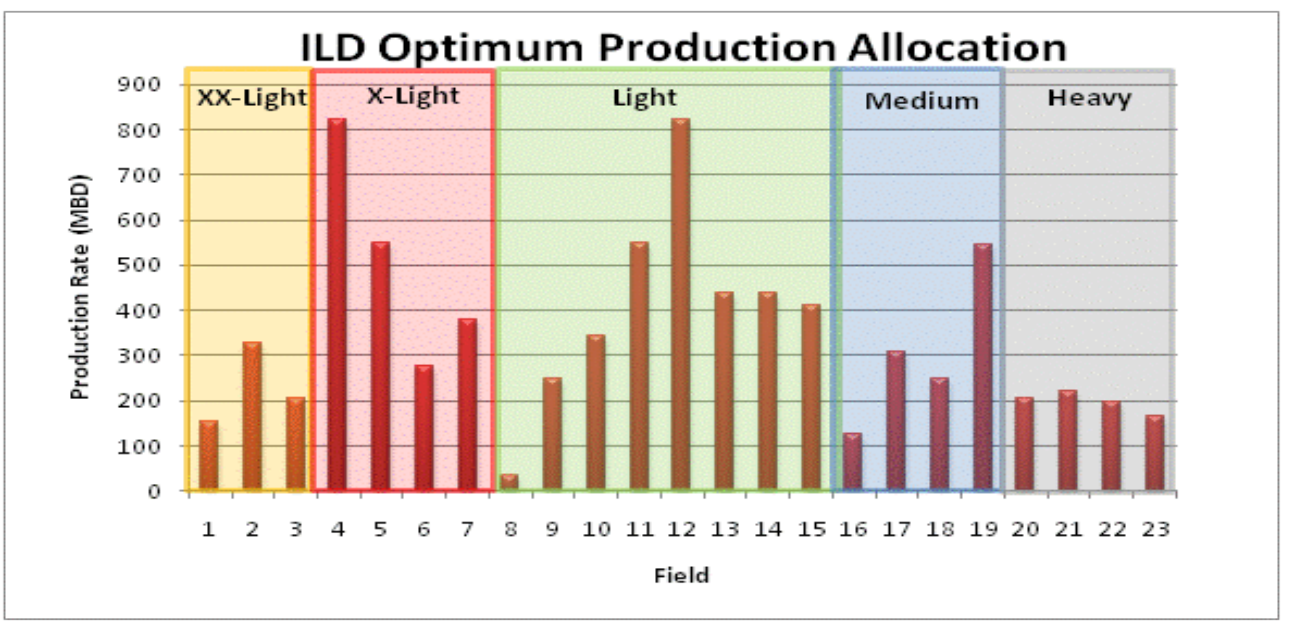

Figure 6.10: Optimum Production Allocation for the ILD Price Forecast Model

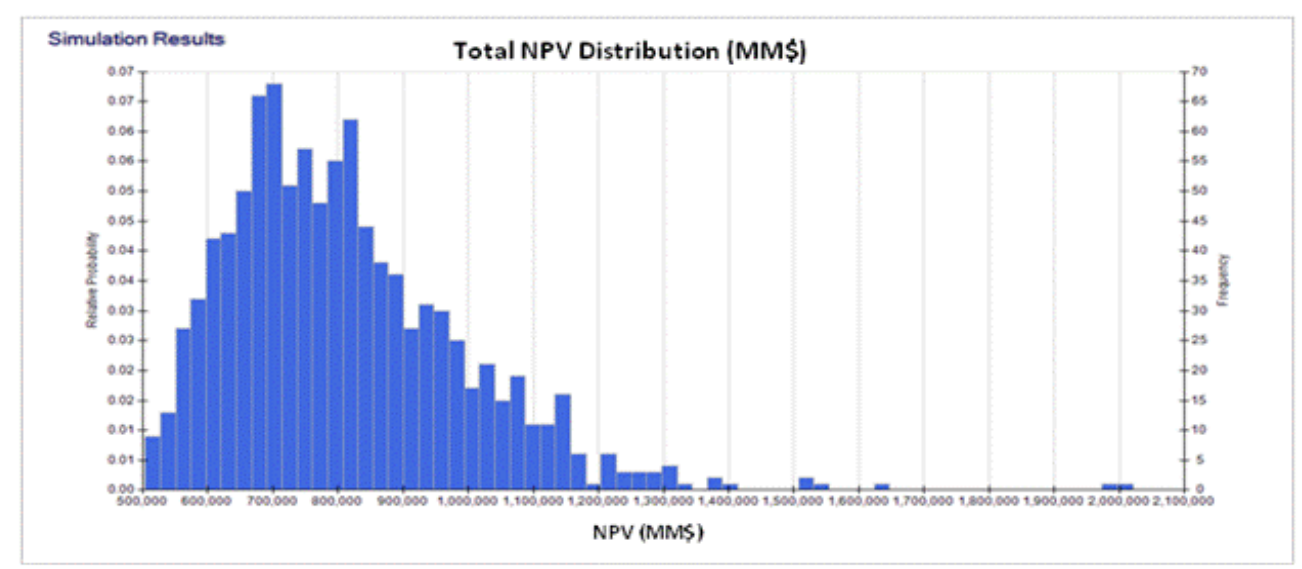

Figure 6.11: Total NPV Probability Density Function Resulted from the ILD Price Forecast Model 


\subsubsection{The Sensitivity Analysis of the ILD Forecast Model}

Here, we identify the most significant parameters on the total NPV and how these parameters affect the optimum allocation decision. Figure 6.12 is the tornado chart for the total NPV that is generated by varying the parameters, required for NPV calculations, by $\pm 10 \%$. Each parameter was analyzed individually to observe its affect on the total NPV. The tornado chart reveals the significance of price correlation assumptions. The tornado chart is generated by sampling an iteration of the uncertain variables (uncertain variables are fixed) and varying the other parameters to investigate their impact on the total NPV. For example, the crude prices, in the probabilistic approach, are sampled once in each time period; then a parameter (e.g., standard deviation) is varied by $\pm 10 \%$. The ILD model is implemented to model the prices of the Heavy crude; then, the prices of the remaining crude grades are correlated to the Heavy crude prices as shown in the section on correlating the crude prices. It is not feasible to analyze the effect of the ILD modeling parameters on the optimum allocation decision since only the Heavy crude prices are modeled using the ILD model. In other words, the ILD model does not impact the relationship between the prices of different crudes. Any change in the Heavy crude prices, resulted from modifying a price modeling parameter, is correlated to the prices of the remaining crudes hence the optimum allocation decision is affected. The impacts of the initial Heavy crude price, initial Heavy crude S.D., and S.D. growth factor on the mean of the total NPV and on the optimum allocation decision were investigated. The three parameters have a significant impact on the value of the mean of the total NPV as shown in Figures 6.13, 6.14, and 6.15, but they do not impact the optimum decision because the prices are correlated to the Heavy crude prices. In this section, the impacts of price correlation parameters (the slope of the regression correlation) on the optimum allocation decision are presented. 


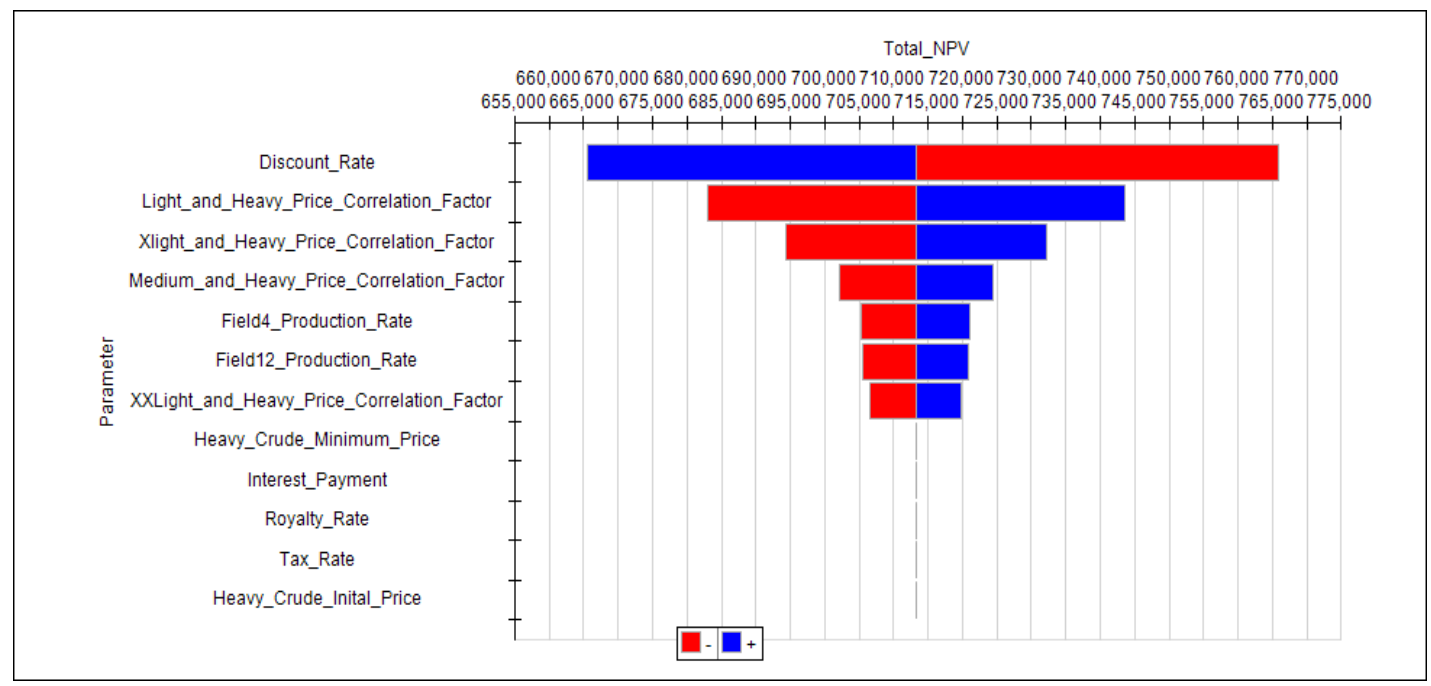

Figure 6.12: Total NPV Tornado Chart by Varying Parameters $\pm 10 \%$

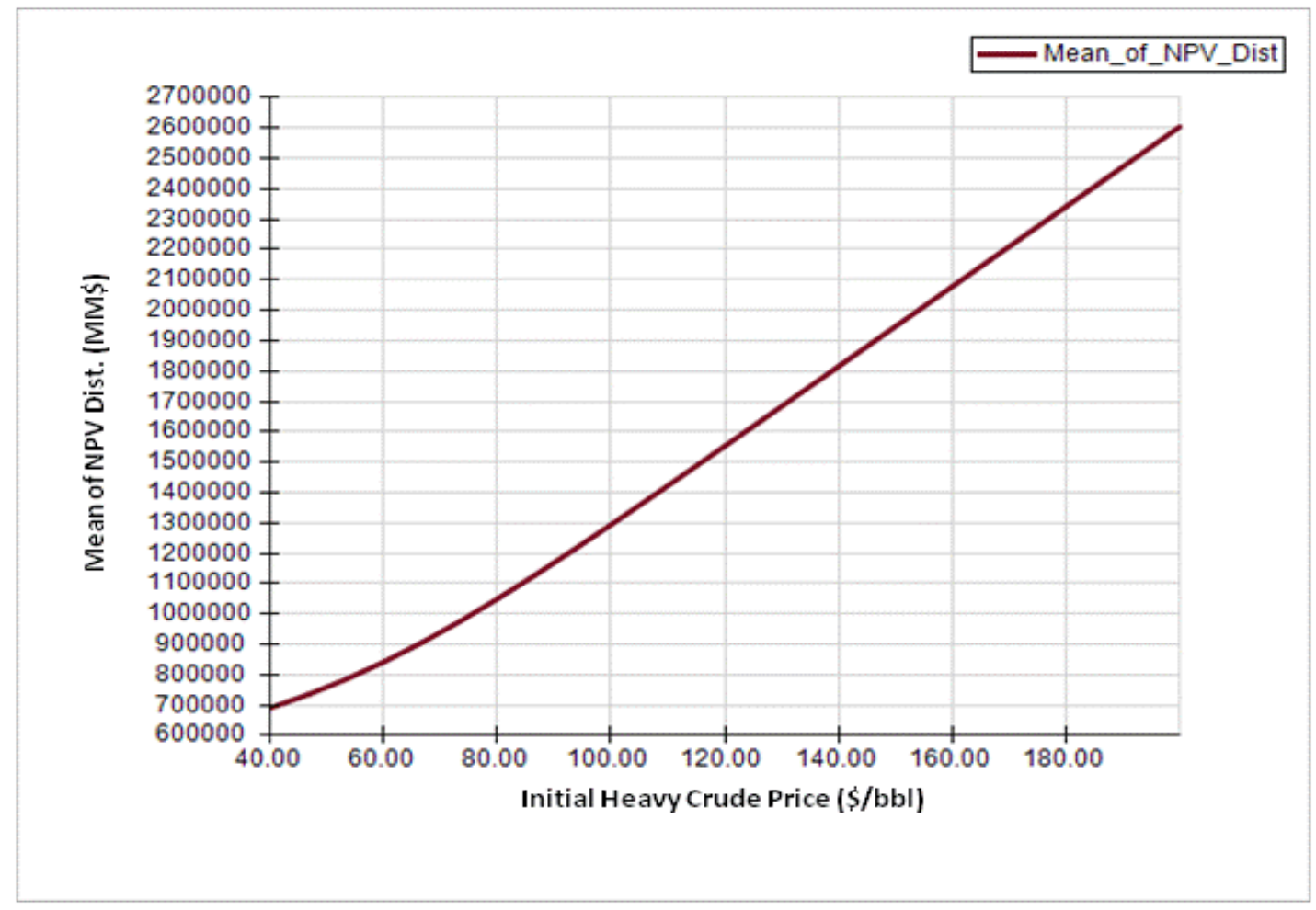

Figure 6.13: Sensitivity Analysis of Mean of Total NPV by Changing the Initial Heavy Crude Price 


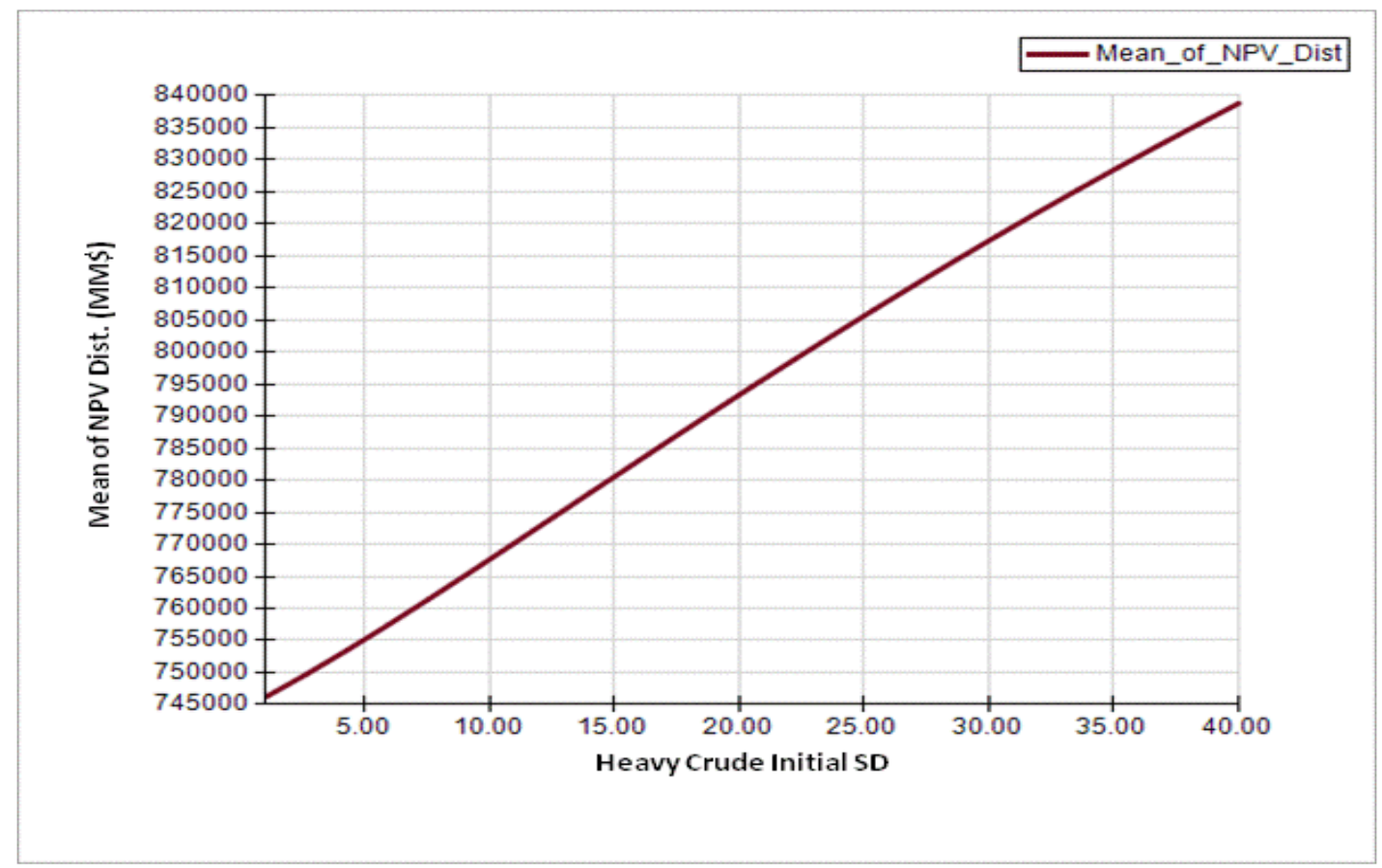

Figure 6.14: Sensitivity Analysis of Mean of Total NPV by Changing the Initial Heavy Crude Standard Deviation.

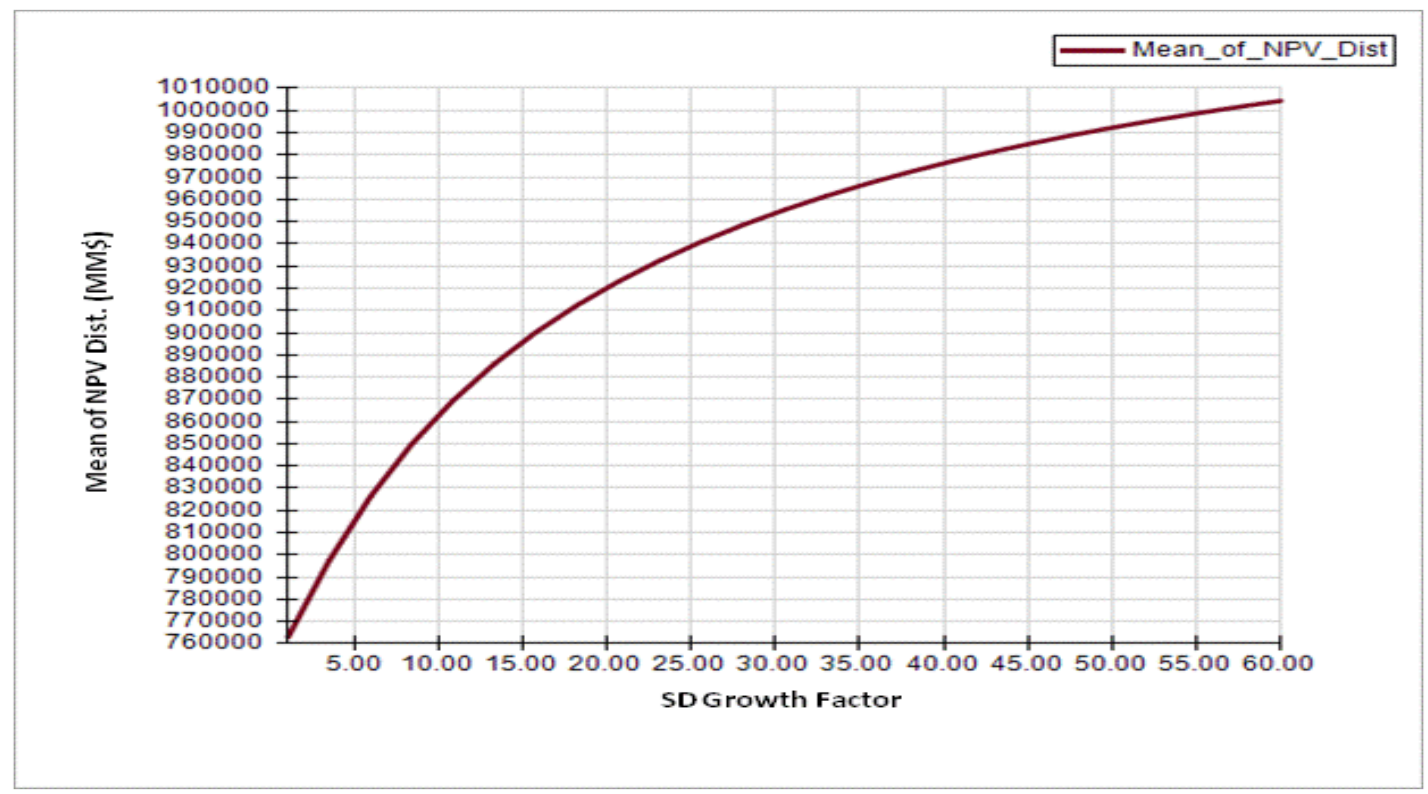

Figure 6.14: Sensitivity Analysis of Mean of Total NPV by Changing the Standard Deviation Growth Factor 
Table 6.20 shows the optimum allocation decisions as the Medium-Heavy Price Correlation Factor is varied between 0.10 and 1.06. The correlation factor is the slope in the regression correlation equation presented in the price correlation section. The table shows only the fields that are impacted by changing the correlation factor. The table shows the impact of the correlation factor; the highlighted correlation factors are those where a change in the optimum allocation decision occurs. As the Medium-Heavy correlation factor decreases, reducing the value of the Medium crude price, the production rates allocated to the Medium crude fields also decreases. The optimizer uses a workflow to allocate the production rate between the fields. For a price correlation factor of 0.98 and lower, all the Medium crude fields are placed at minimum operating levels. Similar to the outcome of the deterministic forecast approaches, Field 19 is the most valuable field among the Medium ones since it is the first to receive an increase in the allocated rate when the Medium crude becomes more valuable than the Heavy crude. At price correlation factors of 1.05 and 1.06 , the production rates are re-allocated from the Light crude to the Medium crude fields. This re-allocation placed the Medium crude filed at maximum rates.

Table 6.20: Optimization Sensitivity Analysis of the Medium-Heavy Price Correlation Factor

\begin{tabular}{|c|c|c|c|c|c|c|c|c|c|c|c|c|c|}
\hline \multicolumn{14}{|c|}{ Production Rate } \\
\hline \multirow{2}{*}{ Medium-Heavy Price Correlation Factor } & \multicolumn{8}{|c|}{ Light } & \multicolumn{4}{|c|}{ Medium } & \multirow{2}{*}{$\begin{array}{l}\text { Heavy } \\
\text { Field } 20\end{array}$} \\
\hline & Field 8 & Field 9 & Field 10 & Field 11 & Field 12 & Field 13 & Field 14 & Field 15 & Field 16 & Field 17 & Field 18 & Field 19 & \\
\hline 0.10 & 33 & 247 & 342 & 548 & 822 & 438 & 438 & 411 & 123 & 308 & 247 & 247 & 504 \\
\hline 0.80 & 33 & 247 & 342 & 548 & 822 & 438 & 438 & 411 & 123 & 308 & 247 & 247 & 504 \\
\hline 0.98 & 33 & 247 & 342 & 548 & 822 & 438 & 438 & 411 & 123 & 308 & 247 & 247 & 504 \\
\hline 0.99 & 33 & 247 & 342 & 548 & 822 & 438 & 438 & 411 & 123 & 308 & 247 & 545 & 205 \\
\hline 1.01 & 33 & 247 & 342 & 548 & 822 & 438 & 438 & 411 & 123 & 308 & 247 & 545 & 205 \\
\hline 1.04 & 33 & 247 & 342 & 548 & 822 & 438 & 438 & 411 & 123 & 308 & 247 & 545 & 205 \\
\hline 1.05 & 33 & 74 & 103 & 548 & 822 & 132 & 438 & 123 & 123 & 463 & 822 & 822 & 205 \\
\hline 1.06 & 33 & 74 & 103 & 164 & 660 & 132 & 132 & 123 & 411 & 1027 & 822 & 822 & 205 \\
\hline
\end{tabular}


Table 6.21 shows the optimum allocation decisions as the Light-Heavy Price Correlation Factor varies. The correlation factor is the slope in the regression correlation equation presented in the price correlation section. The table shows only the fields that are impacted by changing the correlation factor. The highlighted correlation factors are those where a change in the optimum allocation decision occurs. As the Light-Heavy correlation factor decreases, reducing the value of the Light crude, the production rates allocated to the Light crude fields also decreases. The optimizer uses a workflow to allocate the production rate between the fields. The changes in the optimum allocation decision can be seen at the 1.0 and 1.08 correlation factor.

Table 6.21: Optimization Sensitivity Analysis of the Light-Heavy Price Correlation Factor

\begin{tabular}{|c|c|c|c|c|c|c|c|c|c|c|c|c|c|}
\hline \multicolumn{14}{|c|}{$\begin{array}{l}\text { Production Rate } \\
\text { Prots }\end{array}$} \\
\hline \multirow{2}{*}{ Light-Heavy Price Correlation Factor } & \multicolumn{8}{|c|}{ Light } & \multicolumn{4}{|c|}{ Medium } & \multirow{2}{*}{$\begin{array}{l}\text { Heavy } \\
\text { Field } 20\end{array}$} \\
\hline & Field 8 & Field 9 & Field 10 & Field 11 & Field 12 & Field 13 & Field 14 & Field 15 & Field 16 & Field 17 & Field 18 & Field 19 & \\
\hline 0.05 & 33 & 74 & 103 & 164 & 247 & 132 & 132 & 123 & 411 & 1027 & 822 & 822 & 619 \\
\hline 0.10 & 33 & 74 & 103 & 164 & 247 & 132 & 132 & 123 & 411 & 1027 & 822 & 822 & 619 \\
\hline 0.50 & 33 & 74 & 103 & 164 & 247 & 132 & 132 & 123 & 411 & 1027 & 822 & 822 & 619 \\
\hline 0.80 & 33 & 74 & 103 & 164 & 247 & 132 & 132 & 123 & 411 & 1027 & 822 & 822 & 619 \\
\hline 0.95 & 33 & 74 & 103 & 164 & 247 & 132 & 132 & 123 & 411 & 1027 & 822 & 822 & 619 \\
\hline 1.00 & 33 & 247 & 342 & 548 & 822 & 438 & 438 & 411 & 123 & 308 & 247 & 545 & 205 \\
\hline 1.05 & 33 & 247 & 342 & 548 & 822 & 438 & 438 & 411 & 123 & 308 & 247 & 545 & 205 \\
\hline 1.07 & 33 & 247 & 342 & 548 & 822 & 438 & 438 & 411 & 123 & 308 & 247 & 545 & 205 \\
\hline 1.08 & 110 & 247 & 342 & 548 & 822 & 438 & 438 & 411 & 123 & 308 & 247 & 468 & 205 \\
\hline
\end{tabular}

Table 6.22 shows the optimum allocation decisions as the XLight-Heavy Price Correlation Factor varies. The correlation factor is the slope in the regression correlation equation presented in the section on Correlating the Crude Prices. The table shows only the fields that are impacted by changing the correlation factor. The highlighted correlation factors are those where a change in the optimum allocation decision occurs. As the XLight-Heavy correlation factor decreases, reducing the value of the XLight crude, the production rates allocated to the XLight crude fields also decreases. The optimizer uses a workflow to allocate the production rate between the fields. The changes in the optimum allocation decision can be seen on the 0.98 and 0.99 correlation factors. 
Table 6.22: Optimization Sensitivity Analysis of the XLight-Heavy Price Correlation Factor

\begin{tabular}{|c|c|c|c|c|c|c|c|c|}
\hline \multicolumn{9}{|c|}{ Production Rate } \\
\hline \multirow{2}{*}{ XLight-Heavy Price Correlation Factor } & \multicolumn{4}{|c|}{ Xlight } & \multicolumn{4}{|c|}{ Medium } \\
\hline & Field 4 & Field 5 & Field 6 & Field 7 & Field 16 & Field 17 & Field 18 & Field 19 \\
\hline 0.1 & 247 & 164 & 82 & 113 & 123 & 871 & 822 & 822 \\
\hline 0.8 & 247 & 164 & 82 & 113 & 123 & 871 & 822 & 822 \\
\hline 0.98 & 822 & 164 & 274 & 113 & 123 & 308 & 617 & 822 \\
\hline 0.99 & 822 & 548 & 274 & 377 & 123 & 308 & 247 & 545 \\
\hline
\end{tabular}

Table 6.23 shows the optimum allocation decisions as the XXLight-Heavy Price Correlation Factor varies. The correlation factor is the slope in the regression correlation equation presented in the price correlation section. The table shows only the fields that are impacted by changing the correlation factor. The highlighted correlation factors are those where a change in the optimum allocation decision occurs. As the XXLight-Heavy correlation factor decreases, reducing the value of the XXLight crude, the production rates allocated to the XXLight crude fields also decreases. The optimizer uses a workflow to allocate the production rate between the fields. The changes in the optimum allocation decision can be seen on the 0.95 and 0.96 correlation factors. Due to the fact that the total potential of the XXlight crude fields is relatively small, a production reduction from only two Medium crude fields (Field 18 and 19) are enough to increase all the XXlight fields to maximum potential. Table 6.24 shows the optimum allocation decision by crude grades.

Table 6.23: Optimization Sensitivity Analysis of the XXLight-Heavy Price Correlation

\begin{tabular}{|c|c|c|c|c|c|c|c|}
\hline \multicolumn{8}{|c|}{ Production Rate } \\
\hline \multirow{2}{*}{ XXLight-Heavy Price Correlation Factor } & \multicolumn{3}{|c|}{ XXLight } & \multicolumn{4}{|c|}{ Medium } \\
\hline & Field 1 & Field 2 & Field 3 & Field 16 & Field 17 & Field 18 & Field 19 \\
\hline 0.10 & 46 & 99 & 62 & 123 & 308 & 451 & 822 \\
\hline 0.94 & 46 & 99 & 62 & 123 & 308 & 451 & 822 \\
\hline 0.95 & 46 & 159 & 205 & 123 & 308 & 247 & 822 \\
\hline 0.96 & 153 & 329 & 205 & 123 & 308 & 247 & 545 \\
\hline
\end{tabular}




\subsubsection{The GeOMetric Brownian Motion (GBM)}

The GBM is a widely used stochastic price forecasting model due to its simplicity and practicality. In this section, the optimum allocation decision of the GBM model is presented and analyzed. The GBM formulation is discussed in the section on Price Formulation.

\subsubsection{The Optimum Decision of the Geometric Brownian Motion Forecast Model}

Table 6.23 and Figure 6.15 show the optimum allocation decision of the GBM model with a mean of the total NPV MM\$3,272,395 and a standard deviation of MM\$ 4,167,279. The GBM model leads to the highest mean of total NPV and standard deviation among all the price forecasting models. These large values resulted from the diffusion process of log-normal distribution (GBM model). The large expected value of the total NPV means that this large number is the return of the project (on average), and the large standard deviation reveals the tremendous uncertainty involved in the GBM price modeling. This uncertainty can be seen in Figure 6.16 where the long-flat tail shows the possibility of "rare events" (very high crude prices). The optimum allocation is similar to that of the Floating Price model. The GBM is a diffused log-normal distribution with a drift term; the drift term imposes the Floating Price effect on the GBM model. Note that the mean of the total NPV of the GBM model is close to the total NPV of the Floating Price model. The drift term in the GBM and the rate of price increases in the Floating Price model might be an explanation for their similar optimum allocation decisions. The difference in the optimum allocation decision between the GBM and the Flat Price models is negligible as can be seen in Figure 6.23. Figure 6.24 shows the optimum allocation by crude grades. 
Table 6.23: The GMB Optimum Allocation Decision

\begin{tabular}{|c|c|c|c|c|}
\hline Field & $\begin{array}{l}\text { Optimum } \\
\text { Production Rate } \\
\text { (MBD) }\end{array}$ & $\begin{array}{l}\text { Production } \\
\text { Rate Status }\end{array}$ & $\begin{array}{l}\text { "Flat Price" } \\
\text { Optimum } \\
\text { Production } \\
\text { Rate } \\
\end{array}$ & $\begin{array}{c}\text { Difference } \\
\text { Between } \\
\text { Current and } \\
\text { Flat Price Case }\end{array}$ \\
\hline 1 & 153 & Upper Bound & 153 & 0 \\
\hline 2 & 329 & Upper Bound & 329 & 0 \\
\hline 3 & 205 & Upper Bound & 205 & 0 \\
\hline 4 & 822 & Upper Bound & 822 & 0 \\
\hline 5 & 548 & Upper Bound & 548 & 0 \\
\hline 6 & 274 & Upper Bound & 274 & 0 \\
\hline 7 & 377 & Upper Bound & 377 & 0 \\
\hline 8 & 110 & Upper Bound & 33 & 77 \\
\hline 9 & 247 & Upper Bound & 247 & 0 \\
\hline 10 & 342 & Upper Bound & 342 & 0 \\
\hline 11 & 548 & Upper Bound & 548 & 0 \\
\hline 12 & 822 & Upper Bound & 822 & 0 \\
\hline 13 & 438 & Upper Bound & 438 & 0 \\
\hline 14 & 438 & Upper Bound & 438 & 0 \\
\hline 15 & 411 & Upper Bound & 411 & 0 \\
\hline 16 & 123 & Lower_Bound & 123 & 0 \\
\hline 17 & 308 & Lower_Bound & 308 & 0 \\
\hline 18 & 247 & Lower_Bound & 247 & 0 \\
\hline 19 & 468 & & 545 & -77 \\
\hline 20 & 205 & Lower_Bound & 205 & 0 \\
\hline 21 & 222 & Lower_Bound & 222 & 0 \\
\hline 22 & 197 & Lower_Bound & 197 & 0 \\
\hline 23 & 164 & Lower_Bound & 164 & 0 \\
\hline
\end{tabular}

Table 6.24: The GBM Optimum Allocation Decision by Crude Grade

\begin{tabular}{|lc|}
\hline Crude Grade & $\begin{array}{c}\text { Optimal Production } \\
\text { Rate (MBD) }\end{array}$ \\
\hline XXL & 688 \\
\hline XI & 2,021 \\
AL & 3,356 \\
AM & 1,147 \\
AH & 789 \\
sum & 8,000 \\
\hline
\end{tabular}




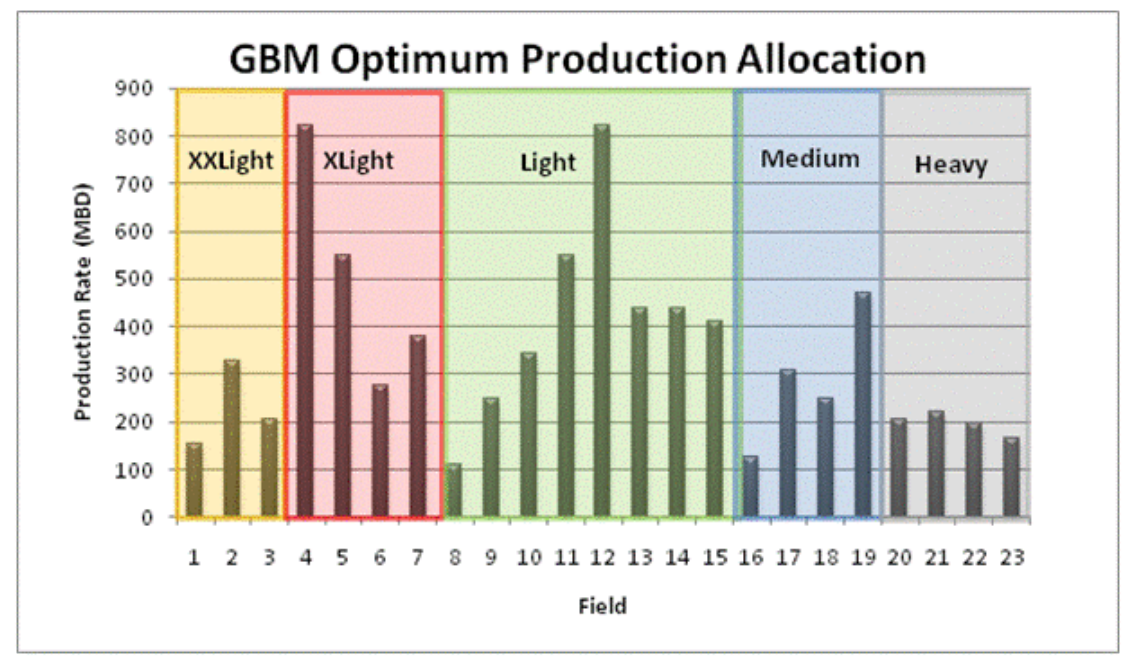

Figure 6.15: The Optimum Allocation Decision the GBM

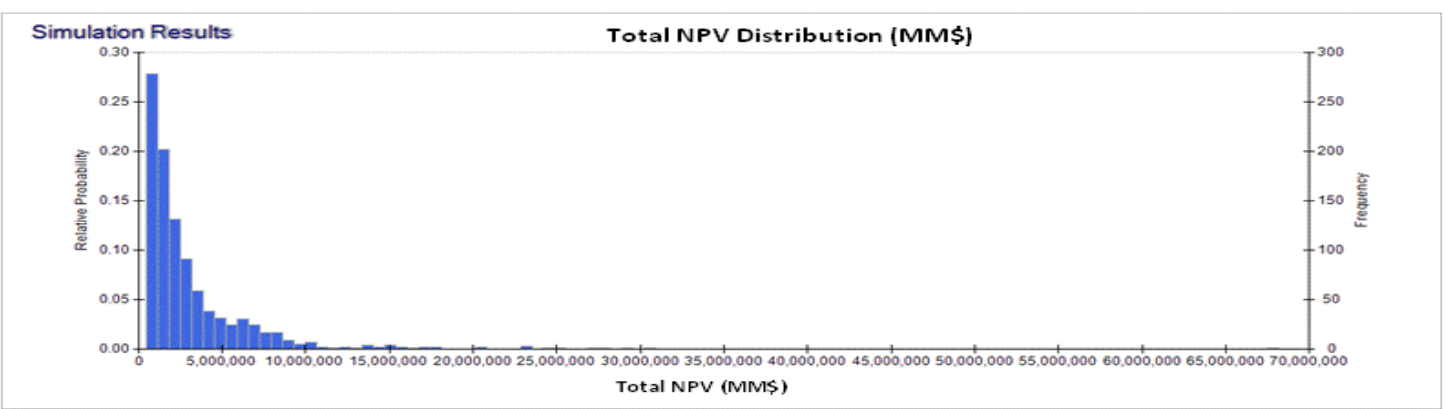

Figure 6.16: Total NPV Distribution of the GBM Model

\subsubsection{The Sensitivity Analysis of the Geometric Brownian Motion (GBM)}

This section presents a sensitivity analysis of the total NVP and the optimum allocation decisions by varying the modeling parameters of the GBM model. The tornado chart of the total NPV is generated by varying the modeling parameters (e.g., price drift and volatility, initial price, etc.) by $\pm 10 \%$ as shown in Figure 6.17. Each parameter is varied and analyzed individually. The most significant parameters, for NPV calculations, are the Heavy crude price drift and the Heavy crude price volatility as shown in the figure below. Note that the two parameters are more significant than the discount rate and price correlation factors, which are the most significant parameters in the Flat Price, Floating 
Price, and ILD models. Although the modeling parameters are significant for the total NPV calculations, they do not impact the optimum allocation decision as discussed below.

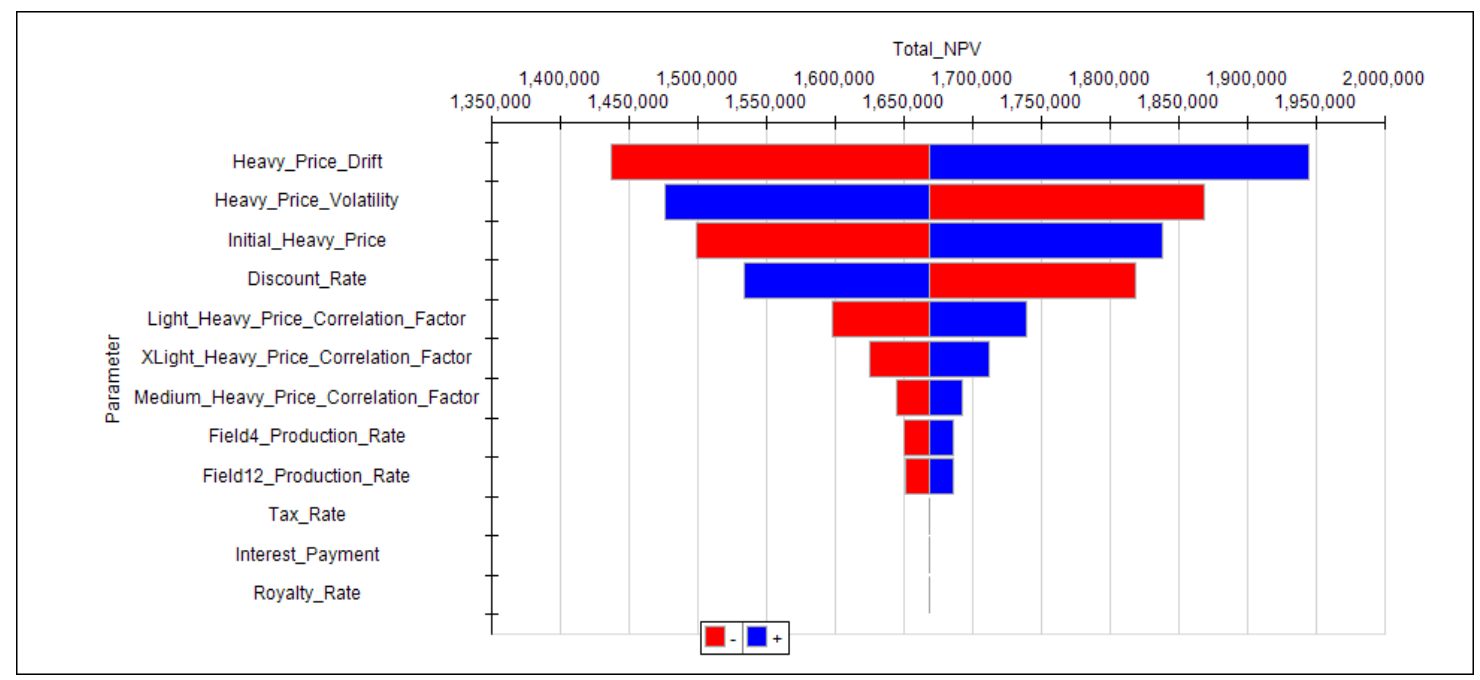

Figure 6.17: Total NPV Tornado Chart by Varying Parameters $\pm 10 \%$

Figure 6.18 is a sensitivity analysis of the mean of total NPV by varying the drift of the Heavy crude price; the steep curve is expected since the drift is the most significant parameters in the tornado chart. The curve implies that as the drift increases, its impact increases as well. Figures 6.19 and 6.20, a 50-iteration GBM model, reveals the impact of the drift on the price forecast model, which impacts the total NPV. based on the two figures, the GBM with "0" drift, prices does not exceed \$ 400 per barrel while prices reach $\$ 9,000$ per barrel with " 0.3 " drift. In other words, the small change in the price drift caused a huge in change in the price forecast model and hence the total NPV. On the other hand, the drift does not impact the optimum allocation decision; analysis was conducted to investigate the impact of the parameters on the optimum allocation decision. 


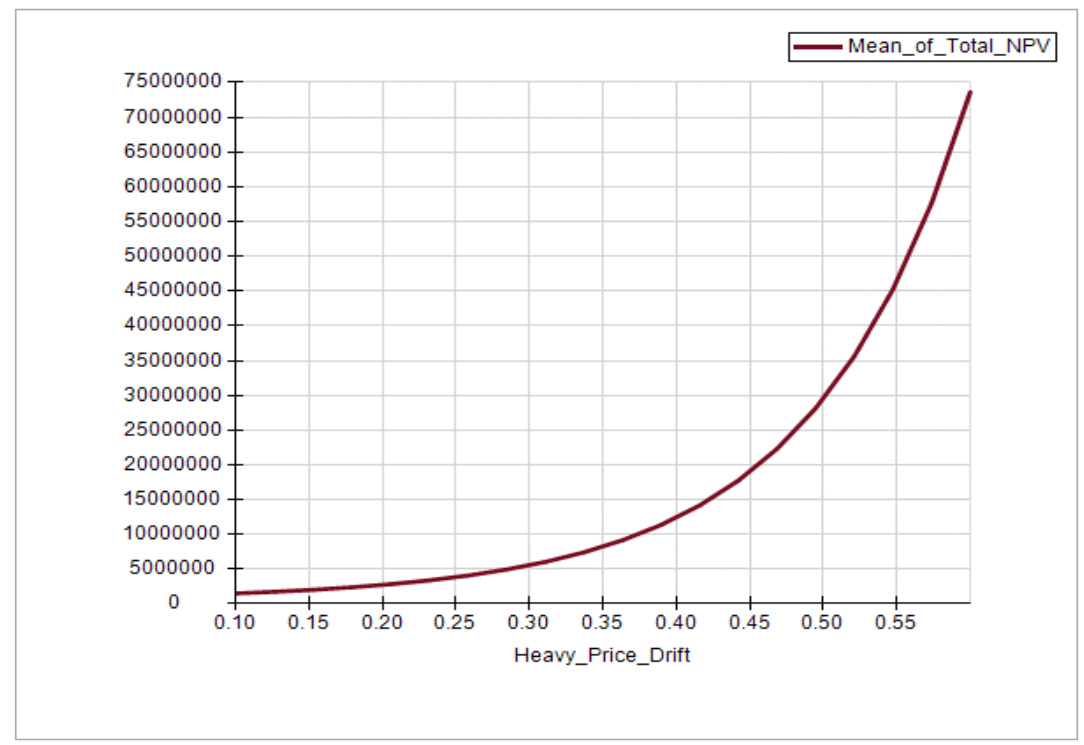

Figure 6.18: Sensitivity Analysis of the Mean of Total NPV to the Drift of the Heavy Crude Price

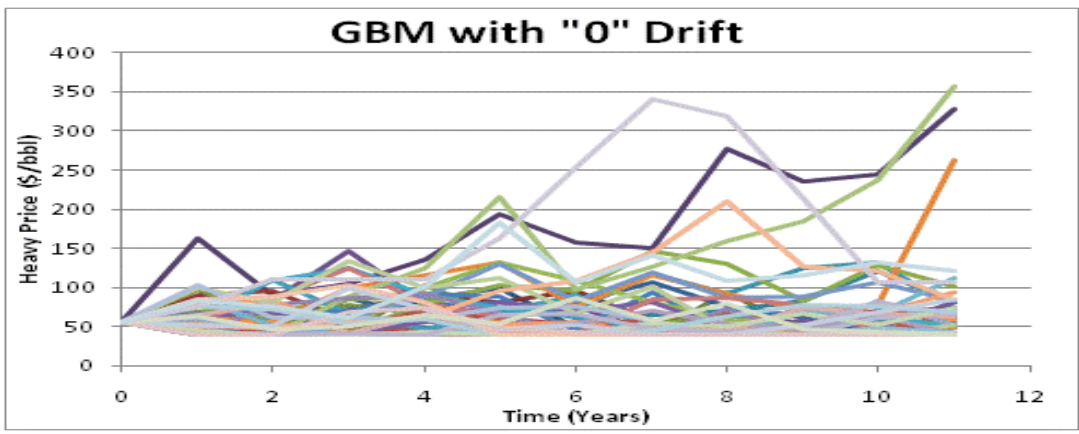

Figure 6.19: The GBM Price Model with “0” Price Drift (50 Iterations)

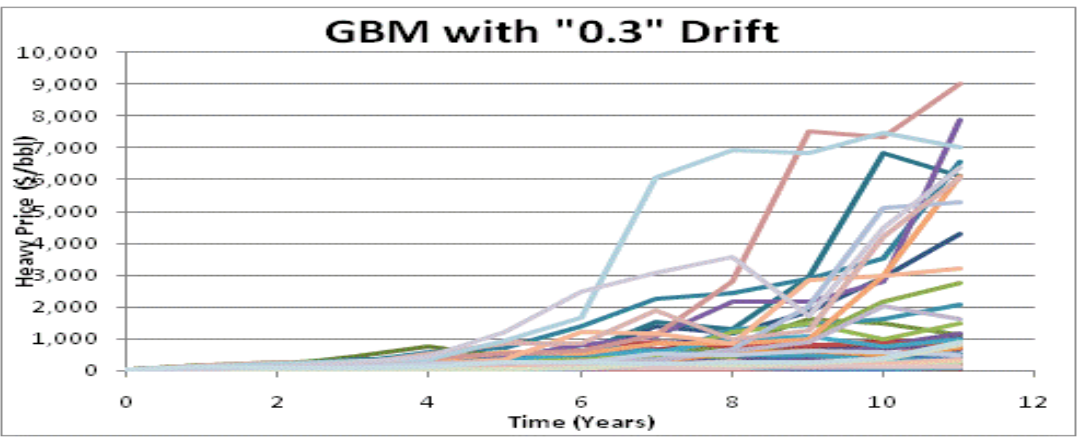

Figure 6.20: The GBM Price Model with “0.3” Price Drift (50 Iterations) 
Figure 6.21 is a sensitivity analysis of the mean of total NPV by varying the volatility of the Heavy crude price. Note that the mean of the total NPV slightly decreases as volatility goes from 0.1 to 0.23 ; then the mean increases sharply. A possible explanation for this behavior is that as volatility increases, more and more prices hit the minimum $\$ 40$ reducing the mean of the total NPV (Figure 6.22); when volatility reaches 0.25 , the price forecasts increase to abnormally high prices that drastically increase the mean of the total NPV (Figure 6.23). Figures 6.22 and 6.23, 50-iterations GBM models, reveal the impact of volatility on the price model, which impacts the mean of the total NPV. At a volatility of 0.2 in the GBM model, prices reach $\$ 2,000$ per barrel while they reach $\$ 5,000$ per barrel at 0.3 volatility. Note that only a 0.1 change in price volatility can cause a drastic impact on the prices and the total NPV. On the other hand, the Heavy price volatility does not impact the optimum allocation decision. The impact of the modeling parameters on the optimum allocation decision is analyzed; the result of the analysis shows that the optimum allocation decisions are the same.

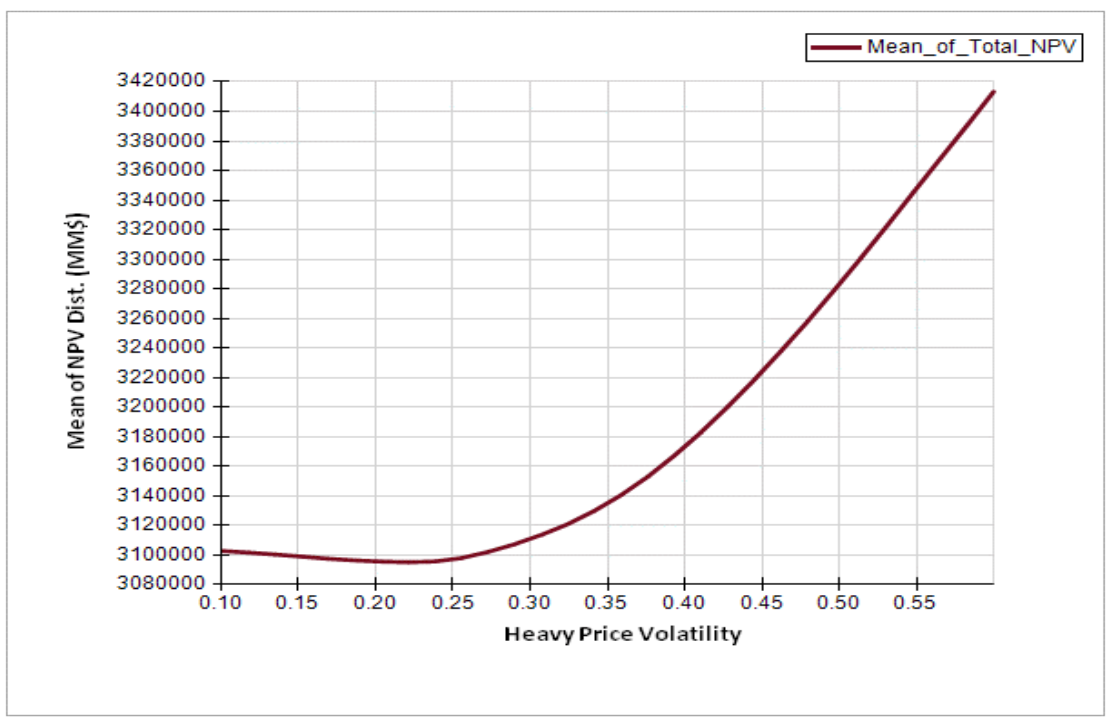

Figure 6.21: Sensitivity Analysis of the Mean of Total NPV to the Volatility of the Heavy Crude Price 


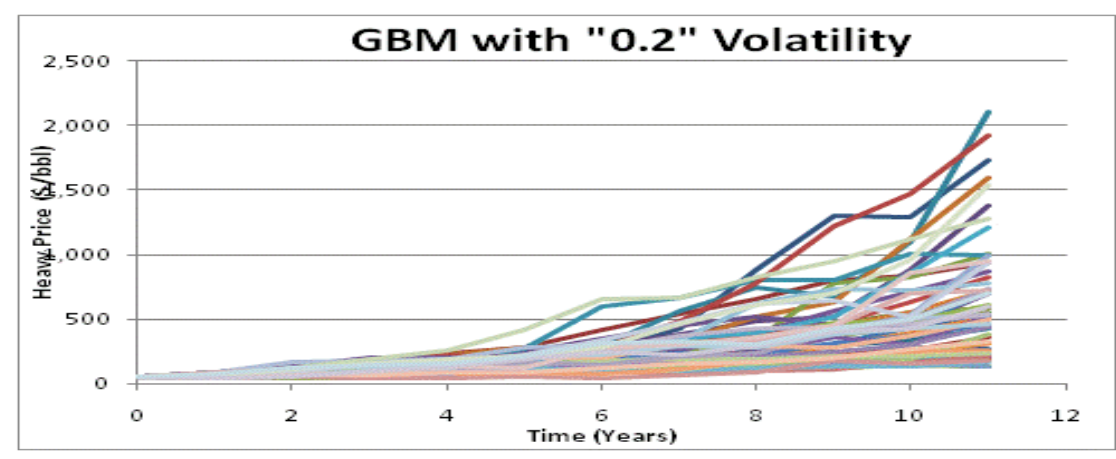

Figure 6.22: The GBM Price Model with “0.2” Price Volatility (50 Iterations)

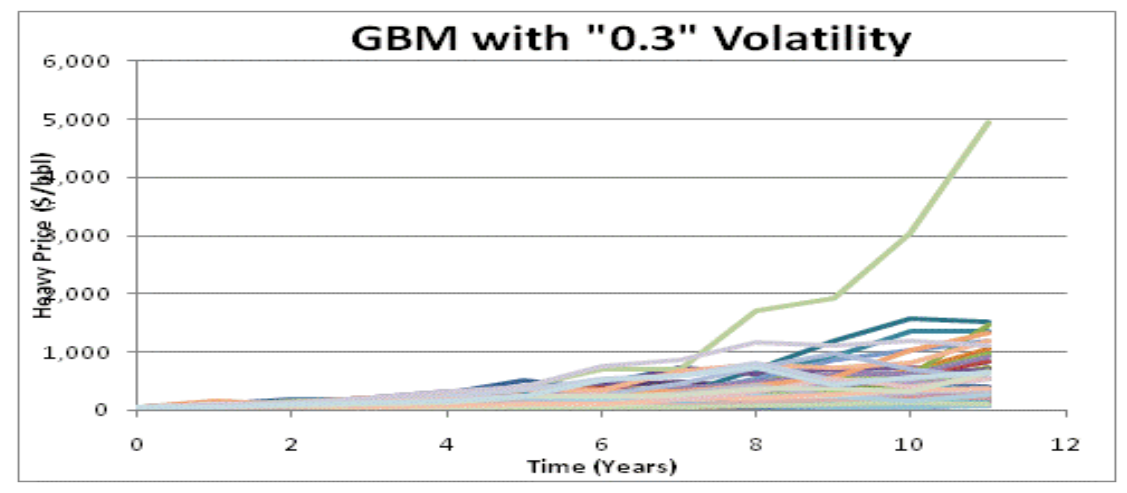

Figure 6.23: The GBM Price Model with “0.3” Price Volatility (50 Iterations)

\subsubsection{The MEAN ReVERSION MOdel (MRM)}

The MRM is the most economically logical price forecast model because it allows for supply to respond to changes in price. In other words, the model mimics the suppliers/ producers response to price changes; in practice, suppliers increase production in response to higher commodity prices to increase profit. This behavior of suppliers tends to lower the prices because of the excess un-sold products. Similarly, the MRM pulls the prices toward the long-term mean when prices increase or decrease. The MRM formulation is discussed in the section on Price Formulation. 


\subsubsection{The Optimum Decision of the Mean Reversion Model (MRM)}

Table 6.25 and Figure 6.24 show the optimum allocation decision of the MRM price forecast model with a mean of total NPV MM\$ 730,535 and a standard deviation of MM\$ 94,248. The mean of the total NPV is close to total NPV of the Flat Price model. The large standard deviation reveals the uncertainty involved in the MRM price modeling. This uncertainty can be seen in the total NPV probability density function (Figure 6.25); the total NPV is log-normally distributed because of the hedging process at the minimum Heavy crude price of $\$ 40$. In other words, the minimum $\$ 40$ reduced the size of the left hand side tail. The optimum allocation is similar to the Flat Price optimum decision. Figure 6.26 shows the optimum allocation decision by crude grades.

Table 6.25: The MRM Optimum Allocation Decision

\begin{tabular}{|ccccc|}
\hline Field & $\begin{array}{c}\text { Optimum } \\
\text { Production Rate } \\
\text { (MBD) }\end{array}$ & $\begin{array}{c}\text { Production } \\
\text { Rate Status }\end{array}$ & $\begin{array}{c}\text { "Flat Price" } \\
\text { Optimum } \\
\text { Production } \\
\text { Rate }\end{array}$ & $\begin{array}{c}\text { Difference } \\
\text { Between } \\
\text { Current and } \\
\text { Flat Price Case }\end{array}$ \\
\hline 1 & 153 & Upper Bound & 153 & 0 \\
2 & 329 & Upper Bound & 329 & 0 \\
3 & 205 & Upper Bound & 205 & 0 \\
4 & 822 & Upper Bound & 822 & 0 \\
5 & 548 & Upper Bound & 548 & 0 \\
6 & 274 & Upper Bound & 274 & 0 \\
7 & 377 & Upper Bound & 377 & 0 \\
8 & 33 & Lower_Bound & 33 & 0 \\
9 & 247 & Upper Bound & 247 & 0 \\
10 & 342 & Upper Bound & 342 & 0 \\
11 & 548 & Upper Bound & 548 & 0 \\
12 & 822 & Upper Bound & 822 & 0 \\
13 & 438 & Upper Bound & 438 & 0 \\
14 & 438 & Upper Bound & 438 & 0 \\
15 & 411 & Upper Bound & 411 & 0 \\
16 & 123 & Lower_Bound & 123 & 0 \\
17 & 308 & Lower_Bound & 308 & 0 \\
18 & 247 & Lower_Bound & 247 & 0 \\
19 & 545 & Lower_Bound & 205 & 0 \\
20 & 205 & Lower_Bound & 222 & 0 \\
21 & 222 & Lower_Bound & 197 & 0 \\
22 & 197 & Lower_Bound & 164 & 0 \\
23 & 164 & & & 0 \\
\hline
\end{tabular}


Table 6.26: The MRM Optimum Allocation Decision by Crude Grade

\begin{tabular}{|lc|}
\hline \multicolumn{1}{|c|}{ Optimal Production } \\
Crude Type & Rate (MBD) \\
XXL & 688 \\
\hline $\mathrm{XI}$ & 2,021 \\
$\mathrm{AL}$ & 3,279 \\
$\mathrm{AM}$ & 1,223 \\
$\mathrm{AH}$ & 789 \\
sum & 8,000 \\
\hline
\end{tabular}

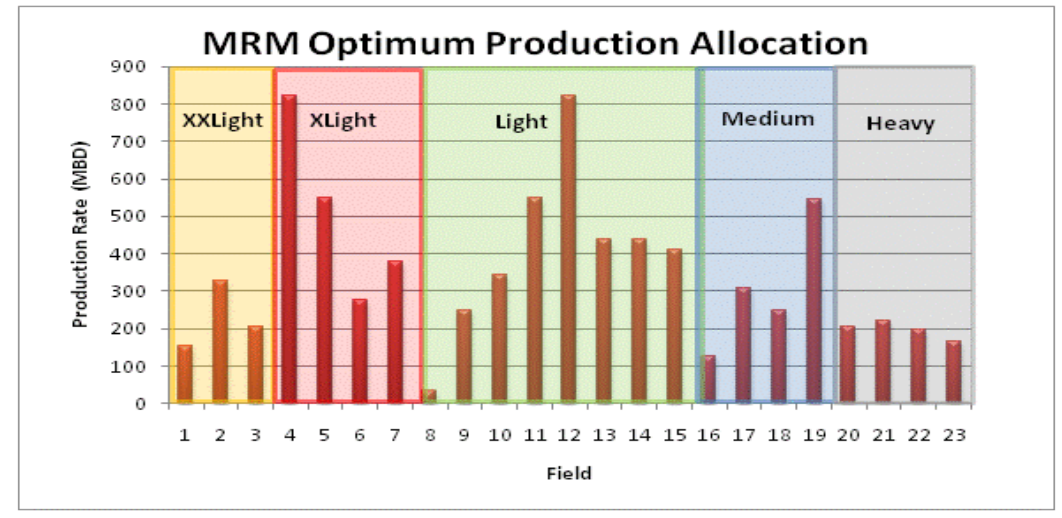

Figure 6.24: The MRM Optimum Allocation Decision

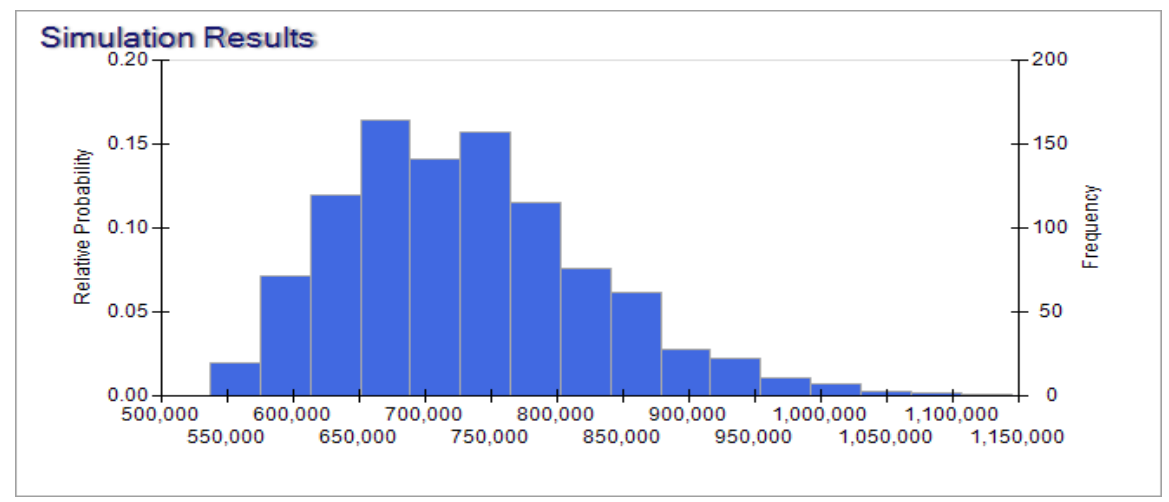

Figure 6.25: Total NPV Distribution of the MRM Price Forecast Model 


\subsubsection{The Sensitivity Analysis of the Mean Reversion Model (MRM)}

This section presents an analysis of the impact of the parameters on the total NVP and the optimum allocation decision. The tornado chart of the total NPV is generated by varying the modeling parameters (e.g., long-run mean price, price volatility...etc.) by $\pm 10 \%$ as shown in Figure 6.26. Each parameter is varied and analyzed individually. As the figure shows, the MRM modeling parameter, Long-term Equilibrium Price, impacts the total NPV the most. Price volatility is another significant parameter shown in the figure. On the other hand, the impact of the price reversion speed is not as significant as of the long-term mean price; thus, the price reversion speed is not listed in the tornado chart. Although the modeling parameters impact the total NPV significantly, they do not impact the optimum allocation decision as discussed below.

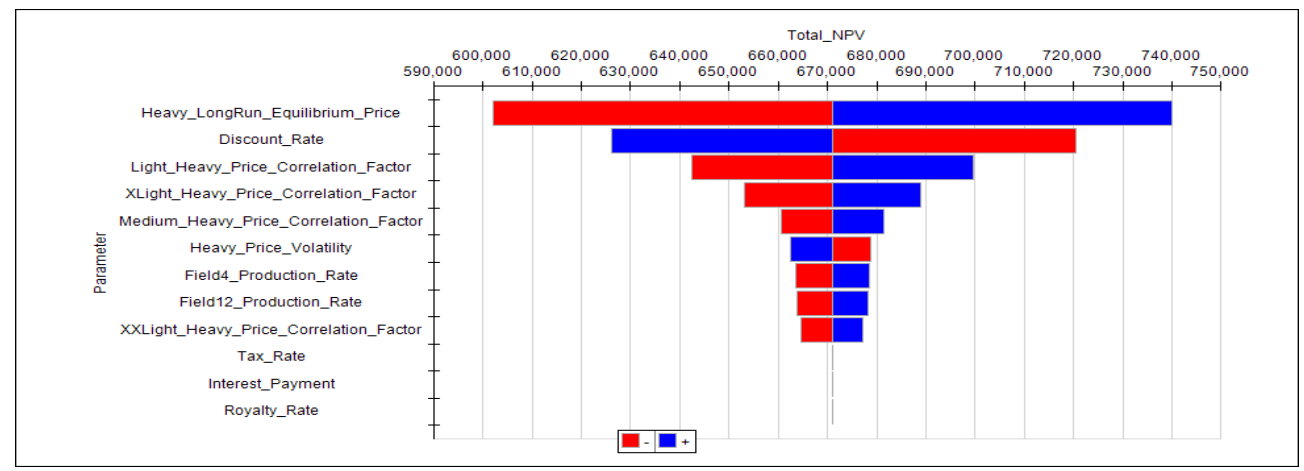

Figure 6.26: Total NPV Tornado Chart by Varying Parameters $\pm 10 \%$

Figure 6.27 shows a sensitivity analysis of the Heavy crude price reversion speed; as reversion speed increases, its impact on the total NPV decreases. The reversion speed is how fast the price reverts to the long-run mean price with time. In other words, as the reversion speed goes up, the random prices revert very quickly to the long-run equilibrium price (Figure 6.28). On the other hand, if the reversion speed is slow, random 
prices take longer to revert to the equilibrium price (prices revert at a low speed) as shown in Figure 6.29.

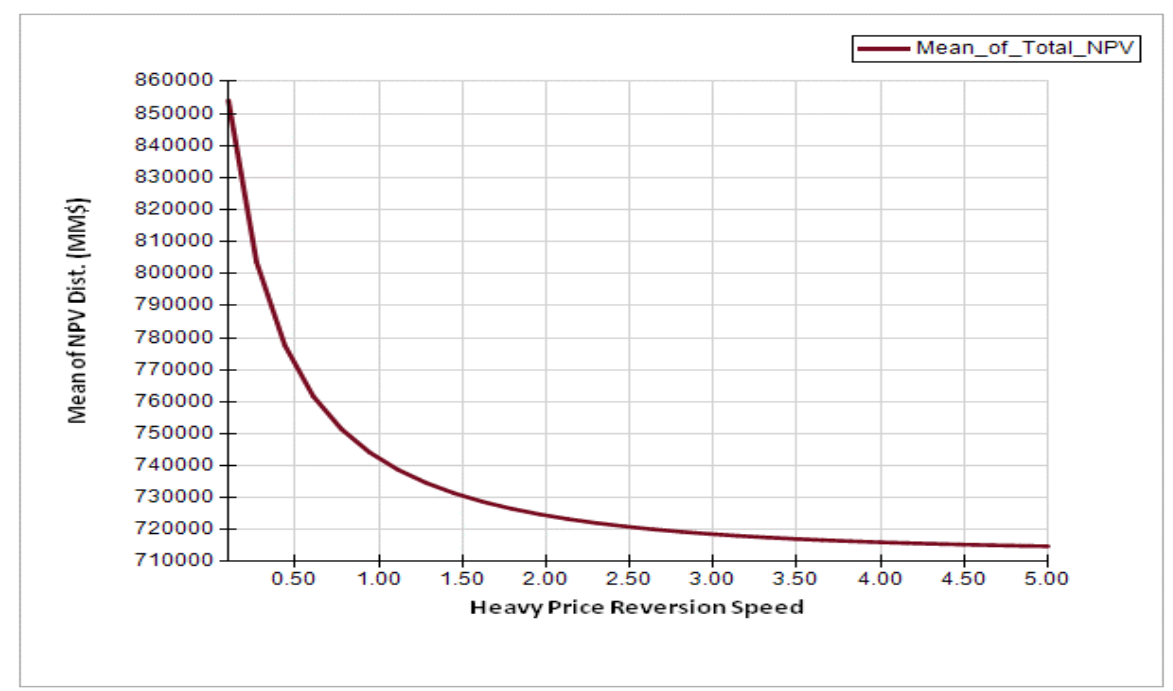

Figure 6.27: Sensitivity Analysis of the Mean of Total NPV to the Reversion Speed of the Heavy Price

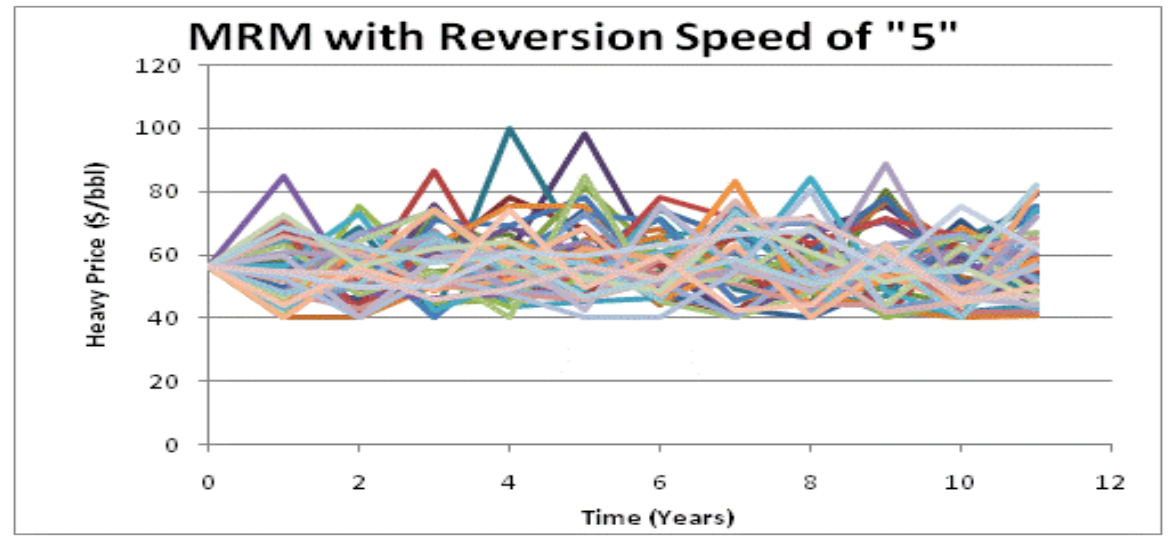

Figure 6.28: The MRM with 5 Reversion Speed 


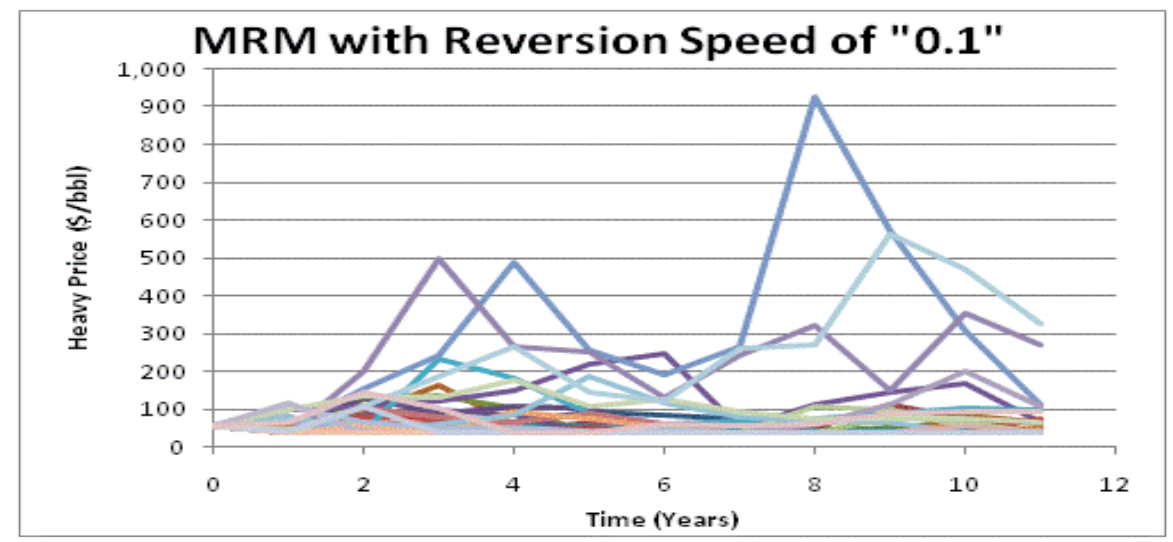

Figure 6.29: The MRM with "0.1” Reversion Speed

Figures 6.30 and 6.31 confirm the high sensitivity of the mean of total NPV to the Heavy crude long-run mean price and the Heavy price volatility, respectively. However, the two parameters do not impact the optimum allocation decision.

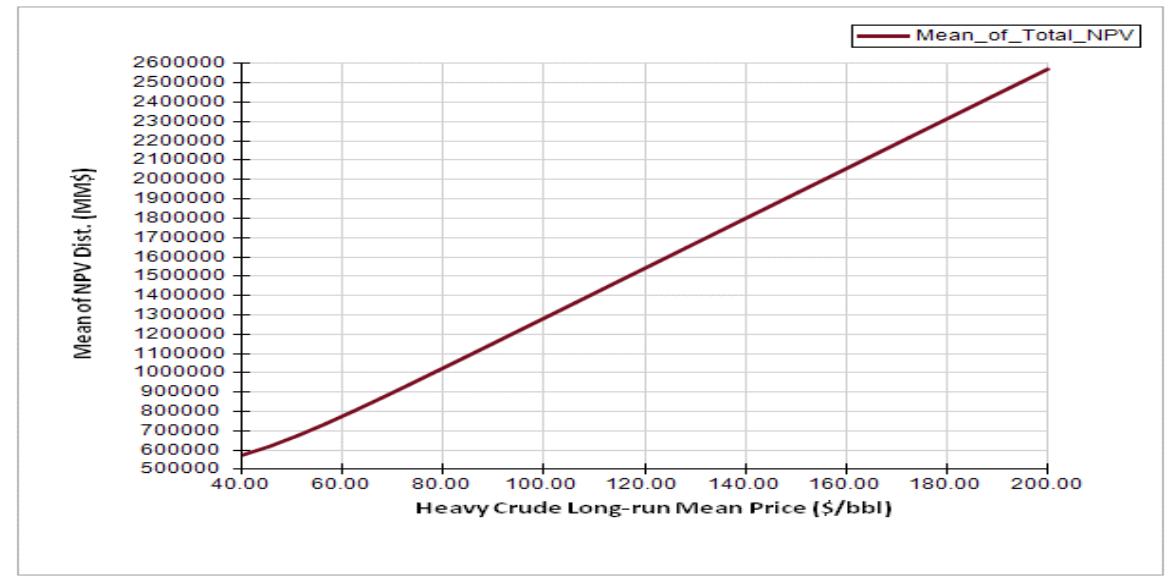

Figure 6.30: Sensitivity Analysis of the Mean of Total NPV to the Long-run Mean price of the Heavy Crude 


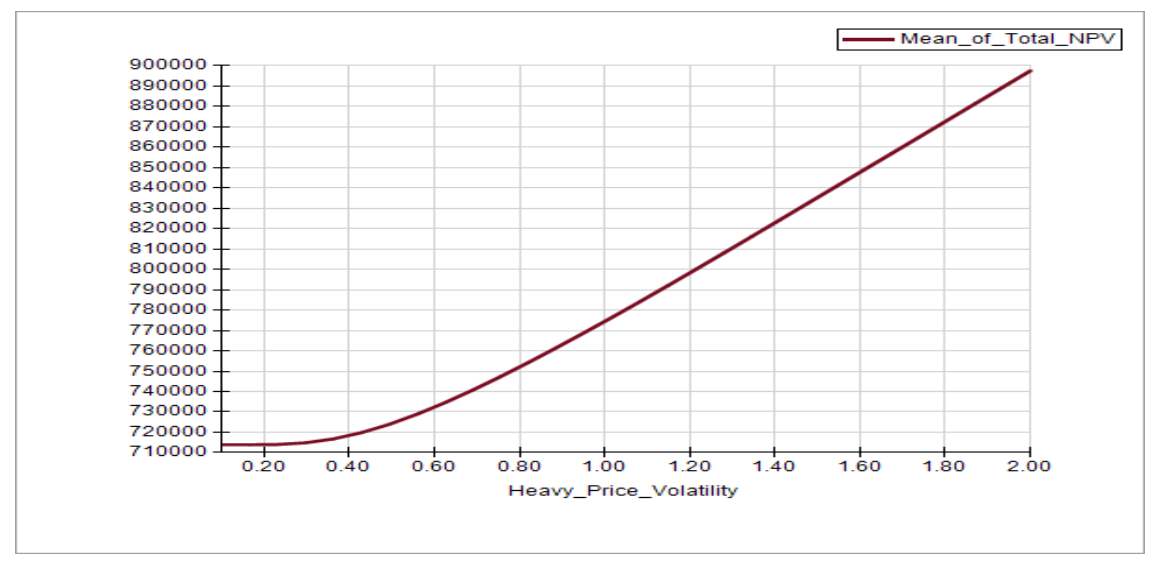

Figure 6.31: Sensitivity Analysis of the Mean of Total NPV to the Volatility of the Heavy Price

\subsubsection{THE MEAN REVERSION WITH JUMPS MODEL (MRJM)}

The Mean Reversion with Jumps model is a combination of the Mean Reversion model and Poisson distribution. The model relates information arrival to price changes. The mean reversion process accounts for the normal news arrival, while the jumps accounts for the abnormal information arrival (e.g., war, natural disaster, etc.). The formulation process is discussed in the section on Price Formulation.

\subsubsection{The Optimum Decision of the Mean Reversion with Jumps Model (MRJM)}

Table 6.27 and Figure 6.32 show the optimum allocation decision of the MRJM forecast model with a mean of total NPV MM\$ 793,287 and a standard deviation of MM\$ 598,866. The large standard deviation reveals the huge price uncertainty, which can be seen in Figure 6.33. The long flat tale in Figure 6.33 accounts for the jumps (rare events) in the price model. Note that the mean of the total NPV for the MRJM is close to that of the MRM; however, the standard deviations are far different because of the jumps effects. Figure 6.28 shows the optimum allocation decision by crude grades. 
Table 6.27: MRJM Optimum Allocation Decision

\begin{tabular}{|c|c|c|c|c|}
\hline Field & $\begin{array}{l}\text { Optimum } \\
\text { Production Rate } \\
\text { (MBD) }\end{array}$ & $\begin{array}{l}\text { Production } \\
\text { Rate Status }\end{array}$ & $\begin{array}{l}\text { "Flat Price" } \\
\text { Optimum } \\
\text { Production } \\
\text { Rate } \\
\end{array}$ & $\begin{array}{c}\text { Difference } \\
\text { Between } \\
\text { Current and } \\
\text { Flat Price Case }\end{array}$ \\
\hline 1 & 153 & Upper Bound & 153 & 0 \\
\hline 2 & 329 & Upper Bound & 329 & 0 \\
\hline 3 & 205 & Upper Bound & 205 & 0 \\
\hline 4 & 822 & Upper Bound & 822 & 0 \\
\hline 5 & 548 & Upper Bound & 548 & 0 \\
\hline 6 & 274 & Upper Bound & 274 & 0 \\
\hline 7 & 377 & Upper Bound & 377 & 0 \\
\hline 8 & 33 & Lower_Bound & 33 & 0 \\
\hline 9 & 247 & Upper Bound & 247 & 0 \\
\hline 10 & 342 & Upper Bound & 342 & 0 \\
\hline 11 & 548 & Upper Bound & 548 & 0 \\
\hline 12 & 822 & Upper Bound & 822 & 0 \\
\hline 13 & 438 & Upper Bound & 438 & 0 \\
\hline 14 & 438 & Upper Bound & 438 & 0 \\
\hline 15 & 411 & Upper Bound & 411 & 0 \\
\hline 16 & 123 & Lower_Bound & 123 & 0 \\
\hline 17 & 308 & Lower_Bound & 308 & 0 \\
\hline 18 & 247 & Lower_Bound & 247 & 0 \\
\hline 19 & 545 & & 545 & 0 \\
\hline 20 & 205 & Lower_Bound & 205 & 0 \\
\hline 21 & 222 & Lower_Bound & 222 & 0 \\
\hline 22 & 197 & Lower_Bound & 197 & 0 \\
\hline 23 & 164 & Lower_Bound & 164 & 0 \\
\hline
\end{tabular}

Table 6.28: MRJM Optimum Allocation Decision by Crude Grade

\begin{tabular}{|lc|}
\hline Crude Type & $\begin{array}{c}\text { Optimal Production } \\
\text { Rate (MBD) }\end{array}$ \\
\hline$X X L$ & 688 \\
XI & 2,021 \\
\hline AL & 3,279 \\
AM & 1,223 \\
AH & 789 \\
sum & 8,000 \\
\hline
\end{tabular}




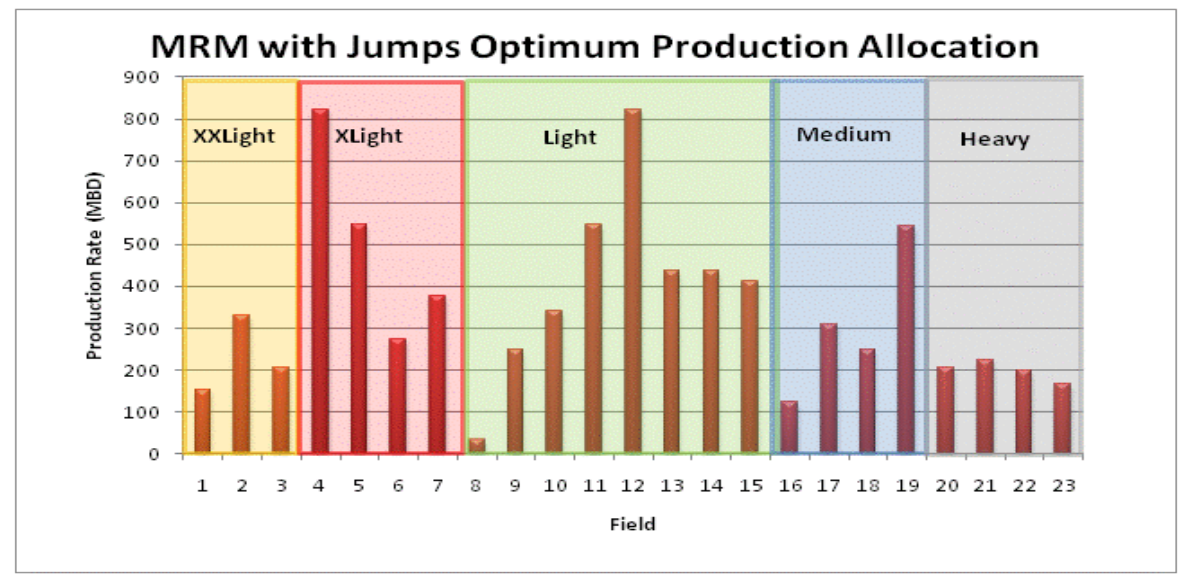

Figure 6.32: MRJM Optimum Allocation Decision

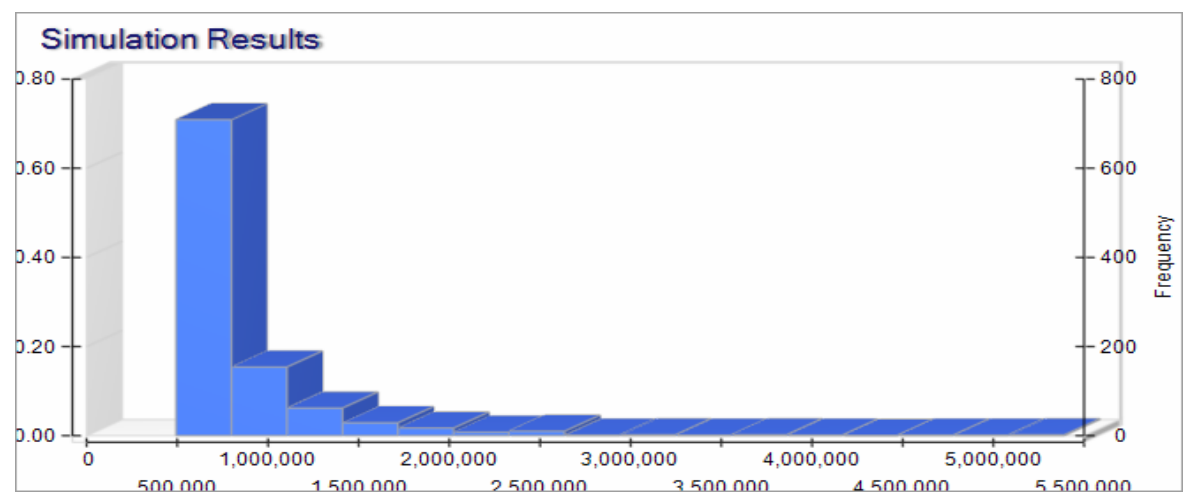

Figure 6.33: MRJM Total NPV Probability Density Function

\subsubsection{The Sensitivity Analysis of the Mean Reversion with Jumps Model (MRJM)}

This section presents an analysis of the impact of the parameters on the total NVP and the optimum allocation decision. The tornado chart of the total NPV is generated by varying the modeling parameters (e.g., long-run mean price, price volatility, etc.) by $\pm 10 \%$ as shown in Figure 6.33 . Each parameter is varied and analyzed individually. As the figure shows, the MRJM modeling parameters, "mean of squared jump size" and "jump frequency", are among the most significant parameters. Although the modeling parameters impact the total NPV significantly, they do not impact the optimum allocation decision as discussed below. 


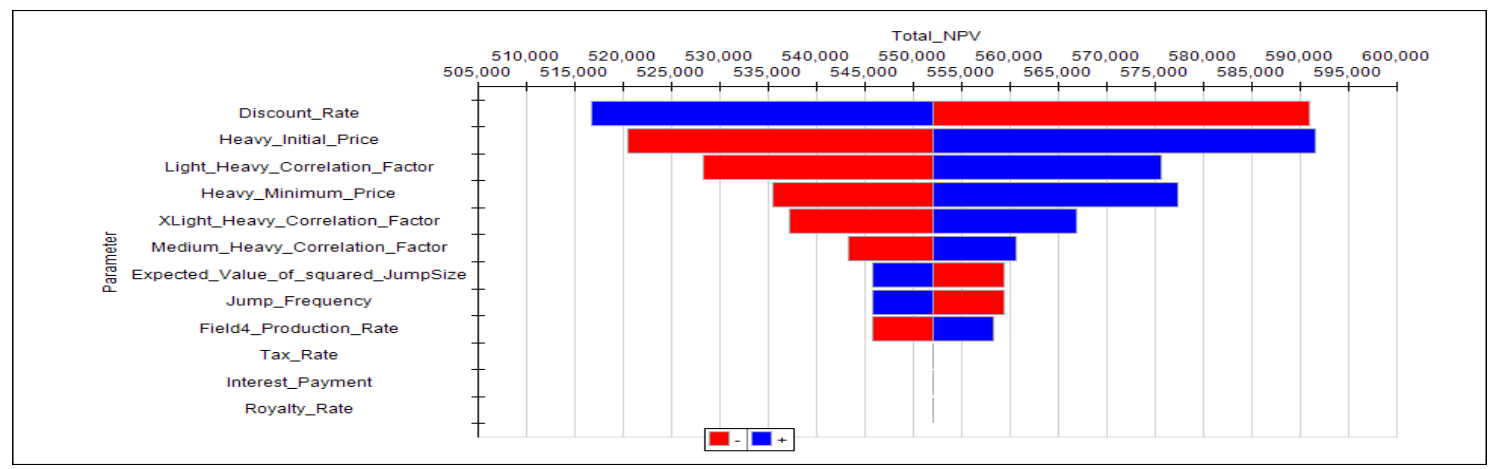

Figure 6.34: Total NPV Tornado Chart by Varying Parameters $\pm 10 \%$

The sensitivity analysis of the price reversion speed is similar to the reversion speed analysis for the MRM model. Figure 6.35 shows the sensitivity of the mean of total NPV to the Heavy long-run mean price. The impact of the long-term mean price is smaller than of the MRM due to the jumps effect. In other words, the jumps decreases the importance of the long-run mean price, so the crude oil price can suddenly be a multiple of the long-run mean price because of the jumps. As the long-run mean price increases, its effect decreases as shown in the decreasing slope of Figure 6.34.

The impact of the Heavy crude price volatility is analyzed; the volatility impact is not predictable in the MRJM because of the jumps. In other words, the jumps effect diminishes the impact of volatility; so, the jumps dictate the volatility of the model; the impact of the jumps volatility is greater than the impact of the price volatility.

Although jumps frequency has a significant impact on the mean of total NPV, this impact is not predictable. The jumps frequency depends on Poisson distribution, which is not predictable. As shown in Figure 6.36, the impact of the jumps frequency can be positive or negative for a specific period of time as the frequency increases; however, the general impact (workflow) is increasing as the frequency increases. 
Figure 6.37 shows the impact of the jumps size on the mean of total NPV. As expected, the impact increases as the jumps size increases; this is clear from the steep curve. Figure 6.38 shows the impact of the mean of the squared jumps size. The flat end of the curve implies that as the mean of the squared jump size increases, its impact decreases.

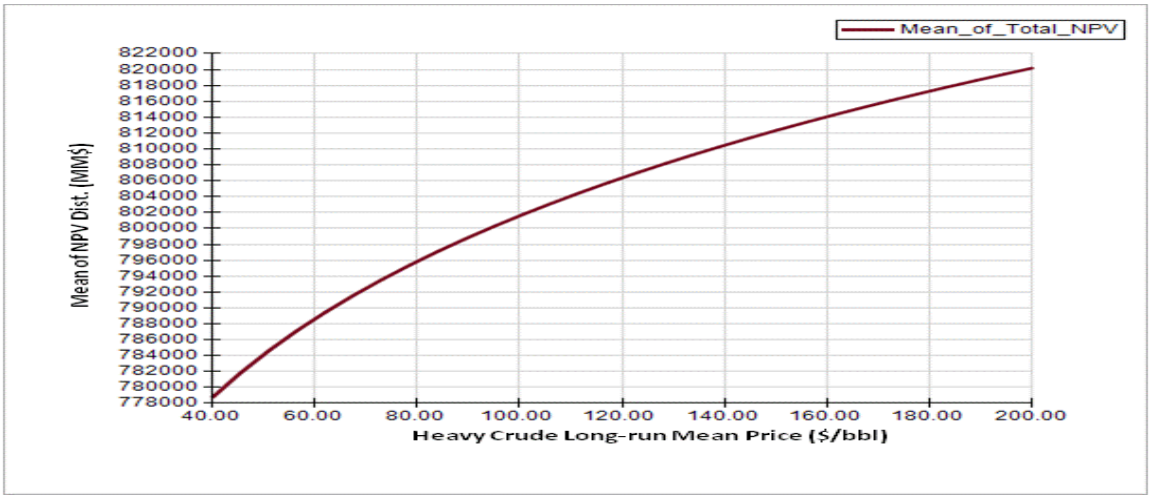

Figure 6.35: Sensitivity Analysis of the Mean of Total NPV to the Long-run Mean Price of the Heavy Crude

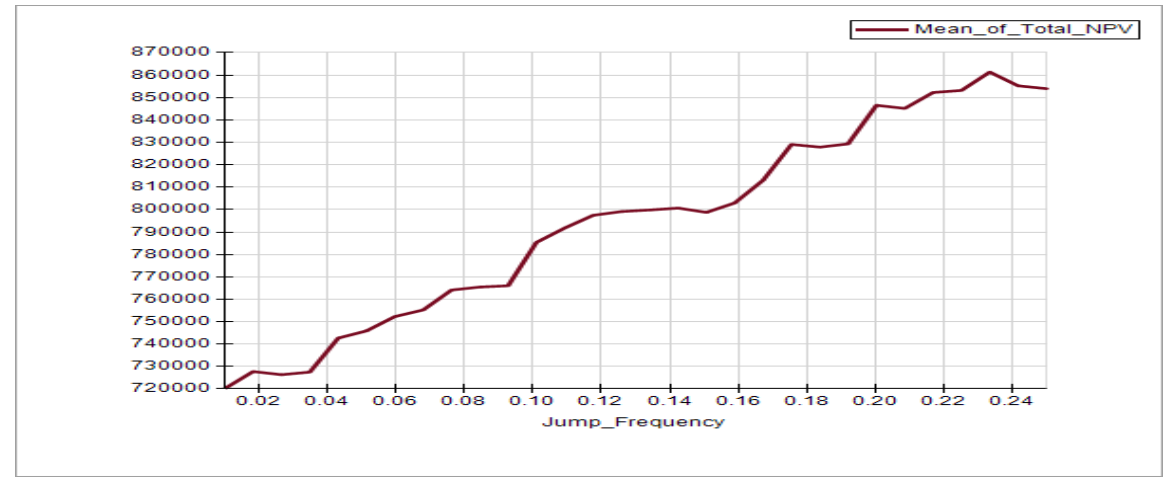

Figure 6.36: Sensitivity of Mean of Total NPV to Jump Frequency 


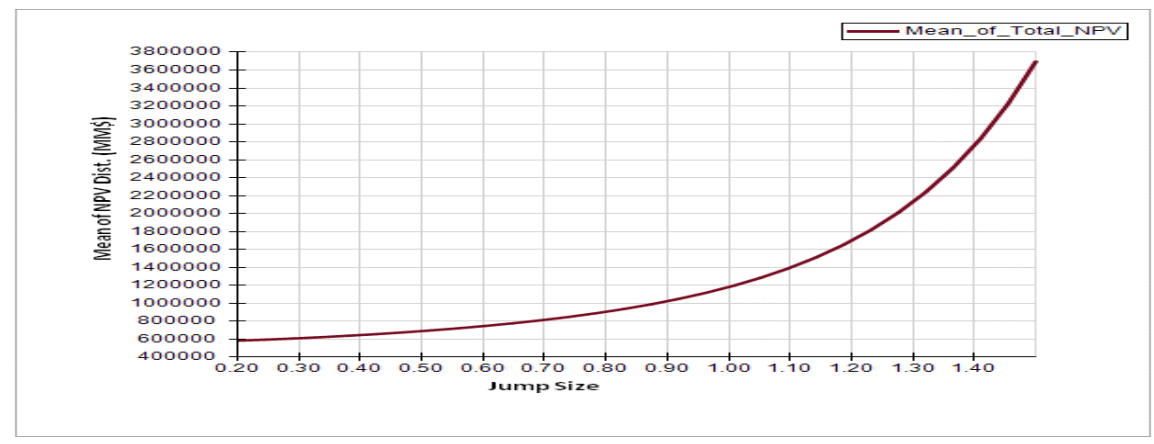

Figure 6.37: Sensitivity of Mean of Total NPV to Jump Size

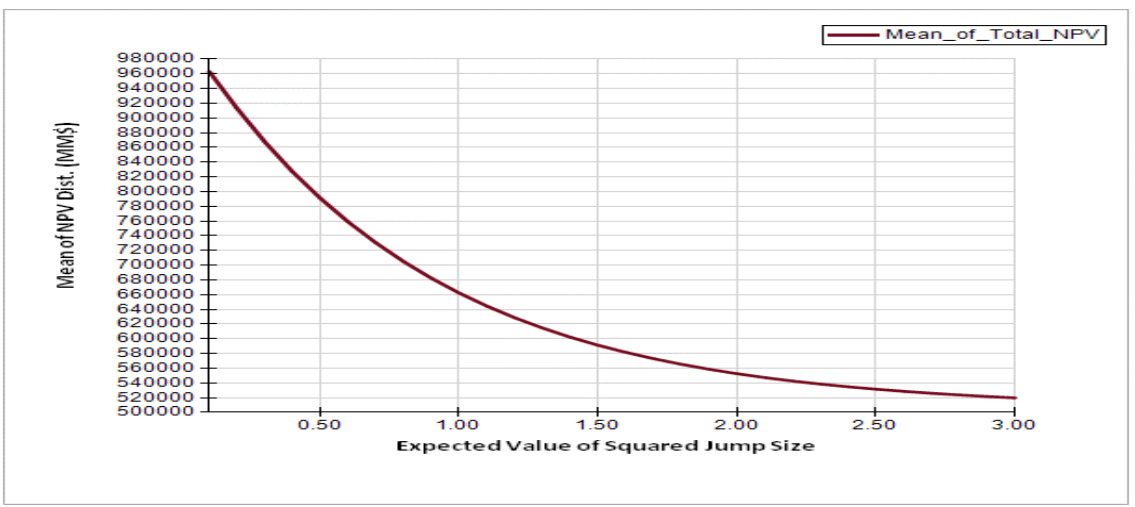

Figure 6.38: Sensitivity of Total NPV to the mean of squared jump size

\subsection{Discussion}

The results of the study indicate that sophisticated crude oil price forecasting models do not add value to the production allocation decision making problem. On the other hand, these sophisticated models significantly impact the value of a project and the NPV. This section discusses the major findings, challenges and limitations of the study.

Table 6.29 and Figure 6.38 show the optimum allocation decisions for the different price forecast models. The Flat Price, the ILD, the MRM, and the MRJM models have the same optimum allocation decision while the Floating Price and the GBM models have a slightly different allocation decision. However, the difference between the two optimum allocation decisions is negligible (77 MBD between Fields 8 \& 19); the 
impact of $77 \mathrm{MBD}$ on 8,000 MBD portfolio is negligible. A possible explanation for why the GBM and the Floating Price model have similar results is that the drift term in the GBM model acts like the rate of price increases in the Floating Price model. The different price forecasting models do not impact the optimum production allocation decision. As a result, there is no value in mitigating the uncertainty of which price forecasting model the oil market mimics, hence the VOI for this particular decision is zero.

Table 6.30 shows the total NPV, or the mean of total NPV and the standard deviation of total NPV for the different price forecasting models. The total NPV of the Floating Price model is close to the mean of the total NPV of the GBM model; both of these models incorporate rate of price increases or price drift. The other four price forecast models resulted in values close to that of the total NPV and the mean of total NPV. The standard deviation of the MRJM model is very large; this huge standard deviation resulted from the jumps effect. In other words, the jumps in MRJM dictate the volatility of the model and hence the standard deviation; consequently, the impact of the price volatility is minimal in the MRJM. The GBM model is a diffusion process of a lognormal distribution; this process resulted in the largest standard deviation among the price forecast models. As shown in Table 6.30, the price forecasting models significantly impact the value of the companies as represented by the total NPVs. The table shows that a wide range of NPVs (MM\$ 720,000-3,000,000) can be attained from different crude oil prices forecasting models. In other words, it is essential that oil companies identify the behavior of the oil market's price, during the early stages of the project planning process, to accurately calculate a company's wealth, which impacts future development projects.

Estimating field parameters was a challenge because revealing actual field data is sensitive. The fields parameters used in the study are reasonably estimated by industry 
experts. Another challenge was the estimating the jumps parameters for the MRJM price forecasting model. The jumps in the MRJM represent the arrival of unusual information; price jump parameters were very difficult to estimate because of the nature of the jumps (unpredictable). It is more logical to use reasonable jump parameters than estimate them from historical data, as suggested by Dias (2010a). Consequently, widely used jump parameters are used for our study.

The study focused on the impact of an individual modeling parameter on the optimum allocation decision. Project planning parameters such as fixed and variable OPEX, and depletion rates were not correlated to crude oil prices, which might impact the findings of the study. In practice, most of the project-planning parameters are a function of crude oil prices. These parameters were not correlated in the study to reduce the computational load on the integrated model. Correlating these parameters to crude oil prices may increase the reliability of the findings. This limitation should not change the findings of the study since the findings rely on a range of values (NPV distribution). In other words, correlating the parameters to crude oil price impacts the range of the NPV, but it should not change the shape of the total NPV distribution, hence it should not impact the optimum allocation decision. 
Table 6.29: Optimum Allocation Decision for Different Price Forecast Models

\begin{tabular}{|c|c|c|c|c|c|c|c|}
\hline Crude Grade & Field & Flat Price Model & $\begin{array}{c}\text { Floating Price } \\
\text { Model }\end{array}$ & ILD Model & GBM Model & MRM Model & MRJM Model \\
\hline \multirow{3}{*}{ 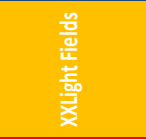 } & 1 & 153 & 153 & 153 & 153 & 153 & 153 \\
\hline & 2 & 329 & 329 & 329 & 329 & 329 & 329 \\
\hline & 3 & 205 & 205 & 205 & 205 & 205 & 205 \\
\hline \multirow{4}{*}{ 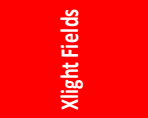 } & 4 & 822 & 822 & 822 & 822 & 822 & 822 \\
\hline & 5 & 548 & 548 & 548 & 548 & 548 & 548 \\
\hline & 6 & 274 & 274 & 274 & 274 & 274 & 274 \\
\hline & 7 & 377 & 377 & 377 & 377 & 377 & 377 \\
\hline \multirow{8}{*}{ 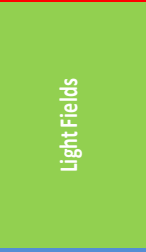 } & 8 & 33 & 110 & 33 & 110 & 33 & 33 \\
\hline & 9 & 247 & 247 & 247 & 247 & 247 & 247 \\
\hline & 10 & 342 & 342 & 342 & 342 & 342 & 342 \\
\hline & 11 & 548 & 548 & 548 & 548 & 548 & 548 \\
\hline & 12 & 822 & 822 & 822 & 822 & 822 & 822 \\
\hline & 13 & 438 & 438 & 438 & 438 & 438 & 438 \\
\hline & 14 & 438 & 438 & 438 & 438 & 438 & 438 \\
\hline & 15 & 411 & 411 & 411 & 411 & 411 & 411 \\
\hline \multirow{4}{*}{ 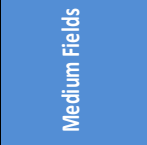 } & 16 & 123 & 123 & 123 & 123 & 123 & 123 \\
\hline & 17 & 308 & 308 & 308 & 308 & 308 & 308 \\
\hline & 18 & 247 & 247 & 247 & 247 & 247 & 247 \\
\hline & 19 & 545 & 468 & 545 & 468 & 545 & 545 \\
\hline \multirow{4}{*}{ 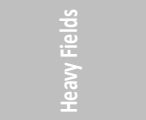 } & 20 & 205 & 205 & 205 & 205 & 205 & 205 \\
\hline & 21 & 222 & 222 & 222 & 222 & 222 & 222 \\
\hline & 22 & 197 & 197 & 197 & 197 & 197 & 197 \\
\hline & 23 & 164 & 164 & 164 & 164 & 164 & 164 \\
\hline
\end{tabular}

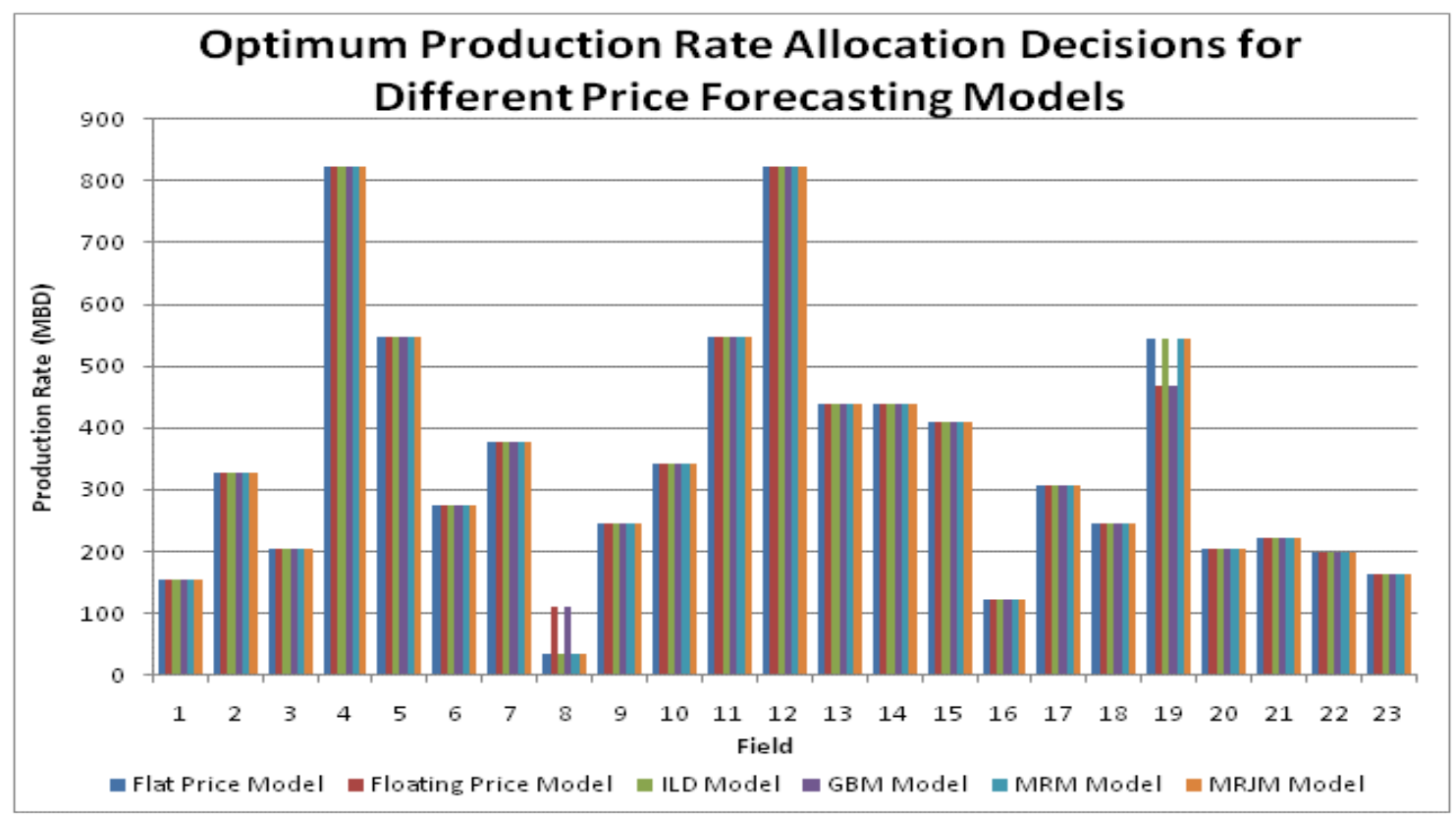

Figure 6.39: Optimum Production Allocation Decisions for Different Price Forecasting Models 
Table 6.30: Total NPV, Mean of the Total NPV, and Standard Deviation of Total NPV for Different Price Forecast Models

\begin{tabular}{|lrrrrrr|}
\hline Price Forecast Model & Flat Price & Floating Price & ILD & GBM & MRM & MRMJ \\
\cline { 2 - 7 } Total NPV (MM\$) & 720,306 & $3,154,895$ & & & & \\
E[Total NPV] (MM\$) & & & 809,072 & $3,272,396$ & 730,536 & 793,287 \\
SD[Total NPV] (MM\$) & & & 186,918 & $4,167,279$ & 94,248 & 598,866 \\
\hline
\end{tabular}




\section{CHAPTER 7: THE VALUE OF AN ACCURATE PRICE CORRELATION MODEL (VOI ANALYSIS)}

The significance of the correlation models assumption(s) on the production allocation decision is shown in the optimization sensitivity analysis conducted for the ILD price forecasting model where a small change in a price correlation factor can result in a dramatic change in the optimum allocation decision. Since the optimum allocation decision is responding to the changes in the price correlation factors (different factor values can have different allocation decisions), there should be a maximum monetary value (the willingness of an oil company to pay) to mitigate the uncertainty in the price correlation model. This maximum monetary value is the Value of Information (VOI). This chapter discusses the process of calculating the value of mitigating the risk involved in the price correlation model, thus increasing the accuracy of the correlation model.

Our study involves 5 different crude prices; the price of one crude type (Heavy crude) was forecasted and the remaining four crude prices were correlated to the forecasted price. In other words, there are 4 correlation factor values in the study. Here, the values of mitigating the risk involved in these correlation factors are estimated and analyzed. Note that this analysis assumes perfect knowledge of information, which means that the estimated value of the information is the maximum amount an oil company would invest to reduce the risk in that information.

\subsection{The VOI Analysis on Heavy-Medium Price Correlation Model}

Figure 7.1 describes the process of estimating the monetary value of an accurate Heavy-Medium price correlation model. To calculate the VOI of a more accurate price model, two cases (base case and alternate case) are considered as shown in Figure 7.1. The price correlation factors used in the VOI analysis are 1.01 and 1.05 for the base and 
the alternate cases, respectively. Using the integrated optimization model for the ILD price model, different optimum allocation decisions are assigned for each case as shown in Table 7.1. Put simply, the value of a more accurate price correlation model (VOI), the willingness of an oil company to pay, is the difference between the expected value of NPV (B) and the expected value of NPV (C) in Figure 7.1, which is 8,181 million dollars. The process begins by asking "Shall we obtain information on the price correlation factor prior to making decisions?" If the information is not obtained, the optimum production allocation for the base case is assigned. The base case optimum allocation decision is optimum if the correlation factor for the base case is realized (with E[NPV] $A$ of 809,749 million dollars). On the other hand, this allocation decision is sub-optimum if the correlation factor for the alternate case is realized (with E[NPV] $C$ of 805,219 million dollars).

The other branch of the decision tree represents obtaining the information of the correlation factor and informative decision making. As a result, the production allocation decision is optimum for both cases since the decisions are made with perfect knowledge of the actual correlation factors. The $\mathrm{E}[\mathrm{NPV}] B$, for the alternate case, is 813,400 million dollars. The VOI is the difference between the expected value of the NPVs resulting from the alternate case decision based on alternate case correlation factors and the alternate case decision given the base case correlation factors (E[NPV] B - E[NPV] C). The VOI is 8,181 million dollars. Acquiring the calculated value of perfect information depends on the cost of the acquisition of that information. In other words, acquiring the perfect information is warranted if the cost of acquiring the information is less than the value of the information calculated above.

Figure 7.2 shows the value of acquiring an accurate price correlation model for Heavy-Medium as a function of the correlation factor of Heavy-Medium crudes. The 
VOI is calculated for various alternate cases. In other words, the VOI is calculated as the correlation factor is varied between 0.05 and 3. Note that as the Heavy-Medium correlation factor increases, the value of the Medium crude price increases and viceversa. From the figure, as the price correlation factor increases more than the value of the base case (1.01), the VOI rapidly increases. On the other hand, the VOI increases in relatively small increments, as the price correlation factor decreases below the value of the base case. This curve reflects the optimum production allocation decision of the base case, which assigns most of the Medium crude fields at minimum production rates. As a result, as the Heavy-Medium correlation factor increases above the value of the base case (the value of the Medium crude price increases), the optimizer allocates more production rates to the Medium crude fields. This big deviation from the base case allocation decision increases the VOI. On the other hand, as the price correlation factor decreases (the value of the Medium crude price decreases), the optimum allocation decision is very similar to the one of the base case (most of the Medium fields are at minimum rates); the only difference is $299 \mathrm{MBD}$, which is re-allocated from Field 19 (Medium crude). Consequently, there is not a significant change from the base case, thus the VOI increases at relatively smaller rate. 


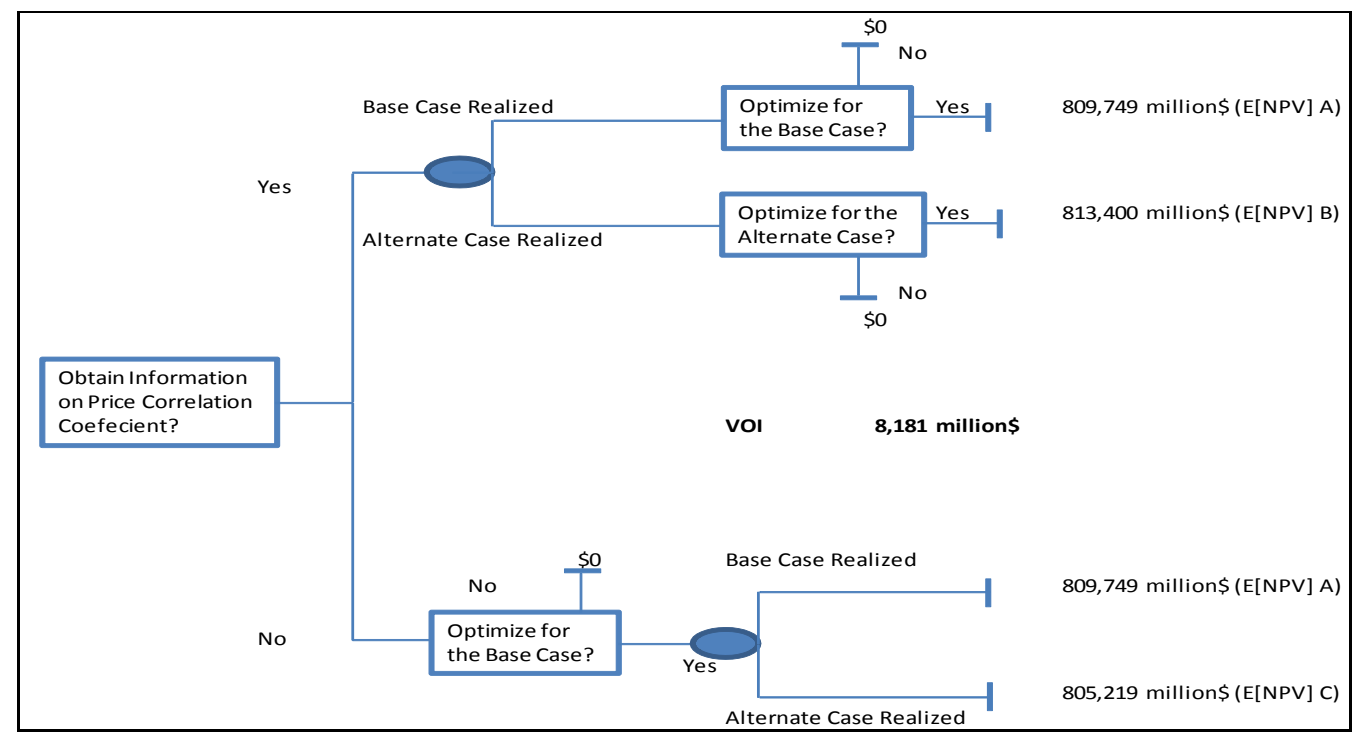

Figure 7.1: Decision Tree to Evaluate the Value of Mitigating the Uncertainty in the Heavy-Medium Price Correlation Factor

Table 7.1: The Optimum Production Allocation Decisions for the Base and Alternate Cases of Heavy-Medium Price Correlation Factor

\begin{tabular}{|ccc|}
\hline Field & $\begin{array}{c}\text { Base Case Optimum } \\
\text { Production Rate } \\
\text { (MBD) }\end{array}$ & $\begin{array}{c}\text { Alternate Case } \\
\text { Optimum Production } \\
\text { Rate (MBD) }\end{array}$ \\
\hline \hline 1 & 153 & 153 \\
2 & 329 & 329 \\
3 & 205 & 205 \\
4 & 822 & 822 \\
5 & 548 & 548 \\
6 & 274 & 274 \\
7 & 377 & 377 \\
8 & 33 & 33 \\
9 & 247 & 74 \\
10 & 342 & 103 \\
11 & 548 & 548 \\
12 & 822 & 822 \\
13 & 438 & 132 \\
14 & 438 & 438 \\
15 & 411 & 123 \\
16 & 123 & 123 \\
17 & 308 & 463 \\
18 & 247 & 822 \\
19 & 545 & 822 \\
\hline 20 & 205 & 205 \\
21 & 222 & 222 \\
22 & 197 & 197 \\
23 & 164 & 164 \\
\hline & & \\
\hline
\end{tabular}




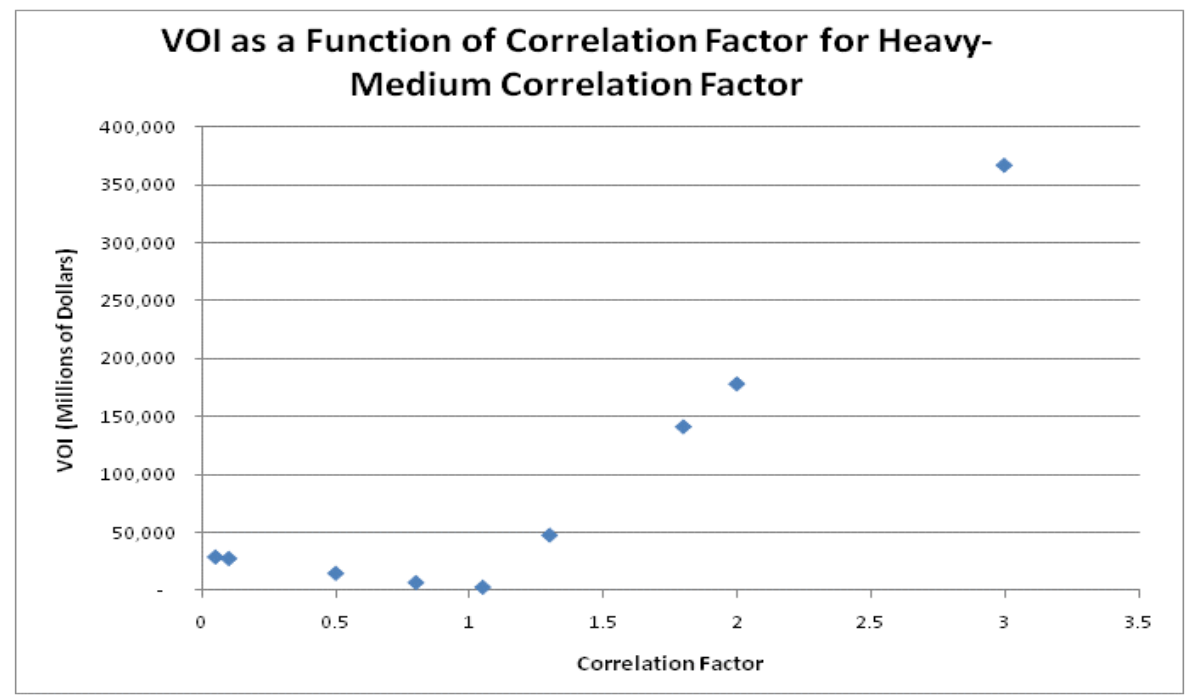

Figure 7.2: Value of an Accurate Heavy-Medium Correlation Model as a Function of Correlation Factor

\subsection{The VOI Analysis on Heavy-Light Price Correlation Model}

The process of estimating the value of a more accurate Heavy-Light price correlation model is described in Figure 7.3. The price correlation factors used in the VOI analysis are 1.03 and 0.95 for the base and the alternate cases, respectively. Table 7.2 shows the optimum allocation decisions for the base and alternate cases, using the ILD price model. The value of a more accurate price correlation model (VOI) is the difference between the expected value of NPV (B) and the expected value of NPV (C) in Figure 7.3, which is 8,328 million dollars. Acquiring the perfect information depends on the cost of the acquisition of that information. In other words, acquiring the perfect information is warranted if the cost of acquiring the information is less than the value of the information calculated above.

Figure 7.4 shows the value of acquiring an accurate price correlation model for Heavy-Light as a function of the correlation factor of Heavy-Light crudes. The figure shows the VOI as the price correlation factor for the alternate case is varied between 0.05 
and 5. Note that as the Heavy-Light correlation factor increases, the value of the Light crude price increases. From the figure, as the price correlation factor decreases below the value of the base case (1.03), the VOI rapidly increases. On the other hand, the VOI increases in relatively small increments, as the price correlation factor increases above the value of the base case. This curve resulted from the optimum allocation decision of the base case. In the base case, the optimizer assigns most of the Light fields at maximum production rates. As a result, as the Heavy-Light correlation factor decreases below the value of the base case (the value of the Light crude price decreases), the optimizer reduces the production rates allocated to the Light fields and increase the rates from the Medium and Heavy crude fields. This relatively large deviation from the base case decision increases the VOI. On the other hand, as the price correlation factor increases (the value of the Light crude price increases), the optimizer allocates more production rates to the Light crude fields. However, most of the Light fields are producing at maximum production rate, thus the VOI increases at relatively smaller rate.

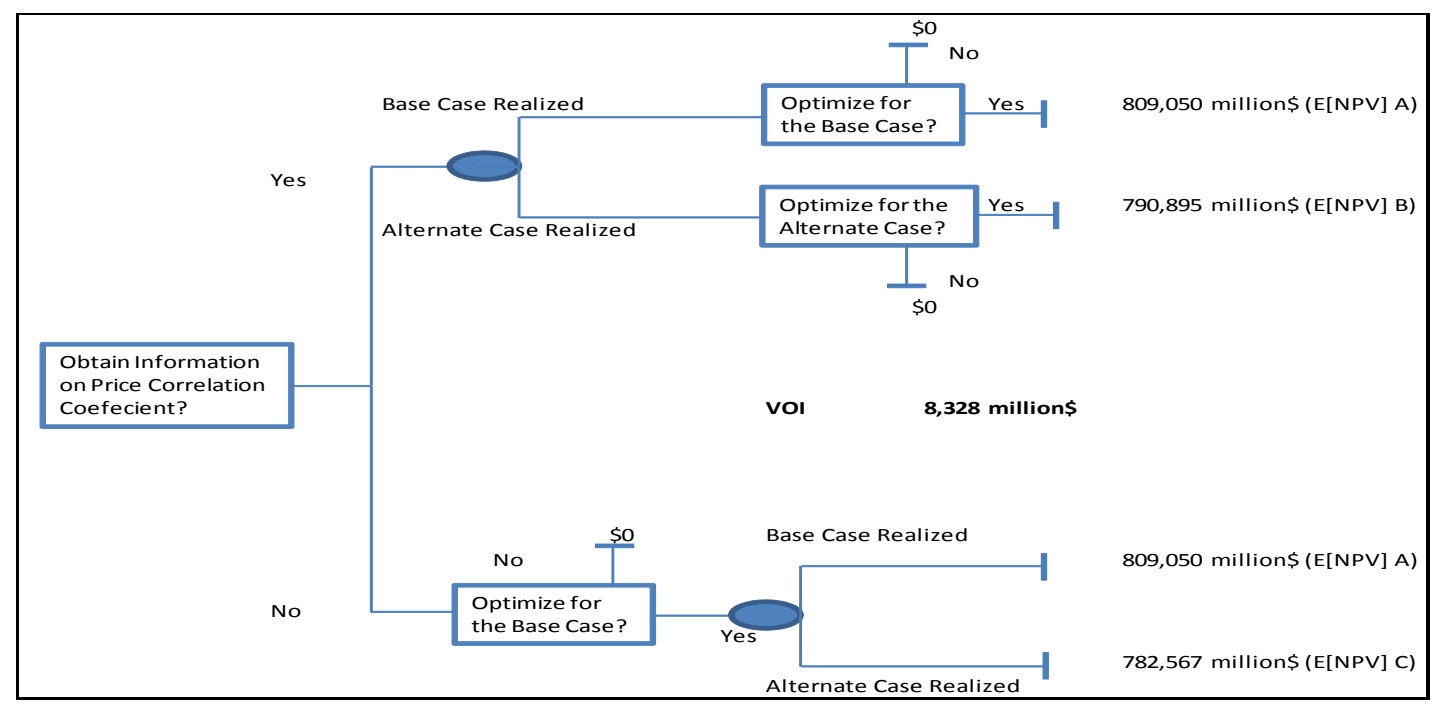

Figure 7.3: Decision Tree to Evaluate the Value of Mitigating the Uncertainty in the Heavy-Light Price Correlation Factor 
Table 7.2: The Optimum Production Allocation Decisions for the Base and Alternate Cases of Heavy-Light Price Correlation Factor

\begin{tabular}{|ccc|}
\hline Field & $\begin{array}{c}\text { Base Case Optimum } \\
\text { Production Rate } \\
\text { (MBD) }\end{array}$ & $\begin{array}{c}\text { Alternate Case } \\
\text { Optimum Production } \\
\text { Rate (MBD) }\end{array}$ \\
\hline \hline 1 & 153 & 153 \\
2 & 329 & 329 \\
3 & 205 & 205 \\
4 & 822 & 822 \\
5 & 548 & 548 \\
6 & 274 & 274 \\
7 & 377 & 377 \\
8 & 33 & 33 \\
9 & 247 & 74 \\
10 & 342 & 103 \\
11 & 548 & 164 \\
12 & 822 & 247 \\
13 & 438 & 132 \\
14 & 438 & 132 \\
15 & 411 & 123 \\
16 & 123 & 411 \\
17 & 308 & 1027 \\
18 & 247 & 822 \\
19 & 545 & 822 \\
\hline 20 & 205 & 619 \\
21 & 222 & 222 \\
22 & 197 & 197 \\
23 & 164 & 164 \\
\hline & & \\
\hline
\end{tabular}

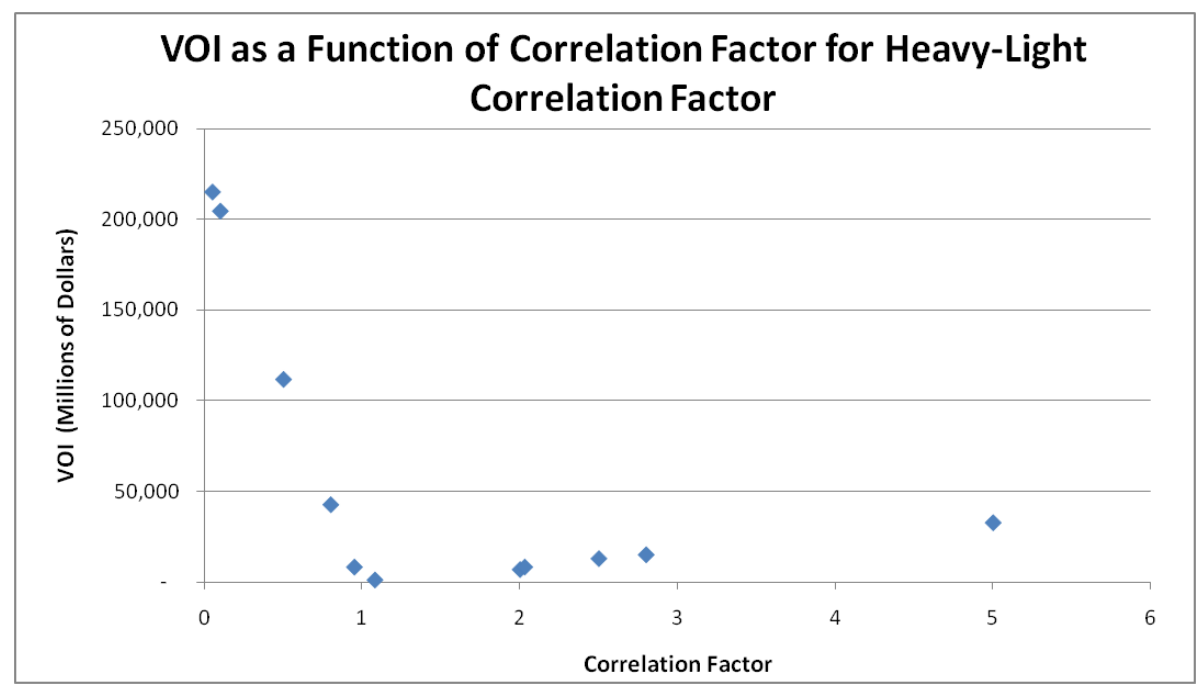

Figure 7.4: Value of an Accurate Heavy-Light Correlation Model as a Function of Correlation Factor 


\subsection{The VOI Analysis on Heavy-XLight Price Correlation Model}

Figure 7.5 describes the process of estimating the monetary value of an accurate Heavy-XLight price correlation model. The price correlation factors used in the VOI analysis are 1.05 and 0.80 for the base and the alternate cases, respectively. Table 7.3 shows the optimum allocation decision for the base and alternate correlation factors. The value of mitigating the risk in the price correlation model for Heavy-XLight (VOI) is 27,075 million dollars as shown in Figure $7.5(\mathrm{E}[\mathrm{NPV}(\mathrm{B})]-\mathrm{E}[\mathrm{NPV}(\mathrm{C})])$. This VOI means that acquiring the information to mitigate the risk is warranted if the VOI is greater than the cost of acquisition.

Figure 7.6 shows the value of acquiring an accurate price correlation model for Heavy-XLight as a function of the correlation factor of the Heavy-XLight crudes. The figure shows the VOI as the price correlation factor for the alternate case is varied between 0.10 and 2. From the figure, as the price correlation factor decreases (decreasing the value of XLight crude price) below the value of the base case (1.05), the VOI rapidly increases. On the other hand, the VOI is zero, as the price correlation factor increases above the value of the base case. The base case allocation decision assigns all the XLight fields at maximum rates. As a result, there is no value to mitigate the risk if the correlation factor of the alternate case is above the factor of the base case because the optimum decision will not change (All XLight field at maximum rates). However, the difference of the optimum allocation decision between the base and alternate cases rapidly increases as the price correlation factor decreases, increasing the VOI. 


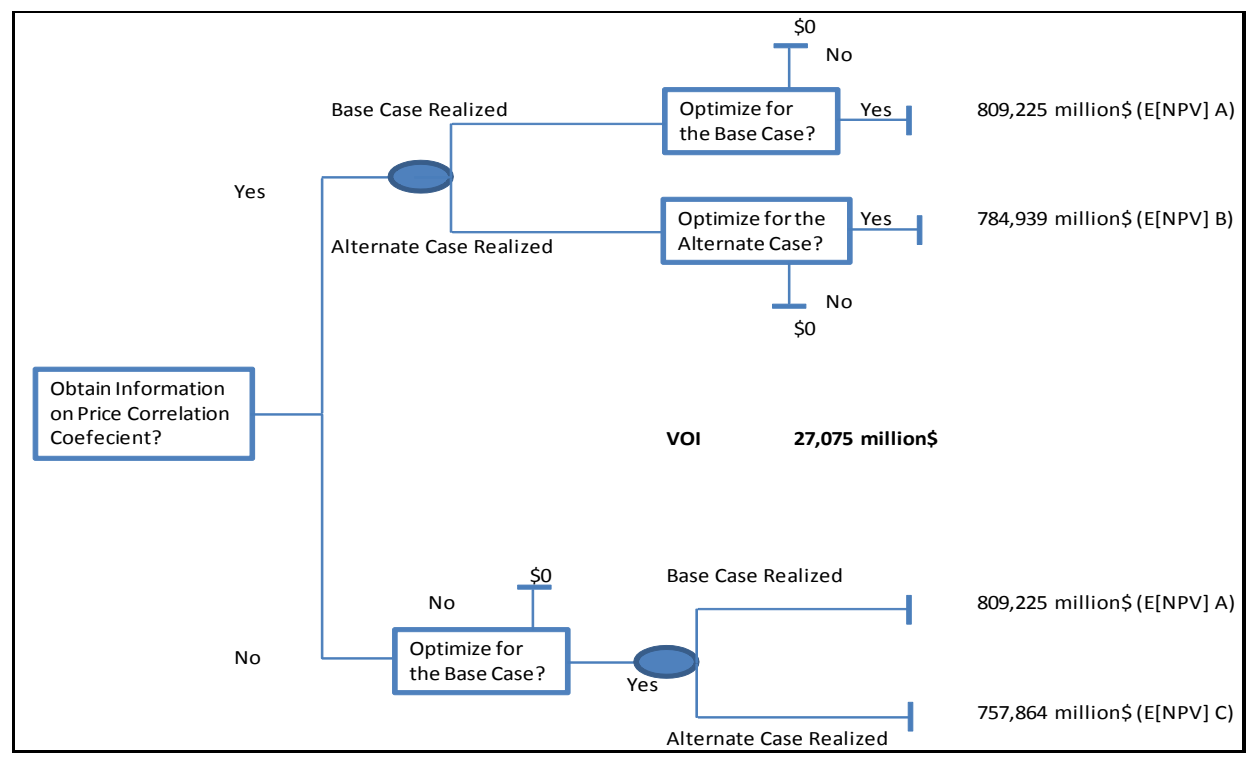

Figure 7.5: Decision Tree to Evaluate the Value of Mitigating the Uncertainty in the Heavy-XLight Price Correlation Factor

Table 7.3: The Optimum Production Allocation Decisions for the Base and Alternate Cases of Heavy-XLight Price Correlation Factor

\begin{tabular}{|c|c|c|}
\hline Field & $\begin{array}{c}\text { Base Case } \\
\text { Optimum } \\
\text { Production Rate } \\
\text { (MBD) }\end{array}$ & $\begin{array}{c}\text { Alternate Case } \\
\text { Optimum } \\
\text { Production Rate } \\
\text { (MBD) }\end{array}$ \\
\hline 1 & 153 & 153 \\
\hline 2 & 329 & 329 \\
\hline 3 & 205 & 205 \\
\hline 4 & 822 & 247 \\
\hline 5 & 548 & 164 \\
\hline 6 & 274 & 82 \\
\hline 7 & 377 & 113 \\
\hline 8 & 33 & 33 \\
\hline 9 & 247 & 247 \\
\hline 10 & 342 & 342 \\
\hline 11 & 548 & 548 \\
\hline 12 & 822 & 822 \\
\hline 13 & 438 & 438 \\
\hline 14 & 438 & 438 \\
\hline 15 & 411 & 411 \\
\hline 16 & 123 & 123 \\
\hline 17 & 308 & 871 \\
\hline 18 & 247 & 822 \\
\hline 19 & 545 & 822 \\
\hline 20 & 205 & 205 \\
\hline 21 & 222 & 222 \\
\hline 22 & 197 & 197 \\
\hline 23 & 164 & 164 \\
\hline
\end{tabular}




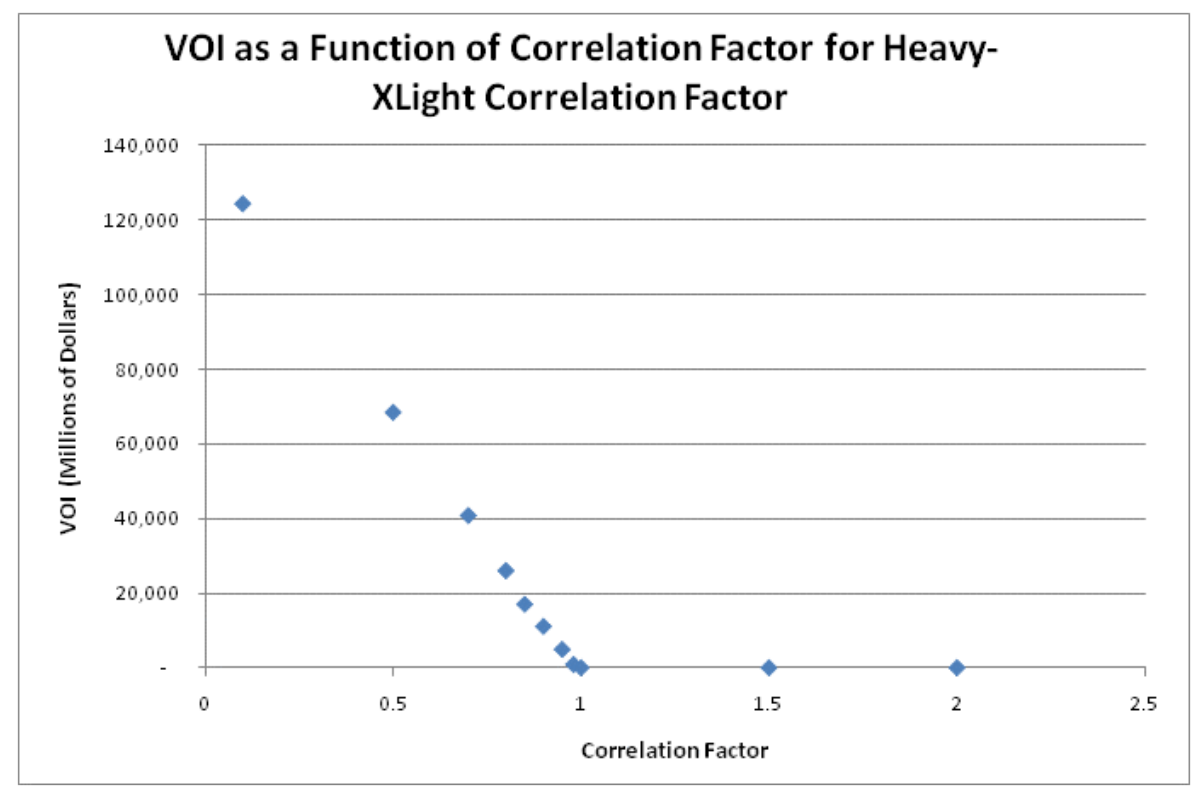

Figure 7.6: Value of an Accurate Heavy-XLight Correlation Model as a Function of Correlation Factor

\subsection{The VOI Analysis on Heavy-XXLight Price Correlation Model}

Figure 7.7 describes the process of estimating the value of obtaining the perfect information on correlation factor of the Heavy-XXLight price correlation model. The price correlation factors used in the VOI analysis are 1.08 and 0.50 for the base and the alternate cases, respectively. The optimum allocation decisions for the two cases are shown in Table 7.4. The value of a more accurate price correlation model (VOI) is 22,851 million dollars $(\mathrm{E}[\mathrm{NPV}(\mathrm{B})-\mathrm{E}[\mathrm{NPV}(\mathrm{C})])$. The decision on obtaining the perfect information depends on the VOI and the cost of obtaining the uncertain information.

The value of obtaining an accurate price correlation model for Heavy-XXLight crude prices are plotted as a function of the correlation factor of the alternate case as shown in Figure 7.8. In other words, the VOI is calculated as the correlation factor of the alternate case is varied between 0.10 and 1.50 . The figure shows that the VOI increases as the correlation factor decreases below the value of the base case (1.08). On the other 
hand, the VOI is zero as the price correlation factor increases above the value of the base case. In the base case optimum allocation decision, the XXLight fields are producing at maximum rates. As a result, there is no value in acquiring more information on the price correlation model if the alternate case is greater than the value of the base case. The reason of the zero VOI is that the optimum allocation decision does not change as the correlation factor of the alternate case increases (XXLight fields are producing at maximum rates). However, the VOI increases because the optimum allocation decision the deviation of the optimum allocation decision, as the correlation factor of the alternate case decreases below the value of the base cases, increases the VOI. the difference of the optimum allocation decision between the base and alternate cases rapidly increases as the price correlation factor decreases, increasing the VOI. However, the VOI increases as the correlation factor decreases below the value of the base case because the difference of the optimum allocation decision between the base and alternate cases increases as the price correlation factor decreases.

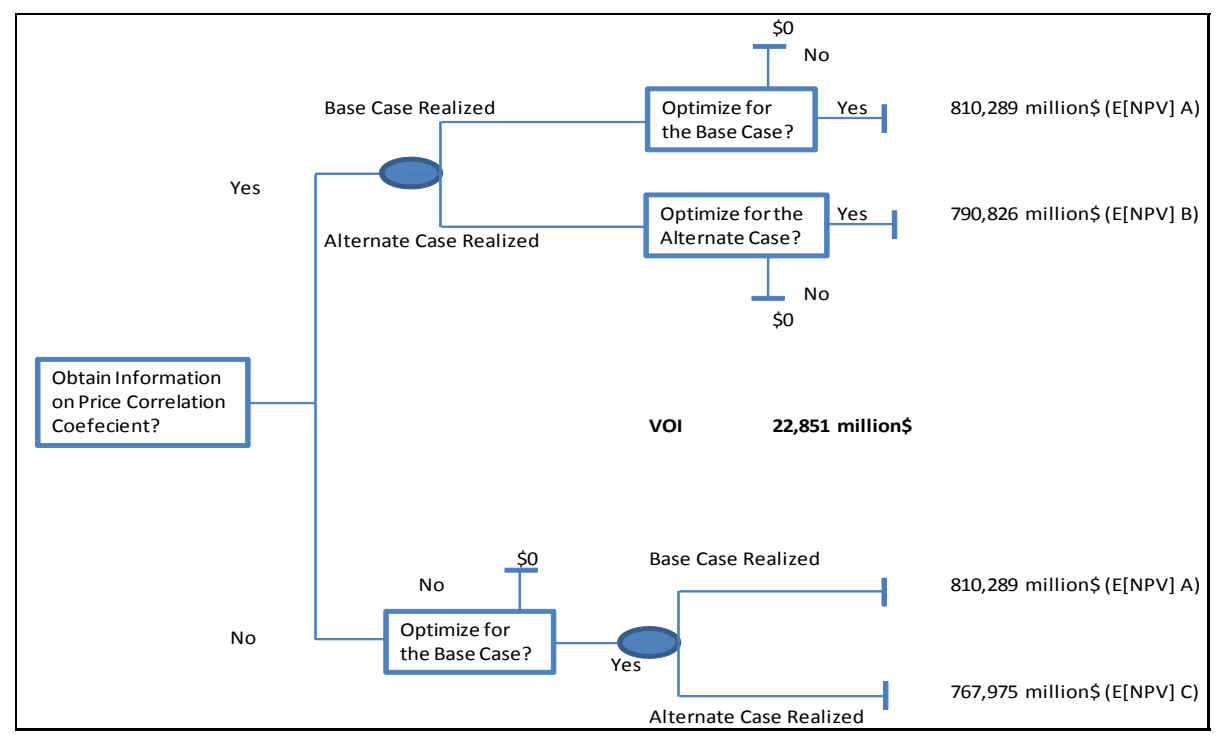

Figure 7.7: Decision Tree to Evaluate the Value of Mitigating the Uncertainty in the Heavy-XXLight Price Correlation Factor 
Table 7.4: The Optimum Production Allocation Decisions for the Base and Alternate Cases of Heavy-XXLight Price Correlation Factor

\begin{tabular}{|ccc|}
\hline Field & $\begin{array}{c}\text { Base Case Optimum } \\
\text { Production Rate } \\
\text { (MBD) }\end{array}$ & $\begin{array}{c}\text { Alternate Case } \\
\text { Optimum Production } \\
\text { Rate (MBD) }\end{array}$ \\
\hline \hline 1 & 153 & 46 \\
2 & 329 & 99 \\
3 & 205 & 62 \\
\hline 4 & 822 & 822 \\
5 & 548 & 548 \\
6 & 274 & 274 \\
7 & 377 & 377 \\
8 & 33 & 33 \\
9 & 247 & 247 \\
10 & 342 & 342 \\
11 & 548 & 548 \\
12 & 822 & 822 \\
13 & 438 & 438 \\
14 & 438 & 438 \\
15 & 411 & 411 \\
16 & 123 & 123 \\
17 & 308 & 308 \\
18 & 247 & 451 \\
19 & 545 & 822 \\
\hline 20 & 205 & 205 \\
21 & 222 & 222 \\
22 & 197 & 197 \\
23 & 164 & 164 \\
\hline
\end{tabular}

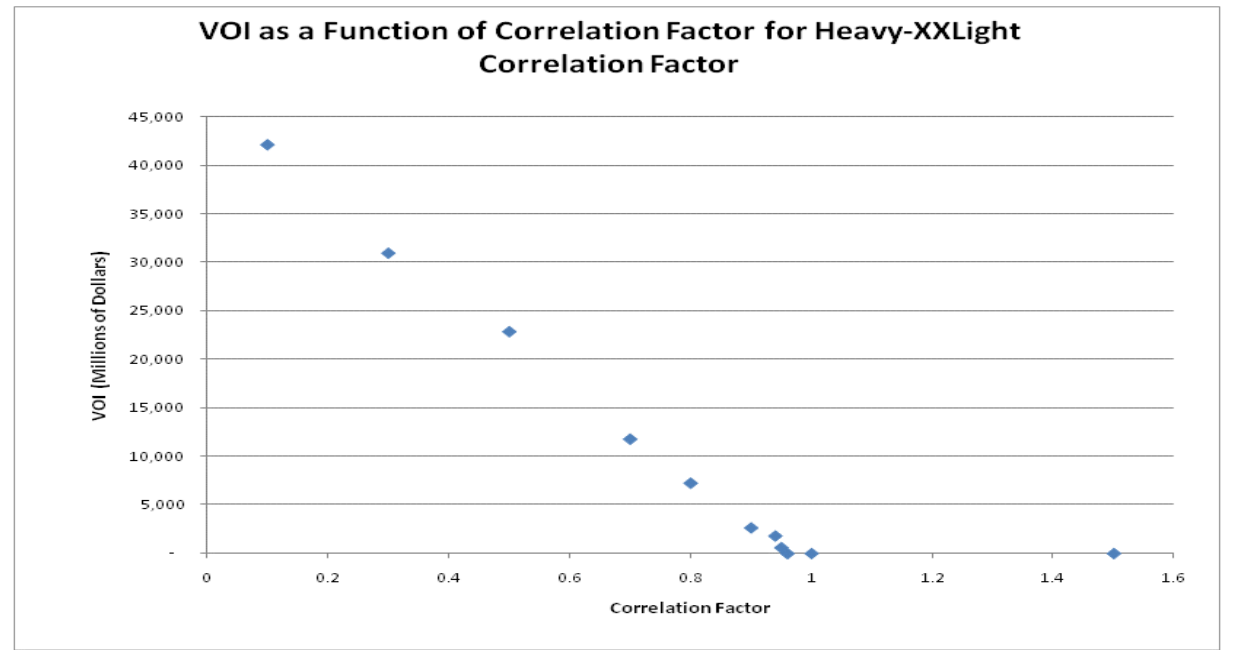

Figure 7.8: Value of an Accurate Heavy-XXLight Correlation Model as a Function of Correlation Factor 


\section{CHAPTER 8: CONCLUSIONS AND RECOMMENDATIONS FOR FUTURE WORK}

Allocating production volumes across a portfolio of producing assets is a complex optimization problem that is faced by operators on a daily basis; crude oil price uncertainty adds to that complexity. In this thesis, we proposed an uncertainty analysis and an integrated optimization model to solve this optimization problem. The proposed workflow and the integrated model accounted for various technical attributes, facility constraints, and project costs for each producing asset. Moreover, the company's target total rate was honored in the integrated model. The outcomes of the optimization problem were compared for various price models. To mitigate the crude oil price uncertainty and estimate the value of implementing a more accurate crude oil price model, Value of Information (VOI) analysis was performed.

\subsection{Concluding Remarks}

1. The type of crude price forecast model, used in the optimization model, does not impact the optimum production allocation decision. On the other hand, the type of price model significantly affects the value of the project (NPV). As a result, the type of price model is a key parameter in the development project planning stage because the price model significantly impacts the value of a development project.

2. The assumptions and parameters of the price correlation model have a significant impact on the allocation decisions. The impacts of these assumptions and parameters are analyzed; moreover, the value of a more accurate price correlation model was estimated. The assumptions of the 
correlation model define the price differential of different crudes; these price differentials play a major role in the production allocation decision.

3. Various price forecasting models were integrated with economic, optimization, and simulation models to evaluate the impact of the price models (model type and model assumptions) on the production allocation decisions.

4. The accuracy and reliability of the study depend on data availability, and time computational limitations.

5. The simple integrated model provided a reliable solution for the allocation decision problem.

6. The integrated optimization model requires a large amount of computations. This computational burden can dramatically increase when incorporating a more reliable integrated model. As a result, two modeling options were not considered in the study: correlating variable OPEX and crude oil prices, and allowing the fields to shutdown in the integrated model. It may not be practical to incorporate these two options into the integrated model although they might increase the reliability of the outcomes.

7. Risk Solver Platform (RSP), a fast solving algorithm, eliminated practical constraints allowing for a robust sensitivity analysis. In other words, the speed of the RSP allowed for a large number of different scenarios of modeling parameters to be analyzed.

8. Anticipatory error (error of perfect information) was avoided in the study by assigning a single production rate value for each field throughout the life of the project. As a result, the optimizer could not respond to the 163 
revealed uncertain variables (crude oil prices). This error is common in optimization under uncertainty studies.

9. Our integrated model can be used to determine the optimum allocation decision on a daily basis. In other words, the integrated model can solve the problem for one time period; consequently, this model is a handy tool that enables management to modify production allocation decisions.

\subsection{Recommendations for Further Research}

\subsubsection{Recommendations Related to Price Correlation}

1. The impact of the assumptions and parameters of the price correlation models was analyzed in the study. A potential area for future research is the parameters and the assumptions of the price correlation models for the different crudes. A dedicated study on these assumptions and parameters should considerably impact the production allocation decisions.

2. In our integrated model, economic evaluation parameters (e.g., OPEX variable costs, depletion rates, etc.) were not correlated to the crude oil prices. In other words, these evaluation parameters do not respond to any change in the crude oil prices. One may extend the study by correlating the economic evaluation parameters to crude oil prices. Historically, crude oil prices and various economic evaluation parameters are strongly correlated, which might impact the total NPV of the projects and the optimum allocation decision.

3. In our study, regression analysis was used to correlate the prices of different crudes. This approach may be extended to correlate the crude oil prices using the probability distribution of the spread in these prices. 


\subsubsection{Recommendations Related to Optimization Process}

1. Field production rates were the decision variables for the optimization problem in our study. Decision variables can be extended to include several parameters besides the field production rates. An interesting decision variable for major producers is depletion rate (rate at which reservoirs are depleted). The importance of depletion rates is related to the time value of money; due to the discounting process, a dollar today is worth more than a dollar tomorrow. As a result, the obvious answer to a depletion rate problem is to deplete the reservoir at the maximum producible rate. However, the depletion rate problem is a more complicated constrained problem; the constraints include the health and integrity of a reservoir, the safety and economy of operations, demand, politics, and management needs.

2. Total NPV was used in this study as the objective function; Total NPV is the commonly used objective function (performance attribute) in the oil industry. Functionality requirement (objective function) is a potential parameter for further investigation. Objective function can be modified to serve management's needs. For example, daily cost can be a proper objective function for a project manager who is interested in daily profit rather than the long-term value of the project. Using the daily cost as the objective function for the allocation optimization problem should significantly impact the optimum allocation decision because the profits and revenues are not involved in the optimization process. 
3. In our study, the total production target rate was a fixed value specified by management. The study can be extended to account for uncertainty in oil demand by modeling the total target rate as a probability distribution.

\subsubsection{Recommendations Related to Technical Process}

In the integrated model, it was assumed that the reservoir pressure and production rates are maintained throughout the life of the project. This approach can be extended to account for reservoir pressure decline by incorporating a reservoir simulator or a simple tank model into the integrated model. Moreover, incorporating a reservoir simulator into the integrated model can facilitate the investigation of the impact of the uncertainty in the reservoir parameters on the optimum allocation decision. 


\section{REFERENCES}

Campbell, J.M.Jr., Campbell, J.M.Sr., and Campbell, R.A.: "Analyzing and Managing Risky Investments”, John M. Campbell, Norman, OK (2001).

Dias, M. 2010a. Mean Reversion with Jumps Models, www.puc-rio.br/marco.ind/revjump.html. Downloaded 10 June 2010.

Dias, M. 2010b. Stochastic Process with Focus in Petroleum Applications, http://www.puc-rio.br/marco.ind/stochast.html. Downloaded 10 June 2010.

Dias, M. 2010c. Mean Reversion Models, http://www.puc-rio.br/marco.ind/revers.html.

Dias, M. 2010d. Monte Carlo Simulation of Stochastic Processes, http://www.pucrio.br/marco.ind/sim_stoc_proc.html.

Dixit, A. K. 1993. The Art of Smooth Pasting, Chap 1, 7 Bedford: The Gordon and Breach Publishing Group.

Dixit, A. K. and Pindyck, R. S. 1994. Investment under Uncertainty, Chap.3, 76-80 New Jersey: Princeton University Press.

Dyer, J. "Modeling the Underlying Asset: Stochastic Process." McCombs School of Business at the University of Texas at Austin, Lecture. 19 January 2011.

Energy Information Administration (EIA), http://www.eia.doe.gov/dnav/pet/pet pri_wco_k w.htm. Downloaded 1 January 2011.

Ettehad, A. 2009. Gas Storage Facility Design Under Uncertainty. MS Thesis, The University of Texas at Austin, Austin Texas (May 2009).

Ettehad, A., Jablonowski, C., Lake, L. 2009. Gas Storage Facility Design Under Uncertainty. Paper SPE 123987 presented at the SPE Annual Technical Conference and Exhibition, New Orleans, Louisiana, USA, 4-9 October 2009.

Faya, L. 2006. Real Asset Risk Management: an Integral Approach to Management Uncertainty in the E\&P Industry. MS Thesis, The University of Texas at Austin, Austin Texas (May 2006).

Faya, L.C., Lasdon, L., Lake, L.W., Dyer, J.S., and Chen: "Constructing Oil Exploration and Development Project Portfolios using Several Risk Measures-A Realistic Example", Paper SPE 107708 presented at the 2007 SPE Hydrocarbon Economics and Evaluation Symposium, Dallas, TX, 1-3 April 2007.

Hahn, W.J., and Dyer, J.S.: "Incorporating Mean-Reverting Price Forecasts into Exploration and Production Project Valuation", Paper SPE 94577 presented at the 2005 SPE Hydrocarbon Economics and Evaluation Symposium, Dallas, TX, 3-5 April 2005. 
Hartman, J. C. 2007. Engineering Economy and the Decision-Making Process, Chap 2, 39 New Jersey: Pearson Education, Inc.

Hosgor, E. 2009. Assessing the Impact of Commodity Price Assumptions on Facility Optimization: A case in Offshore Oil and Gas. MS thesis, The University of Texas at Austin, Austin, Texas (May 2009).

Jablonowski, C. "Time Value of Money \& Discounting." Crockell School of Engineering at the University of Texas at Austin, Lcture. 2 September 2009.

Jablonowski, C., Wiboonkij-Arphakul, C., and Neuhold, M. 2008. Estimating the Cost of Errors in Estimates Used During Concept Selection. SPE Proj Fac \& Const 3 (1): 1-6. SPE-110191-PA. doi: 10.2118/110191-PA.

MacAskie, R. 2007. The Value of Commodity Price Forecasts in the Presence of Futures. MS Thesis, The University of Texas at Austin, Austin Texas (August 2007).

Metcalf, G., and Hassett, K. 1995. "Investment under alternative return assumptions: comparing random walks and mean reversion," Journal of Economic Dynamics and control, Vol. 19, pp. 1741-88.

Olsen, G.T., Fariyibi, F.L., Lee, W.J., and McVay, D.A.: "Price Uncertainty Quantification Models Advance Project Economic Evaluations", Paper SPE 94610 presented at the 2005 SPE Hydrocarbon Economics and Evalutation Symposium, Dallas, TX, 3-5 April 2005.

Powell, S. G. and Baker, K. R. 2010. Management Science: The Art of Modeling with Spreadsheets, Chap. 16, 433 New Jersey: John Wiley \& Sons Inc.

Purwar, S. 2008. A Study on the Use of Proxy Reservoir Models in Field Development Optimization and Value of Information Problems. MS Thesis, The University of Texas at Austin, Austin, Texas (August 2008).

Staber, S.: "Futures-Based Modeling of the Oil Price for Realistic E\&P-Project Valuation", Paper SPE 104082 presented at the 2006 SPE International Oil \& Gas Conference and Exhibition, Beijing, China, 5-7 December 2006.

$\mathrm{Xu}$, L. 2010. Application of Real Options to Valuation and Decision Making in the Petroleum E\&P Industry. PhD Dissertation, The University of Texas at Austin, Austin Texas (December 2010). 


\section{VITA}

Abdulwahab Abdullatif Bukhari was born in Jeddah, Saudi Arabia. After completing his high school studies in 2000, he was granted a scholarship from Saudi ARAMCO (Saudi national oil company) to pursue his Bachelor of Science degree in

petroleum engineering at Louisiana State University (LSU). After his graduation on August 2005, he worked for Saudi ARAMCO for four years as planning analyst, production engineer, reservoir engineer, and reservoir simulation engineer. On September 2009, he joined the petroleum and Geosystems Engineering at The University of Texas at Austin to pursue his master's degree. Upon completion of master's degree in August 2011, he will join the graduate school of Penn State University to pursue his PhD degree in the Energy and Mineral Engineering department under the specialty of Petroleum Economics, and Risk and Decision Analysis.

Email address: bukharia@gmail.com

This thesis was typed by the author. 